\title{
ipcc
}

INTERGOVERnMENTAL PANEL ON climate chanৎe

\section{CLIMATE CHANGE 2014}

\section{Synthesis Report}

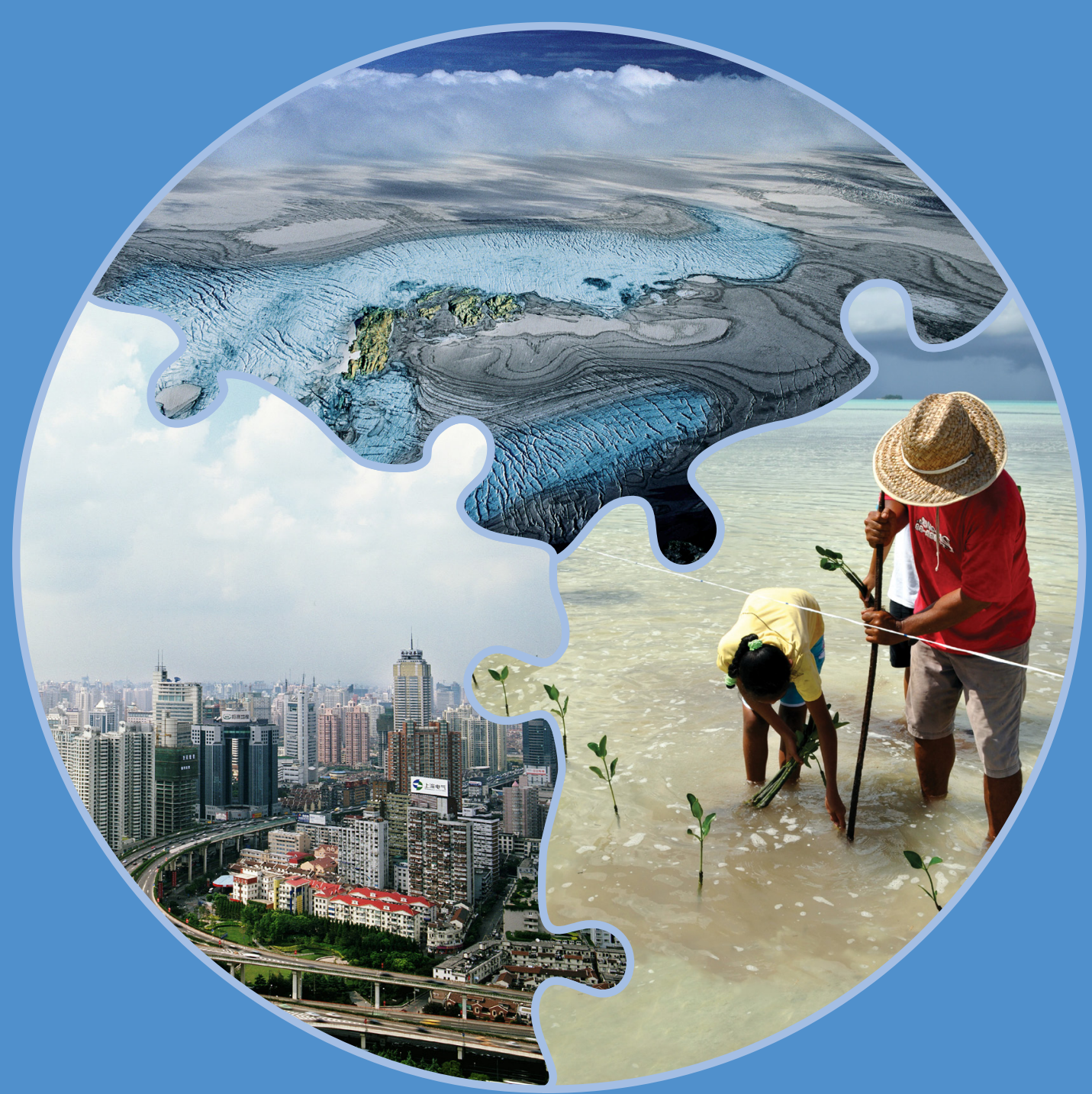

A REPORT OF THE

INTERGOVERNMENTAL PANEL ON CLIMATE CHANGE

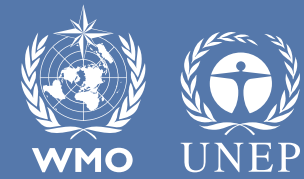




\section{Climate Change 2014 Synthesis Report}

\section{The Core Writing Team \\ Synthesis Report \\ IPCC}

\author{
Edited by \\ Rajendra K. Pachauri \\ Chairman \\ IPCC
}

\author{
Leo Meyer \\ Head, Technical Support Unit \\ IPCC
}

\section{Core Writing Team}

Rajendra K. Pachauri (Chair), Myles R. Allen (United Kingdom), Vicente R. Barros (Argentina), John Broome (United Kingdom), Wolfgang Cramer (Germany/France), Renate Christ (Austria/WMO), John A. Church (Australia), Leon Clarke (USA), Qin Dahe (China), Purnamita Dasgupta (India), Navroz K. Dubash (India), Ottmar Edenhofer (Germany), Ismail Elgizouli (Sudan), Christopher B. Field (USA), Piers Forster (United Kingdom), Pierre Friedlingstein (United Kingdom/Belgium), Jan Fuglestvedt (Norway), Luis Gomez-Echeverri (Colombia), Stephane Hallegatte (France/World Bank), Gabriele Hegerl (United Kingdom/Germany), Mark Howden (Australia), Kejun Jiang (China), Blanca Jimenez Cisneros (Mexico/UNESCO), Vladimir Kattsov (Russian Federation), Hoesung Lee (Republic of Korea), Katharine J. Mach (USA), Jochem Marotzke (Germany), Michael D. Mastrandrea (USA), Leo Meyer (The Netherlands), Jan Minx (Germany), Yacob Mulugetta (Ethiopia), Karen O'Brien (Norway), Michael Oppenheimer (USA), Joy J. Pereira (Malaysia), Ramón Pichs-Madruga (Cuba), Gian-Kasper Plattner (Switzerland), Hans-Otto Pörtner (Germany), Scott B. Power (Australia), Benjamin Preston (USA), N.H. Ravindranath (India), Andy Reisinger (New Zealand), Keywan Riahi (Austria), Matilde Rusticucci (Argentina), Robert Scholes (South Africa), Kristin Seyboth (USA), Youba Sokona (Mali), Robert Stavins (USA), Thomas F. Stocker (Switzerland), Petra Tschakert (USA), Detlef van Vuuren (The Netherlands), Jean-Pascal van Ypersele (Belgium)

\section{Technical Support Unit for the Synthesis Report}

Leo Meyer, Sander Brinkman, Line van Kesteren, Noëmie Leprince-Ringuet, Fijke van Boxmeer

\section{Referencing this report}

IPCC, 2014: Climate Change 2014: Synthesis Report. Contribution of Working Groups I, II and III to the Fifth Assessment Report of the Intergovernmental Panel on Climate Change [Core Writing Team, R.K. Pachauri and L.A. Meyer (eds.)]. IPCC, Geneva, Switzerland, 151 pp. 


\title{
THE INTERGOVERNMENTAL PANEL ON CLIMATE CHANGE
}

\author{
(C) Intergovernmental Panel on Climate Change, 2015
}

First published 2015

ISBN 978-92-9169-143-2

This publication is identical to the report that was approved (Summary for Policymakers) and adopted (longer report) at the 40th session of the Intergovernmental Panel on Climate Change (IPCC) on 1 November 2014 in Copenhagen, Denmark, but with the inclusion of copy-edits and errata that have been corrected prior to this publication. These pre-publication errata are available at: http://www.ipcc.ch.

The designations employed and the presentation of material on maps do not imply the expression of any opinion whatsoever on the part of the Intergovernmental Panel on Climate Change concerning the legal status of any country, territory, city or area or of its authorities, or concerning the delimitation of its frontiers or boundaries.

The mention of specific companies or products does not imply that they are endorsed or recommended by IPCC in preference to others of a similar nature, which are not mentioned or advertised.

The right of publication in print, electronic and any other form and in any language is reserved by the IPCC. Short extracts from this publication may be reproduced without authorization provided that complete source is clearly indicated. Editorial correspondence and requests to publish, reproduce or translate articles in part or in whole should be addressed to:

IPCC

c/o World Meteorological Organization (WMO)

7 bis, avenue de la Paix

Tel.: +41227308208

P.O. Box 2300

Fax: +41227308025

CH 1211 Geneva 2, Switzerland

E-mail: IPCC-Sec@wmo.int

www.ipcc.ch

Cover: Design by Laura Biagioni, IPCC Secretariat, WMO

Photos:

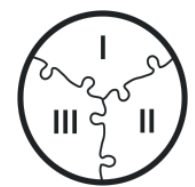

I - Folgefonna glacier on the high plateaus of Sørfjorden, Norway $\left(60^{\circ} 03^{\prime} \mathrm{N}-6^{\circ} 20^{\prime} \mathrm{E}\right)$.

(C) Yann Arthus-Bertrand / Altitude | www.yannarthusbertrand.org | www.goodplanet.org

II - Planting of mangrove seedlings in Funafala, Funafuti Atoll, Tuvalu. @ David J. Wilson

III - China, Shanghai, aerial view. (C) Ocean/Corbis 
Foreword, Preface and Dedication 



\section{Foreword}

The Synthesis Report (SYR) distils and integrates the findings of the three Working Group contributions to the Fifth Assessment Report (AR5) of the Intergovernmental Panel on Climate Change (IPCC), the most comprehensive assessment of climate change undertaken thus far by the IPCC: Climate Change 2013: The Physical Science Basis; Climate Change 2014: Impacts, Adaptation, and Vulnerability; and Climate Change 2014: Mitigation of Climate Change. The SYR also incorporates the findings of two Special Reports on Renewable Energy Sources and Climate Change Mitigation (2011) and on Managing the Risks of Extreme Events and Disasters to Advance Climate Change Adaptation (2011).

The SYR confirms that human influence on the climate system is clear and growing, with impacts observed across all continents and oceans. Many of the observed changes since the 1950s are unprecedented over decades to millennia. The IPCC is now 95 percent certain that humans are the main cause of current global warming. In addition, the SYR finds that the more human activities disrupt the climate, the greater the risks of severe, pervasive and irreversible impacts for people and ecosystems, and long-lasting changes in all components of the climate system. The SYR highlights that we have the means to limit climate change and its risks, with many solutions that allow for continued economic and human development. However, stabilizing temperature increase to below $2^{\circ} \mathrm{C}$ relative to pre-industrial levels will require an urgent and fundamental departure from business as usual. Moreover, the longer we wait to take action, the more it will cost and the greater the technological, economic, social and institutional challenges we will face.

These and the other findings of the SYR have undoubtedly and considerably enhanced our understanding of some of the most critical issues in relation to climate change: the role of greenhouse gas emissions; the severity of potential risks and impacts, especially for the least developed countries and vulnerable communities, given their limited ability to cope; and the options available to us and their underlying requirements to ensure that the effects of climate change remain manageable. As such, the SYR calls for the urgent attention of both policymakers and citizens of the world to tackle this challenge.

The timing of the SYR, which was released on 2nd November 2014 in Copenhagen, was crucial. Policymakers met in December 2014 in Lima at the $20^{\text {th }}$ Conference of Parties under the United Nations Framework Convention on Climate Change (UNFCCC) to prepare the groundwork for the $21^{\text {st }}$ Session in 2015 in Paris, when they have been tasked with concluding a new agreement to deal with climate change. It is our hope that the scientific findings of the SYR will be the basis of their motivation to find the way to a global agreement which can keep climate change to a manageable level, as the SYR gives us the knowledge to make informed choices, and enhances our vital understanding of the rationale for action - and the serious implications of inaction. Ignorance can no longer be an excuse for tergiversation.

As an intergovernmental body jointly established in 1988 by the World Meteorological Organization (WMO) and the United Nations Environment Programme (UNEP), the Intergovernmental Panel on Climate Change (IPCC) has provided policymakers with the most authoritative and objective scientific and technical assessments in this field. Beginning in 1990, this series of IPCC Assessment Reports, Special Reports, Technical Papers, Methodology Reports and other products have become standard works of reference.

The SYR was made possible thanks to the voluntary work, dedication and commitment of thousands of experts and scientists from around the globe, representing a range of views and disciplines. We would like to express our deep gratitude to all the members of the Core Writing Team of the SYR, members of the Extended Writing Team, and the Review Editors, all of whom enthusiastically took on the huge challenge of producing an outstanding SYR on top of the other tasks they had already committed to during the AR5 cycle. We would also like to thank the staff of the Technical Support Unit of the SYR and the IPCC Secretariat for their dedication in organizing the production of this IPCC report.

We also wish to acknowledge and thank the governments of the IPCC member countries for their support of scientists in developing this report, and for their contributions to the IPCC Trust Fund to provide the essentials for participation of experts from developing countries and countries with economies in transition. We would like to express our appreciation to the government of Wallonia (Belgium) for hosting the Scoping Meeting of the SYR, to the governments of Norway, the Netherlands, Germany and Malaysia for hosting drafting sessions of the SYR, and to the government of Denmark for hosting the 40th Session of the IPCC where the SYR was approved. The generous financial support from the governments of Norway and the Netherlands, from the Korea Energy Economics Institute, and the in-kind support by the Netherlands Environmental Assessment Agency and The Energy and Resources Institute, New Delhi (India), enabled the smooth operation of the Technical Support Unit of the SYR. This is gratefully acknowledged.

We would particularly like to express our thanks to Dr Rajendra K. Pachauri, Chairman of the IPCC, for his leadership and constant guidance throughout the production of this report.

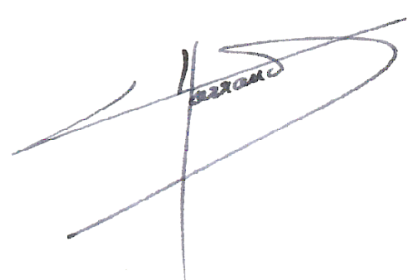

Michel Jarraud

Secretary General

World Meteorological Organization

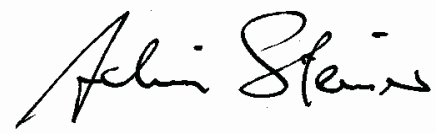

Achim Steiner

Executive Director

United Nations Environmental Programme 



\section{Preface}

The Synthesis Report (SYR), constituting the final product of the Fifth Assessment Report (AR5) of the Intergovernmental Panel on Climate Change (IPCC), is published under the title Climate Change 2014. This report distils, synthesizes and integrates the key findings of the three Working Group contributions - The Physical Science Basis, Impacts, Adaptation, and Vulnerability and Mitigation of Climate Change - to the AR5 in a concise document for the benefit of decision makers in the government, the private sector as well as the public at large. The SYR also draws on the findings of the two Special Reports brought out in 2011 dealing with Renewable Energy Sources and Climate Change Mitigation, and Managing the Risks of Extreme Events and Disasters to Advance Climate Change Adaptation. The SYR, therefore, is a comprehensive up-to-date compilation of assessments dealing with climate change, based on the most recent scientific, technical and socio-economic literature in the field.

\section{Scope of the Report}

This document is the result of coordinated and carefully connected cross Working Group efforts to ensure coherent and comprehensive information on various aspects related to climate change. This SYR includes a consistent evaluation and assessment of uncertainties and risks; integrated costing and economic analysis; regional aspects; changes, impacts and responses related to water and earth systems, the carbon cycle including ocean acidification, cryosphere and sea level rise; as well as treatment of mitigation and adaptation options within the framework of sustainable development. Through the entire length of the SYR, information is also provided relevant to Article 2, the ultimate objective of the United Nations Framework Convention on Climate Change (UNFCCC).

Other aspects of climate change covered in this report include direct impacts of climate change on natural systems as well as both direct and indirect impacts on human systems, such as human health, food security and security of societal conditions. By embedding climate change risk and issues of adaptation and mitigation within the framework of sustainable development, the SYR also highlights the fact that nearly all systems on this planet would be affected by the impacts of a changing climate, and that it is not possible to draw boundaries around climate change, its associated risks and impacts on the one hand and on the other, development which meets the needs of the present generation without compromising the ability of future generations to meet their own needs. The Report, therefore, also focuses on connections between these aspects and provides information on how climate change overlaps with and mainstreams into other developmental issues.

\section{Structure}

The Report comprises a Summary for Policymakers (SPM) and a longer report from which the SPM is derived, as well as annexes. Even though the SPM follows a structure and sequence similar to that in the longer report, some specific issues covered under more than one topic of the longer report are summarized in one particular section of the SPM. Each paragraph of the SPM contains references to the respective text in the longer report. In turn, the latter contains extensive references to relevant chapters of the underlying Working Group Reports or the two Special Reports mentioned above. The SYR is essentially self-contained, and its SPM includes the most policy relevant material drawn from the longer report and the entire AR5.

All the three contributions to the AR5 including each Summary for Policymakers, each Technical Summary, frequently asked questions as well as the Synthesis Report in all official UN languages are available online on the IPCC website and in electronic offline versions. In these electronic versions, references in the SYR to relevant parts of the underlying material are provided as hyperlinks, thereby enabling the reader to easily find further scientific, technical and socio-economic information. A user guide, glossary of terms used and listing of acronyms, authors, Review Editors and Expert Reviewers are provided in the annexes to this report.

To facilitate access to the findings of the SYR for a wide readership and to enhance their usability for stakeholders, each section of the SPM carries highlighted headline statements. Taken together, these 21 headline statements provide an overarching summary in simple and completely non-technical language for easy assimilation by readers from different walks of life. These headline statements have been crafted by the authors of the Report, and approved by the member governments of the IPCC.

The longer report is structured around four topic headings as mandated by the Panel:

Observed changes and their causes (Topic 1) integrates new information from the three Working Groups on observed changes in the climate system, including changes in the atmosphere, oceans, cryosphere and sea level; recent and past drivers and human influences affecting emission drivers; observed impacts, including changes in extreme weather and climate events; and attribution of climate changes and impacts.

Future climate changes, risks and impacts (Topic 2) presents information about future climate change, risks and impacts. It integrates information about key drivers of future climate, the relationship between cumulative emissions and temperature change, and projected changes in the climate system in the $21^{\text {st }}$ century and beyond. It assesses future risks and impacts caused by a changing climate and the interaction of climate-related and other hazards. It provides information about longterm changes including sea-level rise and ocean acidification, and the risk of irreversible and abrupt changes.

Future Pathways for Adaptation, Mitigation and Sustainable Development (Topic 3) addresses future pathways for adaptation and mitigation as complementary strategies for reducing and managing the risks of climate change and assesses their interaction with sustainable development. It describes analytical approaches for effective 
decision-making and differences in risks of climate change, adaptation and mitigation in terms of timescale, magnitude and persistence. It analyses the characteristics of adaptation and mitigation pathways, and associated challenges, limits and benefits, including for different levels of future warming.

Adaptation and Mitigation (Topic 4) brings together information from Working Groups II and III on specific adaptation and mitigation options, including environmentally sound technologies and infrastructure, sustainable livelihoods, behaviour and lifestyle choices. It describes common enabling factors and constraints, and policy approaches, finance and technology on which effective response measures depend. It shows opportunities for integrated responses and links adaptation and mitigation with other societal objectives.

\section{Process}

The SYR of the AR5 of the IPCC has been prepared in accordance with the procedures of the IPCC to ensure adequate effort and rigor being achieved in the process. For the AR5 the preparation of the SYR was taken in hand a year earlier than was the case with the Fourth Assessment Report (AR4) - while the Working Group Reports were still being completed - with a view to enhancing integration and ensuring adequate synthesis. A scoping meeting specifically for proposing the detailed outline of the AR5 Synthesis Report was held in Liège, Belgium in August, 2010, and the outline produced in that meeting was approved by the Panel in October, 2010 in Busan, Republic of Korea. In accordance with IPCC procedures, the IPCC Chair in consultation with the Co-Chairs of the Working Groups nominated authors for the Core Writing Team (CWT) of the SYR and a total of 45 CWT members and 9 Review Editors were selected and accepted by the IPCC Bureau in March, 2012. In addition, 14 Extended Writing Team (EWT) authors were selected by the CWT with the approval of the Chair of the IPCC, and this latter group contributed substantially to the material and the text provided in this report. During evolution of the contents of the SYR the IPCC Bureau was approached and it approved the inclusion of 6 additional CWT members and an additional Review Editor. This further enhanced and deepened the expertise required for the preparation of the Report. The final draft report which has undergone a combined review by experts and governments was submitted to the 40th Session of the IPCC, held from 27 October to 1 November 2014 in Copenhagen, Denmark, where governments approved the SPM line by line and adopted the longer report section by section.

\section{Acknowledgements}

Our profound gratitude and deep indebtedness goes to the members of the Core Writing Team and the substantial help from the Extended Writing Team members, for their tireless efforts, expertise, and amazing level of dedication throughout the production of the SYR. The SYR could not have been completed successfully without their inspirational commitment to excellence and integrity, and their meticulous attention to detail. We also wish to thank the Review Editors for their invaluable help ensuring that the SYR provides a balanced and complete assessment of current information relevant to climate change. Their role was crucial to ensure transparency of the process which the IPCC can pride itself on. Our thanks go also to all authors of the AR5 and the two Special Reports because without their careful assessment of the huge body of literature on various aspects of climate change and their comments on the draft report, the preparation of the SYR would not have been possible.

Throughout the AR5, we benefitted greatly from the wisdom and insight of our colleagues in the IPCC leadership, especially Dr Thomas Stocker and Dr Qin Dahe, Working Group I Co-Chairs; Dr Chris Field and Dr Vicente Barros, Working Group II Co-Chairs; and Dr Ottmar Edenhofer, Dr Ramón Pichs-Madruga and Dr Youba Sokona, Working Group III Co-Chairs. Their cooperation on issues related to knowledge from the reports of all three Working Groups was a definite asset for the production of a high-quality final document.

We also wish to thank Fredolin Tangang, David Wratt, Eduardo Calvo, Jose Moreno, Jim Skea and Suzana Kahn Ribeiro, who acted as Review Editors during the Approval Session of the SYR, ensuring that the edits made to the SPM during the Session were correctly reflected in the longer report. Their important work guaranteed the high level of trust between the scientists and the governments, enabling them to work smoothly in symbiosis, which is a unique feature of the IPCC and its credibility.

We extend our deep appreciation of the enthusiasm, dedication and professional contributions of Gian-Kasper Plattner, Melinda Tignor and Judith Boschung from the Technical Support Unit of Working Group I, Katie Mach and Eren Bilir from the Technical Support Unit of Working Group II, Ellie Farahani, Jussi Savolainen and Steffen Schlömer from the Technical Support Unit of Working Group III, and Gerrit Hansen from the Potsdam Institute for Climate Impact Research during the Approval Session of the SYR, working as a team with the Technical Support Unit of the SYR, which was indispensable in the successful outcomes of the Session. A special thanks goes to Adrien Michel from the Technical Support Unit of Working Group I for his work on the SYR figures.

Our thanks go to Leo Meyer, Head of the Technical Support Unit of the Synthesis Report, and the members of the Technical Support Unit Sander Brinkman, Line van Kesteren, Noemie Leprince-Ringuet and Fijke van Boxmeer for their capacity to expand their strengths and carry out the mammoth task of coordinating the development and production of the SYR. Each one of them put in tireless efforts, displaying deep commitment and dedication to ensure the production of an outstanding SYR.

We would like to acknowledge the work and innumerable tasks performed in support of the preparation, release and publication of the Report by the staff of the IPCC Secretariat: Gaetano Leone, Carlos Martin-Novella, Jonathan Lynn, Brenda Abrar-Milani, Jesbin Baidya, Laura Biagioni, Mary Jean Burer, Annie Courtin, Judith Ewa, Joelle Fernandez, Nina Peeva, Sophie Schlingemann, Amy Smith and Werani Zabula. Thanks are also due to Francis Hayes and Elhousseine Gouaini for acting as conference officers at the approval Session.

We are appreciative of the member governments of the IPCC who graciously hosted the SYR scoping meeting, four of our Core Writing Meetings and the 40th Session of the IPCC: Belgium, Norway, The Netherlands, Germany, Malaysia and Denmark. We express our thanks 
to the governments, WMO, UNEP and the UNFCCC for their contributions to the Trust Fund which supported various elements of expenditure. We wish to particularly thank the Governments of Norway and The Netherlands, and the Korea Energy Economics Institute for their generous financial support of the SYR Technical Support Unit, and The Netherlands Environmental Assessment Agency PBL and The Energy and Resources Institute, New Delhi, for their in-kind support of the SYR Technical Support Unit. We also acknowledge the support of IPCC's parent organizations, UNEP and WMO, and particularly WMO for hosting the IPCC Secretariat and our first Core Writing Team meeting. May we convey our deep gratitude to the UNFCCC for their cooperation at various stages of this enterprise and for the prominence they give to our work in several appropriate fora.

\section{Dahann}

R.K. Pachauri

Chairman of the IPCC

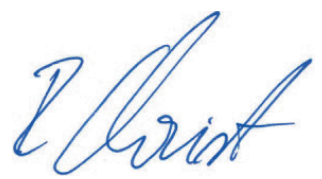

Renate Christ

Secretary of the IPCC 



\section{Dedication}

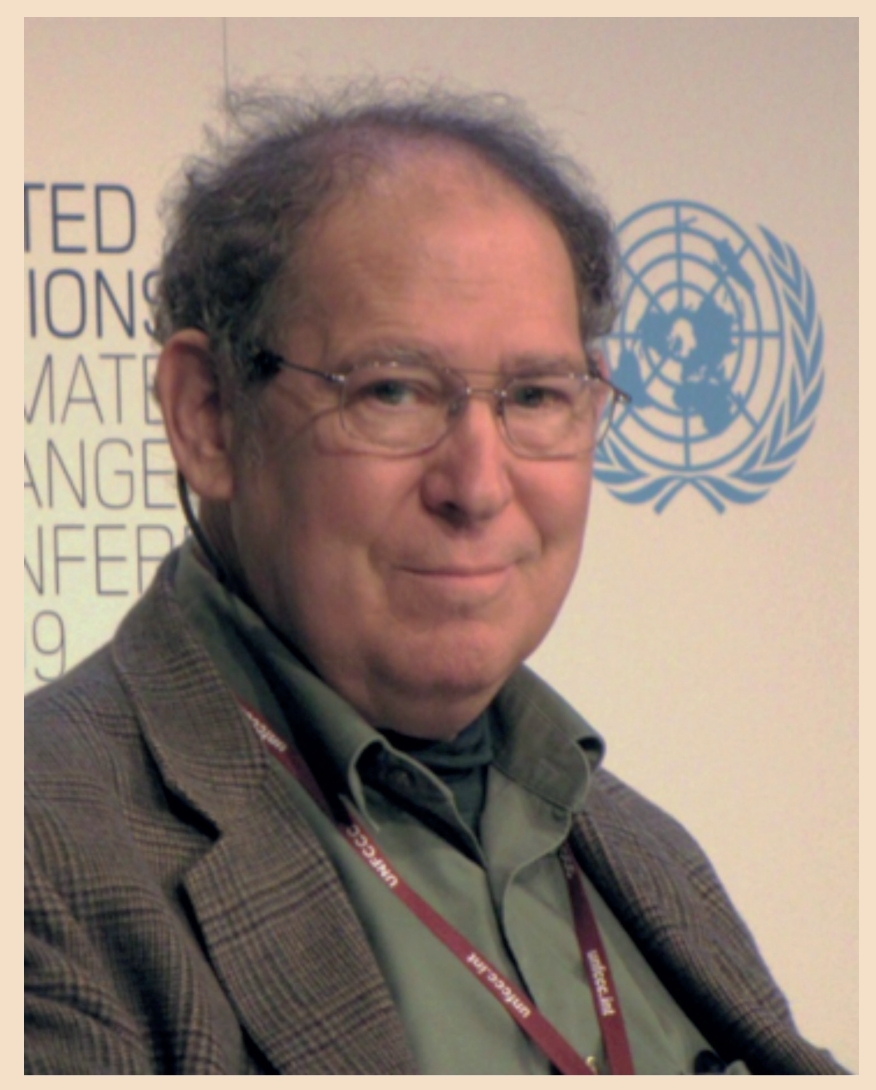

\section{Stephen H. Schneider \\ (11 February 1945 - 19 July 2010)}

The Synthesis Report of the Fifth Assessment Report of the Intergovernmental Panel on Climate Change (IPCC) is dedicated to the memory of Stephen H. Schneider, one of the foremost climate scientists of our time.

Steve Schneider, born in New York, trained as a plasma physicist, embraced scholarship in the field of climate science almost 40 years ago and continued his relentless efforts creating new knowledge in the field and informing policymakers and the public at large on the growing problem of climate change and solutions for dealing with it. At all times Steve Schneider remained intrepid and forthright in expressing his views. His convictions were driven by the strength of his outstanding scientific expertise. He was highly respected as Founding Editor of the interdisciplinary journal Climatic Change and authored hundreds of books and papers, many of which were co-authored with scientists from diverse disciplines. His association with the IPCC began with the First Assessment Report which was published in 1990, and which played a major role in the scientific foundation of the UN Framework Convention on Climate Change. Subsequently, he was Lead Author, Coordinating Lead Author and Expert Reviewer for various Assessment Reports and a member of the Core Writing Team for the Synthesis Report of the Fourth Assessment Report. His life and accomplishments have inspired and motivated members of the Core Writing Team of this Report. Steve Schneider's knowledge was a rare synthesis of several disciplines which are an essential part of the diversity inherent in climate science. 



\section{Contents}

Preface

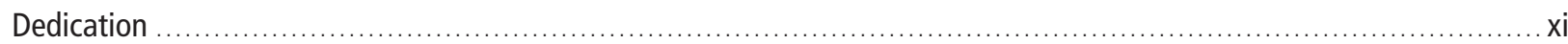

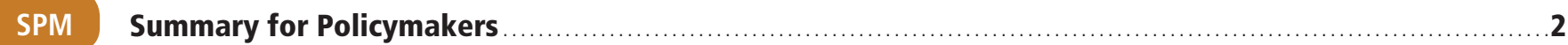

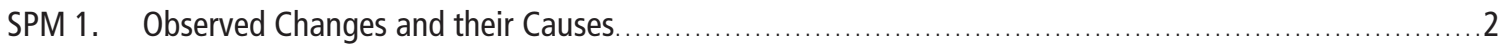

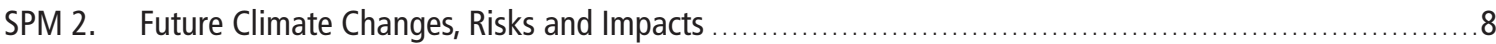

SPM 3. Future Pathways for Adaptation, Mitigation and Sustainable Development ............................ 17

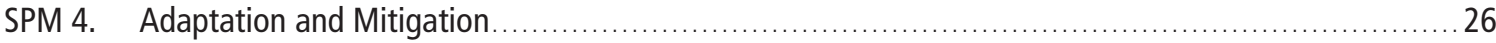

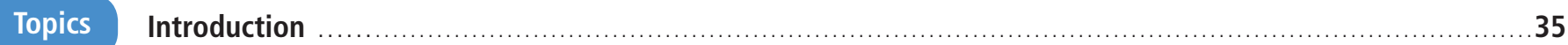

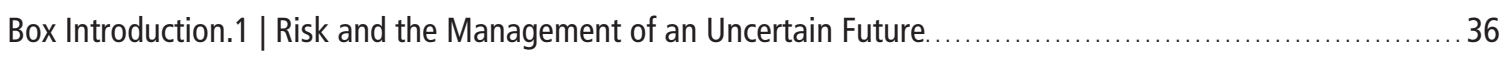

Box Introduction.2 | Communicating the Degree of Certainty in Assessment Findings .............................. 37

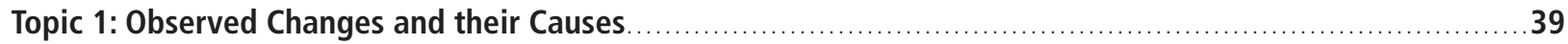

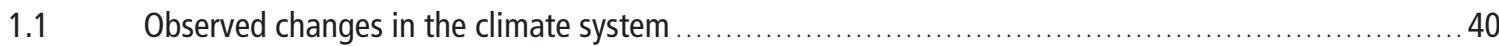

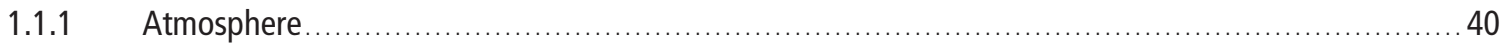

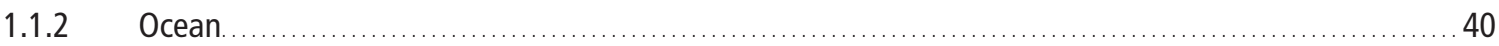

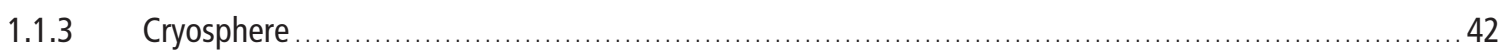

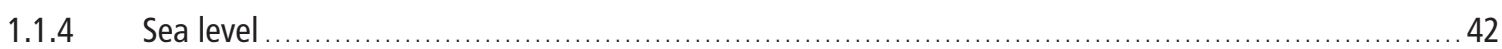

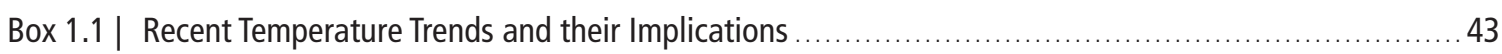

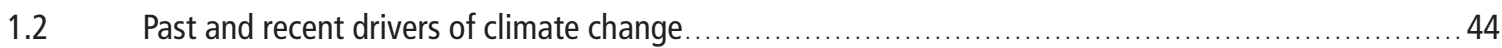

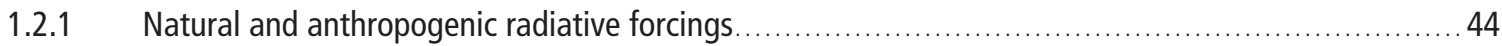

1.2.2 Human activities affecting emission drivers............................................................... 45

1.3 Attribution of climate changes and impacts ........................................................... 47

1.3.1 Attribution of climate changes to human and natural influences on the climate system ................ 48

1.3.2 Observed impacts attributed to climate change ..................................................... 49

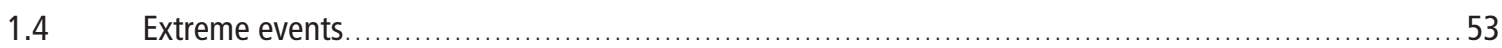

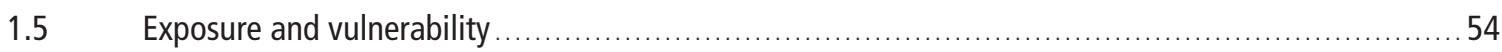

1.6 Human responses to climate change: adaptation and mitigation .......................................5 54

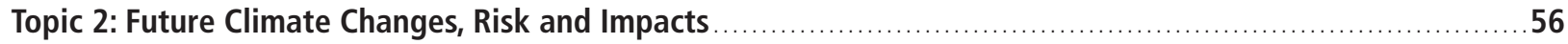

2.1 Key drivers of future climate and the basis on which projections are made ............................ 56

Box 2.1 | Advances, Confidence and Uncertainty in Modelling the Earth's Climate System ........................ 56

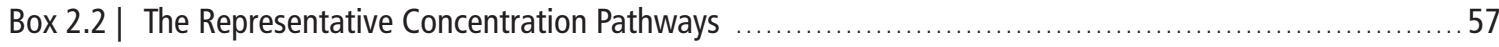




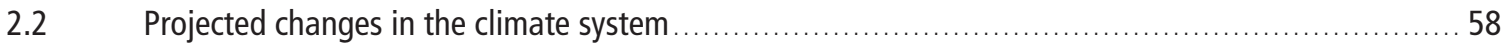

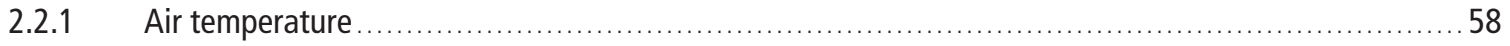

Box 2.3 | Models and Methods for Estimating Climate Change Risks, Vulnerability and Impacts...................58

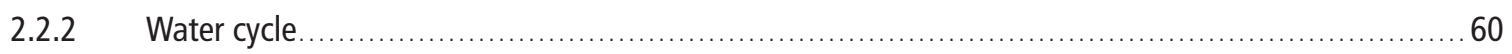

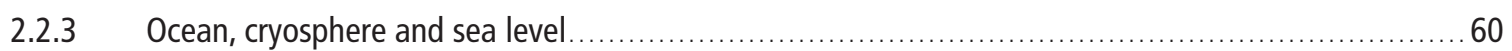

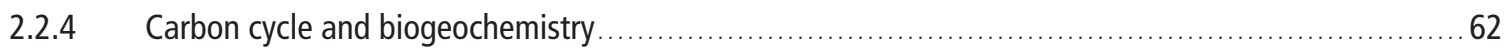

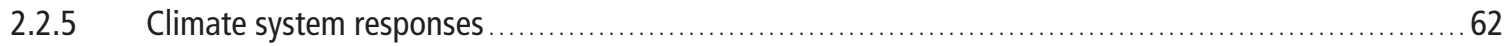

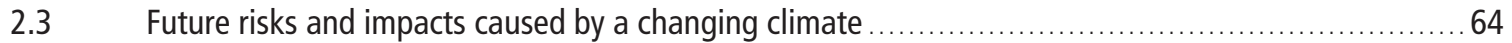

2.3.1 Ecosystems and their services in the oceans, along coasts, on land and in freshwater...................67

2.3.2 Water, food and urban systems, human health, security and livelihoods ..............................67

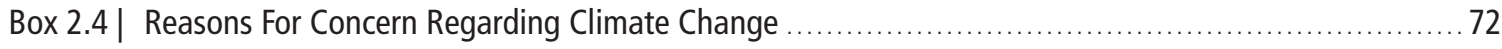

2.4 Climate change beyond 2100 , irreversibility and abrupt changes ..................................... 73

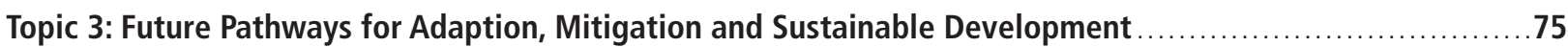

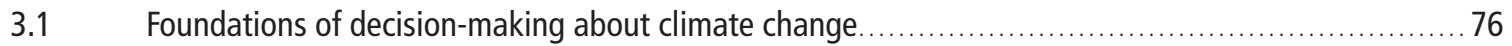

3.2 Climate change risks reduced by adaptation and mitigation ............................................ 77

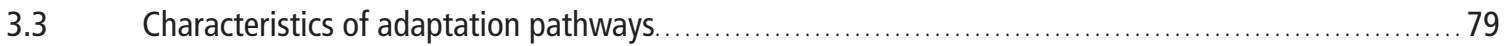

Box 3.1 | The Limits of the Economic Assessment of Climate Change Risks ...................................... 79

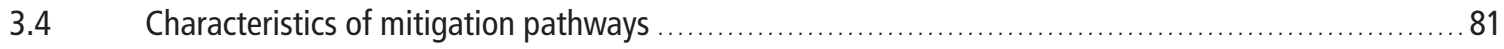

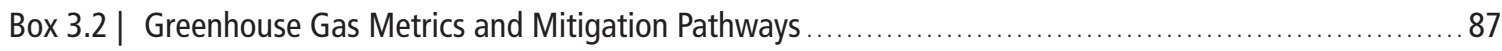

Box 3.3 | Carbon Dioxide Removal and Solar Radiation Management Geoengineering Technologies-

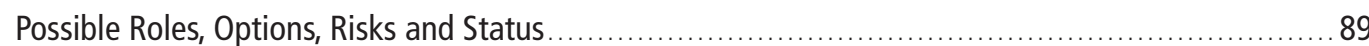

3.5 Interaction among mitigation, adaptation and sustainable development ............................90

Box 3.4 | Co-benefits and Adverse Side effects .................................................................. 91

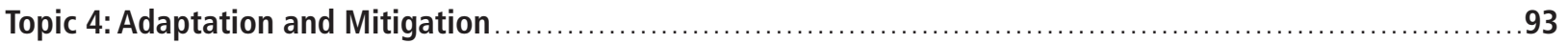

4.1 Common enabling factors and constraints for adaptation and mitigation responses .................... 94

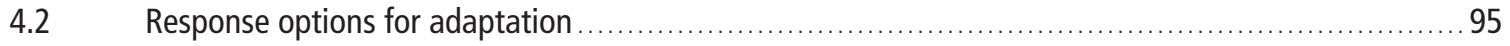

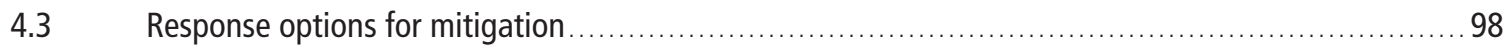

4.4 Policy approaches for adaptation and mitigation, technology and finance ........................... 102

4.4.1 International and regional cooperation on adaptation and mitigation ................................. 102

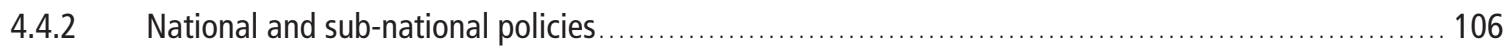

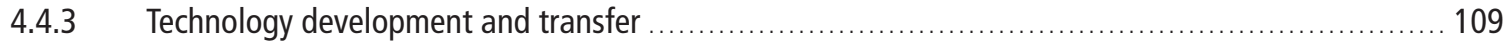

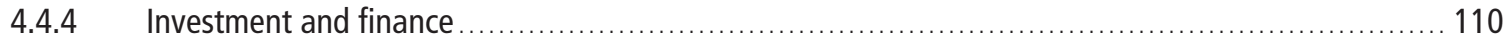

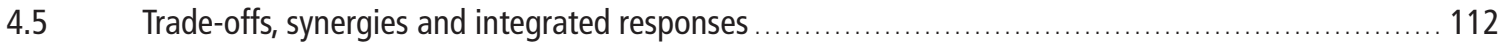




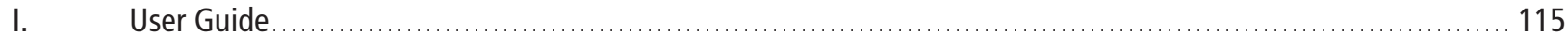

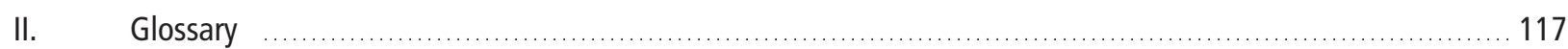

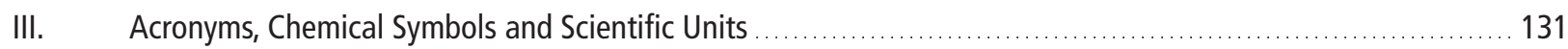

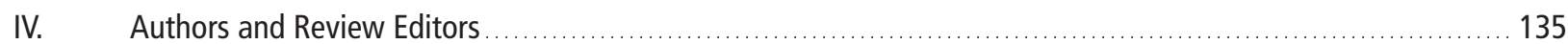

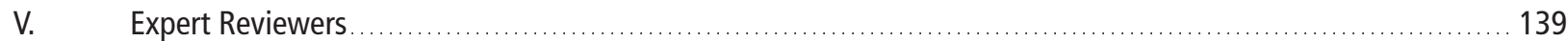

VI. Publications by the Intergovernmental Panel on Climate Change ........................................... 143

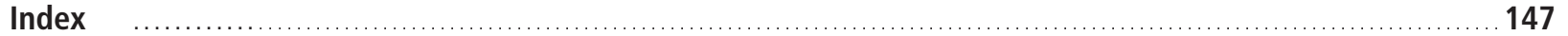




\section{Sources cited in this Synthesis Report}

References for material contained in this report are given in italicized curly brackets \{\} at the end of each paragraph.

In the Summary for Policymakers, the references refer to the numbers of the sections, figures, tables and boxes in the underlying Introduction and Topics of this Synthesis Report.

In the Introduction and Topics of the longer report, the references refer to the contributions of the Working Groups I, II and III (WGI, WGII, WGIII) to the Fifth Assessment Report and other IPCC Reports (in italicized curly brackets), or to other sections of the Synthesis Report itself (in round brackets).

The following abbreviations have been used:

SPM: Summary for Policymakers

TS: $\quad$ Technical Summary

ES: $\quad$ Executive Summary of a chapter

Numbers denote specific chapters and sections of a report.

Other IPCC reports cited in this Synthesis Report:

SREX: Special Report on Managing the Risks of Extreme Events and Disasters to Advance Climate Change Adaptation

SRREN: Special Report on Renewable Energy Sources and Climate Change Mitigation

AR4: Fourth Assessment Report 


\section{Introduction}

This Synthesis Report is based on the reports of the three Working Groups of the Intergovernmental Panel on Climate Change (IPCC), including relevant Special Reports. It provides an integrated view of climate change as the final part of the IPCC's Fifth Assessment Report (AR5).

This summary follows the structure of the longer report which addresses the following topics: Observed changes and their causes; Future climate change, risks and impacts; Future pathways for adaptation, mitigation and sustainable development; Adaptation and mitigation.

In the Synthesis Report, the certainty in key assessment findings is communicated as in the Working Group Reports and Special Reports. It is based on the author teams' evaluations of underlying scientific understanding and is expressed as a qualitative level of confidence (from very low to very high) and, when possible, probabilistically with a quantified likelihood (from exceptionally unlikely to virtually certain) ${ }^{1}$. Where appropriate, findings are also formulated as statements of fact without using uncertainty qualifiers.

This report includes information relevant to Article 2 of the United Nations Framework Convention on Climate Change (UNFCCC).

\section{SPM 1. Observed Changes and their Causes}

Human influence on the climate system is clear, and recent anthropogenic emissions of greenhouse gases are the highest in history. Recent climate changes have had widespread impacts on human and natural systems. \{1\}

\section{SPM 1.1 Observed changes in the climate system}

Warming of the climate system is unequivocal, and since the 1950s, many of the observed changes are unprecedented over decades to millennia. The atmosphere and ocean have warmed, the amounts of snow and ice have diminished, and sea level has risen. $\{1.1\}$

Each of the last three decades has been successively warmer at the Earth's surface than any preceding decade since 1850 . The period from 1983 to 2012 was likely the warmest 30-year period of the last 1400 years in the Northern Hemisphere, where such assessment is possible (medium confidence). The globally averaged combined land and ocean surface temperature data as calculated by a linear trend show a warming of 0.85 [0.65 to 1.06$]^{\circ} \mathrm{C}^{2}$ over the period 1880 to 2012 , when multiple independently produced datasets exist (Figure SPM.1a). \{1.1.1, Figure 1.1\}

In addition to robust multi-decadal warming, the globally averaged surface temperature exhibits substantial decadal and interannual variability (Figure SPM.1a). Due to this natural variability, trends based on short records are very sensitive to the beginning and end dates and do not in general reflect long-term climate trends. As one example, the rate of warming over

Each finding is grounded in an evaluation of underlying evidence and agreement. In many cases, a synthesis of evidence and agreement supports an assignment of confidence. The summary terms for evidence are: limited, medium or robust. For agreement, they are low, medium or high. A level of confidence is expressed using five qualifiers: very low, low, medium, high and very high, and typeset in italics, e.g., medium confidence. The following terms have been used to indicate the assessed likelihood of an outcome or a result: virtually certain 99-100\% probability, very likely $90-100 \%$, likely $66-100 \%$, about as likely as not 33-66\%, unlikely $0-33 \%$, very unlikely $0-10 \%$, exceptionally unlikely $0-1 \%$. Additional terms (extremely likely $95-100 \%$, more likely than not $>50-100 \%$, more unlikely than likely $0-<50 \%$, extremely unlikely $0-5 \%$ ) may also be used when appropriate. Assessed likelihood is typeset in italics, e.g., very likely. See for more details: Mastrandrea, M.D., C.B. Field, T.F. Stocker, O. Edenhofer, K.L. Ebi, D.J. Frame, H. Held, E. Kriegler, K.J. Mach, P.R. Matschoss, G.-K. Plattner, G.W. Yohe and F.W. Zwiers, 2010: Guidance Note for Lead Authors of the IPCC Fifth Assessment Report on Consistent Treatment of Uncertainties, Intergovernmental Panel on Climate Change (IPCC), Geneva, Switzerland, 4 pp.

2 Ranges in square brackets or following ' \pm ' are expected to have a $90 \%$ likelihood of including the value that is being estimated, unless otherwise stated. 
(a) Globally averaged combined land and ocean surface temperature anomaly

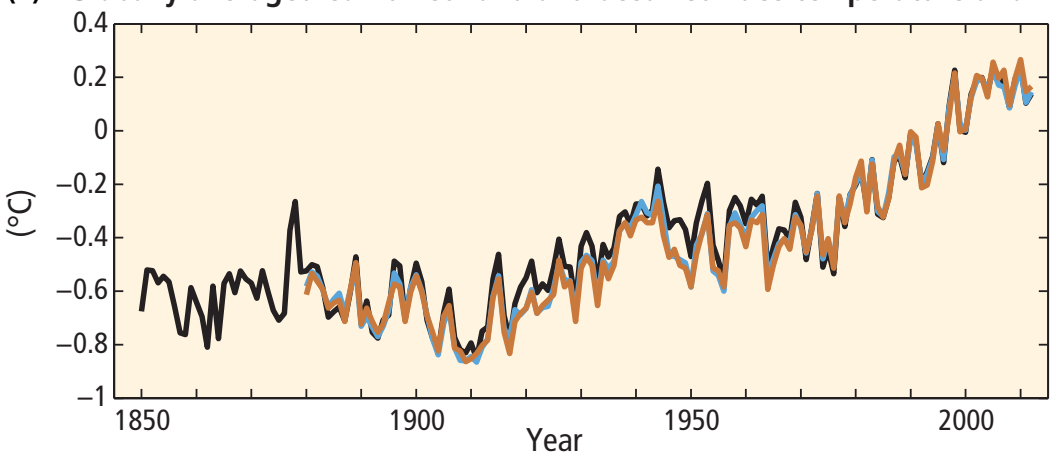

(b)

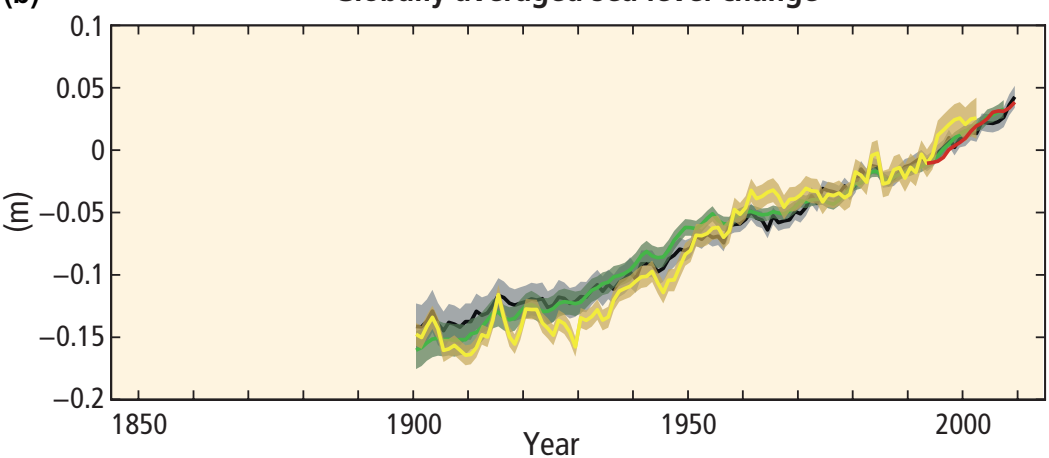

(c)

Globally averaged greenhouse gas concentrations

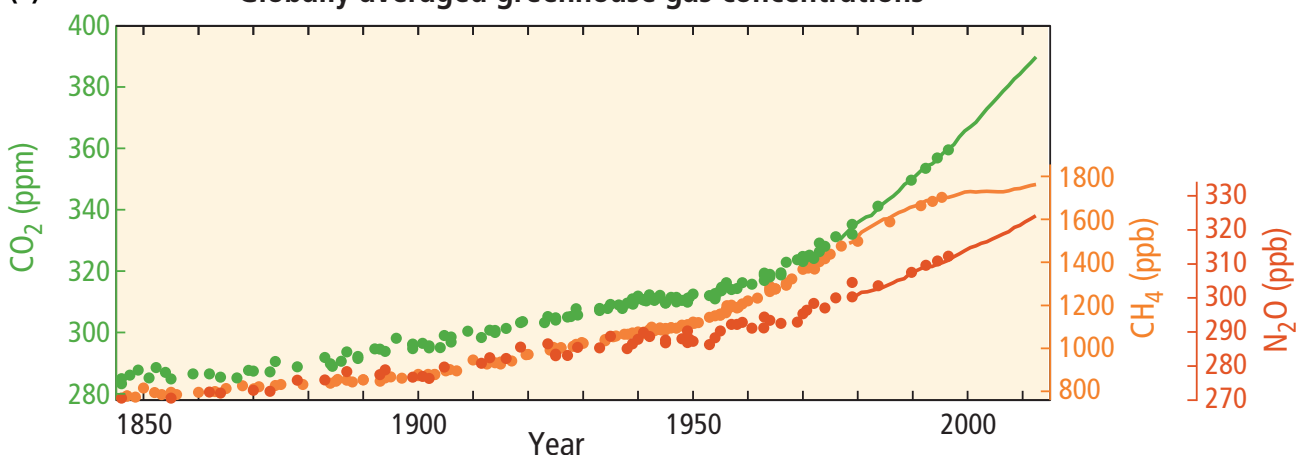

(d)

Global anthropogenic $\mathrm{CO}_{2}$ emissions

(d) Quantitative information of $\mathrm{CH}_{4}$ and $\mathrm{N}_{2} \mathrm{O}$ emission time series from 1850 to 1970 is limited

\section{Cumulative $\mathrm{CO}_{2}$} emissions
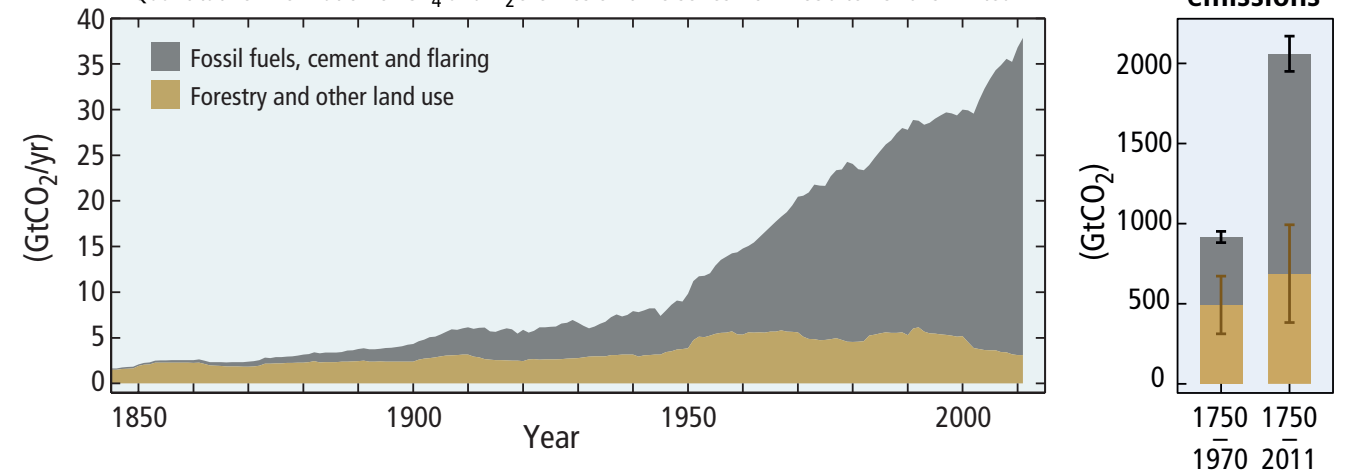

Figure SPM.1 | The complex relationship between the observations (panels a, b, c, yellow background) and the emissions (panel d, light blue background) is addressed in Section 1.2 and Topic 1. Observations and other indicators of a changing global climate system. Observations: (a) Annually and globally averaged combined land and ocean surface temperature anomalies relative to the average over the period 1986 to 2005. Colours indicate different data sets. (b) Annually and globally averaged sea level change relative to the average over the period 1986 to 2005 in the longest-running dataset. Colours indicate different data sets. All datasets are aligned to have the same value in 1993, the first year of satellite altimetry data (red). Where assessed, uncertainties are indicated by coloured shading. (c) Atmospheric concentrations of the greenhouse gases carbon dioxide $\left(\mathrm{CO}_{2}\right.$, green), methane $\left(\mathrm{CH}_{4}\right.$, orange) and nitrous oxide $\left(\mathrm{N}_{2} \mathrm{O}\right.$, red) determined from ice core data (dots) and from direct atmospheric measurements (lines). Indicators: (d) Global anthropogenic $\mathrm{CO}_{2}$ emissions from forestry and other land use as well as from burning of fossil fuel, cement production and flaring. Cumulative emissions of $\mathrm{CO}_{2}$ from these sources and their uncertainties are shown as bars and whiskers, respectively, on the right hand side. The global effects of the accumulation of $\mathrm{CH}_{4}$ and $\mathrm{N}_{2} \mathrm{O}$ emissions are shown in panel c. Greenhouse gas emission data from 1970 to 2010 are shown in Figure SPM.2. \{Figures 1.1, 1.3, 1.5\} 
the past 15 years (1998-2012; $0.05[-0.05 \text { to } 0.15]^{\circ} \mathrm{C}$ per decade), which begins with a strong El Niño, is smaller than the rate calculated since 1951 (1951-2012; 0.12 [0.08 to 0.14$]^{\circ} \mathrm{C}$ per decade). \{1.1.1, Box 1.1\}

Ocean warming dominates the increase in energy stored in the climate system, accounting for more than $90 \%$ of the energy accumulated between 1971 and 2010 (high confidence), with only about 1\% stored in the atmosphere. On a global scale, the ocean warming is largest near the surface, and the upper $75 \mathrm{~m}$ warmed by 0.11 [0.09 to $0.13{ }^{\circ} \mathrm{C}$ per decade over the period 1971 to 2010. It is virtually certain that the upper ocean $(0-700 \mathrm{~m})$ warmed from 1971 to 2010, and it likely warmed between the 1870 s and 1971. \{1.1.2, Figure 1.2\}

Averaged over the mid-latitude land areas of the Northern Hemisphere, precipitation has increased since 1901 (medium confidence before and high confidence after 1951). For other latitudes, area-averaged long-term positive or negative trends have low confidence. Observations of changes in ocean surface salinity also provide indirect evidence for changes in the global water cycle over the ocean (medium confidence). It is very likely that regions of high salinity, where evaporation dominates, have become more saline, while regions of low salinity, where precipitation dominates, have become fresher since the 1950s. $\{1.1 .1,1.1 .2\}$

Since the beginning of the industrial era, oceanic uptake of $\mathrm{CO}_{2}$ has resulted in acidification of the ocean; the $\mathrm{pH}$ of ocean surface water has decreased by 0.1 (high confidence), corresponding to a $26 \%$ increase in acidity, measured as hydrogen ion concentration. $\{1.1 .2\}$

Over the period 1992 to 2011, the Greenland and Antarctic ice sheets have been losing mass (high confidence), likely at a larger rate over 2002 to 2011. Glaciers have continued to shrink almost worldwide (high confidence). Northern Hemisphere spring snow cover has continued to decrease in extent (high confidence). There is high confidence that permafrost temperatures have increased in most regions since the early 1980s in response to increased surface temperature and changing snow cover. $\{1.1 .3\}$

The annual mean Arctic sea-ice extent decreased over the period 1979 to 2012, with a rate that was very likely in the range 3.5 to $4.1 \%$ per decade. Arctic sea-ice extent has decreased in every season and in every successive decade since 1979, with the most rapid decrease in decadal mean extent in summer (high confidence). It is very likely that the annual mean Antarctic sea-ice extent increased in the range of 1.2 to $1.8 \%$ per decade between 1979 and 2012. However, there is high confidence that there are strong regional differences in Antarctica, with extent increasing in some regions and decreasing in others. $\{1.1 .3$, Figure 1.1\}

Over the period 1901 to 2010, global mean sea level rose by 0.19 [0.17 to 0.21 m (Figure SPM.1b). The rate of sea level rise since the mid-19th century has been larger than the mean rate during the previous two millennia (high confidence). \{1.1.4, Figure 1.1\}

\section{SPM 1.2 Causes of climate change}

Anthropogenic greenhouse gas emissions have increased since the pre-industrial era, driven largely by economic and population growth, and are now higher than ever. This has led to atmospheric concentrations of carbon dioxide, methane and nitrous oxide that are unprecedented in at least the last 800,000 years. Their effects, together with those of other anthropogenic drivers, have been detected throughout the climate system and are extremely likely to have been the dominant cause of the observed warming since the mid-20th century. $\{1.2,1.3 .1\}$

Anthropogenic greenhouse gas (GHG) emissions since the pre-industrial era have driven large increases in the atmospheric concentrations of carbon dioxide $\left(\mathrm{CO}_{2}\right)$, methane $\left(\mathrm{CH}_{4}\right)$ and nitrous oxide $\left(\mathrm{N}_{2} \mathrm{O}\right)$ (Figure SPM.1c). Between 1750 and 2011, cumulative anthropogenic $\mathrm{CO}_{2}$ emissions to the atmosphere were $2040 \pm 310 \mathrm{GtCO}_{2}$. About $40 \%$ of these emissions have remained in the atmosphere $\left(880 \pm 35 \mathrm{GtCO}_{2}\right)$; the rest was removed from the atmosphere and stored on land (in plants and soils) and in the ocean. The ocean has absorbed about $30 \%$ of the emitted anthropogenic $\mathrm{CO}_{2}$, causing ocean acidification. About half of the anthropogenic $\mathrm{CO}_{2}$ emissions between 1750 and 2011 have occurred in the last 40 years (high confidence) (Figure SPM.1d). \{1.2.1, 1.2.2\} 
Total annual anthropogenic GHG emissions by gases 1970-2010

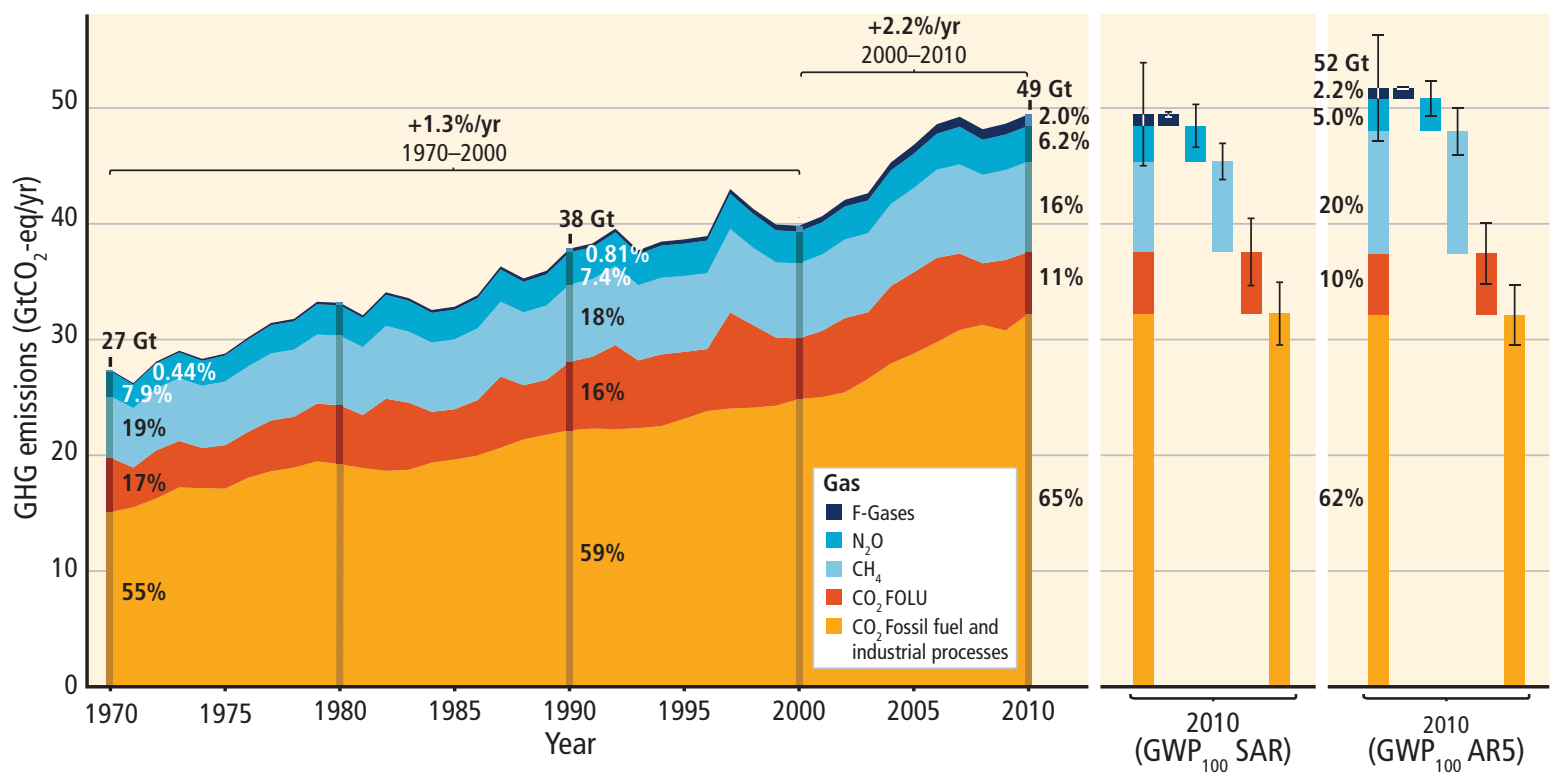

Figure SPM.2 | Total annual anthropogenic greenhouse gas (GHG) emissions (gigatonne of $\mathrm{CO}_{2}$-equivalent per year, $\mathrm{GtCO}_{2}$-eq/yr) for the period 1970 to 2010 by gases: $\mathrm{CO}_{2}$ from fossil fuel combustion and industrial processes; $\mathrm{CO}_{2}$ from Forestry and Other Land Use (FOLU); methane $\left(\mathrm{CH}_{4}\right)$; nitrous oxide $\left(\mathrm{N}_{2} \mathrm{O}\right)$; fluorinated gases covered under the Kyoto Protocol (F-gases). Right hand side shows 2010 emissions, using alternatively $\mathrm{CO}_{2}$-equivalent emission weightings based on IPCC Second Assessment Report (SAR) and AR5 values. Unless otherwise stated, $\mathrm{CO}_{2}$-equivalent emissions in this report include the basket of Kyoto gases $\left(\mathrm{CO}_{2}, \mathrm{CH}_{4}, \mathrm{~N}_{2} \mathrm{O}\right.$ as well as F-gases) calculated based on 100-year Global Warming Potential (GWP $\left.{ }_{100}\right)$ values from the SAR (see Glossary). Using the most recent GWP 100 values from the AR5 (right-hand bars) would result in higher total annual GHG emissions (52 $\mathrm{GtCO}_{2}$-eq/yr) from an increased contribution of methane, but does not change the long-term trend significantly. \{Figure 1.6, Box 3.2\}

Total anthropogenic GHG emissions have continued to increase over 1970 to 2010 with larger absolute increases between 2000 and 2010, despite a growing number of climate change mitigation policies. Anthropogenic GHG emissions in 2010 have reached $49 \pm 4.5 \mathrm{GtCO}_{2}$-eq/yr ${ }^{3}$. Emissions of $\mathrm{CO}_{2}$ from fossil fuel combustion and industrial processes contributed about $78 \%$ of the total GHG emissions increase from 1970 to 2010, with a similar percentage contribution for the increase during the period 2000 to 2010 (high confidence) (Figure SPM.2). Globally, economic and population growth continued to be the most important drivers of increases in $\mathrm{CO}_{2}$ emissions from fossil fuel combustion. The contribution of population growth between 2000 and 2010 remained roughly identical to the previous three decades, while the contribution of economic growth has risen sharply. Increased use of coal has reversed the long-standing trend of gradual decarbonization (i.e., reducing the carbon intensity of energy) of the world's energy supply (high confidence). $\{1.2 .2\}$

The evidence for human influence on the climate system has grown since the IPCC Fourth Assessment Report (AR4). It is extremely likely that more than half of the observed increase in global average surface temperature from 1951 to 2010 was caused by the anthropogenic increase in GHG concentrations and other anthropogenic forcings together. The best estimate of the human-induced contribution to warming is similar to the observed warming over this period (Figure SPM.3). Anthropogenic forcings have likely made a substantial contribution to surface temperature increases since the mid-20th century over every continental region except Antarctica ${ }^{4}$. Anthropogenic influences have likely affected the global water cycle since 1960 and contributed to the retreat of glaciers since the 1960s and to the increased surface melting of the Greenland ice sheet since 1993. Anthropogenic influences have very likely contributed to Arctic sea-ice loss since 1979 and have very likely made a substantial contribution to increases in global upper ocean heat content $(0-700 \mathrm{~m})$ and to global mean sea level rise observed since the 1970s. $\{1.3$, Figure 1.10\}

Greenhouse gas emissions are quantified as $\mathrm{CO}_{2}$-equivalent $\left(\mathrm{GtCO}_{2}\right.$-eq) emissions using weightings based on the 100-year Global Warming Potentials, using IPCC Second Assessment Report values unless otherwise stated. \{Box 3.2\}

4 For Antarctica, large observational uncertainties result in low confidence that anthropogenic forcings have contributed to the observed warming averaged over available stations. 


\section{Contributions to observed surface temperature change over the period 1951-2010}

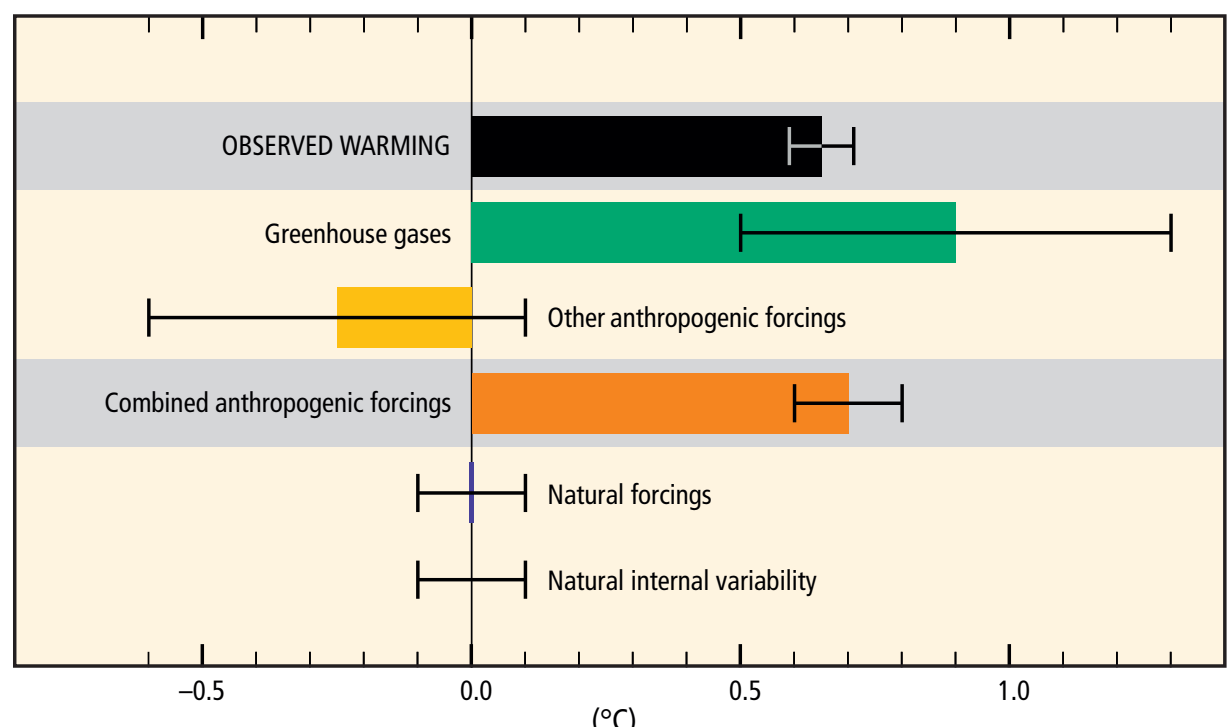

Figure SPM.3 | Assessed likely ranges (whiskers) and their mid-points (bars) for warming trends over the 1951-2010 period from well-mixed greenhouse gases, other anthropogenic forcings (including the cooling effect of aerosols and the effect of land use change), combined anthropogenic forcings, natural forcings and natural internal climate variability (which is the element of climate variability that arises spontaneously within the climate system even in the absence of forcings). The observed surface temperature change is shown in black, with the 5 to $95 \%$ uncertainty range due to observational uncertainty. The attributed warming ranges (colours) are based on observations combined with climate model simulations, in order to estimate the contribution of an individual external forcing to the observed warming. The contribution from the combined anthropogenic forcings can be estimated with less uncertainty than the contributions from greenhouse gases and from other anthropogenic forcings separately. This is because these two contributions partially compensate, resulting in a combined signal that is better constrained by observations. \{Figure 1.9\}

\section{SPM 1.3 Impacts of climate change}

In recent decades, changes in climate have caused impacts on natural and human systems on all continents and across the oceans. Impacts are due to observed climate change, irrespective of its cause, indicating the sensitivity of natural and human systems to changing climate. $\{1.3 .2\}$

Evidence of observed climate change impacts is strongest and most comprehensive for natural systems. In many regions, changing precipitation or melting snow and ice are altering hydrological systems, affecting water resources in terms of quantity and quality (medium confidence). Many terrestrial, freshwater and marine species have shifted their geographic ranges, seasonal activities, migration patterns, abundances and species interactions in response to ongoing climate change (high confidence). Some impacts on human systems have also been attributed to climate change, with a major or minor contribution of climate change distinguishable from other influences (Figure SPM.4). Assessment of many studies covering a wide range of regions and crops shows that negative impacts of climate change on crop yields have been more common than positive impacts (high confidence). Some impacts of ocean acidification on marine organisms have been attributed to human influence (medium confidence). $\{1.3 .2\}$ 
Widespread impacts attributed to climate change based on the available scientific literature since the AR4

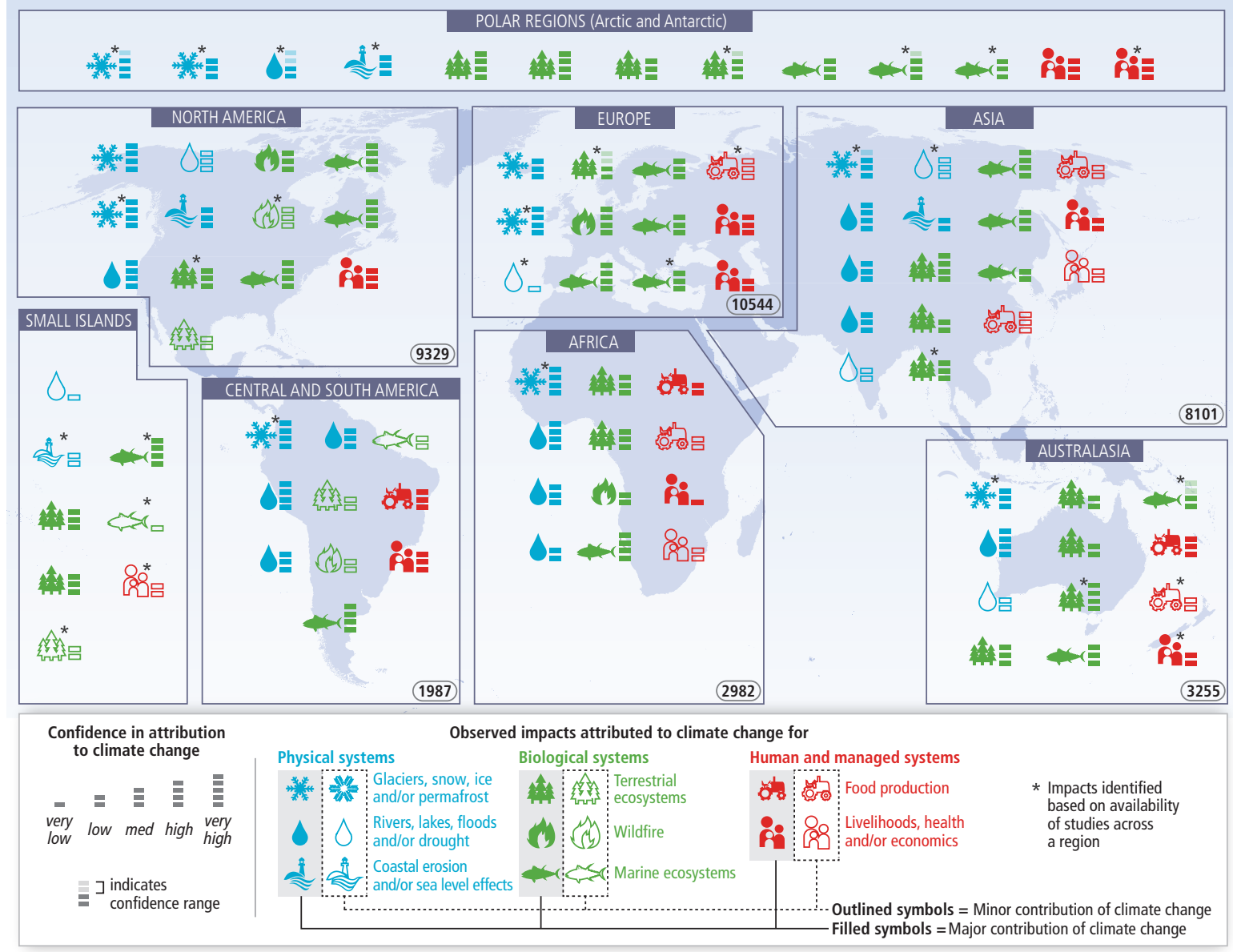

Figure SPM.4 | Based on the available scientific literature since the IPCC Fourth Assessment Report (AR4), there are substantially more impacts in recent decades now attributed to climate change. Attribution requires defined scientific evidence on the role of climate change. Absence from the map of additional impacts attributed to climate change does not imply that such impacts have not occurred. The publications supporting attributed impacts reflect a growing knowledge base, but publications are still limited for many regions, systems and processes, highlighting gaps in data and studies. Symbols indicate categories of attributed impacts, the relative contribution of climate change (major or minor) to the observed impact and confidence in attribution. Each symbol refers to one or more entries in WGII Table SPM.A1, grouping related regional-scale impacts. Numbers in ovals indicate regional totals of climate change publications from 2001 to 2010, based on the Scopus bibliographic database for publications in English with individual countries mentioned in title, abstract or key words (as of July 2011). These numbers provide an overall measure of the available scientific literature on climate change across regions; they do not indicate the number of publications supporting attribution of climate change impacts in each region. Studies for polar regions and small islands are grouped with neighbouring continental regions. The inclusion of publications for assessment of attribution followed IPCC scientific evidence criteria defined in WGII Chapter 18. Publications considered in the attribution analyses come from a broader range of literature assessed in the WGII AR5. See WGII Table SPM.A1 for descriptions of the attributed impacts. \{Figure 1.11\}

\section{SPM $1.4 \quad$ Extreme events}

Changes in many extreme weather and climate events have been observed since about 1950. Some of these changes have been linked to human influences, including a decrease in cold temperature extremes, an increase in warm temperature extremes, an increase in extreme high sea levels and an increase in the number of heavy precipitation events in a number of regions. $\{1.4\}$

It is very likely that the number of cold days and nights has decreased and the number of warm days and nights has increased on the global scale. It is likely that the frequency of heat waves has increased in large parts of Europe, Asia and Australia. It is 
very likely that human influence has contributed to the observed global scale changes in the frequency and intensity of daily temperature extremes since the mid-20th century. It is likely that human influence has more than doubled the probability of occurrence of heat waves in some locations. There is medium confidence that the observed warming has increased heat-related human mortality and decreased cold-related human mortality in some regions. $\{1.4\}$

There are likely more land regions where the number of heavy precipitation events has increased than where it has decreased. Recent detection of increasing trends in extreme precipitation and discharge in some catchments implies greater risks of flooding at regional scale (medium confidence). It is likely that extreme sea levels (for example, as experienced in storm surges) have increased since 1970, being mainly a result of rising mean sea level. $\{1.4\}$

Impacts from recent climate-related extremes, such as heat waves, droughts, floods, cyclones and wildfires, reveal significant vulnerability and exposure of some ecosystems and many human systems to current climate variability (very high confidence). $\{1.4\}$

\section{SPM 2. Future Climate Changes, Risks and Impacts}

Continued emission of greenhouse gases will cause further warming and long-lasting changes in all components of the climate system, increasing the likelihood of severe, pervasive and irreversible impacts for people and ecosystems. Limiting climate change would require substantial and sustained reductions in greenhouse gas emissions which, together with adaptation, can limit climate change risks. $\{2\}$

\section{SPM 2.1 Key drivers of future climate}

Cumulative emissions of $\mathrm{CO}_{2}$ largely determine global mean surface warming by the late 21 st century and beyond. Projections of greenhouse gas emissions vary over a wide range, depending on both socio-economic development and climate policy. $\{2.1\}$

Anthropogenic GHG emissions are mainly driven by population size, economic activity, lifestyle, energy use, land use patterns, technology and climate policy. The Representative Concentration Pathways (RCPs), which are used for making projections based on these factors, describe four different 21st century pathways of GHG emissions and atmospheric concentrations, air pollutant emissions and land use. The RCPs include a stringent mitigation scenario (RCP2.6), two intermediate scenarios (RCP4.5 and RCP6.0) and one scenario with very high GHG emissions (RCP8.5). Scenarios without additional efforts to constrain emissions ('baseline scenarios') lead to pathways ranging between RCP6.0 and RCP8.5 (Figure SPM.5a). RCP2.6 is representative of a scenario that aims to keep global warming likely below $2^{\circ} \mathrm{C}$ above pre-industrial temperatures. The RCPs are consistent with the wide range of scenarios in the literature as assessed by WGIII ${ }^{5}$. $\{2.1, B 0 x 2.2,4.3\}$

Multiple lines of evidence indicate a strong, consistent, almost linear relationship between cumulative $\mathrm{CO}_{2}$ emissions and projected global temperature change to the year 2100 in both the RCPs and the wider set of mitigation scenarios analysed in WGIII (Figure SPM.5b). Any given level of warming is associated with a range of cumulative $\mathrm{CO}_{2}$ emissions ${ }^{6}$, and therefore, e.g., higher emissions in earlier decades imply lower emissions later. \{2.2.5, Table 2.2\}

\footnotetext{
5 Roughly 300 baseline scenarios and 900 mitigation scenarios are categorized by $\mathrm{CO}_{2}$-equivalent concentration $\left(\mathrm{CO}_{2}\right.$-eq) by 2100. The $\mathrm{CO}_{2}$-eq includes the forcing due to all GHGs (including halogenated gases and tropospheric ozone), aerosols and albedo change.

6 Quantification of this range of $\mathrm{CO}_{2}$ emissions requires taking into account non- $\mathrm{CO}_{2}$ drivers.
} 

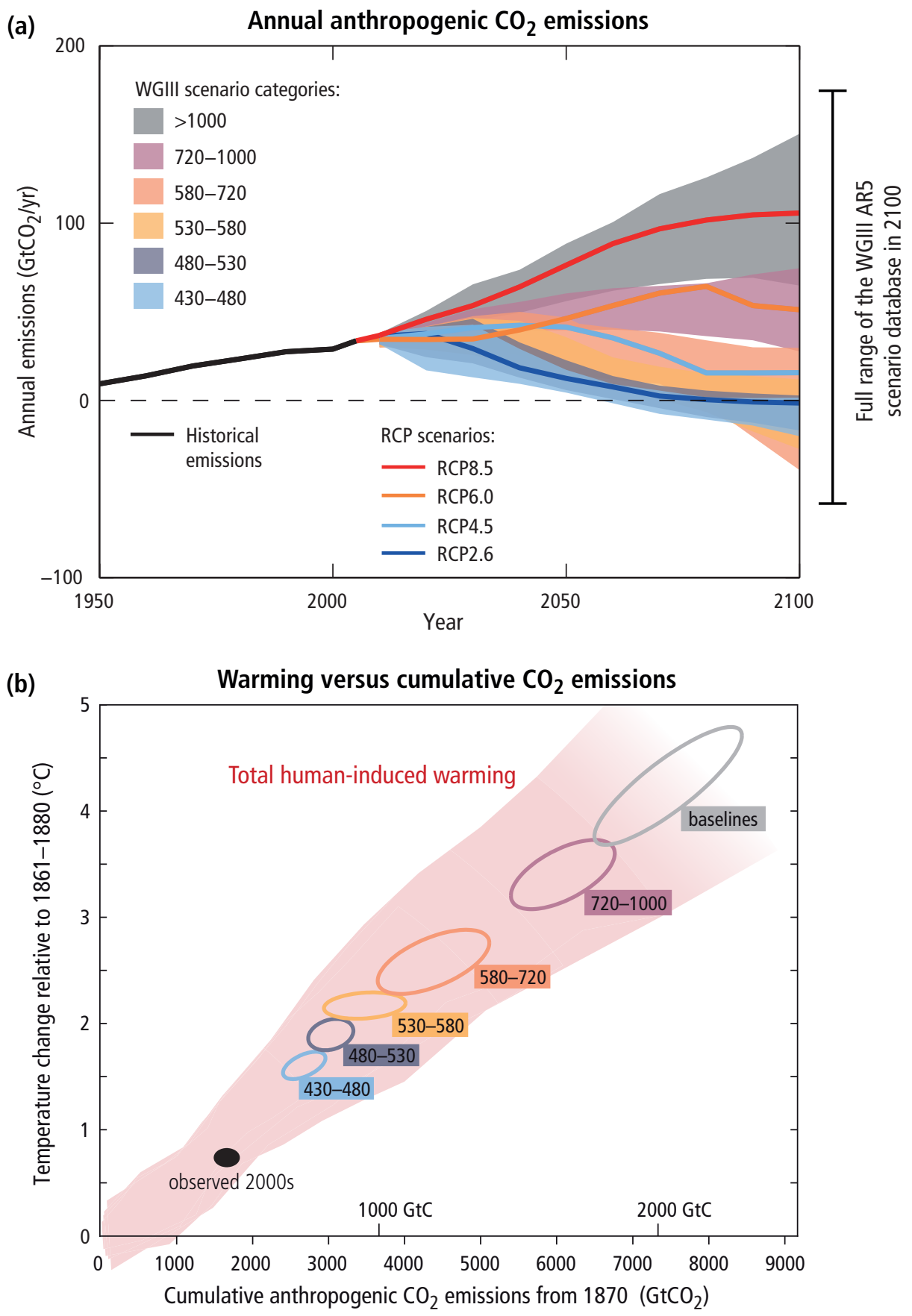

Figure SPM.5 | (a) Emissions of carbon dioxide $\left(\mathrm{CO}_{2}\right)$ alone in the Representative Concentration Pathways (RCPs) (lines) and the associated scenario categories used in WGIII (coloured areas show 5 to 95\% range). The WGIII scenario categories summarize the wide range of emission scenarios published in the scientific literature and are defined on the basis of $\mathrm{CO}_{2}$-eq concentration levels (in ppm) in 2100. The time series of other greenhouse gas emissions are shown in Box 2.2, Figure 1. (b) Global mean surface temperature increase at the time global $\mathrm{CO}_{2}$ emissions reach a given net cumulative total, plotted as a function of that total, from various lines of evidence. Coloured plume shows the spread of past and future projections from a hierarchy of climatecarbon cycle models driven by historical emissions and the four RCPs over all times out to 2100, and fades with the decreasing number of available models. Ellipses show total anthropogenic warming in 2100 versus cumulative $\mathrm{CO}_{2}$ emissions from 1870 to 2100 from a simple climate model (median climate response) under the scenario categories used in WGIII. The width of the ellipses in terms of temperature is caused by the impact of different scenarios for non- $\mathrm{CO}_{2}$ climate drivers. The filled black ellipse shows observed emissions to 2005 and observed temperatures in the decade 2000-2009 with associated uncertainties. \{Box 2.2, Figure 1; Figure 2.3\} 
Multi-model results show that limiting total human-induced warming to less than $2^{\circ} \mathrm{C}$ relative to the period $1861-1880$ with a probability of $>66 \%{ }^{7}$ would require cumulative $\mathrm{CO}_{2}$ emissions from all anthropogenic sources since 1870 to remain below about $2900 \mathrm{GtCO}_{2}$ (with a range of 2550 to $3150 \mathrm{GtCO}_{2}$ depending on non- $\mathrm{CO}_{2}$ drivers). About $1900 \mathrm{GtCO}_{2}{ }^{8}$ had already been emitted by 2011. For additional context see Table 2.2. $\{2.2 .5\}$

\section{SPM 2.2 Projected changes in the climate system}

Surface temperature is projected to rise over the 21 st century under all assessed emission scenarios. It is very likely that heat waves will occur more often and last longer, and that extreme precipitation events will become more intense and frequent in many regions. The ocean will continue to warm and acidify, and global mean sea level to rise. $\{2.2\}$

The projected changes in Section SPM 2.2 are for 2081-2100 relative to 1986-2005, unless otherwise indicated.

Future climate will depend on committed warming caused by past anthropogenic emissions, as well as future anthropogenic emissions and natural climate variability. The global mean surface temperature change for the period 2016-2035 relative to $1986-2005$ is similar for the four RCPs and will likely be in the range $0.3^{\circ} \mathrm{C}$ to $0.7^{\circ} \mathrm{C}$ (medium confidence). This assumes that there will be no major volcanic eruptions or changes in some natural sources (e.g., $\mathrm{CH}_{4}$ and $\mathrm{N}_{2} \mathrm{O}$ ), or unexpected changes in total solar irradiance. By mid-21st century, the magnitude of the projected climate change is substantially affected by the choice of emissions scenario. \{2.2.1, Table 2.1\}

Relative to 1850-1900, global surface temperature change for the end of the 21st century (2081-2100) is projected to likely exceed $1.5^{\circ} \mathrm{C}$ for RCP4.5, RCP6.0 and RCP8.5 (high confidence). Warming is likely to exceed $2^{\circ} \mathrm{C}$ for RCP6.0 and RCP8.5 (high confidence), more likely than not to exceed $2^{\circ} \mathrm{C}$ for RCP4.5 (medium confidence), but unlikely to exceed $2^{\circ} \mathrm{C}$ for RCP2.6 (medium confidence). \{2.2.1\}

The increase of global mean surface temperature by the end of the 21st century (2081-2100) relative to 1986-2005 is likely to be $0.3^{\circ} \mathrm{C}$ to $1.7^{\circ} \mathrm{C}$ under $\mathrm{RCP} 2.6,1.1^{\circ} \mathrm{C}$ to $2.6^{\circ} \mathrm{C}$ under $\mathrm{RCP} 4.5,1.4^{\circ} \mathrm{C}$ to $3.1^{\circ} \mathrm{C}$ under $\mathrm{RCP} 6.0$ and $2.6^{\circ} \mathrm{C}$ to $4.8^{\circ} \mathrm{C}$ under RCP8.5 ${ }^{9}$. The Arctic region will continue to warm more rapidly than the global mean (Figure SPM.6a, Figure SPM.7a). \{2.2.1, Figure 2.1, Figure 2.2, Table 2.1\}

It is virtually certain that there will be more frequent hot and fewer cold temperature extremes over most land areas on daily and seasonal timescales, as global mean surface temperature increases. It is very likely that heat waves will occur with a higher frequency and longer duration. Occasional cold winter extremes will continue to occur. \{2.2.1\}

7 Corresponding figures for limiting warming to $2^{\circ} \mathrm{C}$ with a probability of $>50 \%$ and $>33 \%$ are $3000 \mathrm{GtCO}_{2}$ (range of 2900 to $3200 \mathrm{GtCO}_{2}$ ) and $3300 \mathrm{GtCO}_{2}$ (range of 2950 to $3800 \mathrm{GtCO}_{2}$ ) respectively. Higher or lower temperature limits would imply larger or lower cumulative emissions respectively.

8 This corresponds to about two thirds of the $2900 \mathrm{GtCO}_{2}$ that would limit warming to less than $2^{\circ} \mathrm{C}$ with a probability of $>66 \%$; to about $63 \%$ of the total amount of $3000 \mathrm{GtCO}_{2}$ that would limit warming to less than $2^{\circ} \mathrm{C}$ with a probability of $>50 \%$; and to about $58 \%$ of the total amount of $3300 \mathrm{GtCO}_{2}$ that would limit warming to less than $2^{\circ} \mathrm{C}$ with a probability of $>33 \%$.

9 The period $1986-2005$ is approximately $0.61[0.55 \text { to } 0.67]^{\circ} \mathrm{C}$ warmer than $1850-1900 .\{2.2 .1\}$ 
(a)

Global average surface temperature change

(relative to 1986-2005)

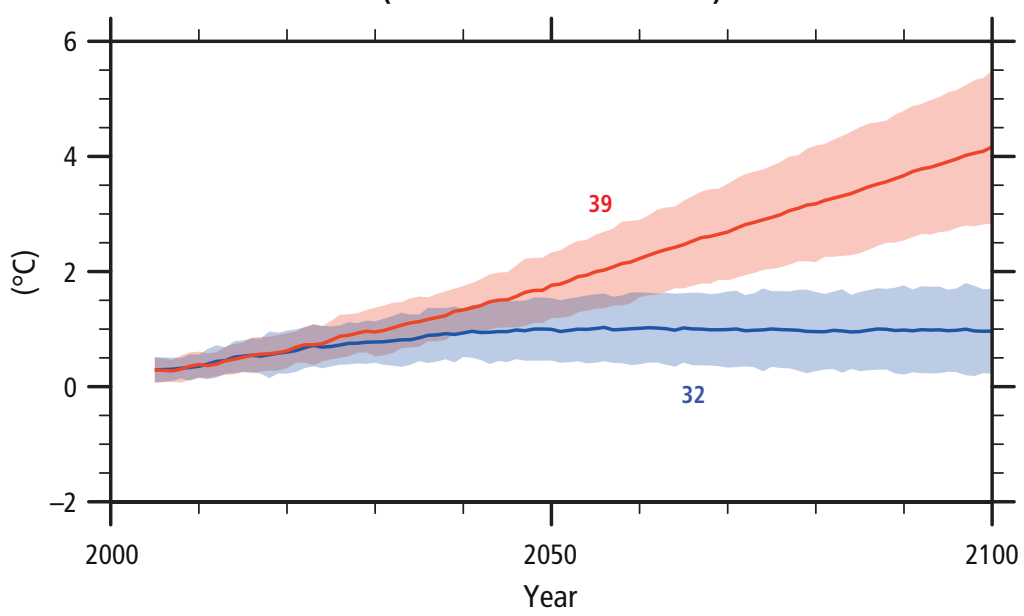

(b)

Global mean sea level rise

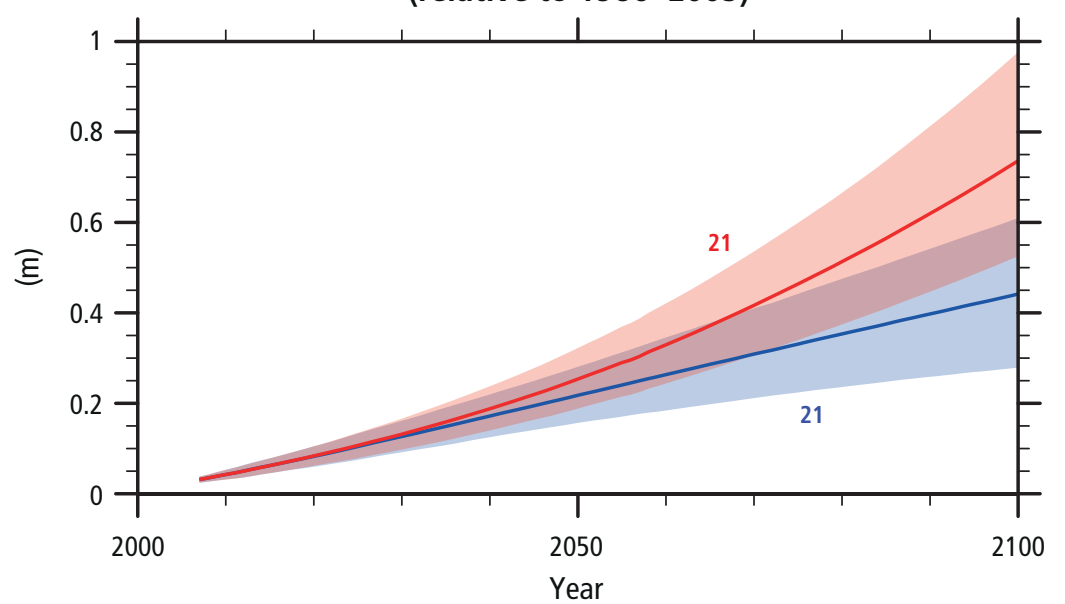

Mean over

$2081-2100$

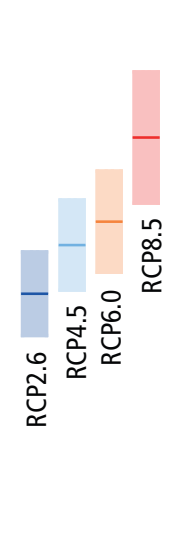

Mean over

$2081-2100$

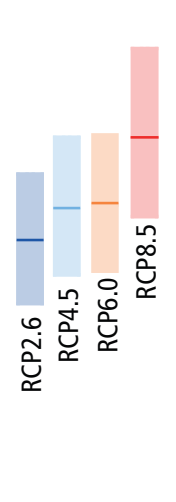

Figure SPM.6 | Global average surface temperature change (a) and global mean sea level rise ${ }^{10}$ (b) from 2006 to 2100 as determined by multi-model simulations. All changes are relative to 1986-2005. Time series of projections and a measure of uncertainty (shading) are shown for scenarios RCP2.6 (blue) and RCP8.5 (red). The mean and associated uncertainties averaged over 2081-2100 are given for all RCP scenarios as coloured vertical bars at the right hand side of each panel. The number of Coupled Model Intercomparison Project Phase 5 (CMIP5) models used to calculate the multi-model mean is indicated. $\{2.2$, Figure 2.1$\}$

Changes in precipitation will not be uniform. The high latitudes and the equatorial Pacific are likely to experience an increase in annual mean precipitation under the RCP8.5 scenario. In many mid-latitude and subtropical dry regions, mean precipitation will likely decrease, while in many mid-latitude wet regions, mean precipitation will likely increase under the RCP8.5 scenario (Figure SPM.7b). Extreme precipitation events over most of the mid-latitude land masses and over wet tropical regions will very likely become more intense and more frequent. $\{2.2 .2$, Figure 2.2$\}$

The global ocean will continue to warm during the 21st century, with the strongest warming projected for the surface in tropical and Northern Hemisphere subtropical regions (Figure SPM.7a). \{2.2.3, Figure 2.2\}

10 Based on current understanding (from observations, physical understanding and modelling), only the collapse of marine-based sectors of the Antarctic ice sheet, if initiated, could cause global mean sea level to rise substantially above the likely range during the 21st century. There is medium confidence that this additional contribution would not exceed several tenths of a meter of sea level rise during the 21st century. 
RCP2.6

RCP8.5

(a)

Change in average surface temperature (1986-2005 to 2081-2100)

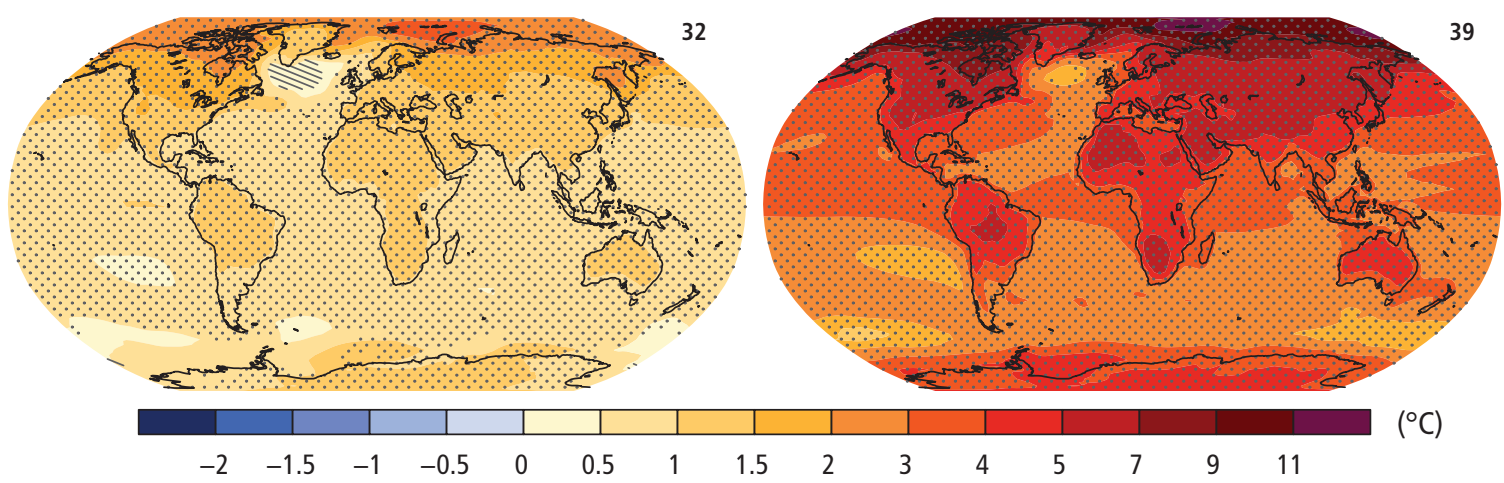

(b)

Change in average precipitation (1986-2005 to 2081-2100)

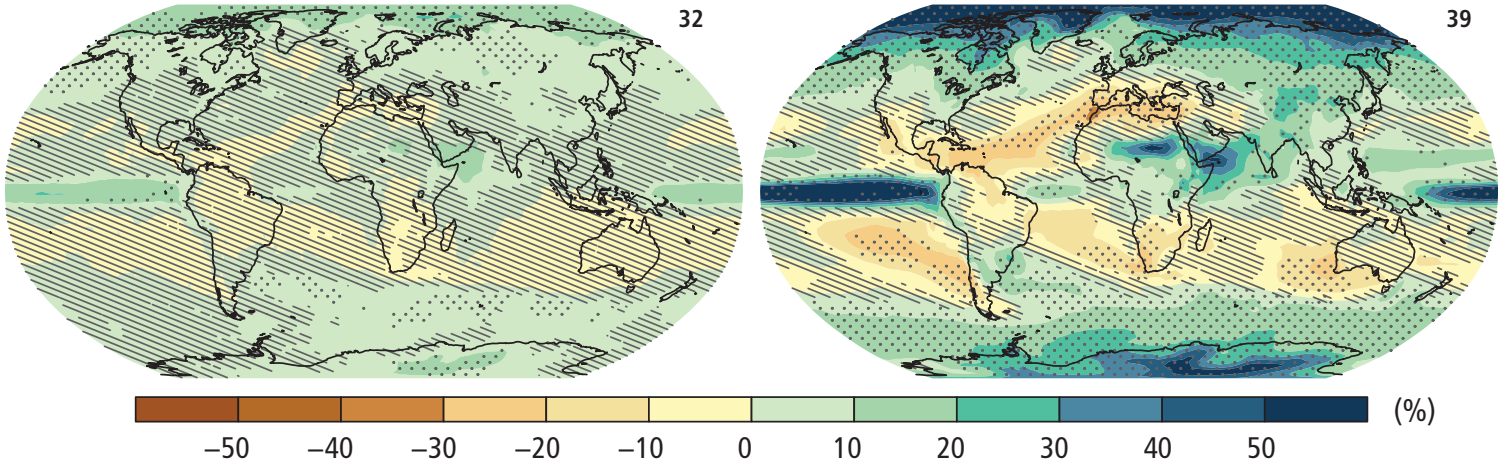

Figure SPM.7 | Change in average surface temperature (a) and change in average precipitation (b) based on multi-model mean projections for 2081-2100 relative to 1986-2005 under the RCP2.6 (left) and RCP8.5 (right) scenarios. The number of models used to calculate the multi-model mean is indicated in the upper right corner of each panel. Stippling (i.e., dots) shows regions where the projected change is large compared to natural internal variability and where at least $90 \%$ of models agree on the sign of change. Hatching (i.e., diagonal lines) shows regions where the projected change is less than one standard deviation of the natural internal variability. \{2.2, Figure 2.2$\}$

Earth System Models project a global increase in ocean acidification for all RCP scenarios by the end of the 21st century, with a slow recovery after mid-century under RCP2.6. The decrease in surface ocean pH is in the range of 0.06 to 0.07 (15 to 17\% increase in acidity) for RCP2.6, 0.14 to 0.15 (38 to $41 \%$ ) for RCP4.5, 0.20 to 0.21 (58 to $62 \%$ ) for RCP6.0 and 0.30 to 0.32 (100 to $109 \%$ ) for RCP8.5. \{2.2.4, Figure 2.1$\}$

Year-round reductions in Arctic sea ice are projected for all RCP scenarios. A nearly ice-free ${ }^{11}$ Arctic Ocean in the summer seaice minimum in September before mid-century is likely for RCP8.5' (medium confidence). $\{2.2 .3$, Figure 2.1\}

It is virtually certain that near-surface permafrost extent at high northern latitudes will be reduced as global mean surface temperature increases, with the area of permafrost near the surface (upper $3.5 \mathrm{~m}$ ) projected to decrease by $37 \%$ (RCP2.6) to $81 \%$ (RCP8.5) for the multi-model average (medium confidence). $\{2.2 .3\}$

The global glacier volume, excluding glaciers on the periphery of Antarctica (and excluding the Greenland and Antarctic ice sheets), is projected to decrease by 15 to $55 \%$ for RCP2.6 and by 35 to $85 \%$ for RCP8.5 (medium confidence). \{2.2.3\}

11 When sea-ice extent is less than one million $\mathrm{km}^{2}$ for at least five consecutive years.

12 Based on an assessment of the subset of models that most closely reproduce the climatological mean state and 1979-2012 trend of the Arctic sea-ice extent. 
There has been significant improvement in understanding and projection of sea level change since the AR4. Global mean sea level rise will continue during the 21st century, very likely at a faster rate than observed from 1971 to 2010 . For the period 2081-2100 relative to 1986-2005, the rise will likely be in the ranges of 0.26 to $0.55 \mathrm{~m}$ for RCP2.6, and of 0.45 to $0.82 \mathrm{~m}$ for RCP8.5 (medium confidence) ${ }^{10}$ (Figure SPM.6b). Sea level rise will not be uniform across regions. By the end of the 21 st century, it is very likely that sea level will rise in more than about $95 \%$ of the ocean area. About $70 \%$ of the coastlines worldwide are projected to experience a sea level change within $\pm 20 \%$ of the global mean. $\{2.2 .3\}$

\section{SPM 2.3 Future risks and impacts caused by a changing climate}

Climate change will amplify existing risks and create new risks for natural and human systems. Risks are unevenly distributed and are generally greater for disadvantaged people and communities in countries at all levels of development. $\{2.3\}$

Risk of climate-related impacts results from the interaction of climate-related hazards (including hazardous events and trends) with the vulnerability and exposure of human and natural systems, including their ability to adapt. Rising rates and magnitudes of warming and other changes in the climate system, accompanied by ocean acidification, increase the risk of severe, pervasive and in some cases irreversible detrimental impacts. Some risks are particularly relevant for individual regions (Figure SPM.8), while others are global. The overall risks of future climate change impacts can be reduced by limiting the rate and magnitude of climate change, including ocean acidification. The precise levels of climate change sufficient to trigger abrupt and irreversible change remain uncertain, but the risk associated with crossing such thresholds increases with rising temperature (medium confidence). For risk assessment, it is important to evaluate the widest possible range of impacts, including low-probability outcomes with large consequences. $\{1.5,2.3,2.4,3.3$, Box Introduction.1, Box 2.3, Box 2.4\}

A large fraction of species faces increased extinction risk due to climate change during and beyond the 21st century, especially as climate change interacts with other stressors (high confidence). Most plant species cannot naturally shift their geographical ranges sufficiently fast to keep up with current and high projected rates of climate change in most landscapes; most small mammals and freshwater molluscs will not be able to keep up at the rates projected under RCP4.5 and above in flat landscapes in this century (high confidence). Future risk is indicated to be high by the observation that natural global climate change at rates lower than current anthropogenic climate change caused significant ecosystem shifts and species extinctions during the past millions of years. Marine organisms will face progressively lower oxygen levels and high rates and magnitudes of ocean acidification (high confidence), with associated risks exacerbated by rising ocean temperature extremes (medium confidence). Coral reefs and polar ecosystems are highly vulnerable. Coastal systems and low-lying areas are at risk from sea level rise, which will continue for centuries even if the global mean temperature is stabilized (high confidence). $\{2.3,2.4$, Figure 2.5$\}$

Climate change is projected to undermine food security (Figure SPM.9). Due to projected climate change by the mid-21st century and beyond, global marine species redistribution and marine biodiversity reduction in sensitive regions will challenge the sustained provision of fisheries productivity and other ecosystem services (high confidence). For wheat, rice and maize in tropical and temperate regions, climate change without adaptation is projected to negatively impact production for local temperature increases of $2^{\circ} \mathrm{C}$ or more above late 20th century levels, although individual locations may benefit (medium confidence). Global temperature increases of $\sim 4^{\circ} \mathrm{C}$ or more ${ }^{13}$ above late 20th century levels, combined with increasing food demand, would pose large risks to food security globally (high confidence). Climate change is projected to reduce renewable surface water and groundwater resources in most dry subtropical regions (robust evidence, high agreement), intensifying competition for water among sectors (limited evidence, medium agreement). $\{2.3 .1,2.3 .2\}$

13 Projected warming averaged over land is larger than global average warming for all RCP scenarios for the period 2081-2100 relative to $1986-2005$. For regional projections, see Figure SPM.7. \{2.2\} 


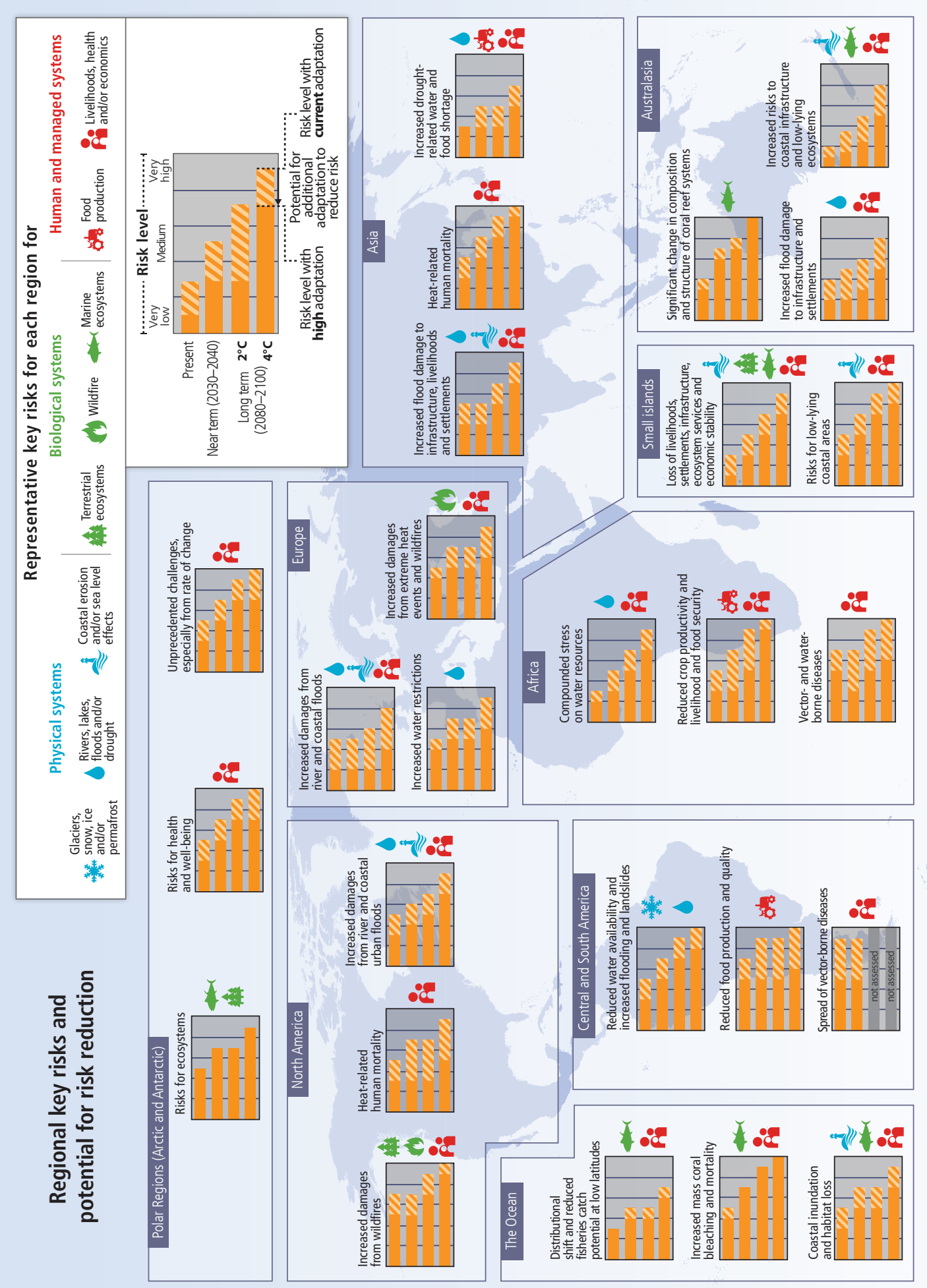

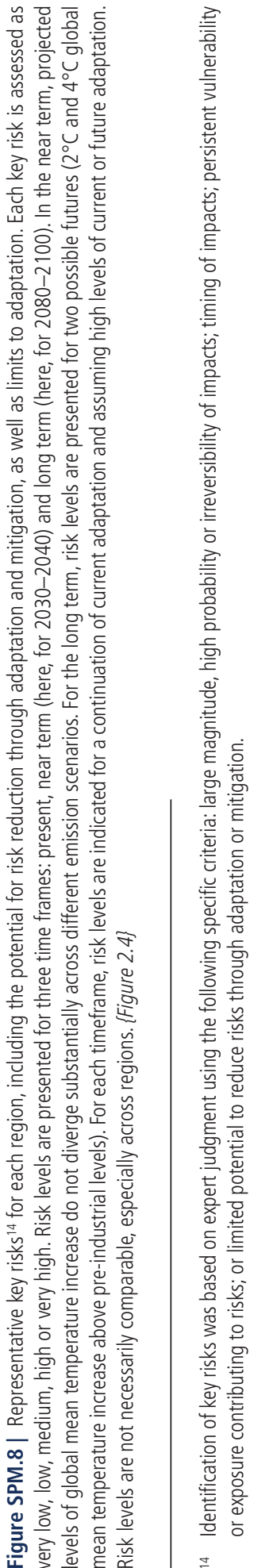


(a)

\section{Climate change poses risks for food production}

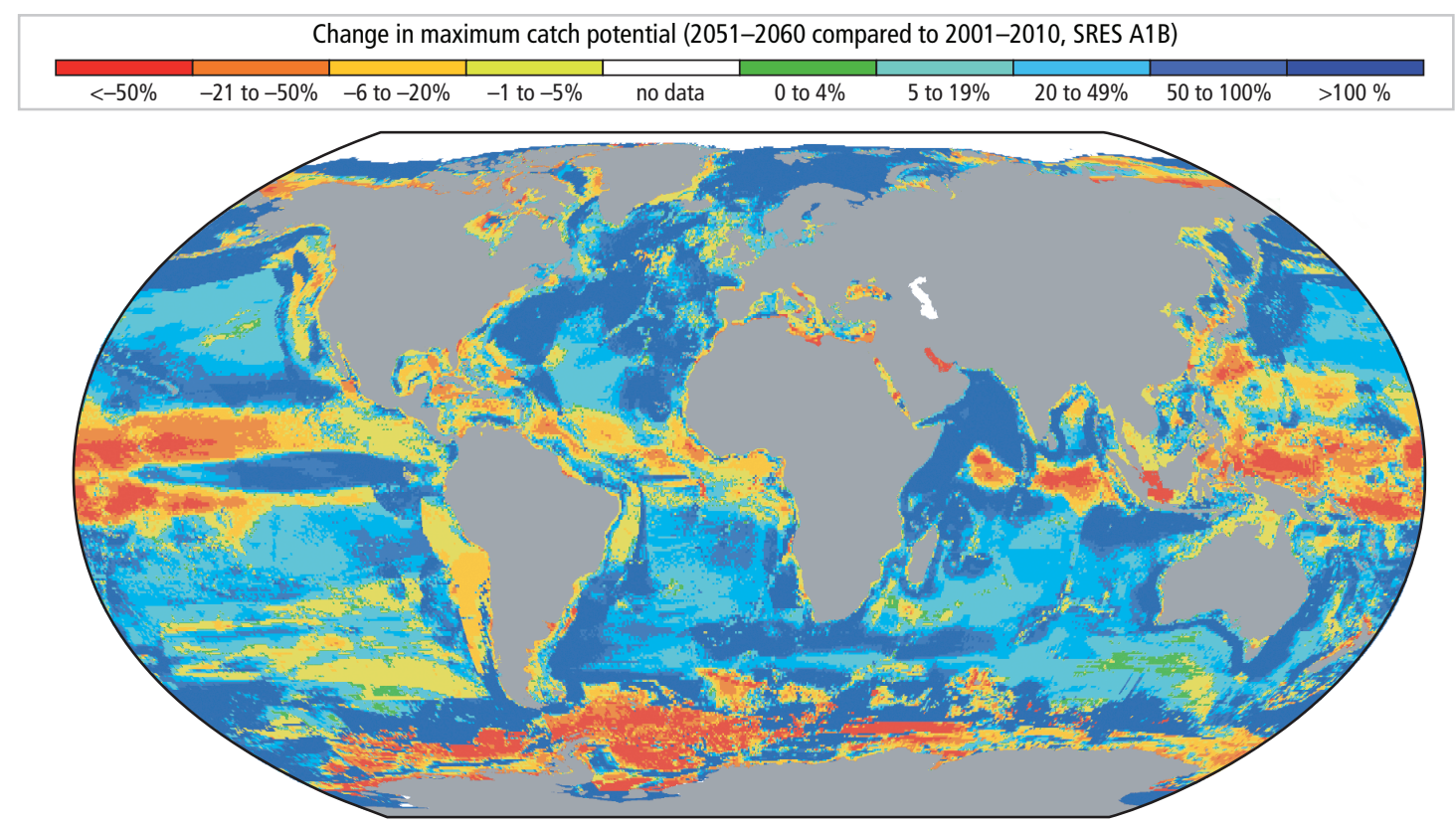

(b)

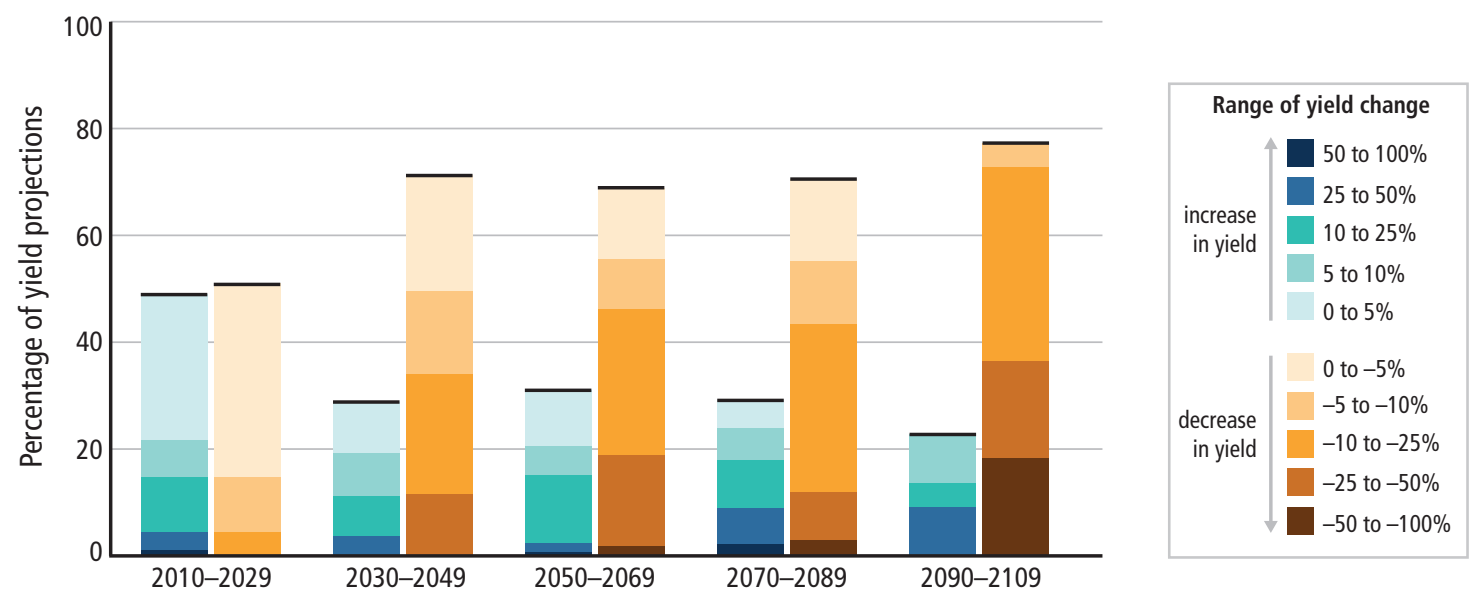

Figure SPM.9 | (a) Projected global redistribution of maximum catch potential of $~ 1000$ exploited marine fish and invertebrate species. Projections compare the 10-year averages 2001-2010 and 2051-2060 using ocean conditions based on a single climate model under a moderate to high warming scenario, without analysis of potential impacts of overfishing or ocean acidification. (b) Summary of projected changes in crop yields (mostly wheat, maize, rice and soy), due to climate change over the 21 st century. Data for each timeframe sum to $100 \%$, indicating the percentage of projections showing yield increases versus decreases. The figure includes projections (based on 1090 data points) for different emission scenarios, for tropical and temperate regions and for adaptation and no-adaptation cases combined. Changes in crop yields are relative to late 20th century levels. \{Figure 2.6a, Figure 2.7\}

Until mid-century, projected climate change will impact human health mainly by exacerbating health problems that already exist (very high confidence). Throughout the 21 st century, climate change is expected to lead to increases in ill-health in many regions and especially in developing countries with low income, as compared to a baseline without climate change (high confidence). By 2100 for RCP8.5, the combination of high temperature and humidity in some areas for parts of the year is expected to compromise common human activities, including growing food and working outdoors (high confidence). \{2.3.2\}

In urban areas climate change is projected to increase risks for people, assets, economies and ecosystems, including risks from heat stress, storms and extreme precipitation, inland and coastal flooding, landslides, air pollution, drought, water scarcity, sea level rise and storm surges (very high confidence). These risks are amplified for those lacking essential infrastructure and services or living in exposed areas. \{2.3.2\} 
Rural areas are expected to experience major impacts on water availability and supply, food security, infrastructure and agricultural incomes, including shifts in the production areas of food and non-food crops around the world (high confidence). $\{2.3 .2\}$

Aggregate economic losses accelerate with increasing temperature (limited evidence, high agreement), but global economic impacts from climate change are currently difficult to estimate. From a poverty perspective, climate change impacts are projected to slow down economic growth, make poverty reduction more difficult, further erode food security and prolong existing and create new poverty traps, the latter particularly in urban areas and emerging hotspots of hunger (medium confidence). International dimensions such as trade and relations among states are also important for understanding the risks of climate change at regional scales. $\{2.3 .2\}$

Climate change is projected to increase displacement of people (medium evidence, high agreement). Populations that lack the resources for planned migration experience higher exposure to extreme weather events, particularly in developing countries with low income. Climate change can indirectly increase risks of violent conflicts by amplifying well-documented drivers of these conflicts such as poverty and economic shocks (medium confidence). $\{2.3 .2\}$

\section{SPM 2.4 Climate change beyond 2100, irreversibility and abrupt changes}

Many aspects of climate change and associated impacts will continue for centuries, even if anthropogenic emissions of greenhouse gases are stopped. The risks of abrupt or irreversible changes increase as the magnitude of the warming increases. $\{2.4\}$

Warming will continue beyond 2100 under all RCP scenarios except RCP2.6. Surface temperatures will remain approximately constant at elevated levels for many centuries after a complete cessation of net anthropogenic $\mathrm{CO}_{2}$ emissions. A large fraction of anthropogenic climate change resulting from $\mathrm{CO}_{2}$ emissions is irreversible on a multi-century to millennial timescale, except in the case of a large net removal of $\mathrm{CO}_{2}$ from the atmosphere over a sustained period. $\{2.4$, Figure 2.8\}

Stabilization of global average surface temperature does not imply stabilization for all aspects of the climate system. Shifting biomes, soil carbon, ice sheets, ocean temperatures and associated sea level rise all have their own intrinsic long timescales which will result in changes lasting hundreds to thousands of years after global surface temperature is stabilized. $\{2.1,2.4\}$

There is high confidence that ocean acidification will increase for centuries if $\mathrm{CO}_{2}$ emissions continue, and will strongly affect marine ecosystems. $\{2.4\}$

It is virtually certain that global mean sea level rise will continue for many centuries beyond 2100, with the amount of rise dependent on future emissions. The threshold for the loss of the Greenland ice sheet over a millennium or more, and an associated sea level rise of up to $7 \mathrm{~m}$, is greater than about $1^{\circ} \mathrm{C}$ (low confidence) but less than about $4^{\circ} \mathrm{C}$ (medium confidence) of global warming with respect to pre-industrial temperatures. Abrupt and irreversible ice loss from the Antarctic ice sheet is possible, but current evidence and understanding is insufficient to make a quantitative assessment. $\{2.4\}$

Magnitudes and rates of climate change associated with medium- to high-emission scenarios pose an increased risk of abrupt and irreversible regional-scale change in the composition, structure and function of marine, terrestrial and freshwater ecosystems, including wetlands (medium confidence). A reduction in permafrost extent is virtually certain with continued rise in global temperatures. $\{2.4\}$ 


\section{SPM 3. Future Pathways for Adaptation, Mitigation and Sustainable Development}

Adaptation and mitigation are complementary strategies for reducing and managing the risks of climate change. Substantial emissions reductions over the next few decades can reduce climate risks in the 21 st century and beyond, increase prospects for effective adaptation, reduce the costs and challenges of mitigation in the longer term and contribute to climate-resilient pathways for sustainable development. $\{3.2,3.3,3.4\}$

\section{SPM 3.1 Foundations of decision-making about climate change}

Effective decision-making to limit climate change and its effects can be informed by a wide range of analytical approaches for evaluating expected risks and benefits, recognizing the importance of governance, ethical dimensions, equity, value judgments, economic assessments and diverse perceptions and responses to risk and uncertainty. \{3.1\}

Sustainable development and equity provide a basis for assessing climate policies. Limiting the effects of climate change is necessary to achieve sustainable development and equity, including poverty eradication. Countries' past and future contributions to the accumulation of GHGs in the atmosphere are different, and countries also face varying challenges and circumstances and have different capacities to address mitigation and adaptation. Mitigation and adaptation raise issues of equity, justice and fairness. Many of those most vulnerable to climate change have contributed and contribute little to GHG emissions. Delaying mitigation shifts burdens from the present to the future, and insufficient adaptation responses to emerging impacts are already eroding the basis for sustainable development. Comprehensive strategies in response to climate change that are consistent with sustainable development take into account the co-benefits, adverse side effects and risks that may arise from both adaptation and mitigation options. \{3.1, 3.5, Box 3.4\}

The design of climate policy is influenced by how individuals and organizations perceive risks and uncertainties and take them into account. Methods of valuation from economic, social and ethical analysis are available to assist decision-making. These methods can take account of a wide range of possible impacts, including low-probability outcomes with large consequences. But they cannot identify a single best balance between mitigation, adaptation and residual climate impacts. \{3.1\}

Climate change has the characteristics of a collective action problem at the global scale, because most GHGs accumulate over time and mix globally, and emissions by any agent (e.g., individual, community, company, country) affect other agents. Effective mitigation will not be achieved if individual agents advance their own interests independently. Cooperative responses, including international cooperation, are therefore required to effectively mitigate GHG emissions and address other climate change issues. The effectiveness of adaptation can be enhanced through complementary actions across levels, including international cooperation. The evidence suggests that outcomes seen as equitable can lead to more effective cooperation. $\{3.1\}$

\section{SPM 3.2 Climate change risks reduced by mitigation and adaptation}

Without additional mitigation efforts beyond those in place today, and even with adaptation, warming by the end of the 21st century will lead to high to very high risk of severe, widespread and irreversible impacts globally (high confidence). Mitigation involves some level of co-benefits and of risks due to adverse side effects, but these risks do not involve the same possibility of severe, widespread and irreversible impacts as risks from climate change, increasing the benefits from near-term mitigation efforts. $\{3.2,3.4\}$

Mitigation and adaptation are complementary approaches for reducing risks of climate change impacts over different timescales (high confidence). Mitigation, in the near term and through the century, can substantially reduce climate change 
impacts in the latter decades of the 21 st century and beyond. Benefits from adaptation can already be realized in addressing current risks, and can be realized in the future for addressing emerging risks. $\{3.2,4.5\}$

Five Reasons For Concern (RFCs) aggregate climate change risks and illustrate the implications of warming and of adaptation limits for people, economies and ecosystems across sectors and regions. The five RFCs are associated with: (1) Unique and threatened systems, (2) Extreme weather events, (3) Distribution of impacts, (4) Global aggregate impacts, and (5) Largescale singular events. In this report, the RFCs provide information relevant to Article 2 of UNFCCC. \{Box 2.4\}

Without additional mitigation efforts beyond those in place today, and even with adaptation, warming by the end of the 21st century will lead to high to very high risk of severe, widespread and irreversible impacts globally (high confidence) (Figure SPM.10). In most scenarios without additional mitigation efforts (those with 2100 atmospheric concentrations

(a) Risks from climate change...

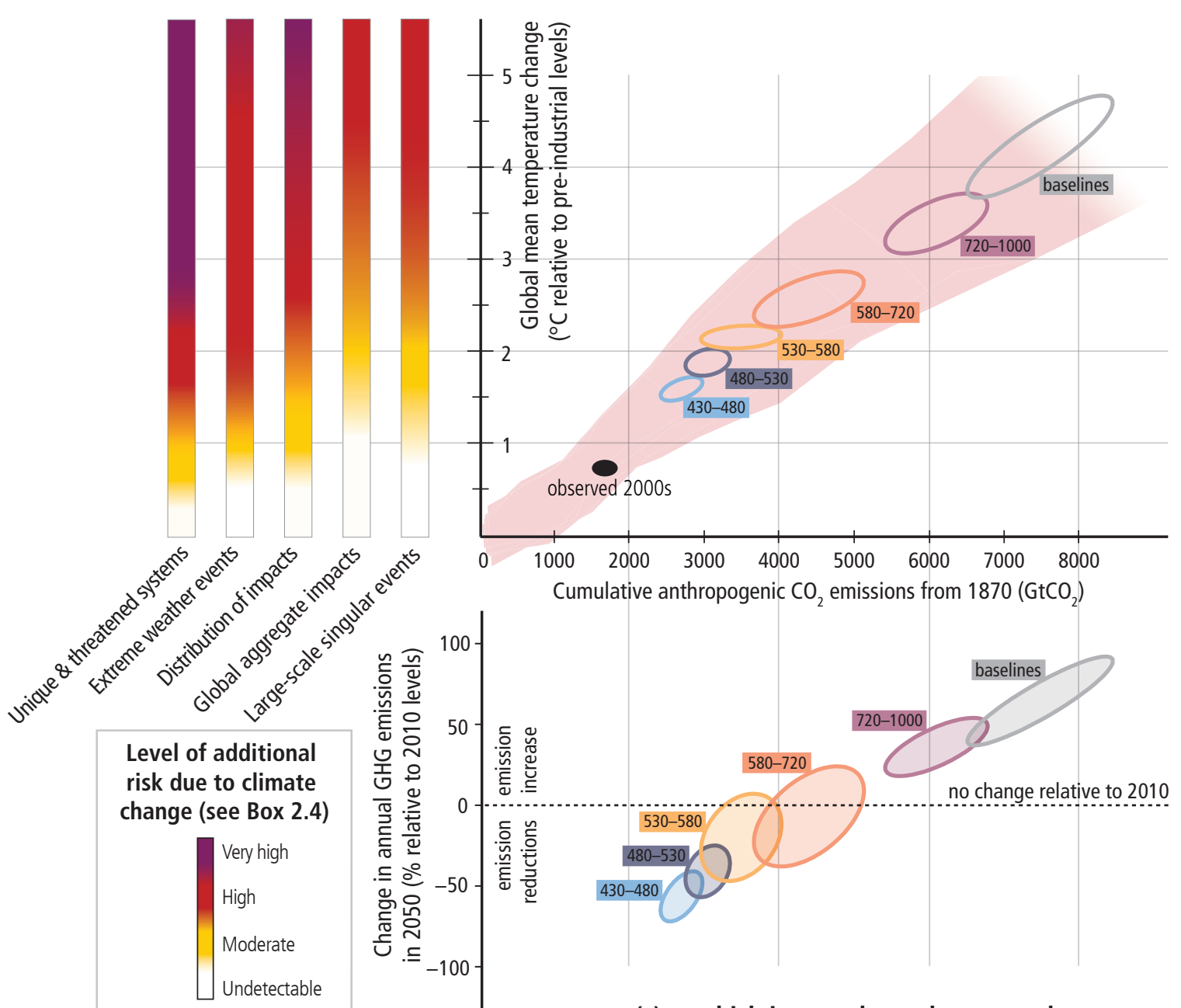

(c) ... which in turn depend on annual

GHG emissions over the next decades

Figure SPM.10 The relationship between risks from climate change, temperature change, cumulative carbon dioxide $\left(\mathrm{CO}_{2}\right)$ emissions and changes in annual greenhouse gas (GHG) emissions by 2050. Limiting risks across Reasons For Concern (a) would imply a limit for cumulative emissions of $\mathrm{CO}_{2}$ (b) which would constrain annual GHG emissions over the next few decades (c). Panel a reproduces the five Reasons For Concern \{Box 2.4\}. Panel b links temperature changes to cumulative $\mathrm{CO}_{2}$ emissions (in $\mathrm{GtCO}_{2}$ ) from 1870. They are based on Coupled Model Intercomparison Project Phase 5 (CMIP5) simulations (pink plume) and on a simple climate model (median climate response in 2100), for the baselines and five mitigation scenario categories (six ellipses). Details are provided in Figure SPM.5. Panel c shows the relationship between the cumulative $\mathrm{CO}_{2}$ emissions (in $\mathrm{GtCO}_{2}$ ) of the scenario categories and their associated change in annual GHG emissions by 2050, expressed in percentage change (in percent $\mathrm{GtCO}_{2}$-eq per year) relative to 2010. The ellipses correspond to the same scenario categories as in Panel b, and are built with a similar method (see details in Figure SPM.5). \{Figure 3.1\} 
$>1000$ ppm CO $\mathrm{CO}_{2}$-eq), warming is more likely than not to exceed $4^{\circ} \mathrm{C}$ above pre-industrial levels by 2100 (Table SPM.1). The risks associated with temperatures at or above $4^{\circ} \mathrm{C}$ include substantial species extinction, global and regional food insecurity, consequential constraints on common human activities and limited potential for adaptation in some cases (high confidence). Some risks of climate change, such as risks to unique and threatened systems and risks associated with extreme weather events, are moderate to high at temperatures $1^{\circ} \mathrm{C}$ to $2^{\circ} \mathrm{C}$ above pre-industrial levels. $\{2.3$, Figure 2.5, 3.2, 3.4, Box 2.4, Table SPM.1\}

Substantial cuts in GHG emissions over the next few decades can substantially reduce risks of climate change by limiting warming in the second half of the 21 st century and beyond. Cumulative emissions of $\mathrm{CO}_{2}$ largely determine global mean surface warming by the late 21 st century and beyond. Limiting risks across RFCs would imply a limit for cumulative emissions of $\mathrm{CO}_{2}$. Such a limit would require that global net emissions of $\mathrm{CO}_{2}$ eventually decrease to zero and would constrain annual emissions over the next few decades (Figure SPM.10) (high confidence). But some risks from climate damages are unavoidable, even with mitigation and adaptation. $\{2.2 .5,3.2,3.4\}$

Mitigation involves some level of co-benefits and risks, but these risks do not involve the same possibility of severe, widespread and irreversible impacts as risks from climate change. Inertia in the economic and climate system and the possibility of irreversible impacts from climate change increase the benefits from near-term mitigation efforts (high confidence). Delays in additional mitigation or constraints on technological options increase the longer-term mitigation costs to hold climate change risks at a given level (Table SPM.2). \{3.2, 3.4\}

\section{SPM 3.3 Characteristics of adaptation pathways}

Adaptation can reduce the risks of climate change impacts, but there are limits to its effectiveness, especially with greater magnitudes and rates of climate change. Taking a longerterm perspective, in the context of sustainable development, increases the likelihood that more immediate adaptation actions will also enhance future options and preparedness. \{3.3\}

Adaptation can contribute to the well-being of populations, the security of assets and the maintenance of ecosystem goods, functions and services now and in the future. Adaptation is place- and context-specific (high confidence). A first step towards adaptation to future climate change is reducing vulnerability and exposure to present climate variability (high confidence). Integration of adaptation into planning, including policy design, and decision-making can promote synergies with development and disaster risk reduction. Building adaptive capacity is crucial for effective selection and implementation of adaptation options (robust evidence, high agreement). $\{3.3\}$

Adaptation planning and implementation can be enhanced through complementary actions across levels, from individuals to governments (high confidence). National governments can coordinate adaptation efforts of local and sub-national governments, for example by protecting vulnerable groups, by supporting economic diversification and by providing information, policy and legal frameworks and financial support (robust evidence, high agreement). Local government and the private sector are increasingly recognized as critical to progress in adaptation, given their roles in scaling up adaptation of communities, households and civil society and in managing risk information and financing (medium evidence, high agreement). $\{3.3\}$

Adaptation planning and implementation at all levels of governance are contingent on societal values, objectives and risk perceptions (high confidence). Recognition of diverse interests, circumstances, social-cultural contexts and expectations can benefit decision-making processes. Indigenous, local and traditional knowledge systems and practices, including indigenous peoples' holistic view of community and environment, are a major resource for adapting to climate change, but these have not been used consistently in existing adaptation efforts. Integrating such forms of knowledge with existing practices increases the effectiveness of adaptation. $\{3.3\}$

Constraints can interact to impede adaptation planning and implementation (high confidence). Common constraints on implementation arise from the following: limited financial and human resources; limited integration or coordination of governance; uncertainties about projected impacts; different perceptions of risks; competing values; absence of key adaptation leaders and advocates; and limited tools to monitor adaptation effectiveness. Another constraint includes insufficient research, monitoring, and observation and the finance to maintain them. $\{3.3\}$ 
Greater rates and magnitude of climate change increase the likelihood of exceeding adaptation limits (high confidence). Limits to adaptation emerge from the interaction among climate change and biophysical and/or socio-economic constraints. Further, poor planning or implementation, overemphasizing short-term outcomes or failing to sufficiently anticipate consequences can result in maladaptation, increasing the vulnerability or exposure of the target group in the future or the vulnerability of other people, places or sectors (medium evidence, high agreement). Underestimating the complexity of adaptation as a social process can create unrealistic expectations about achieving intended adaptation outcomes. $\{3.3\}$

Significant co-benefits, synergies and trade-offs exist between mitigation and adaptation and among different adaptation responses; interactions occur both within and across regions (very high confidence). Increasing efforts to mitigate and adapt to climate change imply an increasing complexity of interactions, particularly at the intersections among water, energy, land use and biodiversity, but tools to understand and manage these interactions remain limited. Examples of actions with co-benefits include (i) improved energy efficiency and cleaner energy sources, leading to reduced emissions of health-damaging, climate-altering air pollutants; (ii) reduced energy and water consumption in urban areas through greening cities and recycling water; (iii) sustainable agriculture and forestry; and (iv) protection of ecosystems for carbon storage and other ecosystem services. \{3.3\}

Transformations in economic, social, technological and political decisions and actions can enhance adaptation and promote sustainable development (high confidence). At the national level, transformation is considered most effective when it reflects a country's own visions and approaches to achieving sustainable development in accordance with its national circumstances and priorities. Restricting adaptation responses to incremental changes to existing systems and structures, without considering transformational change, may increase costs and losses and miss opportunities. Planning and implementation of transformational adaptation could reflect strengthened, altered or aligned paradigms and may place new and increased demands on governance structures to reconcile different goals and visions for the future and to address possible equity and ethical implications. Adaptation pathways are enhanced by iterative learning, deliberative processes and innovation. \{3.3\}

\section{SPM 3.4 Characteristics of mitigation pathways}

There are multiple mitigation pathways that are likely to limit warming to below $2^{\circ} \mathrm{C}$ relative to pre-industrial levels. These pathways would require substantial emissions reductions over the next few decades and near zero emissions of $\mathrm{CO}_{2}$ and other long-lived greenhouse gases by the end of the century. Implementing such reductions poses substantial technological, economic, social and institutional challenges, which increase with delays in additional mitigation and if key technologies are not available. Limiting warming to lower or higher levels involves similar challenges but on different timescales. $\{3.4\}$

Without additional efforts to reduce GHG emissions beyond those in place today, global emissions growth is expected to persist, driven by growth in global population and economic activities. Global mean surface temperature increases in 2100 in baseline scenarios - those without additional mitigation-range from $3.7^{\circ} \mathrm{C}$ to $4.8^{\circ} \mathrm{C}$ above the average for $1850-1900$ for a median climate response. They range from $2.5^{\circ} \mathrm{C}$ to $7.8^{\circ} \mathrm{C}$ when including climate uncertainty (5th to 95 th percentile range) (high confidence). \{3.4\}

Emissions scenarios leading to $\mathrm{CO}_{2}$-equivalent concentrations in 2100 of about $450 \mathrm{ppm}$ or lower are likely to maintain warming below $2^{\circ} \mathrm{C}$ over the 21 st century relative to pre-industrial levels ${ }^{15}$. These scenarios are characterized by 40 to $70 \%$ global anthropogenic GHG emissions reductions by 2050 compared to $2010^{16}$, and emissions levels near zero or below in 2100. Mitigation scenarios reaching concentration levels of about $500 \mathrm{ppm} \mathrm{CO}_{2}$-eq by 2100 are more likely than not to limit temperature change to less than $2^{\circ} \mathrm{C}$, unless they temporarily overshoot concentration levels of roughly $530 \mathrm{ppm} \mathrm{CO}_{2}$-eq

For comparison, the $\mathrm{CO}_{2}$-eq concentration in 2011 is estimated to be $430 \mathrm{ppm}$ (uncertainty range 340 to 520 ppm)

16 This range differs from the range provided for a similar concentration category in the AR4 ( 50 to $85 \%$ lower than 2000 for $\mathrm{CO}_{2}$ only). Reasons for this difference include that this report has assessed a substantially larger number of scenarios than in the AR4 and looks at all GHGs. In addition, a large proportion of the new scenarios include Carbon Dioxide Removal (CDR) technologies (see below). Other factors include the use of 2100 concentration levels instead of stabilization levels and the shift in reference year from 2000 to 2010. 
before 2100, in which case they are about as likely as not to achieve that goal. In these 500 ppm $\mathrm{CO}_{2}$-eq scenarios, global 2050 emissions levels are 25 to 55\% lower than in 2010. Scenarios with higher emissions in 2050 are characterized by a greater reliance on Carbon Dioxide Removal (CDR) technologies beyond mid-century (and vice versa). Trajectories that are likely to limit warming to $3^{\circ} \mathrm{C}$ relative to pre-industrial levels reduce emissions less rapidly than those limiting warming to $2^{\circ} \mathrm{C}$. A limited number of studies provide scenarios that are more likely than not to limit warming to $1.5^{\circ} \mathrm{C}$ by 2100 ; these scenarios are characterized by concentrations below 430 ppm CO -eq by 2100 and 2050 emission reduction between $70 \%$ and $95 \%$ below 2010. For a comprehensive overview of the characteristics of emissions scenarios, their $\mathrm{CO}_{2}$-equivalent concentrations and their likelihood to keep warming to below a range of temperature levels, see Figure SPM.11 and Table SPM.1. \{3.4\}

(a) GHG emission pathways 2000-2100: All AR5 scenarios

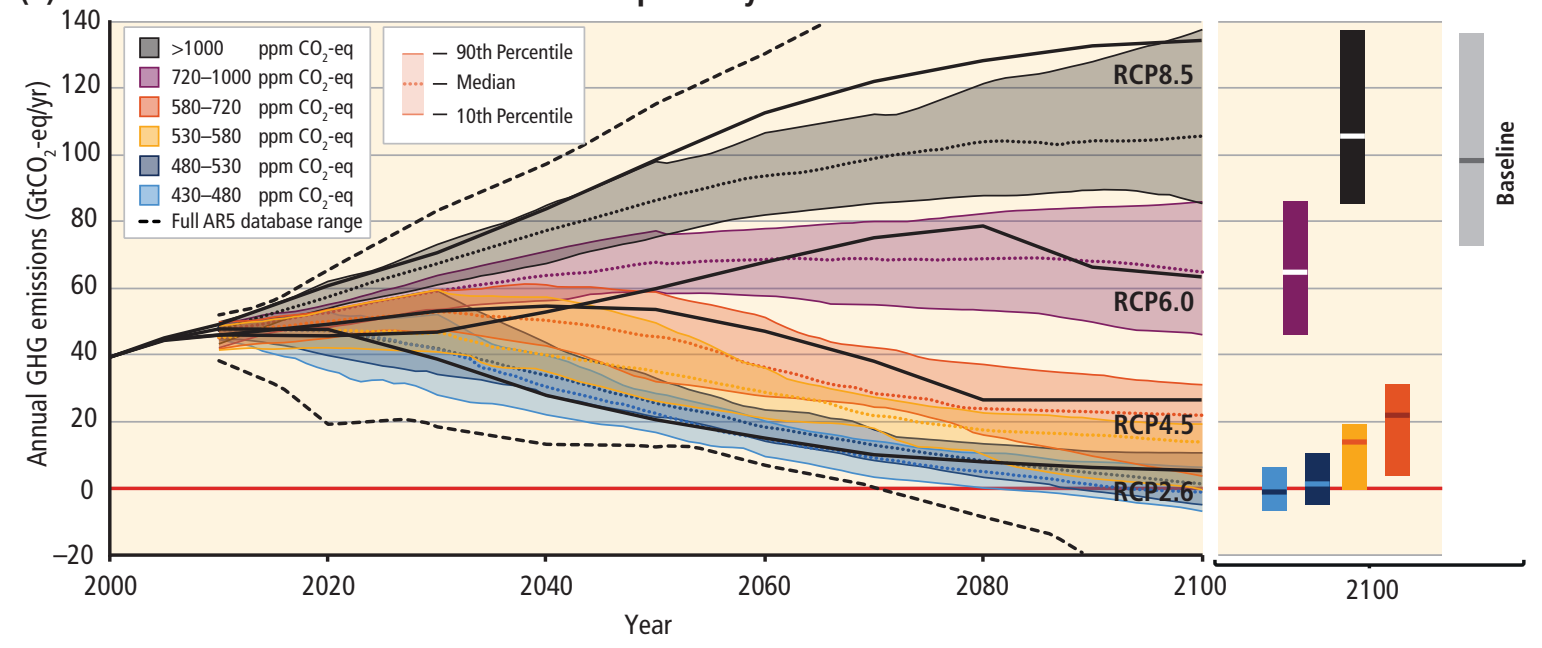

(b) Associated upscaling of low-carbon energy supply

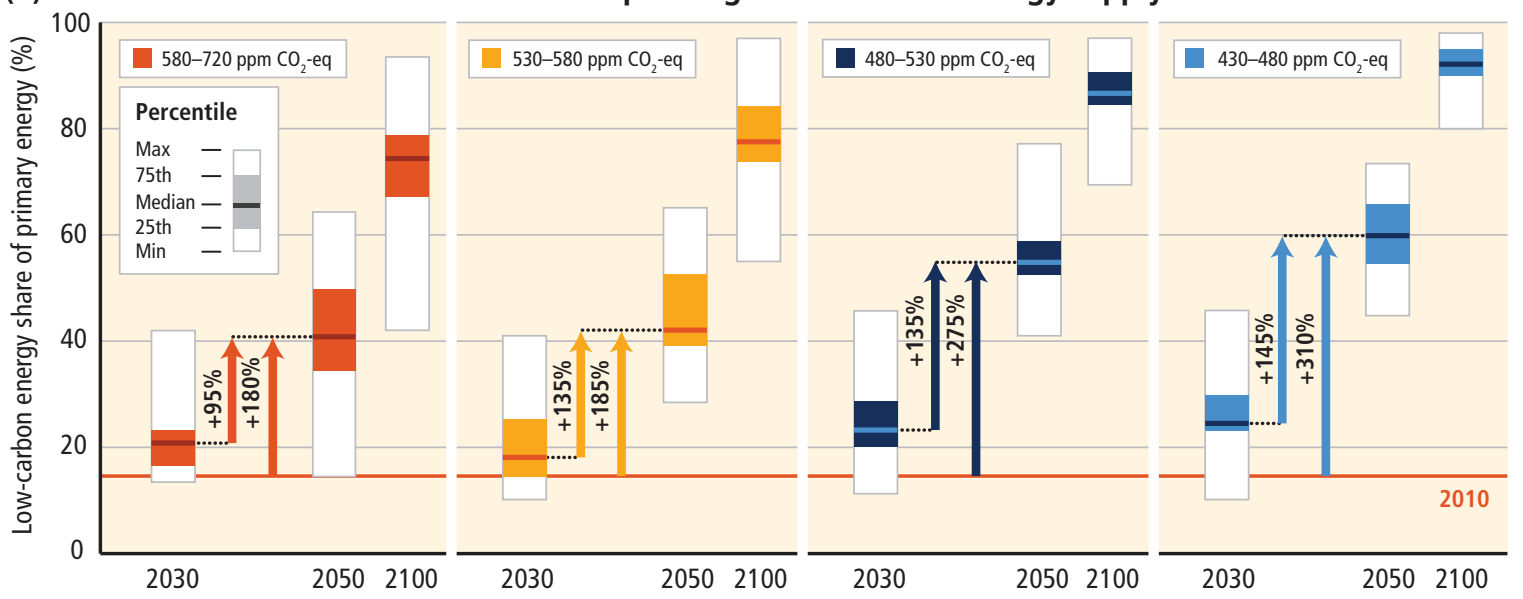

Figure SPM.11 | Global greenhouse gas emissions (gigatonne of $\mathrm{CO}_{2}$-equivalent per year, $\mathrm{GtCO}_{2}$-eq/yr) in baseline and mitigation scenarios for different long-term concentration levels (a) and associated upscaling requirements of low-carbon energy (\% of primary energy) for 2030, 2050 and 2100 compared to 2010 levels in mitigation scenarios (b). \{Figure 3.2\} 
Table SPM.1 | Key characteristics of the scenarios collected and assessed for WGIII AR5. For all parameters the 10th to 90th percentile of the scenarios is shown ${ }^{\text {a }}$ \{Table 3.1\}

\begin{tabular}{|c|c|c|c|c|c|c|c|c|}
\hline \multirow{2}{*}{$\begin{array}{c}\mathrm{CO}_{2} \text {-eq Con- } \\
\text { centrations in } \\
2100 \\
\left(p p m \mathrm{CO}_{2} \text {-eq) }\right. \\
\\
\text { Category label } \\
\text { (conc. range) }\end{array}$} & \multirow{2}{*}{ Subcategories } & \multirow{2}{*}{$\begin{array}{l}\text { Relative } \\
\text { position } \\
\text { of the } \\
\text { RCPs }{ }^{d}\end{array}$} & \multicolumn{2}{|c|}{$\begin{array}{l}\text { Change in } \mathrm{CO}_{2} \text {-eq } \\
\text { emissions compared } \\
\text { to } 2010 \text { (in \%) }\end{array}$} & \multicolumn{4}{|c|}{$\begin{array}{l}\text { Likelihood of staying below a specific } \\
\text { temperature level over the } 21 \text { st cen- } \\
\text { tury (relative to } 1850-1900)^{d, e}\end{array}$} \\
\hline & & & 2050 & 2100 & $1.5^{\circ} \mathrm{C}$ & $2^{\circ} \mathrm{C}$ & $3^{\circ} \mathrm{C}$ & $4^{\circ} \mathrm{C}$ \\
\hline$<430$ & \multicolumn{8}{|c|}{ Only a limited number of individual model studies have explored levels below $430 \mathrm{ppm} \mathrm{CO}_{2}$-eq ${ }^{\mathrm{j}}$} \\
\hline $\begin{array}{c}450 \\
\text { (430 to } 480)\end{array}$ & Total range ${ }^{a, g}$ & RCP2.6 & -72 to -41 & -118 to -78 & $\begin{array}{c}\text { More unlikely } \\
\text { than likely }\end{array}$ & Likely & \multirow{6}{*}{ Likely } & \multirow{8}{*}{ Likely } \\
\hline \multirow{2}{*}{$\begin{array}{c}500 \\
\text { (480 to } 530)\end{array}$} & $\begin{array}{l}\text { No overshoot of } \\
530 \mathrm{ppm} \mathrm{CO} \text {-eq }\end{array}$ & & -57 to -42 & -107 to -73 & \multirow{6}{*}{ Unlikely } & $\begin{array}{c}\text { More likely } \\
\text { than not }\end{array}$ & & \\
\hline & $\begin{array}{c}\text { Overshoot of } 530 \\
\text { ppm } \mathrm{CO}_{2} \text {-eq }\end{array}$ & & -55 to -25 & -114 to -90 & & $\begin{array}{l}\text { About as } \\
\text { likely as not }\end{array}$ & & \\
\hline \multirow{2}{*}{$\begin{array}{c}550 \\
\text { (530 to } 580 \text { ) }\end{array}$} & $\begin{array}{l}\text { No overshoot of } \\
580 \mathrm{ppm} \mathrm{CO} \mathrm{CO}_{2} \text {-eq }\end{array}$ & & -47 to -19 & -81 to -59 & & \multirow{3}{*}{$\begin{array}{c}\text { More unlikely } \\
\text { than likely }{ }^{i}\end{array}$} & & \\
\hline & $\begin{array}{l}\text { Overshoot of } 580 \\
\text { ppm } \mathrm{CO}_{2} \text {-eq }\end{array}$ & & -16 to 7 & -183 to -86 & & & & \\
\hline (580 to 650$)$ & Total range & \multirow{2}{*}{ RCP4.5 } & -38 to 24 & -134 to -50 & & & & \\
\hline (650 to 720 ) & Total range & & -11 to 17 & -54 to -21 & & \multirow[t]{2}{*}{ Unlikely } & $\begin{array}{c}\text { More likely } \\
\text { than not }\end{array}$ & \\
\hline (720 to 1000$)^{b}$ & Total range & RCP6.0 & 18 to 54 & -7 to 72 & \multirow{2}{*}{ Unlikely ${ }^{h}$} & & $\begin{array}{c}\text { More unlikely } \\
\text { than likely }\end{array}$ & \\
\hline$>1000^{b}$ & Total range & RCP8.5 & 52 to 95 & 74 to 178 & & Unlikely ${ }^{h}$ & Unlikely & $\begin{array}{c}\text { More unlikely } \\
\text { than likely }\end{array}$ \\
\hline
\end{tabular}

Notes:

a The 'total range' for the 430 to $480 \mathrm{ppm} \mathrm{CO}$-eq concentrations scenarios corresponds to the range of the 10th to 90th percentile of the subcategory of these scenarios shown in Table 6.3 of the Working Group III Report.

${ }^{\mathrm{b}}$ Baseline scenarios fall into the $>1000$ and 720 to $1000 \mathrm{ppm} \mathrm{CO}$-eq categories. The latter category also includes mitigation scenarios. The baseline scenarios in the latter category reach a temperature change of $2.5^{\circ} \mathrm{C}$ to $5.8^{\circ} \mathrm{C}$ above the average for $1850-1900$ in 2100 . Together with the baseline scenarios in the $>1000 \mathrm{ppm} \mathrm{CO}_{2}$-eq category, this leads to an overall 2100 temperature range of $2.5^{\circ} \mathrm{C}$ to $7.8^{\circ} \mathrm{C}$ (range based on median climate response: $3.7^{\circ} \mathrm{C}$ to $4.8^{\circ} \mathrm{C}$ ) for baseline scenarios across both concentration categories.

c The global 2010 emissions are 31\% above the 1990 emissions (consistent with the historic greenhouse gas emission estimates presented in this report). $\mathrm{CO}_{2}$-eq emissions include the basket of Kyoto gases (carbon dioxide $\left(\mathrm{CO}_{2}\right)$, methane $\left(\mathrm{CH}_{4}\right)$, nitrous oxide $\left(\mathrm{N}_{2} \mathrm{O}\right)$ as well as fluorinated gases).

${ }^{d}$ The assessment here involves a large number of scenarios published in the scientific literature and is thus not limited to the Representative Concentration Pathways (RCPs). To evaluate the $\mathrm{CO}_{2}$-eq concentration and climate implications of these scenarios, the Model for the Assessment of Greenhouse Gas Induced Climate Change (MAGICC) was used in a probabilistic mode. For a comparison between MAGICC model results and the outcomes of the models used in WGI, see WGI 12.4.1.2, 12.4.8 and WGIII 6.3.2.6.

${ }^{\mathrm{e}}$ The assessment in this table is based on the probabilities calculated for the full ensemble of scenarios in WGIII AR5 using MAGICC and the assessment in WGI of the uncertainty of the temperature projections not covered by climate models. The statements are therefore consistent with the statements in WGI, which are based on the Coupled Model Intercomparison Project Phase 5 (CMIP5) runs of the RCPs and the assessed uncertainties. Hence, the likelihood statements reflect different lines of evidence from both WGs. This WGI method was also applied for scenarios with intermediate concentration levels where no CMIP5 runs are available. The likelihood statements are indicative only \{WGIII 6.3\} and follow broadly the terms used by the WGI SPM for temperature projections: likely $66-100 \%$, more likely than not $>50-100 \%$, about as likely as not $33-66 \%$, and unlikely $0-33 \%$. In addition the term more unlikely than likely $0-<50 \%$ is used.

${ }^{f}$ The $\mathrm{CO}_{2}$-equivalent concentration (see Glossary) is calculated on the basis of the total forcing from a simple carbon cycle/climate model, $\mathrm{MAGICC}$. The $\mathrm{CO}_{2}$ equivalent concentration in 2011 is estimated to be $430 \mathrm{ppm}$ (uncertainty range 340 to $520 \mathrm{ppm}$ ). This is based on the assessment of total anthropogenic radiative forcing for 2011 relative to $1750 \mathrm{in} \mathrm{WGl}$, i.e., $2.3 \mathrm{~W} / \mathrm{m}^{2}$, uncertainty range 1.1 to $3.3 \mathrm{~W} / \mathrm{m}^{2}$.

9 The vast majority of scenarios in this category overshoot the category boundary of $480 \mathrm{ppm} \mathrm{CO}_{2}$-eq concentration.

h For scenarios in this category, no CMIP5 run or MAGICC realization stays below the respective temperature level. Still, an unlikely assignment is given to reflect uncertainties that may not be reflected by the current climate models.

i Scenarios in the 580 to $650 \mathrm{ppm} \mathrm{CO}$-eq category include both overshoot scenarios and scenarios that do not exceed the concentration level at the high end of the category (e.g., RCP4.5). The latter type of scenarios, in general, have an assessed probability of more unlikely than likely to stay below the $2^{\circ} \mathrm{C}$ temperature level, while the former are mostly assessed to have an unlikely probability of staying below this level.

i In these scenarios, global $\mathrm{CO}_{2}$-eq emissions in 2050 are between 70 to 95\% below 2010 emissions, and they are between 110 to $120 \%$ below 2010 emissions in 2100. 
Before 2030

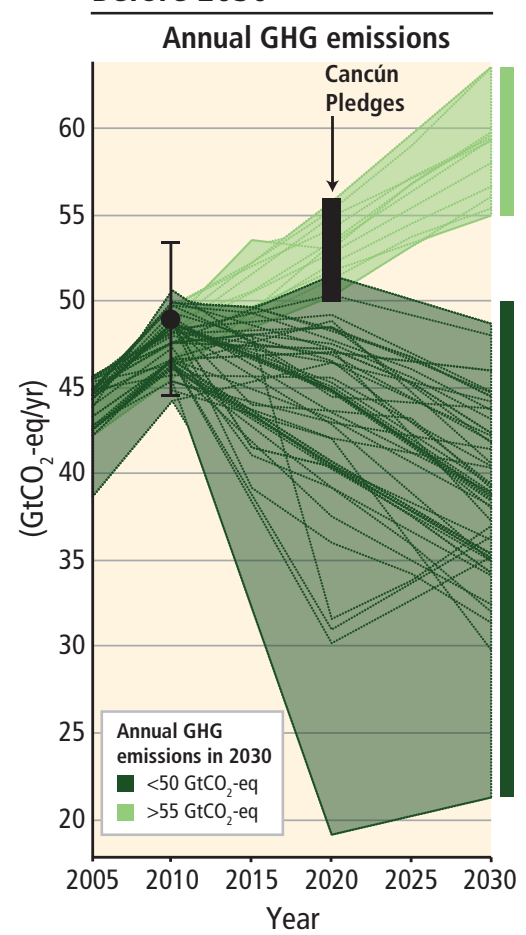

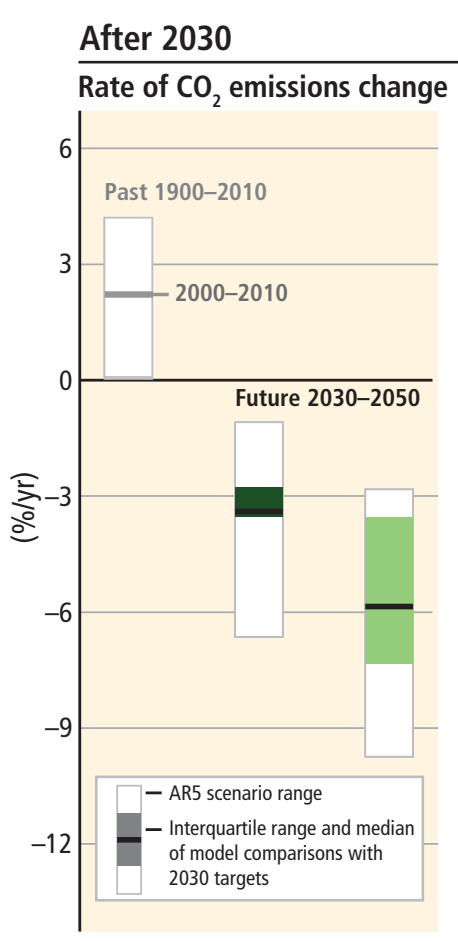

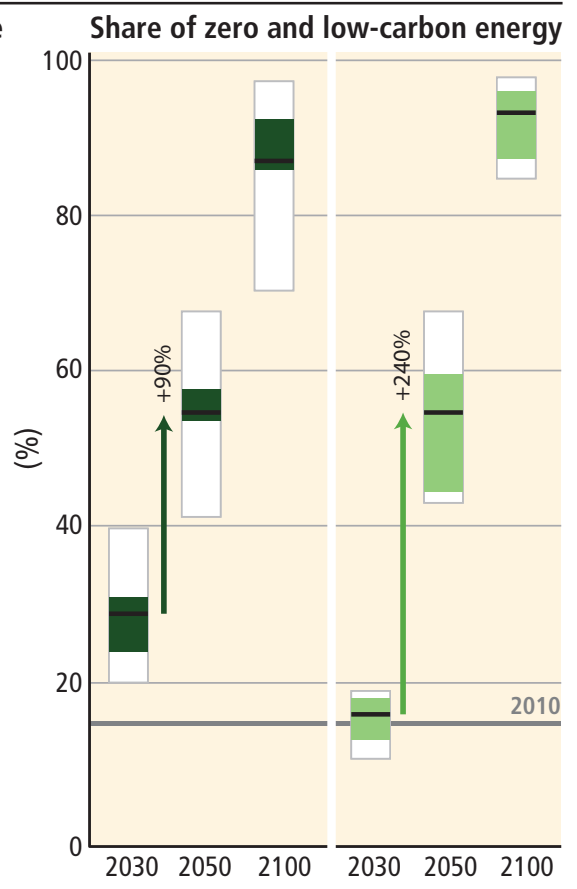

Figure SPM.12 | The implications of different 2030 greenhouse gas (GHG) emissions levels for the rate of carbon dioxide $\left(\mathrm{CO}_{2}\right)$ emissions reductions and low-carbon energy upscaling in mitigation scenarios that are at least about as likely as not to keep warming throughout the $21 \mathrm{st}$ century below $2^{\circ} \mathrm{C}$ relative to pre-industrial levels ( $2100 \mathrm{CO}_{2}$-equivalent concentrations of 430 to $530 \mathrm{ppm}$ ). The scenarios are grouped according to different emissions levels by 2030 (coloured in different shades of green). The left panel shows the pathways of $\mathrm{GHG}$ emissions (gigatonne of $\mathrm{CO}_{2}$-equivalent per year, $\mathrm{GtCO}_{2}$-eq/ $\mathrm{yr}$ ) leading to these 2030 levels. The black dot with whiskers gives historic GHG emission levels and associated uncertainties in 2010 as reported in Figure SPM.2. The black bar shows the estimated uncertainty range of GHG emissions implied by the Cancún Pledges. The middle panel denotes the average annual $\mathrm{CO}_{2}$ emissions reduction rates for the period 2030-2050. It compares the median and interquartile range across scenarios from recent inter-model comparisons with explicit 2030 interim goals to the range of scenarios in the Scenario Database for WGIII AR5. Annual rates of historical emissions change (sustained over a period of 20 years) and the average annual $\mathrm{CO}_{2}$ emission change between 2000 and 2010 are shown as well. The arrows in the right panel show the magnitude of zero and low-carbon energy supply upscaling from 2030 to 2050 subject to different $2030 \mathrm{GHG}$ emissions levels. Zero- and low-carbon energy supply includes renewables, nuclear energy and fossil energy with carbon dioxide capture and storage (CCS) or bioenergy with CCS (BECCS). [Note: Only scenarios that apply the full, unconstrained mitigation technology portfolio of the underlying models (default technology assumption) are shown. Scenarios with large net negative global emissions ( $>20 \mathrm{GtCO}_{2}$-eq/yr), scenarios with exogenous carbon price assumptions and scenarios with 2010 emissions significantly outside the historical range are excluded.] \{Figure 3.3\}

Mitigation scenarios reaching about $450 \mathrm{ppm} \mathrm{CO}$-eq in 2100 (consistent with a likely chance to keep warming below $2^{\circ} \mathrm{C}$ relative to pre-industrial levels) typically involve temporary overshoot ${ }^{17}$ of atmospheric concentrations, as do many scenarios reaching about $500 \mathrm{ppm} \mathrm{CO}$-eq to about $550 \mathrm{ppm} \mathrm{CO}$-eq in 2100 (Table SPM.1). Depending on the level of overshoot, overshoot scenarios typically rely on the availability and widespread deployment of bioenergy with carbon dioxide capture and storage (BECCS) and afforestation in the second half of the century. The availability and scale of these and other CDR technologies and methods are uncertain and CDR technologies are, to varying degrees, associated with challenges and risks ${ }^{18}$. CDR is also prevalent in many scenarios without overshoot to compensate for residual emissions from sectors where mitigation is more expensive (high confidence). $\{3.4$, Box 3.3$\}$

Reducing emissions of non- $\mathrm{CO}_{2}$ agents can be an important element of mitigation strategies. All current GHG emissions and other forcing agents affect the rate and magnitude of climate change over the next few decades, although long-term warming is mainly driven by $\mathrm{CO}_{2}$ emissions. Emissions of non- $\mathrm{CO}_{2}$ forcers are often expressed as ' $\mathrm{CO}_{2}$-equivalent emissions', but the choice of metric to calculate these emissions, and the implications for the emphasis and timing of abatement of the various climate forcers, depends on application and policy context and contains value judgments. $\{3.4$, Box 3.2$\}$

17 In concentration 'overshoot' scenarios, concentrations peak during the century and then decline.

18 CDR methods have biogeochemical and technological limitations to their potential on the global scale. There is insufficient knowledge to quantify how much $\mathrm{CO}_{2}$ emissions could be partially offset by CDR on a century timescale. CDR methods may carry side effects and long-term consequences on a global scale. 


\section{Global mitigation costs and consumption growth in baseline scenarios}
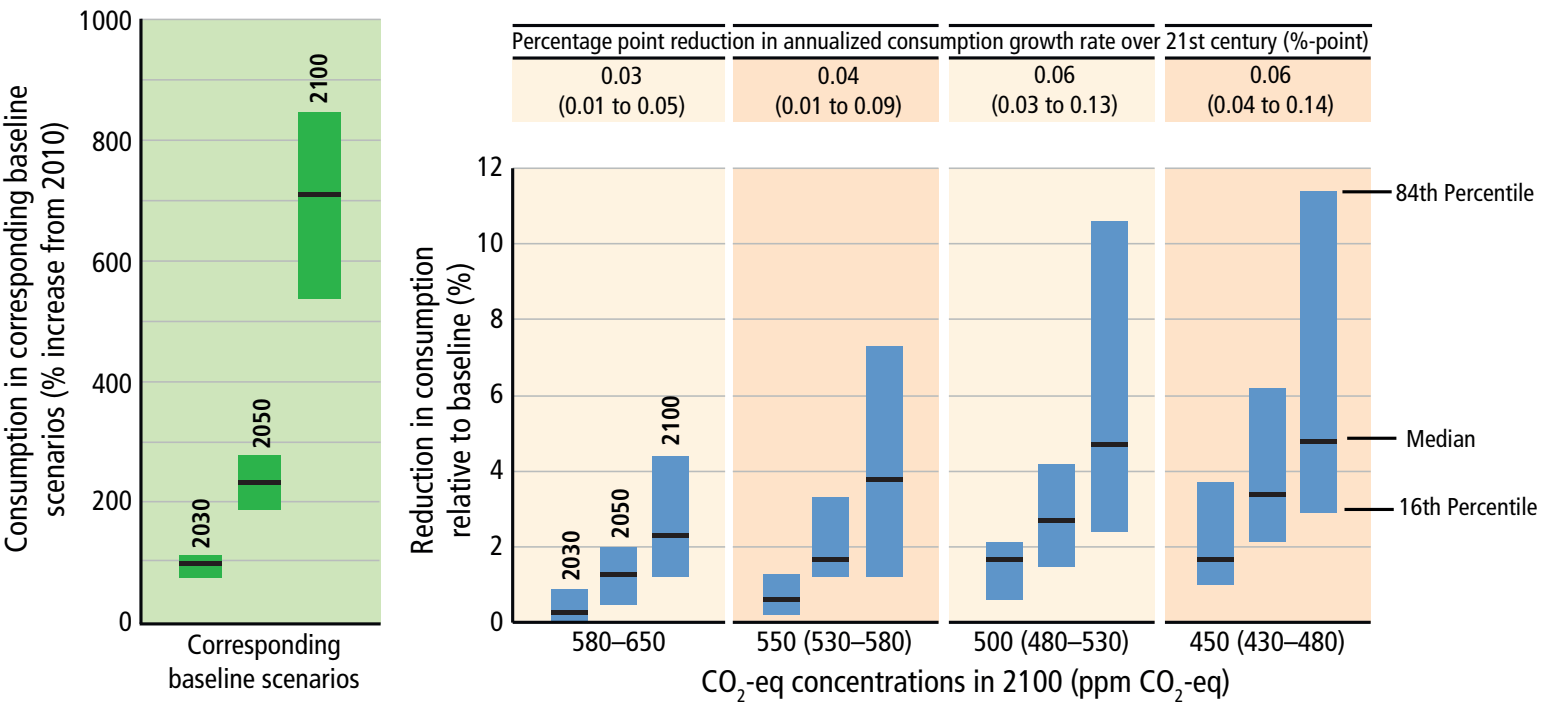

Figure SPM.13 | Global mitigation costs in cost-effective scenarios at different atmospheric concentrations levels in 2100. Cost-effective scenarios assume immediate mitigation in all countries and a single global carbon price, and impose no additional limitations on technology relative to the models' default technology assumptions. Consumption losses are shown relative to a baseline development without climate policy (left panel). The table at the top shows percentage points of annualized consumption growth reductions relative to consumption growth in the baseline of 1.6 to $3 \%$ per year (e.g., if the reduction is 0.06 percentage points per year due to mitigation, and baseline growth is $2.0 \%$ per year, then the growth rate with mitigation would be $1.94 \%$ per year). Cost estimates shown in this table do not consider the benefits of reduced climate change or co-benefits and adverse side effects of mitigation. Estimates at the high end of these cost ranges are from models that are relatively inflexible to achieve the deep emissions reductions required in the long run to meet these goals and/or include assumptions about market imperfections that would raise costs. \{Figure 3.4\}

Delaying additional mitigation to 2030 will substantially increase the challenges associated with limiting warming over the 21 st century to below $2^{\circ} \mathrm{C}$ relative to pre-industrial levels. It will require substantially higher rates of emissions reductions from 2030 to 2050; a much more rapid scale-up of low-carbon energy over this period; a larger reliance on CDR in the long term; and higher transitional and long-term economic impacts. Estimated global emissions levels in 2020 based on the Cancún Pledges are not consistent with cost-effective mitigation trajectories that are at least about as likely as not to limit warming to below $2^{\circ} \mathrm{C}$ relative to pre-industrial levels, but they do not preclude the option to meet this goal (high confidence) (Figure SPM.12, Table SPM.2). \{3.4\}

Estimates of the aggregate economic costs of mitigation vary widely depending on methodologies and assumptions, but increase with the stringency of mitigation. Scenarios in which all countries of the world begin mitigation immediately, in which there is a single global carbon price, and in which all key technologies are available have been used as a cost-effective benchmark for estimating macro-economic mitigation costs (Figure SPM.13). Under these assumptions mitigation scenarios that are likely to limit warming to below $2^{\circ} \mathrm{C}$ through the 21 st century relative to pre-industrial levels entail losses in global consumption - not including benefits of reduced climate change as well as co-benefits and adverse side effects of mitigation—of 1 to $4 \%$ (median: 1.7\%) in 2030, 2 to $6 \%$ (median: 3.4\%) in 2050 and 3 to $11 \%$ (median: $4.8 \%$ ) in 2100 relative to consumption in baseline scenarios that grows anywhere from $300 \%$ to more than $900 \%$ over the century (Figure SPM.13). These numbers correspond to an annualized reduction of consumption growth by 0.04 to 0.14 (median: 0.06 ) percentage points over the century relative to annualized consumption growth in the baseline that is between 1.6 and $3 \%$ per year (high confidence). $\{3.4\}$

In the absence or under limited availability of mitigation technologies (such as bioenergy, CCS and their combination BECCS, nuclear, wind/solar), mitigation costs can increase substantially depending on the technology considered. Delaying additional mitigation increases mitigation costs in the medium to long term. Many models could not limit likely warming to below $2^{\circ} \mathrm{C}$ over the 21st century relative to pre-industrial levels if additional mitigation is considerably delayed. Many models could not limit likely warming to below $2^{\circ} \mathrm{C}$ if bioenergy, CCS and their combination (BECCS) are limited (high confidence) (Table SPM.2). \{3.4\} 
Table SPM.2 | Increase in global mitigation costs due to either limited availability of specific technologies or delays in additional mitigation a relative to cost-effective scenarios $^{b}$. The increase in costs is given for the median estimate and the 16 th to 84 th percentile range of the scenarios (in parentheses) ${ }^{c}$. In addition, the sample size of each scenario set is provided in the coloured symbols. The colours of the symbols indicate the fraction of models from systematic model comparison exercises that could successfully reach the targeted concentration level. \{Table 3.2\}

\begin{tabular}{|c|c|c|c|c|c|c|}
\hline & $\begin{array}{r}\text { Mitigation } \\
\text { limited } \\
\text { [\% increase } \\
(2015-2100) \text { rel }\end{array}$ & $\begin{array}{l}\text { cost increases in } \\
\text { availability of tec } \\
\text { total discounted } \\
\text { tive to default tech }\end{array}$ & $\begin{array}{l}\text { cenarios with } \\
\text { mologies }{ }^{d} \\
\text { mitigation costs } \\
\text { nology assumption }\end{array}$ & & $\begin{array}{r}\text { Mitigation co } \\
\text { due to delaye } \\
\text { mitigation } \\
\text { [\% increase in m } \\
\text { relative to immed }\end{array}$ & $\begin{array}{l}\text { t increases } \\
\text { l additional } \\
\text { ntil } 2030 \\
\text { tigation costs } \\
\text { ate mitigation] }\end{array}$ \\
\hline $\begin{array}{c}2100 \\
\text { concentrations } \\
\text { (ppm CO} \mathrm{CO}_{2} \text {-eq) }\end{array}$ & no CCS & nuclear phase out & limited solar/wind & limited bioenergy & $\begin{array}{l}\text { medium term costs } \\
\qquad(2030-2050)\end{array}$ & $\begin{array}{l}\text { long term } \\
\text { costs } \\
(2050-2100)\end{array}$ \\
\hline $\begin{array}{c}450 \\
\text { (430 to } 480)\end{array}$ & $\begin{array}{c}138 \% \\
(29 \text { to } 297 \%)\end{array}$ & $\begin{array}{c}7 \% \\
(4 \text { to } 18 \%)\end{array}$ & $\begin{array}{c}6 \% \\
(2 \text { to } 29 \%) \quad 8\end{array}$ & $\begin{array}{c}64 \% \\
(44 \text { to } 78 \%)\end{array}$ & $44 \%$ & $37 \%$ \\
\hline $\begin{array}{c}500 \\
\text { (480 to } 530)\end{array}$ & $\begin{array}{l}\text { not available } \\
\text { (n.a.) }\end{array}$ & n.a. & n.a. & n.a. & & \\
\hline $\begin{array}{c}550 \\
\text { (530 to 580) }\end{array}$ & $\begin{array}{c}39 \% \\
(18 \text { to } 78 \%) \quad 11\end{array}$ & $\begin{array}{c}13 \% \\
(2 \text { to } 23 \%)\end{array}$ & $\begin{array}{c}8 \% \\
\text { (5 to } 15 \%)\end{array}$ & $\begin{array}{c}18 \% \\
\text { (4 to } 66 \%) \quad 12\end{array}$ & $15 \%$ & $16 \%$ \\
\hline 580 to 650 & n.a. & n.a. & n.a. & n.a. & & \\
\hline Symbol legend- & fraction of models su & ccessful in producin & scenarios (numbe & rs indicate the num & successful models) & \\
\hline $\begin{array}{l}\mathbb{E} \text { : all models st } \\
=\text { : between } 80\end{array}$ & cessful $100 \%$ of models suc & essful & & 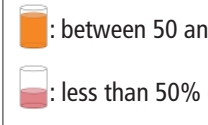 & $\begin{array}{l}\text { of models successful } \\
\text { dels successful }\end{array}$ & \\
\hline
\end{tabular}

Notes:

a Delayed mitigation scenarios are associated with greenhouse gas emission of more than $55 \mathrm{GtCO}_{2}$-eq in 2030, and the increase in mitigation costs is measured relative to cost-effective mitigation scenarios for the same long-term concentration level.

${ }^{b}$ Cost-effective scenarios assume immediate mitigation in all countries and a single global carbon price, and impose no additional limitations on technology relative to the models' default technology assumptions.

c The range is determined by the central scenarios encompassing the 16th to 84th percentile range of the scenario set. Only scenarios with a time horizon until 2100 are included. Some models that are included in the cost ranges for concentration levels above 530 ppm CO $\mathrm{CO}_{2}$-eq in 2100 could not produce associated scenarios for concentration levels below 530 ppm CO $\mathrm{C}_{2}$-eq in 2100 with assumptions about limited availability of technologies and/or delayed additional mitigation.

${ }^{\mathrm{d}}$ No CCS: carbon dioxide capture and storage is not included in these scenarios. Nuclear phase out: no addition of nuclear power plants beyond those under construction, and operation of existing plants until the end of their lifetime. Limited Solar/Wind: a maximum of $20 \%$ global electricity generation from solar and wind power in any year of these scenarios. Limited Bioenergy: a maximum of $100 \mathrm{EJ} / \mathrm{yr}$ modern bioenergy supply globally (modern bioenergy used for heat, power, combinations and industry was around $18 \mathrm{EJ} / \mathrm{yr}$ in 2008). EJ $=$ Exajoule $=10^{18} \mathrm{Joule}$.

e Percentage increase of net present value of consumption losses in percent of baseline consumption (for scenarios from general equilibrium models) and abatement costs in percent of baseline gross domestic product (GDP, for scenarios from partial equilibrium models) for the period 2015-2100, discounted at $5 \%$ per year.

Mitigation scenarios reaching about 450 or 500 ppm $\mathrm{CO}_{2}$-eq by 2100 show reduced costs for achieving air quality and energy security objectives, with significant co-benefits for human health, ecosystem impacts and sufficiency of resources and resilience of the energy system. $\{4.4 .2 .2\}$

Mitigation policy could devalue fossil fuel assets and reduce revenues for fossil fuel exporters, but differences between regions and fuels exist (high confidence). Most mitigation scenarios are associated with reduced revenues from coal and oil trade for major exporters (high confidence). The availability of CCS would reduce the adverse effects of mitigation on the value of fossil fuel assets (medium confidence). \{4.4.2.2\}

Solar Radiation Management (SRM) involves large-scale methods that seek to reduce the amount of absorbed solar energy in the climate system. SRM is untested and is not included in any of the mitigation scenarios. If it were deployed, SRM would 
entail numerous uncertainties, side effects, risks and shortcomings and has particular governance and ethical implications. SRM would not reduce ocean acidification. If it were terminated, there is high confidence that surface temperatures would rise very rapidly impacting ecosystems susceptible to rapid rates of change. \{Box 3.3$\}$

\section{SPM 4. Adaptation and Mitigation}

Many adaptation and mitigation options can help address climate change, but no single option is sufficient by itself. Effective implementation depends on policies and cooperation at all scales and can be enhanced through integrated responses that link adaptation and mitigation with other societal objectives. $\{4\}$

\section{SPM 4.1 Common enabling factors and constraints for adaptation and mitigation responses}

Adaptation and mitigation responses are underpinned by common enabling factors. These include effective institutions and governance, innovation and investments in environmentally sound technologies and infrastructure, sustainable livelihoods and behavioural and lifestyle choices. $\{4.1\}$

Inertia in many aspects of the socio-economic system constrains adaptation and mitigation options (medium evidence, high agreement). Innovation and investments in environmentally sound infrastructure and technologies can reduce GHG emissions and enhance resilience to climate change (very high confidence). $\{4.1\}$

Vulnerability to climate change, GHG emissions and the capacity for adaptation and mitigation are strongly influenced by livelihoods, lifestyles, behaviour and culture (medium evidence, medium agreement). Also, the social acceptability and/or effectiveness of climate policies are influenced by the extent to which they incentivize or depend on regionally appropriate changes in lifestyles or behaviours. $\{4.1\}$

For many regions and sectors, enhanced capacities to mitigate and adapt are part of the foundation essential for managing climate change risks (high confidence). Improving institutions as well as coordination and cooperation in governance can help overcome regional constraints associated with mitigation, adaptation and disaster risk reduction (very high confidence). $\{4.1\}$

\section{SPM 4.2 Response options for adaptation}

Adaptation options exist in all sectors, but their context for implementation and potential to reduce climate-related risks differs across sectors and regions. Some adaptation responses involve significant co-benefits, synergies and trade-offs. Increasing climate change will increase challenges for many adaptation options. $\{4.2\}$

Adaptation experience is accumulating across regions in the public and private sectors and within communities. There is increasing recognition of the value of social (including local and indigenous), institutional, and ecosystem-based measures and of the extent of constraints to adaptation. Adaptation is becoming embedded in some planning processes, with more limited implementation of responses (high confidence). $\{1.6,4.2,4.4 .2 .1\}$

The need for adaptation along with associated challenges is expected to increase with climate change (very high confidence). Adaptation options exist in all sectors and regions, with diverse potential and approaches depending on their context in vulnerability reduction, disaster risk management or proactive adaptation planning (Table SPM.3). Effective strategies and actions consider the potential for co-benefits and opportunities within wider strategic goals and development plans. $\{4.2\}$ 
Table SPM.3 | Approaches for managing the risks of climate change through adaptation. These approaches should be considered overlapping rather than discrete, and they are often pursued simultaneously. Examples are presented in no specific order and can be relevant to more than one category. \{Table 4.2\}

\begin{tabular}{|c|c|c|c|c|}
\hline & $\begin{array}{l}\text { Ierlappir } \\
\text { pproach }\end{array}$ & & Category & Examples \\
\hline 들 쓸 & & & $\begin{array}{l}\text { Human } \\
\text { development }\end{array}$ & $\begin{array}{l}\text { Improved access to education, nutrition, health facilities, energy, safe housing \& settlement structures, } \\
\text { \& social support structures; Reduced gender inequality \& marginalization in other forms. }\end{array}$ \\
\hline 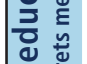 & & & Poverty alleviation & $\begin{array}{l}\text { Improved access to \& control of local resources; Land tenure; Disaster risk reduction; Social safety nets } \\
\& \text { social protection; Insurance schemes. }\end{array}$ \\
\hline 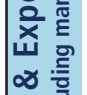 & & & $\begin{array}{l}\text { Disaster risk } \\
\text { management }\end{array}$ & $\begin{array}{l}\text { Early warning systems; Hazard \& vulnerability mapping; Diversifying water resources; Improved } \\
\text { drainage; Flood \& cyclone shelters; Building codes \& practices; Storm \& wastewater management; } \\
\text { Transport \& road infrastructure improvements. }\end{array}$ \\
\hline 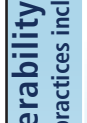 & & & $\begin{array}{l}\text { Ecosystem } \\
\text { management }\end{array}$ & $\begin{array}{l}\text { Maintaining wetlands \& urban green spaces; Coastal afforestation; Watershed \& reservoir } \\
\text { management; Reduction of other stressors on ecosystems \& of habitat fragmentation; Maintenance } \\
\text { of genetic diversity; Manipulation of disturbance regimes; Community-based natural resource } \\
\text { management. }\end{array}$ \\
\hline 흠 & & & & $\begin{array}{l}\text { Engineered \& built-environment options: Sea walls \& coastal protection structures; Flood levees; } \\
\text { Water storage; Improved drainage; Flood \& cyclone shelters; Building codes \& practices; Storm \& } \\
\text { wastewater management; Transport \& road infrastructure improvements; Floating houses; Power plant } \\
\text { \& electricity grid adjustments. }\end{array}$ \\
\hline 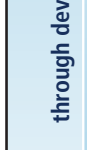 & & & Structural/physical & $\begin{array}{l}\text { Technological options: New crop \& animal varieties; Indigenous, traditional \& local knowledge, } \\
\text { technologies \& methods; Efficient irrigation; Water-saving technologies; Desalinisation; Conservation } \\
\text { agriculture; Food storage \& preservation facilities; Hazard \& vulnerability mapping \& monitoring; Early } \\
\text { warning systems; Building insulation; Mechanical \& passive cooling; Technology development, transfer } \\
\text { \& diffusion. }\end{array}$ \\
\hline & 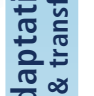 & & & $\begin{array}{l}\text { Economic options: Financial incentives; Insurance; Catastrophe bonds; Payments for ecosystem } \\
\text { services; Pricing water to encourage universal provision and careful use; Microfinance; Disaster } \\
\text { contingency funds; Cash transfers; Public-private partnerships. }\end{array}$ \\
\hline & $<\underset{\frac{\pi}{c}}{\stackrel{c}{d}}$ & & Institutional & $\begin{array}{l}\text { Laws \& regulations: Land zoning laws; Building standards \& practices; Easements; Water regulations } \\
\text { \& agreements; Laws to support disaster risk reduction; Laws to encourage insurance purchasing; } \\
\text { Defined property rights \& land tenure security; Protected areas; Fishing quotas; Patent pools \& } \\
\text { technology transfer. }\end{array}$ \\
\hline & 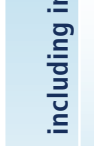 & & & $\begin{array}{l}\text { National \& government policies \& programs: National \& regional adaptation plans including } \\
\text { mainstreaming; Sub-national \& local adaptation plans; Economic diversification; Urban upgrading } \\
\text { programs; Municipal water management programs; Disaster planning \& preparedness; Integrated } \\
\text { water resource management; Integrated coastal zone management; Ecosystem-based management; } \\
\text { Community-based adaptation. }\end{array}$ \\
\hline & & & & $\begin{array}{l}\text { Educational options: Awareness raising \& integrating into education; Gender equity in education; } \\
\text { Extension services; Sharing indigenous, traditional \& local knowledge; Participatory action research \& } \\
\text { social learning; Knowledge-sharing \& learning platforms. }\end{array}$ \\
\hline & & & Social & $\begin{array}{l}\text { Informational options: Hazard \& vulnerability mapping; Early warning \& response systems; } \\
\text { Systematic monitoring \& remote sensing; Climate services; Use of indigenous climate observations; } \\
\text { Participatory scenario development; Integrated assessments. }\end{array}$ \\
\hline & & & & $\begin{array}{l}\text { Behavioural options: Household preparation \& evacuation planning; Migration; Soil \& water } \\
\text { conservation; Storm drain clearance; Livelihood diversification; Changed cropping, livestock \& } \\
\text { aquaculture practices; Reliance on social networks. }\end{array}$ \\
\hline
\end{tabular}




\section{SPM 4.3 Response options for mitigation}

Mitigation options are available in every major sector. Mitigation can be more cost-effective if using an integrated approach that combines measures to reduce energy use and the greenhouse gas intensity of end-use sectors, decarbonize energy supply, reduce net emissions and enhance carbon sinks in land-based sectors. $\{4.3\}$

Well-designed systemic and cross-sectoral mitigation strategies are more cost-effective in cutting emissions than a focus on individual technologies and sectors, with efforts in one sector affecting the need for mitigation in others (medium confidence). Mitigation measures intersect with other societal goals, creating the possibility of co-benefits or adverse side effects. These intersections, if well-managed, can strengthen the basis for undertaking climate action. $\{4.3\}$

Emissions ranges for baseline scenarios and mitigation scenarios that limit $\mathrm{CO}_{2}$-equivalent concentrations to low levels (about $450 \mathrm{ppm} \mathrm{CO} \mathrm{CO}_{2}$-eq, likely to limit warming to $2^{\circ} \mathrm{C}$ above pre-industrial levels) are shown for different sectors and gases in Figure SPM.14. Key measures to achieve such mitigation goals include decarbonizing (i.e., reducing the carbon intensity of) electricity generation (medium evidence, high agreement) as well as efficiency enhancements and behavioural changes, in order to reduce energy demand compared to baseline scenarios without compromising development (robust evidence, high agreement). In scenarios reaching $450 \mathrm{ppm} \mathrm{CO}_{2}$-eq concentrations by 2100 , global $\mathrm{CO}_{2}$ emissions from the energy supply sector are projected to decline over the next decade and are characterized by reductions of $90 \%$ or more below 2010 levels between 2040 and 2070. In the majority of low-concentration stabilization scenarios (about 450 to about $500 \mathrm{ppm} \mathrm{CO}$-eq, at least about as likely as not to limit warming to $2^{\circ} \mathrm{C}$ above pre-industrial levels), the share of low-carbon electricity supply (comprising renewable energy (RE), nuclear and carbon dioxide capture and storage (CCS) including bioenergy with carbon dioxide capture and storage (BECCS)) increases from the current share of approximately $30 \%$ to more than $80 \%$ by 2050 , and fossil fuel power generation without CCS is phased out almost entirely by $2100 .\{4.3\}$

Direct $\mathrm{CO}_{2}$ emissions by major sectors, and non- $\mathrm{CO}_{2}$ emissions, for baseline and mitigation scenarios

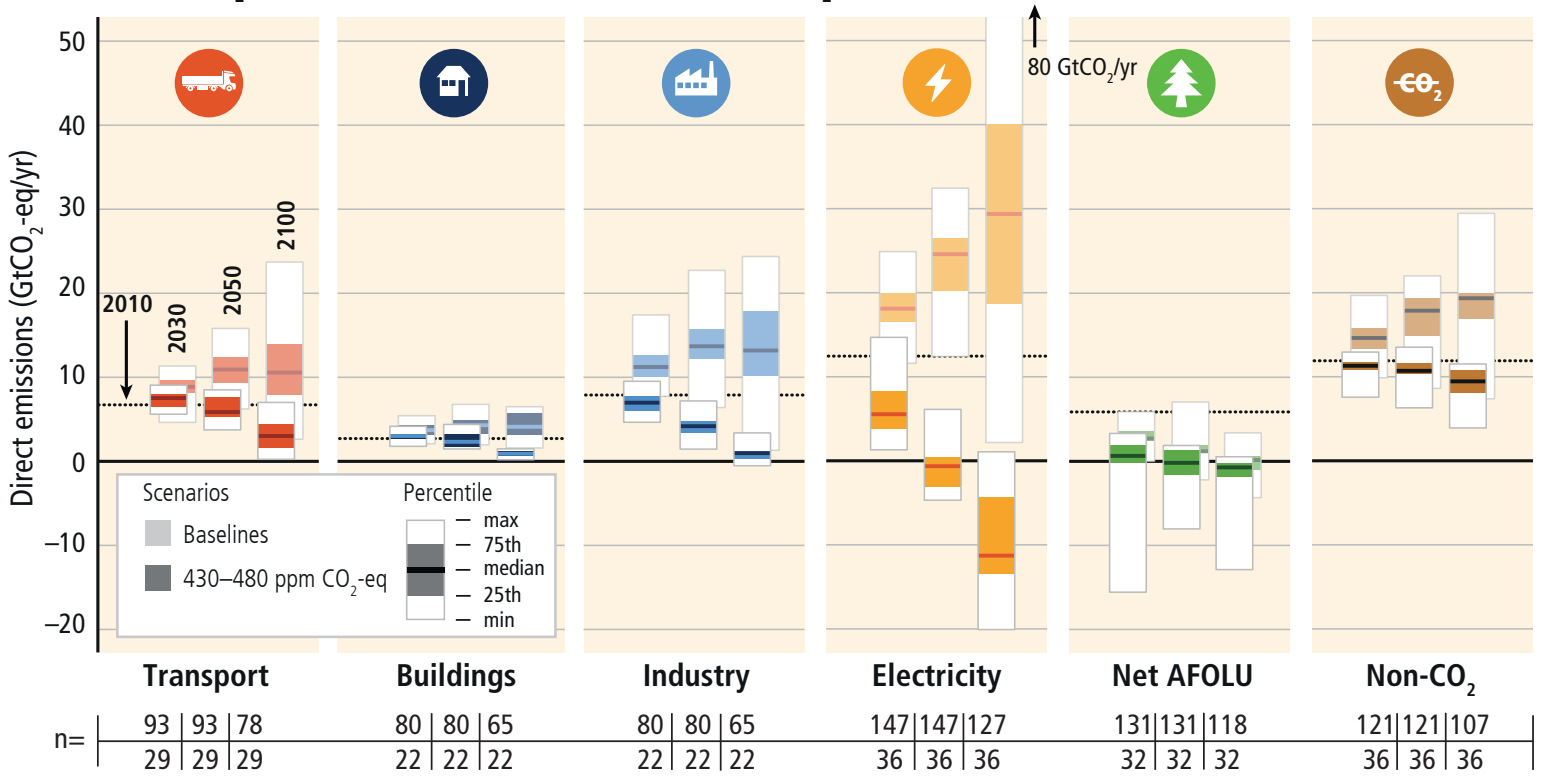

Figure SPM.14 | Carbon dioxide $\left(\mathrm{CO}_{2}\right)$ emissions by sector and total non- $\mathrm{CO}_{2}$ greenhouse gases (Kyoto gases) across sectors in baseline (faded bars) and mitigation scenarios (solid colour bars) that reach about 450 (430 to 480) ppm $\mathrm{CO}_{2}$-eq concentrations in 2100 (likely to limit warming to $2^{\circ} \mathrm{C}$ above preindustrial levels). Mitigation in the end-use sectors leads also to indirect emissions reductions in the upstream energy supply sector. Direct emissions of the end-use sectors thus do not include the emission reduction potential at the supply-side due to, for example, reduced electricity demand. The numbers at the bottom of the graphs refer to the number of scenarios included in the range (upper row: baseline scenarios; lower row: mitigation scenarios), which differs across sectors and time due to different sectoral resolution and time horizon of models. Emissions ranges for mitigation scenarios include the full portfolio of mitigation options; many models cannot reach $450 \mathrm{ppm} \mathrm{CO}$-eq concentration by 2100 in the absence of carbon dioxide capture and storage (CCS). Negative emissions in the electricity sector are due to the application of bioenergy with carbon dioxide capture and storage (BECCS). 'Net' agriculture, forestry and other land use (AFOLU) emissions consider afforestation, reforestation as well as deforestation activities. $\{4.3$, Figure 4.1$\}$ 
Near-term reductions in energy demand are an important element of cost-effective mitigation strategies, provide more flexibility for reducing carbon intensity in the energy supply sector, hedge against related supply-side risks, avoid lock-in to carbon-intensive infrastructures, and are associated with important co-benefits. The most cost-effective mitigation options in forestry are afforestation, sustainable forest management and reducing deforestation, with large differences in their relative importance across regions; and in agriculture, cropland management, grazing land management and restoration of organic soils (medium evidence, high agreement). \{4.3, Figures 4.1, 4.2, Table 4.3\}

Behaviour, lifestyle and culture have a considerable influence on energy use and associated emissions, with high mitigation potential in some sectors, in particular when complementing technological and structural change (medium evidence, medium agreement). Emissions can be substantially lowered through changes in consumption patterns, adoption of energy savings measures, dietary change and reduction in food wastes. $\{4.1,4.3\}$

\section{SPM 4.4 Policy approaches for adaptation and mitigation, technology and finance}

Effective adaptation and mitigation responses will depend on policies and measures across multiple scales: international, regional, national and sub-national. Policies across all scales supporting technology development, diffusion and transfer, as well as finance for responses to climate change, can complement and enhance the effectiveness of policies that directly promote adaptation and mitigation. $\{4.4\}$

International cooperation is critical for effective mitigation, even though mitigation can also have local co-benefits. Adaptation focuses primarily on local to national scale outcomes, but its effectiveness can be enhanced through coordination across governance scales, including international cooperation: $\{3.1,4.4 .1\}$

- The United Nations Framework Convention on Climate Change (UNFCCC) is the main multilateral forum focused on addressing climate change, with nearly universal participation. Other institutions organized at different levels of governance have resulted in diversifying international climate change cooperation. $\{4.4 .1\}$

- The Kyoto Protocol offers lessons towards achieving the ultimate objective of the UNFCCC, particularly with respect to participation, implementation, flexibility mechanisms and environmental effectiveness (medium evidence, low agreement). $\{4.4 .1\}$

- Policy linkages among regional, national and sub-national climate policies offer potential climate change mitigation benefits (medium evidence, medium agreement). Potential advantages include lower mitigation costs, decreased emission leakage and increased market liquidity. $\{4.4 .1\}$

- International cooperation for supporting adaptation planning and implementation has received less attention historically than mitigation but is increasing and has assisted in the creation of adaptation strategies, plans and actions at the national, sub-national and local level (high confidence). $\{4.4 .1\}$

There has been a considerable increase in national and sub-national plans and strategies on both adaptation and mitigation since the AR4, with an increased focus on policies designed to integrate multiple objectives, increase co-benefits and reduce adverse side effects (high confidence): $\{4.4 .2 .1,4.4 .2 .2\}$

- National governments play key roles in adaptation planning and implementation (robust evidence, high agreement) through coordinating actions and providing frameworks and support. While local government and the private sector have different functions, which vary regionally, they are increasingly recognized as critical to progress in adaptation, given their roles in scaling up adaptation of communities, households and civil society and in managing risk information and financing (medium evidence, high agreement). \{4.4.2.1\}

- Institutional dimensions of adaptation governance, including the integration of adaptation into planning and decisionmaking, play a key role in promoting the transition from planning to implementation of adaptation (robust evidence, 
high agreement). Examples of institutional approaches to adaptation involving multiple actors include economic options (e.g., insurance, public-private partnerships), laws and regulations (e.g., land-zoning laws) and national and government policies and programmes (e.g., economic diversification). \{4.2, 4.4.2.1, Table SPM.3\}

- In principle, mechanisms that set a carbon price, including cap and trade systems and carbon taxes, can achieve mitigation in a cost-effective way but have been implemented with diverse effects due in part to national circumstances as well as policy design. The short-run effects of cap and trade systems have been limited as a result of loose caps or caps that have not proved to be constraining (limited evidence, medium agreement). In some countries, tax-based policies specifically aimed at reducing GHG emissions_-alongside technology and other policies—have helped to weaken the link between GHG emissions and GDP (high confidence). In addition, in a large group of countries, fuel taxes (although not necessarily designed for the purpose of mitigation) have had effects that are akin to sectoral carbon taxes. \{4.4.2.2\}

- Regulatory approaches and information measures are widely used and are often environmentally effective (medium evidence, medium agreement). Examples of regulatory approaches include energy efficiency standards; examples of information programmes include labelling programmes that can help consumers make better-informed decisions. \{4.4.2.2\}

- Sector-specific mitigation policies have been more widely used than economy-wide policies (medium evidence, high agreement). Sector-specific policies may be better suited to address sector-specific barriers or market failures and may be bundled in packages of complementary policies. Although theoretically more cost-effective, administrative and political barriers may make economy-wide policies harder to implement. Interactions between or among mitigation policies may be synergistic or may have no additive effect on reducing emissions. \{4.4.2.2\}

- Economic instruments in the form of subsidies may be applied across sectors, and include a variety of policy designs, such as tax rebates or exemptions, grants, loans and credit lines. An increasing number and variety of renewable energy (RE) policies including subsidies - motivated by many factors - have driven escalated growth of RE technologies in recent years. At the same time, reducing subsidies for GHG-related activities in various sectors can achieve emission reductions, depending on the social and economic context (high confidence). \{4.4.2.2\}

Co-benefits and adverse side effects of mitigation could affect achievement of other objectives such as those related to human health, food security, biodiversity, local environmental quality, energy access, livelihoods and equitable sustainable development. The potential for co-benefits for energy end-use measures outweighs the potential for adverse side effects whereas the evidence suggests this may not be the case for all energy supply and agriculture, forestry and other land use (AFOLU) measures. Some mitigation policies raise the prices for some energy services and could hamper the ability of societies to expand access to modern energy services to underserved populations (low confidence). These potential adverse side effects on energy access can be avoided with the adoption of complementary policies such as income tax rebates or other benefit transfer mechanisms (medium confidence). Whether or not side effects materialize, and to what extent side effects materialize, will be case- and site-specific, and depend on local circumstances and the scale, scope and pace of implementation. Many co-benefits and adverse side effects have not been well-quantified. \{4.3, 4.4.2.2, Box 3.4\}

Technology policy (development, diffusion and transfer) complements other mitigation policies across all scales, from international to sub-national; many adaptation efforts also critically rely on diffusion and transfer of technologies and management practices (high confidence). Policies exist to address market failures in R\&D, but the effective use of technologies can also depend on capacities to adopt technologies appropriate to local circumstances. \{4.4.3\}

Substantial reductions in emissions would require large changes in investment patterns (high confidence). For mitigation scenarios that stabilize concentrations (without overshoot) in the range of 430 to $530 \mathrm{ppm} \mathrm{CO} \mathrm{C}_{2}$-eq by $210{ }^{19}$, annual investments in low carbon electricity supply and energy efficiency in key sectors (transport, industry and buildings) are projected in the scenarios to rise by several hundred billion dollars per year before 2030. Within appropriate enabling environments, the private sector, along with the public sector, can play important roles in financing mitigation and adaptation (medium evidence, high agreement). $\{4.4 .4\}$

19 This range comprises scenarios that reach 430 to $480 \mathrm{ppm} \mathrm{CO}$-eq by 2100 (likely to limit warming to $2^{\circ} \mathrm{C}$ above pre-industrial levels) and scenarios that reach 480 to $530 \mathrm{ppm} \mathrm{CO}_{2}$-eq by 2100 (without overshoot: more likely than not to limit warming to $2^{\circ} \mathrm{C}$ above pre-industrial levels). 
Financial resources for adaptation have become available more slowly than for mitigation in both developed and developing countries. Limited evidence indicates that there is a gap between global adaptation needs and the funds available for adaptation (medium confidence). There is a need for better assessment of global adaptation costs, funding and investment. Potential synergies between international finance for disaster risk management and adaptation have not yet been fully realized (high confidence). $\{4.4 .4\}$

\section{SPM 4.5 Trade-offs, synergies and interactions with sustainable development}

Climate change is a threat to sustainable development. Nonetheless, there are many opportunities to link mitigation, adaptation and the pursuit of other societal objectives through integrated responses (high confidence). Successful implementation relies on relevant tools, suitable governance structures and enhanced capacity to respond (medium confidence). $\{3.5,4.5\}$

Climate change exacerbates other threats to social and natural systems, placing additional burdens particularly on the poor (high confidence). Aligning climate policy with sustainable development requires attention to both adaptation and mitigation (high confidence). Delaying global mitigation actions may reduce options for climate-resilient pathways and adaptation in the future. Opportunities to take advantage of positive synergies between adaptation and mitigation may decrease with time, particularly if limits to adaptation are exceeded. Increasing efforts to mitigate and adapt to climate change imply an increasing complexity of interactions, encompassing connections among human health, water, energy, land use and biodiversity (medium evidence, high agreement). $\{3.1,3.5,4.5\}$

Strategies and actions can be pursued now which will move towards climate-resilient pathways for sustainable development, while at the same time helping to improve livelihoods, social and economic well-being and effective environmental management. In some cases, economic diversification can be an important element of such strategies. The effectiveness of integrated responses can be enhanced by relevant tools, suitable governance structures and adequate institutional and human capacity (medium confidence). Integrated responses are especially relevant to energy planning and implementation; interactions among water, food, energy and biological carbon sequestration; and urban planning, which provides substantial opportunities for enhanced resilience, reduced emissions and more sustainable development (medium confidence). $\{3.5,4.4,4.5\}$ 



\section{Climate Change 2014}

Synthesis Report 



\section{Introduction}




\section{Introduction}

The Synthesis Report (SYR) of the IPCC Fifth Assessment Report (AR5) provides an overview of the state of knowledge concerning the science of climate change, emphasizing new results since the publication of the IPCC Fourth Assessment Report (AR4) in 2007. The SYR synthesizes the main findings of the AR5 based on contributions from Working Group I (The Physical Science Basis), Working Group II (Impacts, Adaptation and Vulnerability) and Working Group III (Mitigation of Climate Change), plus two additional IPCC reports (Special Report on Renewable Energy Sources and Climate Change Mitigation and Special Report on Managing the Risks of Extreme Events and Disasters to Advance Climate Change Adaptation).

The AR5 SYR longer report is divided into four topics. Topic 1 (Observed Changes and their Causes) focuses on observational evidence for a changing climate, the impacts caused by this change and the human contributions to it. Topic 2 (Future Climate Changes, Risks and Impacts) assesses projections of future climate change and the resultant projected impacts and risks. Topic 3 (Future Pathways for Adaptation, Mitigation and Sustainable Development) considers adaptation and mitigation as complementary strategies for reducing and managing the risks of climate change. Topic 4 (Adaptation and Mitigation) describes individual adaptation and mitigation options and policy approaches. It also addresses integrated responses that link mitigation and adaptation with other societal objectives.

The challenges of understanding and managing risks and uncertainties are important themes in this report. See Box 1 (Risk and the Management of an Uncertain Future) and Box 2 (Communicating the Degree of Certainty in Assessment Findings).

This report includes information relevant to Article 2 of the United Nations Framework Convention on Climate Change (UNFCCC).

\section{Box Introduction.1 | Risk and the Management of an Uncertain Future}

Climate change exposes people, societies, economic sectors and ecosystems to risk. Risk is the potential for consequences when something of value is at stake and the outcome is uncertain, recognizing the diversity of values. \{WGII SPM Background Box SPM.2, WGIII 2.1, SYR Glossary\}

Risks from climate change impacts arise from the interaction between hazard (triggered by an event or trend related to climate change), vulnerability (susceptibility to harm) and exposure (people, assets or ecosystems at risk). Hazards include processes that range from brief events, such as severe storms, to slow trends, such as multi-decade droughts or multi-century sea level rise. Vulnerability and exposure are both sensitive to a wide range of social and economic processes, with possible increases or decreases depending on development pathways. Risks and co-benefits also arise from policies that aim to mitigate climate change or to adapt to it. (1.5)

Risk is often represented as the probability of occurrence of hazardous events or trends multiplied by the magnitude of the consequences if these events occur. Therefore, high risk can result not only from high probability outcomes but also from low probability outcomes with very severe consequences. This makes it important to assess the full range of possible outcomes, from low probability tail outcomes to very likely outcomes. For example, it is unlikely that global mean sea level will rise by more than one meter in this century, but the consequence of a greater rise could be so severe that this possibility becomes a significant part of risk assessment. Similarly, low confidence but high consequence outcomes are also policy relevant; for instance the possibility that the response of Amazon forest could substantially amplify climate change merits consideration despite our currently imperfect ability to project the outcome. (2.4, Table 2.3) \{WGI Table 13.5, WGII SPM A-3, 4.4, Box 4-3, WGIII Box 3-9, SYR Glossary\}

Risk can be understood either qualitatively or quantitatively. It can be reduced and managed using a wide range of formal or informal tools and approaches that are often iterative. Useful approaches for managing risk do not necessarily require that risk levels can be accurately quantified. Approaches recognizing diverse qualitative values, goals and priorities, based on ethical, psychological, cultural or social factors, could increase the effectiveness of risk management. \{WGII 1.1.2, 2.4, 2.5, 19.3, WGIII 2.4, 2.5, 3.4\} 


\section{Box Introduction.2 | Communicating the Degree of Certainty in Assessment Findings}

An integral feature of IPCC reports is the communication of the strength of and uncertainties in scientific understanding underlying assessment findings. Uncertainty can result from a wide range of sources. Uncertainties in the past and present are the result of limitations of available measurements, especially for rare events, and the challenges of evaluating causation in complex or multi-component processes that can span physical, biological and human systems. For the future, climate change involves changing likelihoods of diverse outcomes. Many processes and mechanisms are well understood, but others are not. Complex interactions among multiple climatic and non-climatic influences changing over time lead to persistent uncertainties, which in turn lead to the possibility of surprises. Compared to past IPCC reports, the AR5 assesses a substantially larger knowledge base of scientific, technical and socio-economic literature. $\{W G I 1.4$, WGII SPM A-3, 1.1.2, WGIII 2.3\}

The IPCC Guidance Note on Uncertainty a defines a common approach to evaluating and communicating the degree of certainty in findings of the assessment process. Each finding is grounded in an evaluation of underlying evidence and agreement. In many cases, a synthesis of evidence and agreement supports an assignment of confidence, especially for findings with stronger agreement and multiple independent lines of evidence. The degree of certainty in each key finding of the assessment is based on the type, amount, quality and consistency of evidence (e.g., data, mechanistic understanding, theory, models, expert judgment) and the degree of agreement. The summary terms for evidence are: limited, medium or robust. For agreement, they are low, medium or high. Levels of confidence include five qualifiers: very low, low, medium, high and very high, and are typeset in italics, e.g., medium confidence. The likelihood, or probability, of some well-defined outcome having occurred or occurring in the future can be described quantitatively through the following terms: virtually certain, 99-100\% probability; extremely likely, 95-100\%; very likely, 90-100\%; likely, 66-100\%; more likely than not, >50-100\%; about as likely as not, 33-66\%; unlikely, 0-33\%; very unlikely, 0-10\%; extremely unlikely, 0-5\%; and exceptionally unlikely, 0-1\%. Additional terms (extremely likely, 95-100\%; more likely than not, $>50-100 \%$; more unlikely than likely, $0-<50 \%$; and extremely unlikely, 0-5\%) may also be used when appropriate. Assessed likelihood is typeset in italics, e.g., very likely. Unless otherwise indicated, findings assigned a likelihood term are associated with high or very high confidence. Where appropriate, findings are also formulated as statements of fact without using uncertainty qualifiers. \{WGI SPM B, WGII Background Box SPM.3, WGIII 2.1\}

\footnotetext{
a Mastrandrea, M.D., C.B. Field, T.F. Stocker, O. Edenhofer, K.L. Ebi, D.J. Frame, H. Held, E. Kriegler, K.J. Mach, P.R. Matschoss, G.-K. Plattner, G.W. Yohe and F.W. Zwiers, 2010: Guidance Note for Lead Authors of the IPCC Fifth Assessment Report on Consistent Treatment of Uncertainties. Intergovernmental Panel on Climate Change (IPCC), Geneva, Switzerland, 4 pp.
} 



\section{Observed Changes and their Causes}




\section{Topic 1: Observed Changes and their Causes}

Human influence on the climate system is clear, and recent anthropogenic emissions of greenhouse gases are the highest in history. Recent climate changes have had widespread impacts on human and natural systems.

Topic 1 focuses on observational evidence of a changing climate, the impacts caused by this change and the human contributions to it. It discusses observed changes in climate (1.1) and external influences on climate (forcings), differentiating those forcings that are of anthropogenic origin, and their contributions by economic sectors and greenhouse gases (GHGs) (1.2). Section 1.3 attributes observed climate change to its causes and attributes impacts on human and natural systems to climate change, determining the degree to which those impacts can be attributed to climate change. The changing probability of extreme events and their causes are discussed in Section 1.4, followed by an account of exposure and vulnerability within a risk context (1.5) and a section on adaptation and mitigation experience (1.6).

\subsection{Observed changes in the climate system}

Warming of the climate system is unequivocal, and since the 1950s, many of the observed changes are unprecedented over decades to millennia. The atmosphere and ocean have warmed, the amounts of snow and ice have diminished, and sea level has risen.

\subsubsection{Atmosphere}

Each of the last three decades has been successively warmer at the Earth's surface than any preceding decade since 1850. The period from 1983 to 2012 was very likely the warmest 30-year period of the last 800 years in the Northern Hemisphere, where such assessment is possible (high confidence) and likely the warmest 30-year period of the last 1400 years (medium confidence). $\{W G I$ 2.4.3, 5.3.5\}

The globally averaged combined land and ocean surface temperature data as calculated by a linear trend show a warming of 0.85 [0.65 to 1.06$]^{\circ}{ }^{\circ}{ }^{20}$ over the period 1880 to 2012 , for which multiple independently produced datasets exist. The total increase between the average of the 1850-1900 period and the 2003-2012 period is 0.78 [0.72 to 0.85$]^{\circ} \mathrm{C}$, based on the single longest dataset available. For the longest period when calculation of regional trends is sufficiently complete (1901 to 2012), almost the entire globe has experienced surface warming (Figure 1.1). \{WGI SPM B.1, 2.4.3\}

In addition to robust multi-decadal warming, the globally averaged surface temperature exhibits substantial decadal and interannual variability (Figure 1.1). Due to this natural variability, trends based on short records are very sensitive to the beginning and end dates and do not in general reflect long-term climate trends. As one example, the rate of warming over the past 15 years (1998-2012; 0.05 [-0.05 to 0.15] ${ }^{\circ} \mathrm{C}$ per decade), which begins with a strong El Niño, is smaller than the rate calculated since $1951(1951-2012 ; 0.12 \text { [0.08 to } 0.14]^{\circ} \mathrm{C}$ per decade; see Box 1.1). \{WGI SPM B.1, 2.4.3\}
Based on multiple independent analyses of measurements, it is virtually certain that globally the troposphere has warmed and the lower stratosphere has cooled since the mid-20th century. There is medium confidence in the rate of change and its vertical structure in the Northern Hemisphere extratropical troposphere. \{WGI SPM B.1, 2.4.4\}

Confidence in precipitation change averaged over global land areas since 1901 is low prior to 1951 and medium afterwards. Averaged over the mid-latitude land areas of the Northern Hemisphere, precipitation has likely increased since 1901 (medium confidence before and high confidence after 1951). For other latitudes area-averaged long-term positive or negative trends have low confidence (Figure 1.1). \{WGI SPM B.1, Figure SPM.2, 2.5.1\}

\subsubsection{Ocean}

Ocean warming dominates the increase in energy stored in the climate system, accounting for more than $90 \%$ of the energy accumulated between 1971 and 2010 (high confidence) with only about $1 \%$ stored in the atmosphere (Figure 1.2). On a global scale, the ocean warming is largest near the surface, and the upper $75 \mathrm{~m}$ warmed by 0.11 [0.09 to 0.13 ] ${ }^{\circ} \mathrm{C}$ per decade over the period 1971 to 2010 . It is virtually certain that the upper ocean (0-700 m) warmed from 1971 to 2010, and it likely warmed between the 1870s and 1971. It is likely that the ocean warmed from 700 to $2000 \mathrm{~m}$ from 1957 to 2009 and from $3000 \mathrm{~m}$ to the bottom for the period 1992 to 2005 (Figure 1.2). \{WGI SPM B.2, 3.2, Box 3.1\}

It is very likely that regions of high surface salinity, where evaporation dominates, have become more saline, while regions of low salinity, where precipitation dominates, have become fresher since the 1950s. These regional trends in ocean salinity provide indirect evidence for changes in evaporation and precipitation over the oceans and thus for changes in the global water cycle (medium confidence). There is no observational evidence of a long-term trend in the Atlantic Meridional Overturning Circulation (AMOC). \{WGI SPM B.2, 2.5, 3.3, 3.4.3, 3.5, 3.6.3\} 20 Ranges in square brackets indicate a $90 \%$ uncertainty interval unless otherwise stated. The $90 \%$ uncertainty interval is expected to have a $90 \%$ likelihood of covering the value
that is being estimated. Uncertainty intervals are not necessarily symmetric about the corresponding best estimate. A best estimate of that value is also given where available. 
(a) Observed globally averaged combined land and ocean surface temperature anomaly 1850-2012

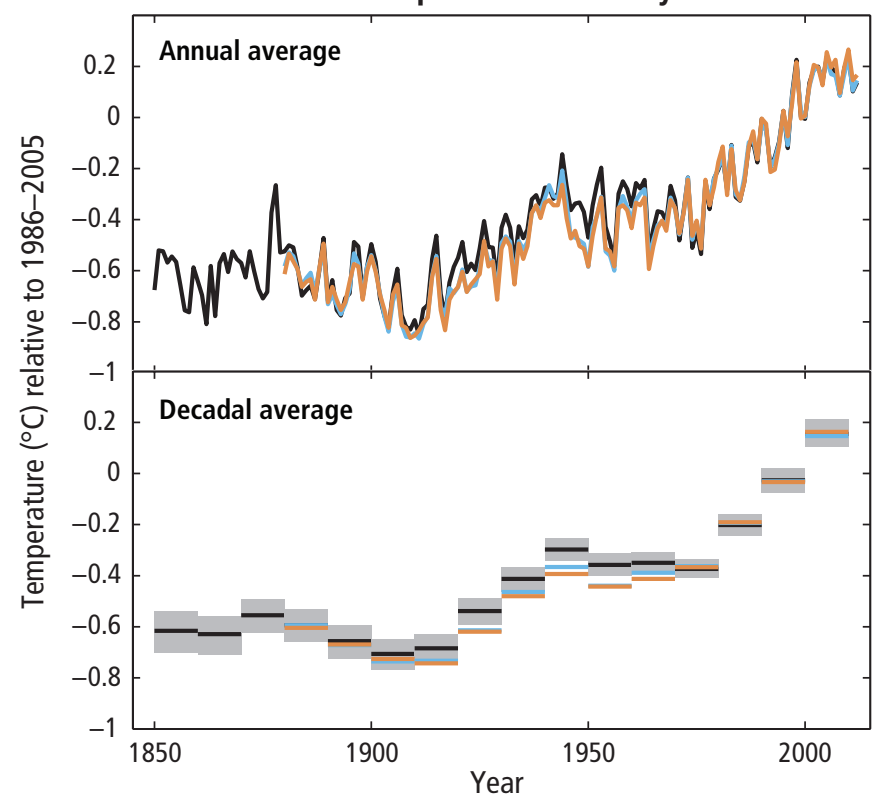

(b)

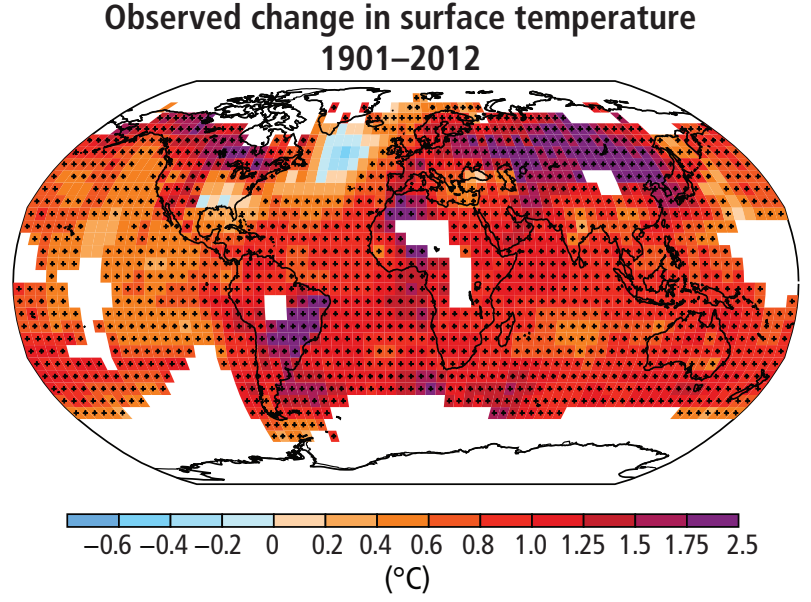

(c)

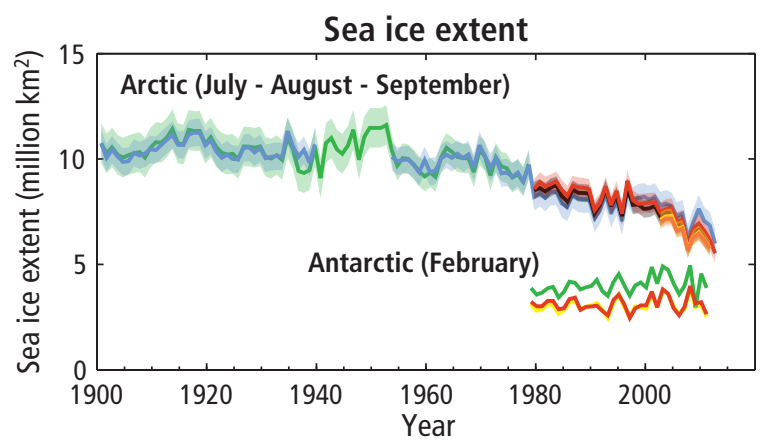

(d) Global mean sea level change 1900-2010

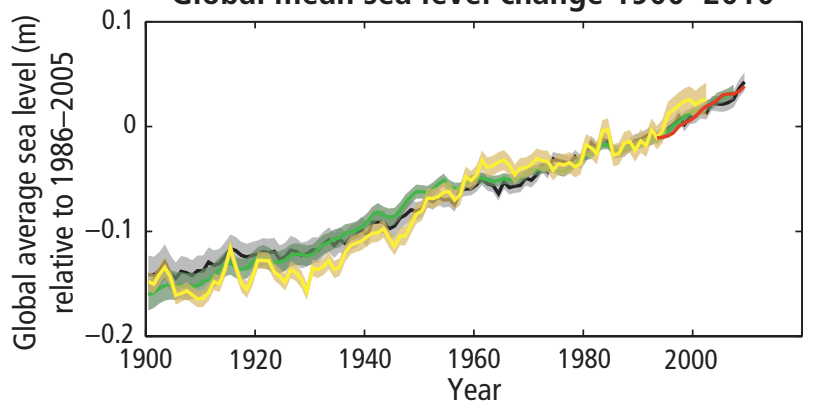

(e)

Observed change in annual precipitation over land

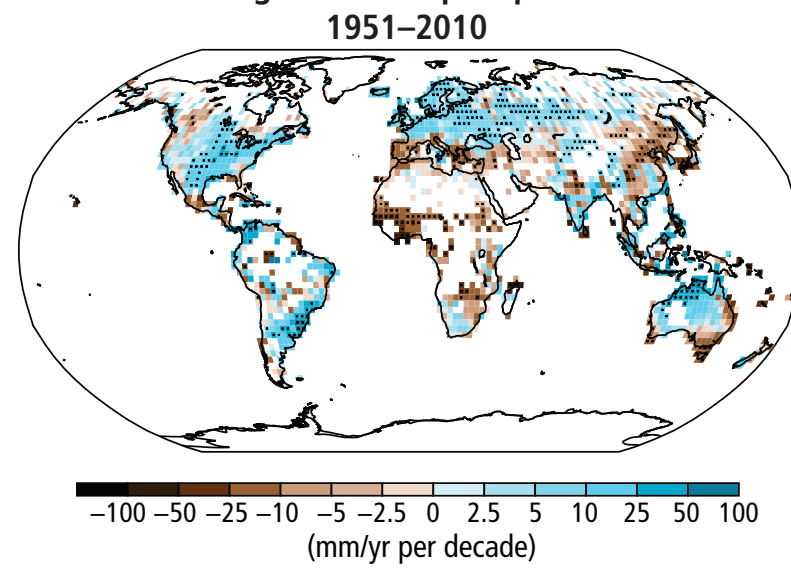

Figure 1.1 | Multiple observed indicators of a changing global climate system. (a) Observed globally averaged combined land and ocean surface temperature anomalies (relative to the mean of 1986 to 2005 period, as annual and decadal averages) with an estimate of decadal mean uncertainty included for one data set (grey shading). \{WGI Figure SPM. 1, Figure 2.20; a listing of data sets and further technical details are given in the WGI Technical Summary Supplementary Material WGI TS.SM.1.1\} (b) Map of the observed surface temperature change, from 1901 to 2012, derived from temperature trends determined by linear regression from one data set (orange line in Panel a). Trends have been calculated where data availability permitted a robust estimate (i.e., only for grid boxes with greater than $70 \%$ complete records and more than $20 \%$ data availability in the first and last $10 \%$ of the time period), other areas are white. Grid boxes where the trend is significant, at the 10\% level, are indicated by a + sign. \{WGI Figure SPM.1, Figure 2.21, Figure TS.2; a listing of data sets and further technical details are given in the WGI Technical Summary Supplementary Material WGI TS.SM.1.2\} (c) Arctic (July to September average) and Antarctic (February) sea ice extent. (WGI Figure SPM.3, Figure 4.3, Figure 4.SM.2; a listing of data sets and further technical details are given in the WGI Technical Summary Supplementary Material WGI TS.SM.3.2\}. (d) Global mean sea level relative to the 1986-2005 mean of the longest running data set, and with all data sets aligned to have the same value in 1993, the first year of satellite altimetry data. All time series (coloured lines indicating different data sets) show annual values, and where assessed, uncertainties are indicated by coloured shading. \{WGI Figure SPM.3, Figure 3.13; a listing of data sets and further technical details are given in the WGI Technical Summary Supplementary Material WGI TS.SM.3.4\}. (e) Map of observed precipitation change, from 1951 to 2010; trends in annual accumulation calculated using the same criteria as in Panel b. \{WGI Figure SPM.2, TS TFE.1, Figure 2, Figure 2.29. A listing of data sets and further technical details are given in the WGI Technical Summary Supplementary Material WGI TS.SM.2.1\}

Since the beginning of the industrial era, oceanic uptake of $\mathrm{CO}_{2}$ has resulted in acidification of the ocean; the $\mathrm{pH}$ of ocean surface water has decreased by 0.1 (high confidence), corresponding to a $26 \%$ increase in acidity, measured as hydrogen ion concentration. There is medium confidence that, in parallel to warming, oxygen concentrations have decreased in coastal waters and in the open ocean thermocline in many ocean regions since the 1960s, with a likely expansion of tropical oxygen minimum zones in recent decades. \{WGI SPM B.5, TS2.8.5, 3.8.1, 3.8.2, 3.8.3, 3.8.5, Figure 3.20\} 
Energy accumulation within the Earth's climate system

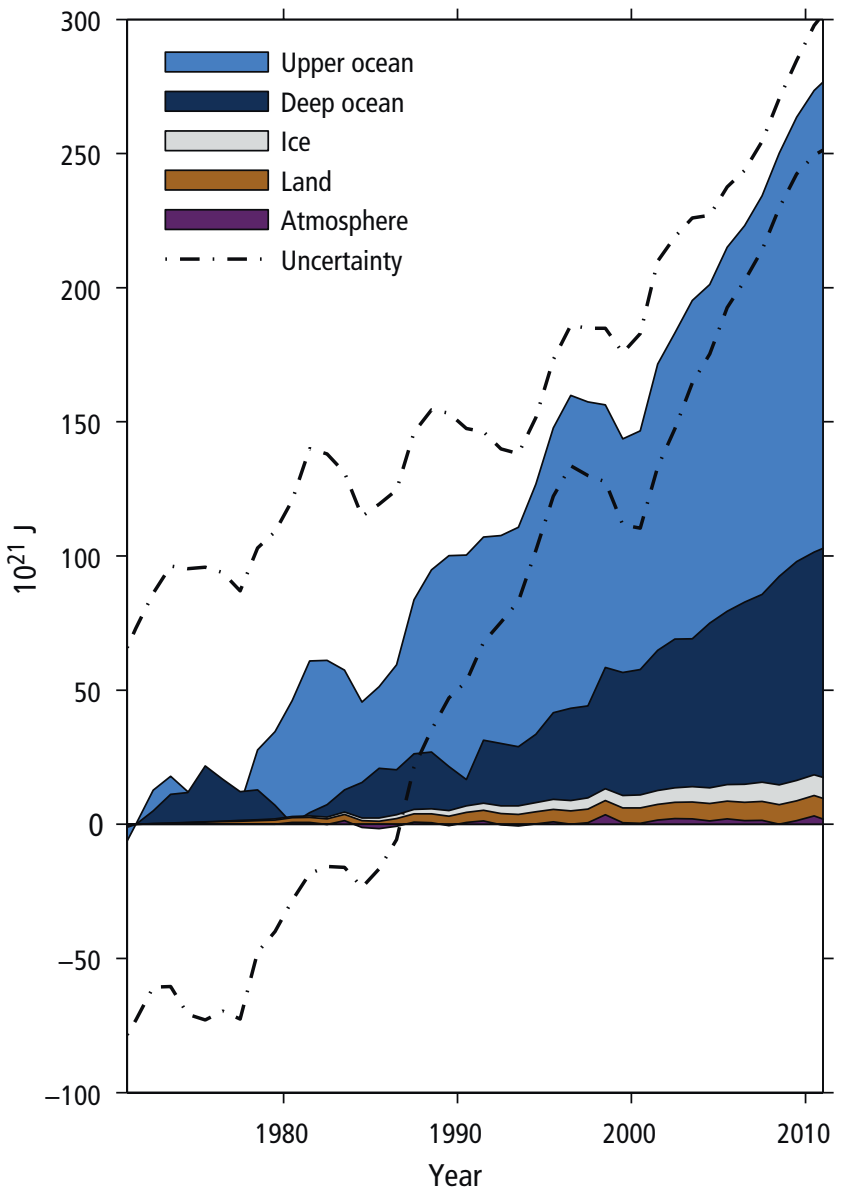

Figure 1.2 Energy accumulation within the Earth's climate system. Estimates are in $10^{21} \mathrm{~J}$, and are given relative to 1971 and from 1971 to 2010, unless otherwise indicated. Components included are upper ocean (above $700 \mathrm{~m}$ ), deep ocean (below 700 m; including below 2000 m estimates starting from 1992), ice melt (for glaciers and ice caps, Greenland and Antarctic ice sheet estimates starting from 1992, and Arctic sea ice estimate from 1979 to 2008), continental (land) warming, and atmospheric warming (estimate starting from 1979). Uncertainty is estimated as error from all five components at $90 \%$ confidence intervals. \{WGI Box 3.1, Figure 1\}

\subsubsection{Cryosphere}

Over the last two decades, the Greenland and Antarctic ice sheets have been losing mass (high confidence). Glaciers have continued to shrink almost worldwide (high confidence). Northern Hemisphere spring snow cover has continued to decrease in extent (high confidence). There is high confidence that there are strong regional differences in the trend in Antarctic sea ice extent, with a very likely increase in total extent. \{WGI SPM B.3, 4.2-4.7\}

Glaciers have lost mass and contributed to sea level rise throughout the 20th century. The rate of ice mass loss from the Greenland ice sheet has very likely substantially increased over the period 1992 to 2011, resulting in a larger mass loss over 2002 to 2011 than over 1992 to 2011. The rate of ice mass loss from the Antarctic ice sheet, mainly from the northern Antarctic Peninsula and the Amundsen Sea sector of West Antarctica, is also likely larger over 2002 to 2011. \{WGI SPM B.3, SPM B.4, 4.3.3, 4.4.2, 4.4.3\}
The annual mean Arctic sea ice extent decreased over the period 1979 (when satellite observations commenced) to 2012. The rate of decrease was very likely in the range 3.5 to $4.1 \%$ per decade. Arctic sea ice extent has decreased in every season and in every successive decade since 1979 , with the most rapid decrease in decadal mean extent in summer (high confidence). For the summer sea ice minimum, the decrease was very likely in the range of 9.4 to $13.6 \%$ per decade (range of 0.73 to 1.07 million $\mathrm{km}^{2}$ per decade) (see Figure 1.1). It is very likely that the annual mean Antarctic sea ice extent increased in the range of 1.2 to $1.8 \%$ per decade (range of 0.13 to 0.20 million $\mathrm{km}^{2}$ per decade) between 1979 and 2012. However, there is high confidence that there are strong regional differences in Antarctica, with extent increasing in some regions and decreasing in others. \{WGI SPM B.5, 4.2.2, 4.2.3\}

There is very high confidence that the extent of Northern Hemisphere snow cover has decreased since the mid-20th century by $1.6[0.8$ to 2.4] \% per decade for March and April, and $11.7 \%$ per decade for June, over the 1967 to 2012 period. There is high confidence that permafrost temperatures have increased in most regions of the Northern Hemisphere since the early 1980s, with reductions in thickness and areal extent in some regions. The increase in permafrost temperatures has occurred in response to increased surface temperature and changing snow cover. \{WGI SPM B.3, 4.5, 4.7.2\}

\subsubsection{Sea level}

Over the period 1901-2010, global mean sea level rose by 0.19 [0.17 to 0.21$] \mathrm{m}$ (Figure 1.1). The rate of sea level rise since the mid-19th century has been larger than the mean rate during the previous two millennia (high confidence). \{WGI SPM B.4, 3.7.2, 5.6.3, 13.2\}

It is very likely that the mean rate of global averaged sea level rise was 1.7 [1.5 to 1.9] mm/yr between 1901 and 2010 and 3.2 [2.8 to 3.6] $\mathrm{mm} / \mathrm{yr}$ between 1993 and 2010. Tide gauge and satellite altimeter data are consistent regarding the higher rate during the latter period. It is likely that similarly high rates occurred between 1920 and 1950. \{WGI SPM B.4, 3.7, 13.2\}

Since the early 1970s, glacier mass loss and ocean thermal expansion from warming together explain about $75 \%$ of the observed global mean sea level rise (high confidence). Over the period 1993-2010, global mean sea level rise is, with high confidence, consistent with the sum of the observed contributions from ocean thermal expansion, due to warming, from changes in glaciers, the Greenland ice sheet, the Antarctic ice sheet and land water storage. \{WGI SPM B.4, 13.3.6\}

Rates of sea level rise over broad regions can be several times larger or smaller than the global mean sea level rise for periods of several decades, due to fluctuations in ocean circulation. Since 1993, the regional rates for the Western Pacific are up to three times larger than the global mean, while those for much of the Eastern Pacific are near zero or negative. $\{$ WGI 3.7.3, FAQ 13.1\}

There is very high confidence that maximum global mean sea level during the last interglacial period $(129,000$ to 116,000 years ago) was, for several thousand years, at least $5 \mathrm{~m}$ higher than present and 


\section{Box 1.1 | Recent Temperature Trends and their Implications}

The observed reduction in surface warming trend over the period 1998 to 2012 as compared to the period 1951 to 2012, is due in roughly equal measure to a reduced trend in radiative forcing and a cooling contribution from natural internal variability, which includes a possible redistribution of heat within the ocean (medium confidence). The rate of warming of the observed global mean surface temperature over the period from 1998 to 2012 is estimated to be around one-third to one-half of the trend over the period from 1951 to 2012 (Box 1.1, Figures 1a and 1c). Even with this reduction in surface warming trend, the climate system has very likely continued to accumulate heat since 1998 (Figure 1.2) and sea level has continued to rise (Figure 1.1). \{WGI SPM D.1, Box 9.2\}

The radiative forcing of the climate system has continued to increase during the 2000s, as has its largest contributor, the atmospheric concentration of $\mathrm{CO}_{2}$. However, the radiative forcing has been increasing at a lower rate over the period from 1998 to 2011, compared to 1984 to 1998 or 1951 to 2011, due to cooling effects from volcanic eruptions and the cooling phase of the solar cycle over the period from 2000 to 2009. There is, however, low confidence in quantifying the role of the forcing trend in causing the reduction in the rate of surface warming. \{WGI 8.5.2, Box 9.2\}

For the period from 1998 to 2012, 111 of the 114 available climate-model simulations show a surface warming trend larger than the observations (Box 1.1, Figure 1a). There is medium confidence that this difference between models and observations is to a substantial degree caused by natural internal climate variability, which sometimes enhances and sometimes counteracts the long-term externally forced warming trend (compare Box 1.1, Figures 1a and 1b; during the period from 1984 to 1998, most model simulations show a smaller warming trend than observed). Natural internal variability thus diminishes the relevance of short trends for long-term climate change. The difference between models and observations may also contain contributions from inadequacies in the solar, volcanic and aerosol forcings used by the models and, in some models, from an overestimate of the response to increasing greenhouse gas and other anthropogenic forcing (the latter dominated by the effects of aerosols). $\{W G I$ 2.4.3, Box 9.2, 9.4.1, 10.3.1.1\}

For the longer period from 1951 to 2012, simulated surface warming trends are consistent with the observed trend (very high confidence) (Box 1.1, Figure 1c). Furthermore, the independent estimates of radiative forcing, of surface warming and of observed heat storage (the latter available since 1970) combine to give a heat budget for the Earth that is consistent with the assessed likely range of equilibrium climate sensitivity $\left(1.5-4.5^{\circ} \mathrm{C}\right)^{21}$. The record of observed climate change has thus allowed characterization of the basic properties of the climate system that have implications for future warming, including the equilibrium climate sensitivity and the transient climate response (see Topic 2). \{WGI Box 9.2, 10.8.1, 10.8.2, Box 12.2, Box 13.1\}

(a)

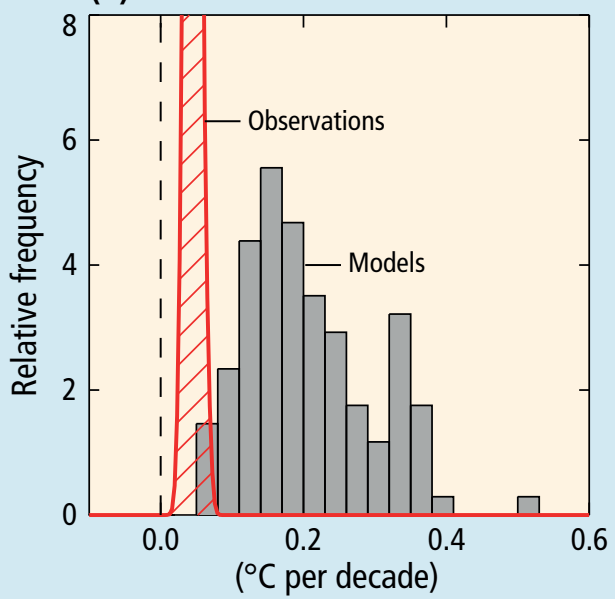

(b)

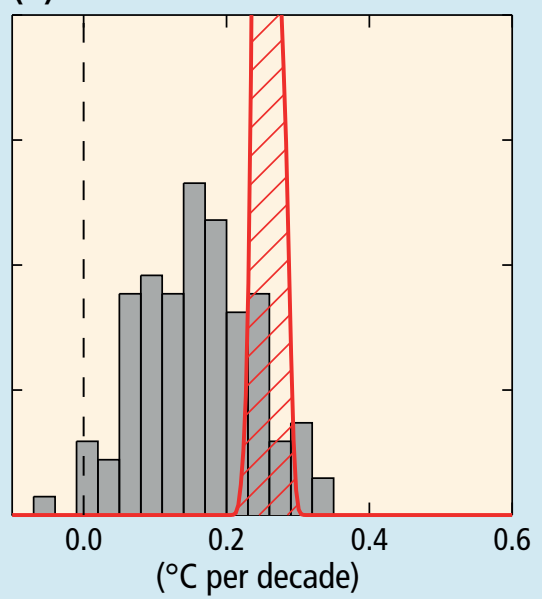

(c)

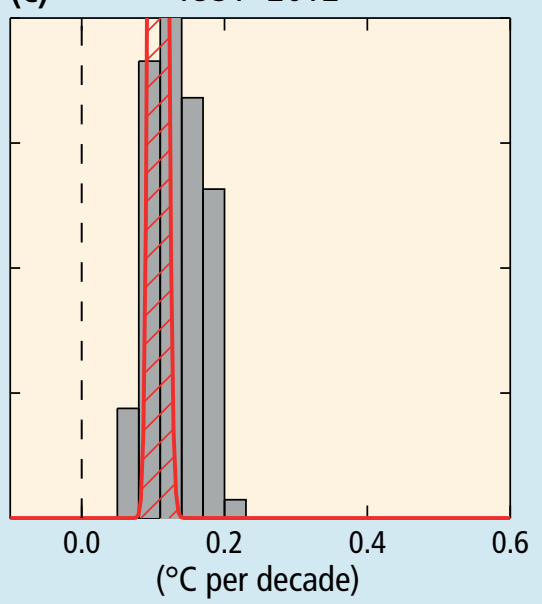

Box 1.1, Figure 1| Trends in the global mean surface temperature over the periods from 1998 to 2012 (a), 1984 to 1998 (b), and 1951 to 2012 (c), from observations (red) and the 114 available simulations with current-generation climate models (grey bars). The height of each grey bar indicates how often a trend of a certain magnitude (in ${ }^{\circ} \mathrm{C}$ per decade) occurs among the 114 simulations. The width of the red-hatched area indicates the statistical uncertainty that arises from constructing a global average from individual station data. This observational uncertainty differs from the one quoted in the text of Section 1.1.1; there, an estimate of natural internal variability is also included. Here, by contrast, the magnitude of natural internal variability is characterised by the spread of the model ensemble. \{based on WGI Box 9.2, Figure 1\}

21 The connection between the heat budget and equilibrium climate sensitivity, which is the long-term surface warming under an assumed doubling of the atmospheric $\mathrm{CO}_{2}$ concentration, arises because a warmer surface causes enhanced radiation to space which counteracts the increase in the Earth's heat content. How much the radiation to space increases for a given increase in surface temperature depends on the same feedback processes (e.g., cloud feedback, water vapour feedback) that determine equilibrium climate sensitivity. 
high confidence that it did not exceed $10 \mathrm{~m}$ above present. During the last interglacial period, the Greenland ice sheet very likely contributed between 1.4 and $4.3 \mathrm{~m}$ to the higher global mean sea level, implying with medium confidence an additional contribution from the Antarctic ice sheet. This change in sea level occurred in the context of different orbital forcing and with high-latitude surface temperature, averaged over several thousand years, at least $2^{\circ} \mathrm{C}$ warmer than present (high confidence). $\{$ WGI SPM B.4, 5.3.4, 5.6.2, 13.2.1\}

\subsection{Past and recent drivers of climate change}

Anthropogenic greenhouse gas emissions have increased since the pre-industrial era driven largely by economic and population growth. From 2000 to 2010 emissions were the highest in history. Historical emissions have driven atmospheric concentrations of carbon dioxide, methane and nitrous oxide to levels that are unprecedented in at least the last 800,000 years, leading to an uptake of energy by the climate system.

Natural and anthropogenic substances and processes that alter the Earth's energy budget are physical drivers of climate change. Radiative forcing quantifies the perturbation of energy into the Earth system caused by these drivers. Radiative forcings larger than zero lead to a near-surface warming, and radiative forcings smaller than zero lead to a cooling. Radiative forcing is estimated based on in-situ and remote observations, properties of GHGs and aerosols, and calculations using numerical models. The radiative forcing over the 1750-2011 period is shown in Figure 1.4 in major groupings. The 'Other Anthropogenic' group is principally comprised of cooling effects from aerosol changes, with smaller contributions from ozone changes, land use reflectance changes and other minor terms. \{WGI SPM C, 8.1, 8.5.1\}

\subsubsection{Natural and anthropogenic radiative forcings}

Atmospheric concentrations of GHGs are at levels that are unprecedented in at least 800,000 years. Concentrations of carbon dioxide $\left(\mathrm{CO}_{2}\right)$, methane $\left(\mathrm{CH}_{4}\right)$ and nitrous oxide $\left(\mathrm{N}_{2} \mathrm{O}\right)$ have all shown large increases since 1750 (40\%, 150\% and 20\%, respectively) (Figure 1.3). $\mathrm{CO}_{2}$ concentrations are increasing at the fastest observed decadal rate of change $(2.0 \pm 0.1 \mathrm{ppm} / \mathrm{yr})$ for $2002-$ 2011. After almost one decade of stable $\mathrm{CH}_{4}$ concentrations since the late 1990s, atmospheric measurements have shown renewed increases since 2007. $\mathrm{N}_{2} \mathrm{O}$ concentrations have steadily increased at a rate of $0.73 \pm 0.03 \mathrm{ppb} / \mathrm{yr}$ over the last three decades. \{WGI SPM B5, 2.2.1, $6.1 .2,6.1 .3,6.3\}$

The total anthropogenic radiative forcing over 1750-2011 is calculated to be a warming effect of 2.3 [1.1 to 3.3$] \mathrm{W} / \mathrm{m}^{2}$ (Figure 1.4), and it has increased more rapidly since 1970 than during prior decades. Carbon dioxide is the largest single contributor to radiative forcing over 1750-2011 and its trend since 1970. The total anthropogenic radiative forcing estimate for 2011 is substantially higher (43\%) than the estimate reported in the IPCC

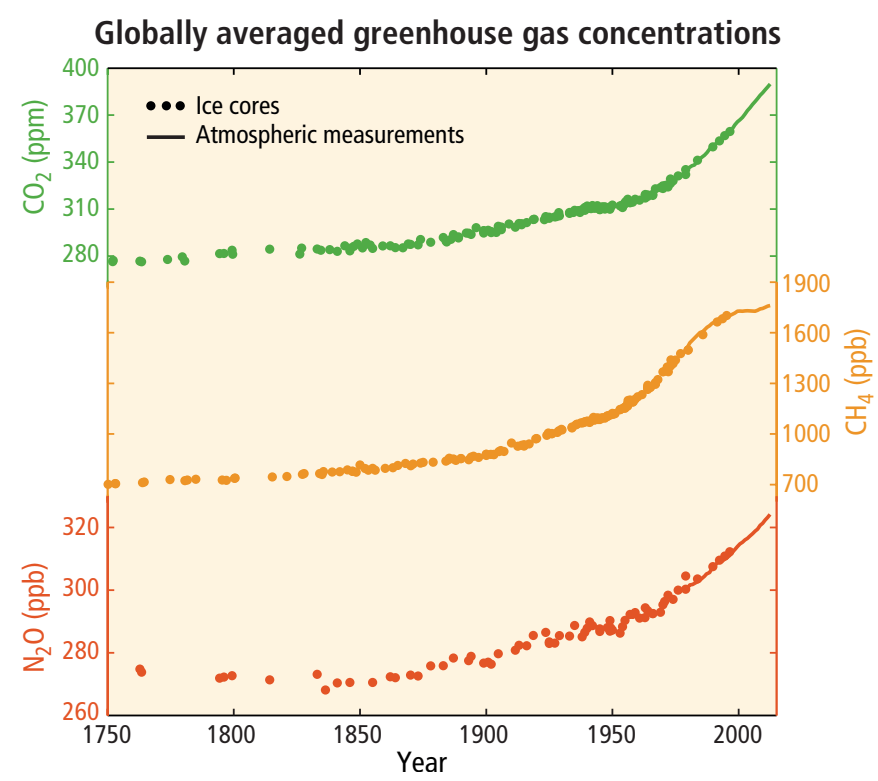

Figure 1.3 | Observed changes in atmospheric greenhouse gas concentrations Atmospheric concentrations of carbon dioxide $\left(\mathrm{CO}_{2}\right.$, green), methane $\left(\mathrm{CH}_{4}\right.$, orange), and nitrous oxide $\left(\mathrm{N}_{2} \mathrm{O}\right.$, red). Data from ice cores (symbols) and direct atmospheric measurements (lines) are overlaid. \{WGI 2.2, 6.2, 6.3, Figure 6.11\}

Fourth Assessment Report (AR4) for the year 2005. This is caused by a combination of continued growth in most GHG concentrations and an improved estimate of radiative forcing from aerosols. \{WGI SPM C, 8.5.1\}

The radiative forcing from aerosols, which includes cloud adjustments, is better understood and indicates a weaker cooling effect than in AR4. The aerosol radiative forcing over $1750-2011$ is estimated as -0.9 [ -1.9 to -0.1$] \mathrm{W} / \mathrm{m}^{2}$ (medium confidence). Radiative forcing from aerosols has two competing components: a dominant cooling effect from most aerosols and their cloud adjustments and a partially offsetting warming contribution from black carbon absorption of solar radiation. There is high confidence that the global mean total aerosol radiative forcing has counteracted a substantial portion of radiative forcing from wellmixed GHGs. Aerosols continue to contribute the largest uncertainty to the total radiative forcing estimate. $\{W G I S P M C, 7.5,8.3,8.5 .1\}$

Changes in solar irradiance and volcanic aerosols cause natural radiative forcing (Figure 1.4). The radiative forcing from stratospheric volcanic aerosols can have a large cooling effect on the climate system for some years after major volcanic eruptions. Changes in total solar irradiance are calculated to have contributed only around $2 \%$ of the total radiative forcing in 2011, relative to 1750 . \{WGI SPM C, Figure SPM.5, 8.4\} 


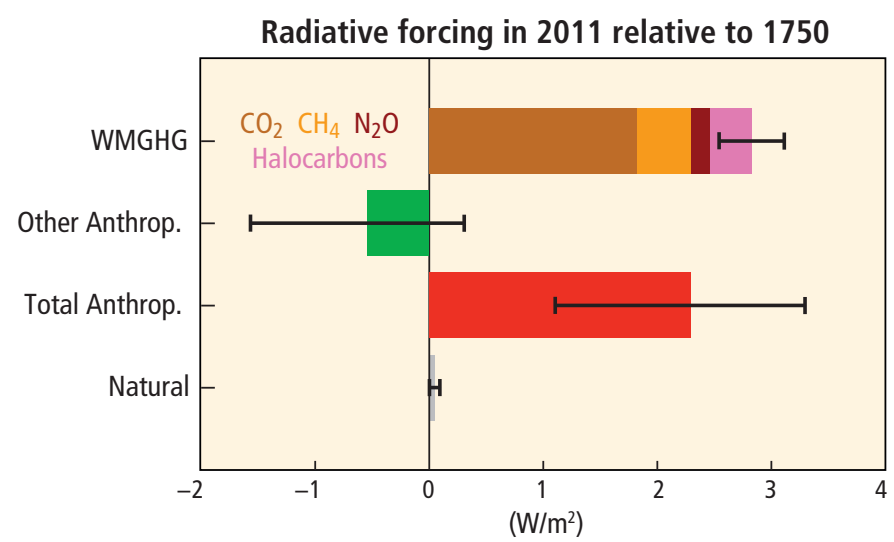

Figure 1.4 | Radiative forcing of climate change during the industrial era (1750-2011). Bars show radiative forcing from well-mixed greenhouse gases (WMGHG), other anthropogenic forcings, total anthropogenic forcings and natural forcings. The error bars indicate the 5 to $95 \%$ uncertainty. Other anthropogenic forcings include aerosol, land use surface reflectance and ozone changes. Natural forcings include solar and volcanic effects. The total anthropogenic radiative forcing for 2011 relative to 1750 is $2.3 \mathrm{~W} / \mathrm{m}^{2}$ (uncertainty range 1.1 to $3.3 \mathrm{~W} / \mathrm{m}^{2}$ ). This corresponds to a $\mathrm{CO}_{2}$-equivalent concentration (see Glossary) of $430 \mathrm{ppm}$ (uncertainty range 340 to 520 ppm). \{Data from WGI 7.5 and Table 8.6\}

\subsubsection{Human activities affecting emission drivers}

About half of the cumulative anthropogenic $\mathrm{CO}_{2}$ emissions between 1750 and 2011 have occurred in the last 40 years (high confidence). Cumulative anthropogenic $\mathrm{CO}_{2}$ emissions of
$2040 \pm 310 \mathrm{GtCO}_{2}$ were added to the atmosphere between 1750 and 2011. Since 1970, cumulative $\mathrm{CO}_{2}$ emissions from fossil fuel combustion, cement production and flaring have tripled, and cumulative $\mathrm{CO}_{2}$ emissions from forestry and other land use (FOLU) ${ }^{22}$ have increased by about $40 \%$ (Figure 1.5) ${ }^{23}$. In 2011, annual $\mathrm{CO}_{2}$ emissions from fossil fuel combustion, cement production and flaring were $34.8 \pm 2.9 \mathrm{GtCO}_{2} / \mathrm{yr}$. For 2002-2011, average annual emissions from FOLU were $3.3 \pm 2.9 \mathrm{GtCO}_{2}$ /yr. $\{W G / 6.3 .1,6.3 .2$, WGIII SPM.3\}

About $40 \%$ of these anthropogenic $\mathrm{CO}_{2}$ emissions have remained in the atmosphere $\left(880 \pm 35 \mathrm{GtCO}_{2}\right)$ since 1750 . The rest was removed from the atmosphere by sinks, and stored in natural carbon cycle reservoirs. Sinks from ocean uptake and vegetation with soils account, in roughly equal measures, for the remainder of the cumulative $\mathrm{CO}_{2}$ emissions. The ocean has absorbed about 30\% of the emitted anthropogenic $\mathrm{CO}_{2}$, causing ocean acidification. $\{W G I 3.8 .1,6.3 .1\}$

Total annual anthropogenic GHG emissions have continued to increase over 1970 to 2010 with larger absolute increases between 2000 and 2010 (high confidence). Despite a growing number of climate change mitigation policies, annual GHG emissions grew on average by $1.0 \mathrm{GtCO}_{2}$-eq (2.2\%) per year, from 2000 to 2010 , compared to $0.4 \mathrm{GtCO}_{2}$-eq (1.3\%) per year, from 1970 to 2000 (Figure 1.6) ) $^{24}$. Total anthropogenic GHG emissions from 2000 to 2010 were the highest in human history and reached $49( \pm 4.5) \mathrm{GtCO}_{2}$-eq/yr in 2010 . The global economic crisis of 2007/2008 reduced emissions only temporarily. \{WGIII SPM.3, 1.3, 5.2, 13.3, 15.2.2, Box TS.5, Figure 15.1\}
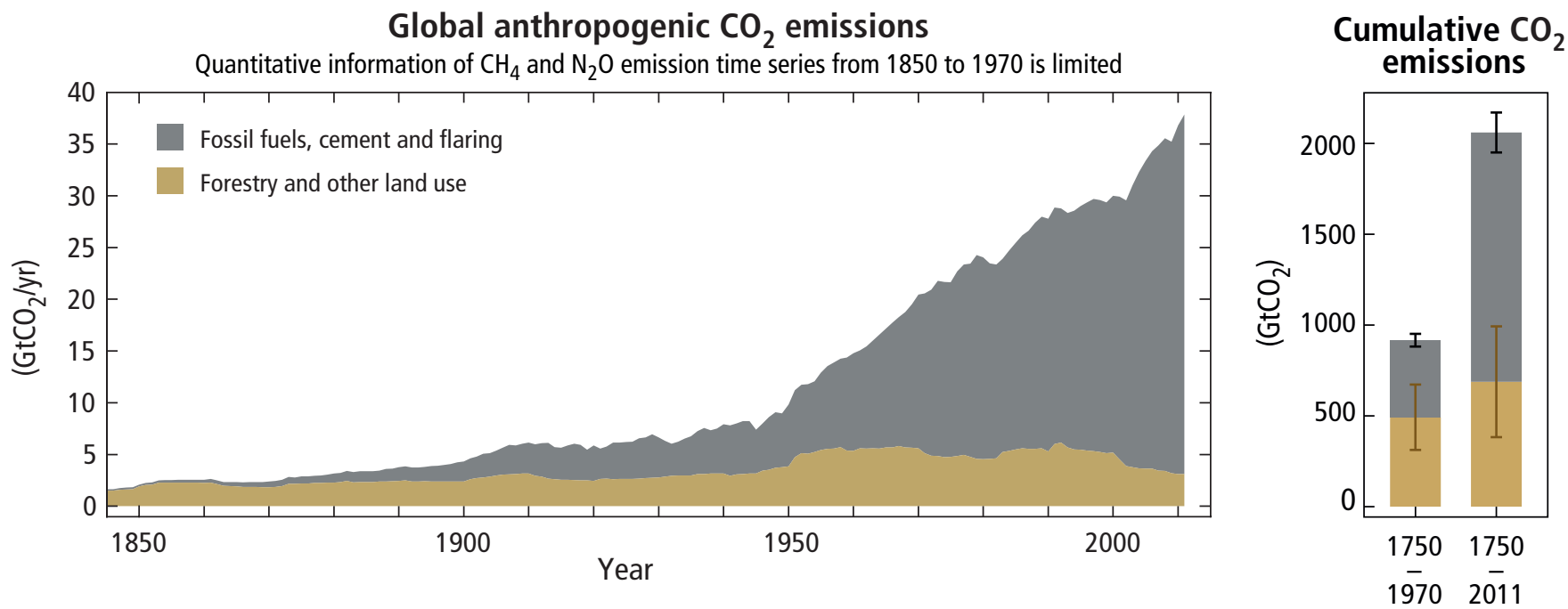

Figure 1.5 | Annual global anthropogenic carbon dioxide $\left(\mathrm{CO}_{2}\right)$ emissions (gigatonne of $\mathrm{CO}_{2}$-equivalent per year, $\mathrm{GtCO}_{2} / \mathrm{yr}$ ) from fossil fuel combustion, cement production and flaring, and forestry and other land use (FOLU), 1750-2011. Cumulative emissions and their uncertainties are shown as bars and whiskers, respectively, on the right-hand side. The global effects of the accumulation of methane $\left(\mathrm{CH}_{4}\right)$ and nitrous oxide $\left(\mathrm{N}_{2} \mathrm{O}\right)$ emissions are shown in Figure 1.3. Greenhouse gas emission data from 1970 to 2010 are shown in Figure 1.6. \{modified from WGI Figure TS.4 and WGIII Figure TS.2\}

22 Forestry and other land use (FOLU) —also referred to as LULUCF (land use, land use change and forestry)—is the subset of agriculture, forestry and other land use (AFOLU) emissions and removals of GHGs related to direct human-induced LULUCF activities, excluding agricultural emissions and removals (see WGIII AR5 Glossary).

23 Numbers from WGI 6.3 converted into $\mathrm{GtCO}_{2}$ units. Small differences in cumulative emissions from Working Group III \{WGIII SPM.3, TS.2.1\} are due to different approaches to rounding, different end years and the use of different data sets for emissions from FOLU. Estimates remain extremely close, given their uncertainties.

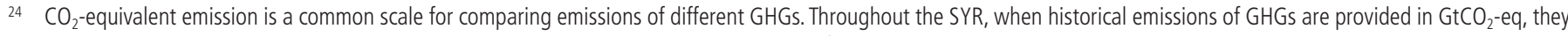
are weighted by Global Warming Potentials with a 100-year time horizon (GWP 100$)$, taken from the IPCC Second Assessment Report unless otherwise stated. A unit abbreviation of $\mathrm{GtCO}_{2}$-eq is used. \{Box 3.2, Glossary\} 
Total annual anthropogenic GHG emissions by gases 1970-2010

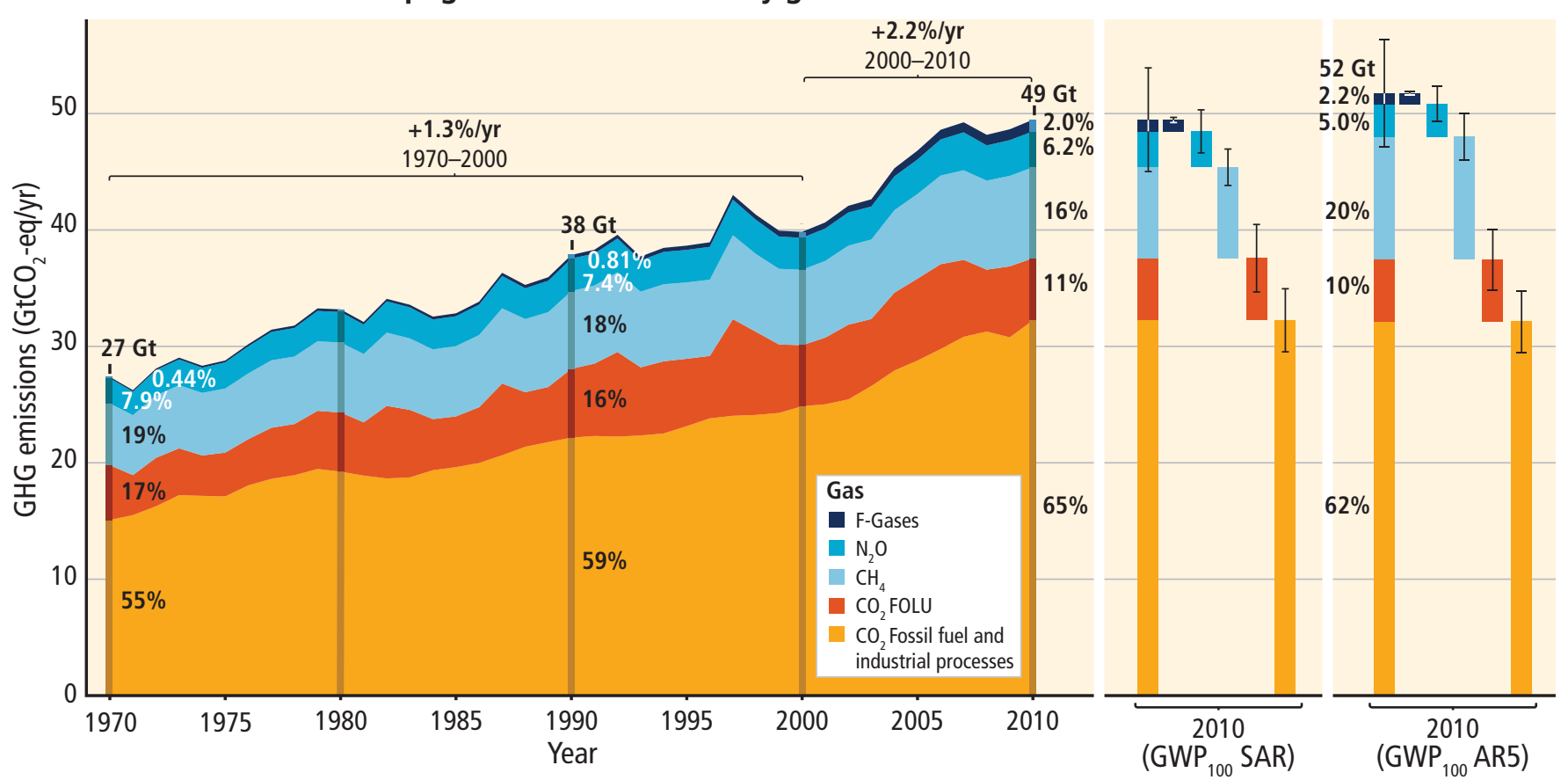

Figure 1.6 | Total annual anthropogenic greenhouse gas (GHG) emissions (gigatonne of $\mathrm{CO}_{2}$-equivalent per year, $\mathrm{GtCO}_{2}$-eq/yr) for the period 1970 to 2010 , by gases: $\mathrm{CO}_{2}$ from fossil fuel combustion and industrial processes; $\mathrm{CO}_{2}$ from Forestry and Other Land Use (FOLU); methane $\left(\mathrm{CH}_{4}\right)$; nitrous oxide $\left(\mathrm{N}_{2} \mathrm{O}\right)$; fluorinated gases covered under the Kyoto Protocol (F-gases). Right hand side shows 2010 emissions, using alternatively $\mathrm{CO}_{2}$-equivalent emission weightings based on IPCC Second Assessment Report (SAR) and AR5 values. Unless otherwise stated, $\mathrm{CO}_{2}$-equivalent emissions in this report include the basket of Kyoto gases $\left(\mathrm{CO}_{2}, \mathrm{CH}_{4}, \mathrm{~N}_{2} \mathrm{O}\right.$ as well as F-gases) calculated based on 100-year $\mathrm{Global}$ Warming Potential (GWP ${ }_{100}$ ) values from the SAR (see Glossary). Using the most recent GWP ${ }_{100}$ values from the AR5 (right-hand bars) would result in higher total annual GHG emissions $\left(52 \mathrm{GtCO}_{2}\right.$-eq/yr) from an increased contribution of methane, but does not change the long-term trend significantly. Other metric choices would change the contributions of different gases (see Box 3.2). The 2010 values are shown again broken down into their components with the associated uncertainties ( $90 \%$ confidence interval) indicated by the error bars. Global $\mathrm{CO}_{2}$ emissions from fossil fuel combustion are known with an $8 \%$ uncertainty margin ( $90 \%$ confidence interval). There are very large uncertainties (of the order of $\pm 50 \%$ ) attached to the $\mathrm{CO}_{2}$ emissions from FOLU. Uncertainty about the global emissions of $\mathrm{CH}_{4}, \mathrm{~N}_{2} \mathrm{O}$ and the F-gases has been estimated at $20 \%, 60 \%$ and $20 \%$, respectively. 2010 was the most recent year for which emission statistics on all gases as well as assessments of uncertainties were essentially complete at the time of data cut off for this report. The uncertainty estimates only account for uncertainty in emissions, not in the GWPs (as given in WGI 8.7). \{WGIII Figure SPM.1\}

$\mathrm{CO}_{2}$ emissions from fossil fuel combustion and industrial processes contributed about $78 \%$ to the total GHG emission increase between 1970 and 2010, with a contribution of similar percentage over the 2000-2010 period (high confidence). Fossil-fuel-related $\mathrm{CO}_{2}$ emissions reached $32( \pm 2.7) \mathrm{GtCO}_{2} / \mathrm{yr}$, in 2010, and grew further by about $3 \%$ between 2010 and 2011, and by about 1 to $2 \%$ between 2011 and 2012. $\mathrm{CO}_{2}$ remains the major anthropogenic $\mathrm{GHG}$, accounting for $76 \%$ of total anthropogenic GHG emissions in 2010. Of the total, $16 \%$ comes from $\mathrm{CH}_{4}, 6.2 \%$ from $\mathrm{N}_{2} \mathrm{O}$, and $2.0 \%$ from fluorinated gases (F-gases) (Figure 1.6) ${ }^{25}$. Annually, since 1970, about $25 \%$ of anthropogenic GHG emissions have been in the form of non- $\mathrm{CO}_{2}$ gases $^{26}$. \{WGIII SPM.3, 1.2, 5.2\}

Total annual anthropogenic GHG emissions have increased by about $10 \mathrm{GtCO}_{2}$-eq between 2000 and 2010. This increase directly came from the energy $(47 \%)$, industry $(30 \%)$, transport $(11 \%)$ and building (3\%) sectors (medium confidence). Accounting for indirect emissions raises the contributions by the building and industry sectors (high confidence). Since 2000, GHG emissions have been growing in all sectors, except in agriculture, forestry and other land use (AFOLU) ${ }^{22}$. In 2010, 35\% of GHG emissions were released by the energy sector, $24 \%$ (net emissions) from AFOLU, $21 \%$ by industry, $14 \%$ by transport and $6.4 \%$ by the building sector. When emissions from electricity and heat production are attributed to the sectors that use the final energy (i.e., indirect emissions), the shares of the industry and building sectors in global GHG emissions are increased to $31 \%$ and $19 \%$, respectively (Figure 1.7). \{WGIII SPM.3, 7.3, 8.1, 9.2, 10.3, 11.2\} See also Box 3.2 for contributions from various sectors, based on metrics other than 100-year Global Warming Potential $\left(\mathrm{GWP}_{100}\right)$.

Globally, economic and population growth continue to be the most important drivers of increases in $\mathrm{CO}_{2}$ emissions from fossil fuel combustion. The contribution of population growth between 2000 and 2010 remained roughly identical to that of the previous three decades, while the contribution of economic growth has risen sharply (high confidence). Between 2000 and

25 Using the most recent 100-year Global Warming Potential $\left(\mathrm{GWP}_{100}\right)$ values from the AR5 \{WGI 8.7$\}$ instead of GWP 100 $_{10}$ values from the IPCC Second Assessment Report, global GHG emission totals would be slightly higher ( $52 \mathrm{GtCO}_{2}$-eq/yr) and non- $\mathrm{CO}_{2}$ emission shares would be $20 \%$ for $\mathrm{CH}_{4}, 5 \%$ for $\mathrm{N}_{2} \mathrm{O}$ and $2.2 \%$ for $\mathrm{F}$-gases.

26 For this report, data on non- $\mathrm{CO}_{2}$ GHGs, including F-gases, were taken from the Electronic Data Gathering, Analysis, and Retrieval (EDGAR) database \{WGIII Annex II.9\}, which covers substances included in the Kyoto Protocol in its first commitment period. 


\section{Greenhouse gas emissions by economic sectors}

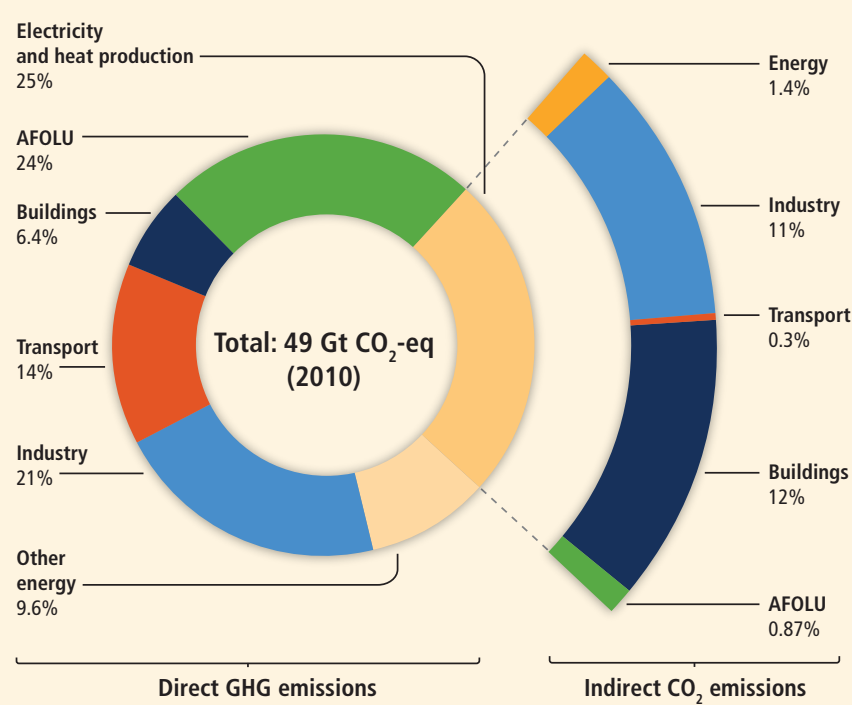

Figure 1.7 | Total anthropogenic greenhouse gas (GHG) emissions (gigatonne of $\mathrm{CO}_{2}$ equivalent per year, $\mathrm{GtCO}_{2}$-eq/yr) from economic sectors in 2010. The circle shows the shares of direct GHG emissions (in \% of total anthropogenic GHG emissions) from five economic sectors in 2010. The pull-out shows how shares of indirect $\mathrm{CO}_{2}$ emissions (in \% of total anthropogenic GHG emissions) from electricity and heat production are attributed to sectors of final energy use. 'Other energy' refers to all GHG emission sources in the energy sector as defined in WGIII Annex II, other than electricity and heat production \{WGIII Annex II.9.1\}. The emission data on agriculture, forestry and other land use (AFOLU) includes land-based $\mathrm{CO}_{2}$ emissions from forest fires, peat fires and peat decay that approximate to net $\mathrm{CO}_{2}$ flux from the sub-sectors of forestry and other land use (FOLU) as described in Chapter 11 of the WGIII report. Emissions are converted into $\mathrm{CO}_{2}$-equivalents based on 100-year Global Warming Potential ( $\left.\mathrm{GWP}_{100}\right)$, taken from the IPCC Second Assessment Report (SAR). Sector definitions are provided in WGIII Annex II.9. \{WGIII Figure SPM.2\}
2010, both drivers outpaced emission reductions from improvements in energy intensity of gross domestic product (GDP) (Figure 1.8). Increased use of coal relative to other energy sources has reversed the long-standing trend in gradual decarbonization (i.e., reducing the carbon intensity of energy) of the world's energy supply. \{WGIII SPM.3, $T S .2 .2,1.3,5.3,7.2,7.3,14.3\}$

\subsection{Attribution of climate changes and impacts}

The evidence for human influence on the climate system has grown since AR4. Human influence has been detected in warming of the atmosphere and the ocean, in changes in the global water cycle, in reductions in snow and ice, and in global mean sea level rise; and it is extremely likely to have been the dominant cause of the observed warming since the mid20th century. In recent decades, changes in climate have caused impacts on natural and human systems on all continents and across the oceans. Impacts are due to observed climate change, irrespective of its cause, indicating the sensitivity of natural and human systems to changing climate.

The causes of observed changes in the climate system, as well as in any natural or human system impacted by climate, are established following a consistent set of methods. Detection addresses the question of whether climate or a natural or human system affected by climate has actually changed in a statistical sense, while attribution evaluates the relative contributions of multiple causal factors to an observed change

Decomposition of the change in total global $\mathrm{CO}_{2}$ emissions from fossil fuel combustion by decade

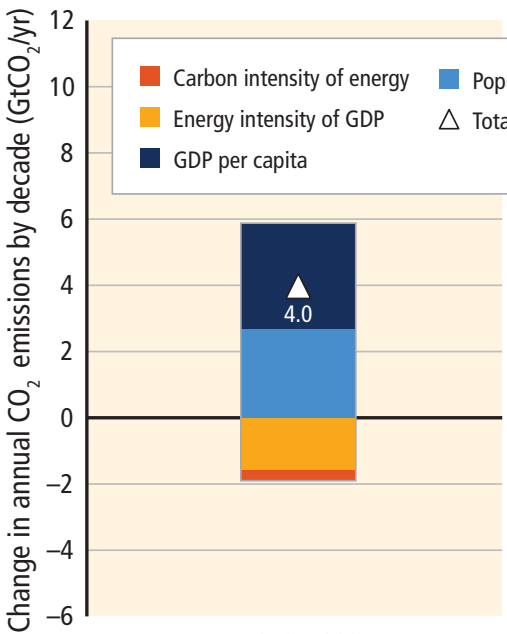

1970-1980
Population

Total change

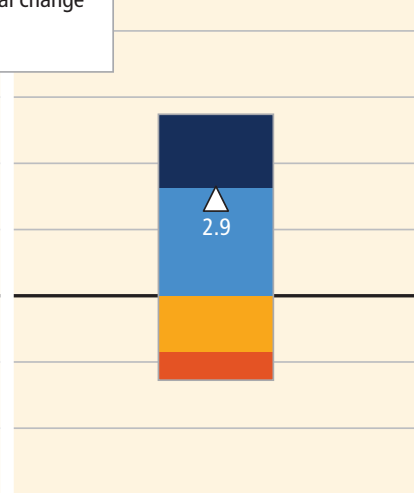

1980-1990

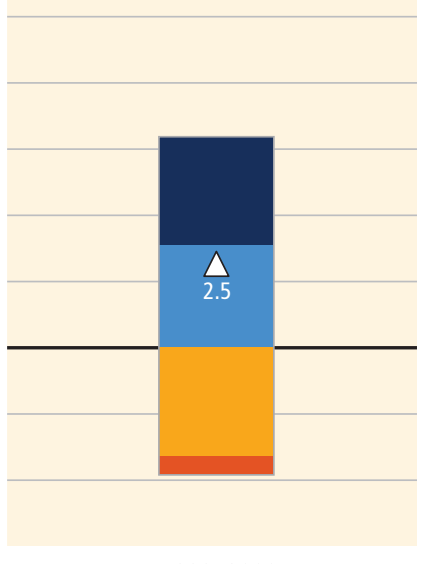

1990-2000

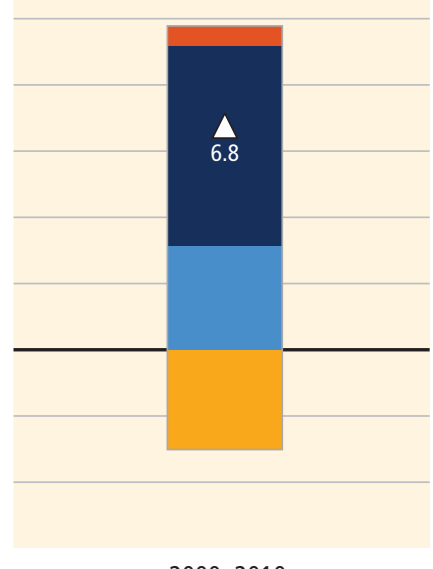

2000-2010

Figure 1.8 Decomposition of the change in total annual carbon dioxide $\left(\mathrm{CO}_{2}\right)$ emissions from fossil fuel combustion by decade and four driving factors: population, income (gross domestic product, GDP) per capita, energy intensity of GDP and carbon intensity of energy. The bar segments show the changes associated with each individual factor, holding the respective other factors constant. Total emission changes are indicated by a triangle. The change in emissions over each decade is measured in gigatonnes of $\mathrm{CO}_{2}$ per year $\left(\mathrm{GtCO}_{2} / \mathrm{yr}\right)$; income is converted into common units, using purchasing power parities. \{WGIII SPM.3\} 
or event with an assignment of statistical confidence ${ }^{27}$. Attribution of climate change to causes quantifies the links between observed climate change and human activity, as well as other, natural, climate drivers. In contrast, attribution of observed impacts to climate change considers the links between observed changes in natural or human systems and observed climate change, regardless of its cause. Results from studies attributing climate change to causes provide estimates of the magnitude of warming in response to changes in radiative forcing and hence support projections of future climate change (Topic 2). Results from studies attributing impacts to climate change provide strong indications for the sensitivity of natural or human systems to future climate change. \{WGI 10.8, WGII SPM A-1, WGI/IIIII/SYR Glossaries\}

\subsubsection{Attribution of climate changes to human and} natural influences on the climate system

It is extremely likely that more than half of the observed increase in global average surface temperature from 1951 to 2010 was caused by the anthropogenic increase in GHG concentrations and other anthropogenic forcings together (Figure 1.9). The best estimate of the human induced contribution to warming is similar to the observed warming over this period. GHGs contributed a global mean surface warming likely to be in the range of $0.5^{\circ} \mathrm{C}$ to $1.3^{\circ} \mathrm{C}$ over the period 1951 to 2010, with further contributions from other anthropogenic forcings, including the cooling effect of aerosols, from natural forcings, and from natural internal variability (see Figure 1.9).

Contributions to observed surface temperature change over the period 1951-2010

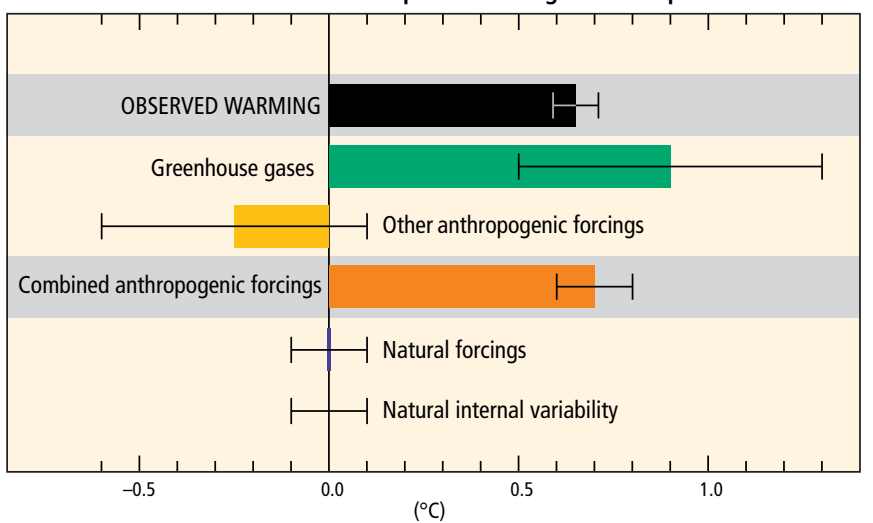

Figure 1.9 | Assessed likely ranges (whiskers) and their mid-points (bars) for warming trends over the 1951-2010 period from well-mixed greenhouse gases, other anthropogenic forcings (including the cooling effect of aerosols and the effect of land use change), combined anthropogenic forcings, natural forcings, and natural internal climate variability (which is the element of climate variability that arises spontaneously within the climate system, even in the absence of forcings). The observed surface temperature change is shown in black, with the 5 to $95 \%$ uncertainty range due to observational uncertainty. The attributed warming ranges (colours) are based on observations combined with climate model simulations, in order to estimate the contribution by an individual external forcing to the observed warming. The contribution from the combined anthropogenic forcings can be estimated with less uncertainty than the separate contributions from greenhouse gases and other anthropogenic forcings separately. This is because these two contributions partially compensate, resulting in a signal that is better constrained by observations. \{Based on Figure WGI TS. 10\}
Together these assessed contributions are consistent with the observed warming of approximately $0.6^{\circ} \mathrm{C}$ to $0.7^{\circ} \mathrm{C}$ over this period. \{WGI SPM D.3, 10.3.1\}

It is very likely that anthropogenic influence, particularly GHGs and stratospheric ozone depletion, has led to a detectable observed pattern of tropospheric warming and a corresponding cooling in the lower stratosphere since 1961. \{WGI SPM D.3, 2.4.4, 9.4.1, 10.3.1\}

Over every continental region except Antarctica, anthropogenic forcings have likely made a substantial contribution to surface temperature increases since the mid-20th century (Figure 1.10). For Antarctica, large observational uncertainties result in low confidence that anthropogenic forcings have contributed to the observed warming averaged over available stations. In contrast, it is likely that there has been an anthropogenic contribution to the very substantial Arctic warming since the mid-20th century. Human influence has likely contributed to temperature increases in many sub-continental regions. $\{W G I$ SPM D.3, TS.4.8, 10.3.1\}

Anthropogenic influences have very likely contributed to Arctic sea ice loss since 1979 (Figure 1.10). There is low confidence in the scientific understanding of the small observed increase in Antarctic sea ice extent due to the incomplete and competing scientific explanations for the causes of change and low confidence in estimates of natural internal variability in that region. $\{W G I S P M D .3,10.5 .1$, Figure 10.16\}

Anthropogenic influences likely contributed to the retreat of glaciers since the 1960s and to the increased surface melting of the Greenland ice sheet since 1993. Due to a low level of scientific understanding, however, there is low confidence in attributing the causes of the observed loss of mass from the Antarctic ice sheet over the past two decades. It is likely that there has been an anthropogenic contribution to observed reductions in Northern Hemisphere spring snow cover since 1970. \{WGI 4.3.3, 10.5.2, 10.5.3\}

It is likely that anthropogenic influences have affected the global water cycle since 1960. Anthropogenic influences have contributed to observed increases in atmospheric moisture content (medium confidence), to global-scale changes in precipitation patterns over land (medium confidence), to intensification of heavy precipitation over land regions where data are sufficient (medium confidence) (see 1.4) and to changes in surface and subsurface ocean salinity (very likely). \{WGI SPM D.3, 2.5.1, 2.6.2, 3.3.2, 3.3.3, 7.6.2, 10.3.2, 10.4.2, 10.6\}

It is very likely that anthropogenic forcings have made a substantial contribution to increases in global upper ocean heat content $(0-700 \mathrm{~m})$ observed since the 1970s (Figure 1.10). There is evidence for human influence in some individual ocean basins. It is very likely that there is a substantial anthropogenic contribution to the global mean sea level rise since the 1970s. This is based on the high confidence in an anthropogenic influence on the two largest contributions to sea level rise: thermal expansion and glacier mass loss. Oceanic 

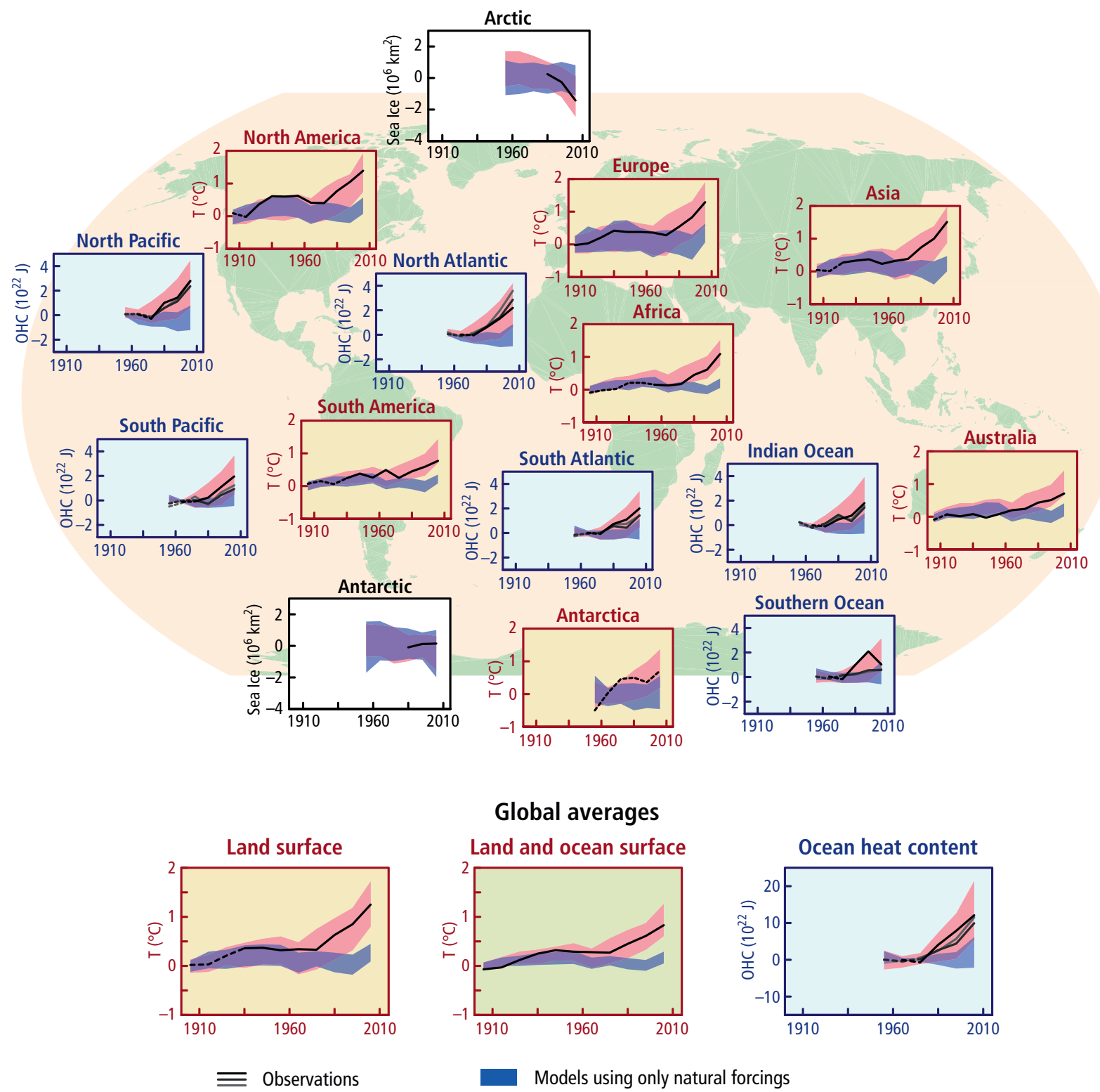

Global averages
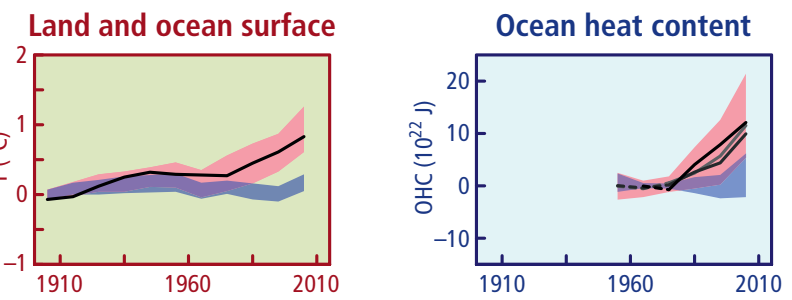

Models using only natural forcings

Models using both natural and anthropogenic forcings

Figure 1.10 Comparison of observed and simulated change in continental surface temperatures on land (yellow panels), Arctic and Antarctic September sea ice extent (white panels), and upper ocean heat content in the major ocean basins (blue panels). Global average changes are also given. Anomalies are given relative to 1880-1919 for surface temperatures, to 1960-1980 for ocean heat content, and to 1979-1999 for sea ice. All time series are decadal averages, plotted at the centre of the decade. For temperature panels, observations are dashed lines if the spatial coverage of areas being examined is below $50 \%$. For ocean heat content and sea ice panels, the solid lines are where the coverage of data is good and higher in quality, and the dashed lines are where the data coverage is only adequate, and, thus, uncertainty is larger (note that different lines indicate different data sets; for details, see WGI Figure SPM.6). Model results shown are Coupled Model Intercomparison Project Phase 5 (CMIP5) multi-model ensemble ranges, with shaded bands indicating the 5 to 95\% confidence intervals. \{WGI Figure SPM 6; for detail, see WGI Figure TS.12\}

uptake of anthropogenic $\mathrm{CO}_{2}$ has resulted in gradual acidification of ocean surface waters (high confidence). \{WGI SPM D.3, 3.2.3, 3.8.2, 10.4.1, 10.4.3, 10.4.4, 10.5.2, 13.3, Box 3.2, TS.4.4, WGII 6.1.1.2, Box $C C-O A\}$

\subsubsection{Observed impacts attributed to climate change}

In recent decades, changes in climate have caused impacts on natural and human systems on all continents and across the oceans. Impacts are due to observed climate change, irrespective of its cause, indicating the sensitivity of natural and human systems to changing climate. Evidence of observed climate change impacts is strongest and most comprehensive for natural systems. Some impacts on human systems have also been attributed to climate change, with a major or minor contribution of climate change distinguishable from other influences (Figure 1.11). Impacts on human systems are often geographically heterogeneous because they depend not only on changes in climate variables but also on social and economic factors. Hence, the changes are more easily observed at local levels, while attribution can remain difficult. \{WGII SPM A-1, SPM A-3, 18.1, 18.3-18.6\} 


\section{(a) Widespread impacts attributed to climate change based on the available scientific literature since the AR4}

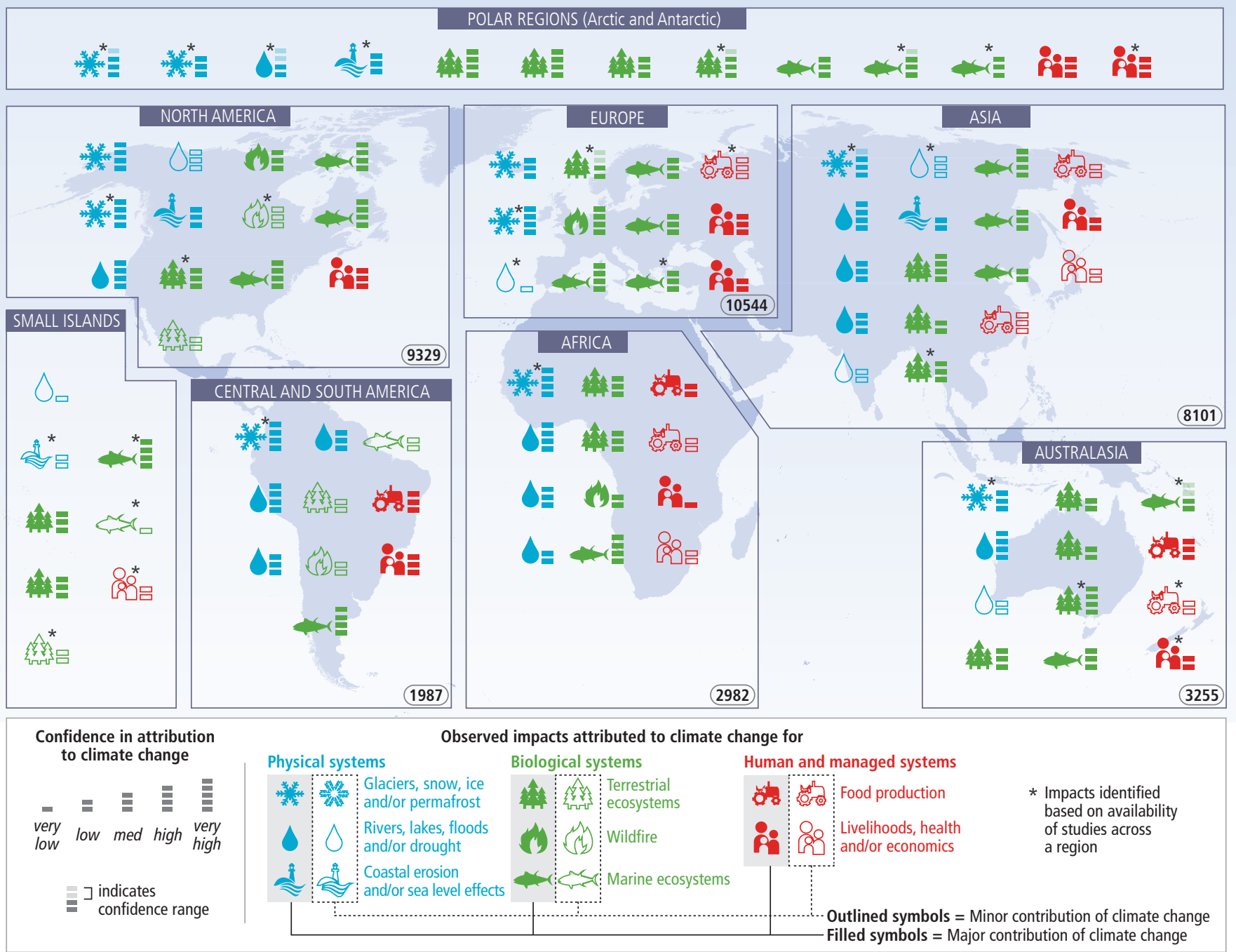

(b)

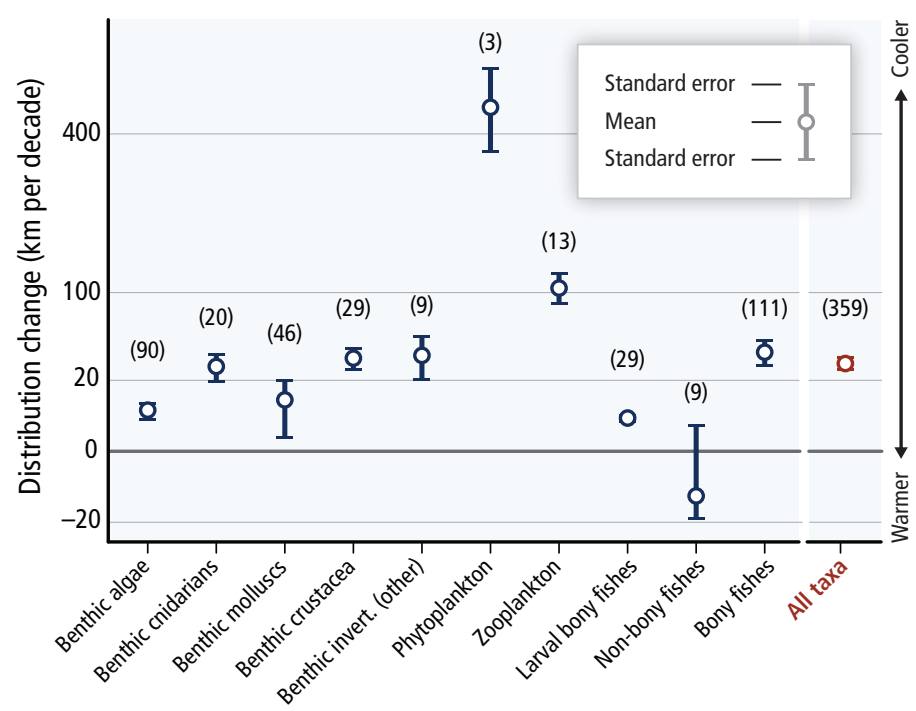

(c)

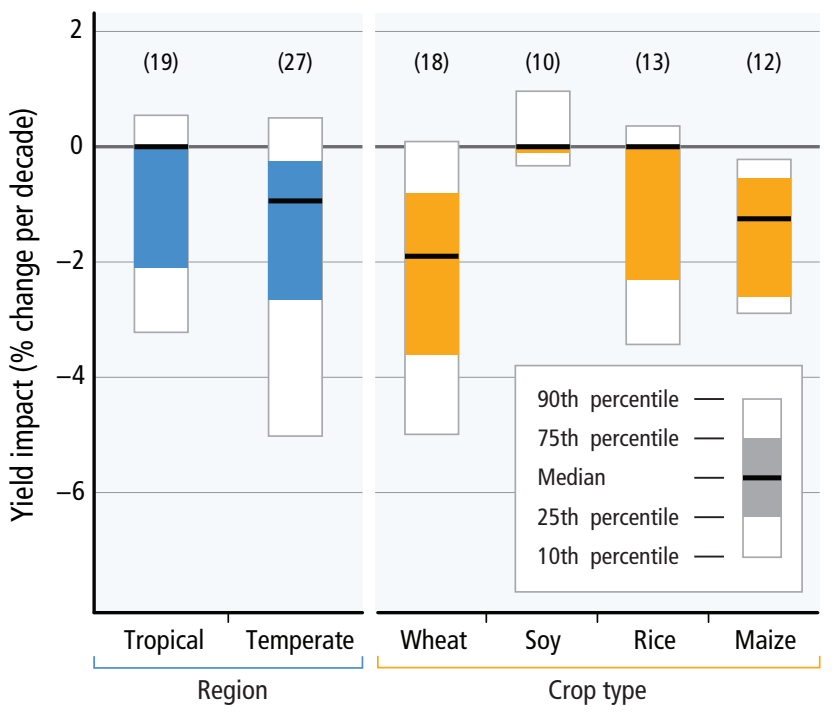


Figure 1.11 | Widespread impacts in a changing world: (a) Based on the available scientific literature since the IPCC Fourth Assessment Report (AR4), there are substantially more impacts in recent decades now attributed to climate change. Attribution requires defined scientific evidence on the role of climate change. Absence from the map of additional impacts attributed to climate change does not imply that such impacts have not occurred. The publications supporting attributed impacts reflect a growing knowledge base, but publications are still limited for many regions, systems and processes, highlighting gaps in data and studies. Symbols indicate categories of attributed impacts, the relative contribution of climate change (major or minor) to the observed impact and confidence in attribution. Each symbol refers to one or more entries in WGII Table SPM.A1, grouping related regional-scale impacts. Numbers in ovals indicate regional totals of climate change publications from 2001 to 2010, based on the Scopus bibliographic database for publications in English with individual countries mentioned in title, abstract or key words (as of July 2011). These numbers provide an overall measure of the available scientific literature on climate change across regions; they do not indicate the number of publications supporting attribution of climate change impacts in each region. Studies for polar regions and small islands are grouped with neighbouring continental regions. The inclusion of publications for assessment of attribution followed IPCC scientific evidence criteria defined in WGII Chapter 18. Publications considered in the attribution analyses come from a broader range of literature assessed in the WGII AR5. See WGII Table SPM.A1 for descriptions of the attributed impacts. (b) Average rates of change in distribution ( $\mathrm{km}$ per decade) for marine taxonomic groups based on observations over 1900-2010. Positive distribution changes are consistent with warming (moving into previously cooler waters, generally poleward). The number of responses analysed is given for each category. (c) Summary of estimated impacts of observed climate changes on yields over 1960-2013 for four major crops in temperate and tropical regions, with the number of data points analysed given within parentheses for each category. \{WGIl Figure SPM.2, Box TS. 1 Figure 1\}

In many regions, changing precipitation or melting snow and ice are altering hydrological systems, affecting water resources in terms of quantity and quality (medium confidence). Glaciers continue to shrink almost worldwide due to climate change (high confidence), affecting runoff and water resources downstream (medium confidence). Climate change is causing permafrost warming and thawing in high-latitude regions and in high-elevation regions (high confidence). $\{$ WGII SPM A-1\}

Many terrestrial, freshwater and marine species have shifted their geographic ranges, seasonal activities, migration patterns, abundances and species interactions in response to ongoing climate change (high confidence). While only a few recent species extinctions have been attributed as yet to climate change (high confidence), natural global climate change at rates slower than current anthropogenic climate change caused significant ecosystem shifts and species extinctions during the past millions of years (high confidence). Increased tree mortality, observed in many places worldwide, has been attributed to climate change in some regions. Increases in the frequency or intensity of ecosystem disturbances such as droughts, windstorms, fires and pest outbreaks have been detected in many parts of the world and in some cases are attributed to climate change (medium confidence). Numerous observations over the last decades in all ocean basins show changes in abundance, distribution shifts poleward and/ or to deeper, cooler waters for marine fishes, invertebrates and phytoplankton (very high confidence), and altered ecosystem composition (high confidence), tracking climate trends. Some warm-water corals and their reefs have responded to warming with species replacement, bleaching, and decreased coral cover causing habitat loss (high confidence). Some impacts of ocean acidification on marine organisms have been attributed to human influence, from the thinning of pteropod and foraminiferan shells (medium confidence) to the declining growth rates of corals (low confidence). Oxygen minimum zones are progressively expanding in the tropical Pacific, Atlantic and Indian Oceans, due to reduced ventilation and $\mathrm{O}_{2}$ solubility in warmer, more stratified oceans, and are constraining fish habitat (medium confidence). \{WGII SPMA-1, Table SPM.A1, TS A-1, 6.3.2.5, 6.3.3, 18.3-18.4, 30.5.1.1, Box CC-OA, Box CC-CR\}
Assessment of many studies covering a wide range of regions and crops shows that negative impacts of climate change on crop yields have been more common than positive impacts (high confidence). The smaller number of studies showing positive impacts relate mainly to high-latitude regions, though it is not yet clear whether the balance of impacts has been negative or positive in these regions (high confidence). Climate change has negatively affected wheat and maize yields for many regions and in the global aggregate (medium confidence). Effects on rice and soybean yield have been smaller in major production regions and globally, with a median change of zero across all available data which are fewer for soy compared to the other crops (see Figure 1.11c). Observed impacts relate mainly to production aspects of food security rather than access or other components of food security. Since AR4, several periods of rapid food and cereal price increases following climate extremes in key producing regions indicate a sensitivity of current markets to climate extremes among other factors (medium confidence). $\{W G I / S P M A-1\}$

At present the worldwide burden of human ill-health from climate change is relatively small compared with effects of other stressors and is not well quantified. However, there has been increased heat-related mortality and decreased cold-related mortality in some regions as a result of warming (medium confidence). Local changes in temperature and rainfall have altered the distribution of some water-borne illnesses and disease vectors (medium confidence). $\{W G I I S P M A-1\}$

'Cascading' impacts of climate change can now be attributed along chains of evidence from physical climate through to intermediate systems and then to people (Figure 1.12). The changes in climate feeding into the cascade, in some cases, are linked to human drivers (e.g., a decreasing amount of water in spring snowpack in western North America), while, in other cases, assessments of the causes of observed climate change leading into the cascade are not available. In all cases, confidence in detection and attribution to observed climate change decreases for effects further down each impact chain. \{WGII 18.6.3\} 


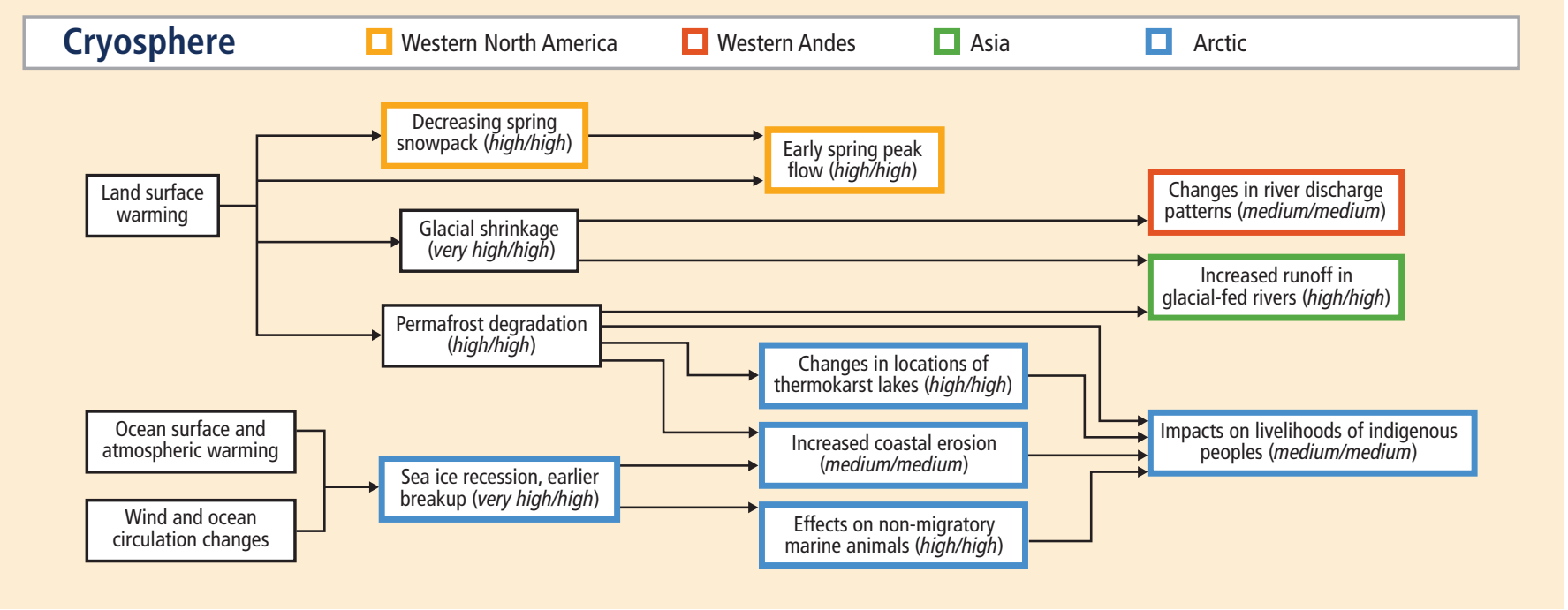

\begin{tabular}{|l|l|l|}
\hline Ocean $\quad \square$ Physical impacts $\quad \square$ Biological impacts $\quad \square$ Impacts on managed systems \\
\hline
\end{tabular}
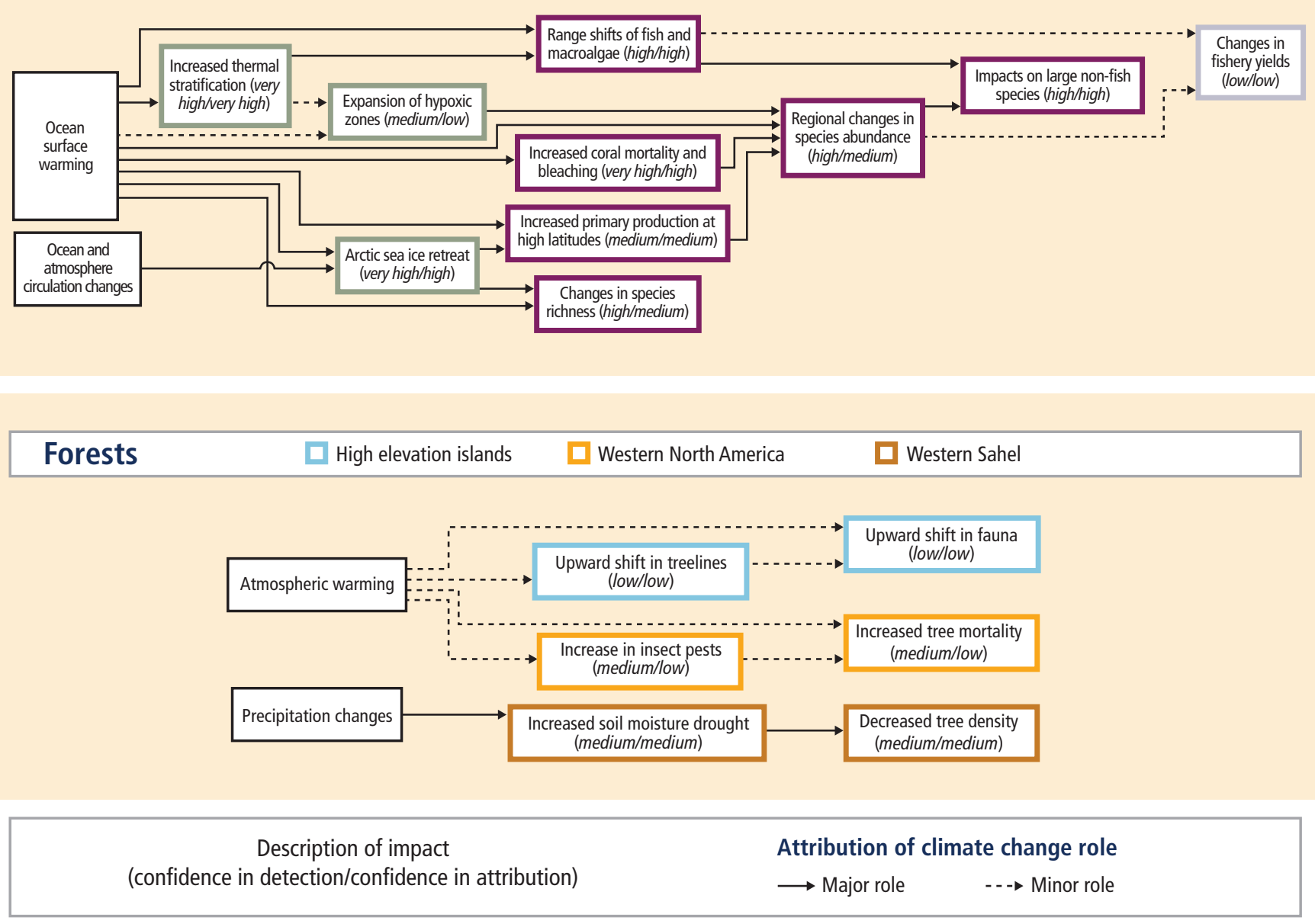

Figure 1.12 | Major systems where new evidence indicates interconnected, 'cascading' impacts from recent climate change through several natural and human subsystems. Bracketed text indicates confidence in the detection of a climate change effect and the attribution of observed impacts to climate change. The role of climate change can be major (solid arrow) or minor (dashed arrow). Initial evidence indicates that ocean acidification is following similar trends with respect to impact on human systems as ocean warming. \{WGII Figure 18-4\} 


\section{$1.4 \quad$ Extreme events}

Changes in many extreme weather and climate events have been observed since about 1950 . Some of these changes have been linked to human influences, including a decrease in cold temperature extremes, an increase in warm temperature extremes, an increase in extreme high sea levels and an increase in the number of heavy precipitation events in a number of regions.

It is very likely that the number of cold days and nights has decreased and the number of warm days and nights has increased on the global scale. It is likely that the frequency of heat waves has increased in large parts of Europe, Asia and Australia. It is very likely that human influence has contributed to the observed global scale changes in the frequency and intensity of daily temperature extremes since the mid-20th century. It is likely that human influence has more than doubled the probability of occurrence of heat waves in some locations. \{WGI SPM B.1, SPM D.3, Table SPM.1, FAQ 2.2, 2.6.1, $10.6\}$

There is medium confidence that the observed warming has increased heat-related human mortality and decreased coldrelated human mortality in some regions. Extreme heat events currently result in increases in mortality and morbidity in North America (very high confidence), and in Europe with impacts that vary according to people's age, location and socio-economic factors (high confidence). \{WGII SPM A-1, 11.4.1, Table 23-1, 26.6.1.2\}

There are likely more land regions where the number of heavy precipitation events has increased than where it has decreased. The frequency and intensity of heavy precipitation events has likely increased in North America and Europe. In other continents, confidence in trends is at most medium. It is very likely that global near-surface and tropospheric air specific humidity has increased since the 1970s. In land regions where observational coverage is sufficient for assessment, there is medium confidence that anthropogenic forcing has contributed to a global-scale intensification of heavy precipitation over the second half of the 20th century. \{WGI SPM B-1, 2.5.1, 2.5.4-2.5.5, 2.6.2, 10.6, Table SPM.1, FAQ 2.2, SREX Table 3-1, 3.2\}

There is low confidence that anthropogenic climate change has affected the frequency and magnitude of fluvial floods on a global scale. The strength of the evidence is limited mainly by a lack of long-term records from unmanaged catchments. Moreover, floods are strongly influenced by many human activities impacting catchments, making the attribution of detected changes to climate change difficult. However, recent detection of increasing trends in extreme precipitation and discharges in some catchments implies greater risks of flooding on a regional scale (medium confidence). Costs related to flood damage, worldwide, have been increasing since the 1970s, although this is partly due to the increasing exposure of people and assets. \{WGI 2.6.2, WGII 3.2.7, SREX SPM B\}
There is low confidence in observed global-scale trends in droughts, due to lack of direct observations, dependencies of inferred trends on the choice of the definition for drought, and due to geographical inconsistencies in drought trends. There is also low confidence in the attribution of changes in drought over global land areas since the mid-20th century, due to the same observational uncertainties and difficulties in distinguishing decadal scale variability in drought from long-term trends. \{WGI Table SPM.1, 2.6.2.3, 10.6, Figure 2.33, WGII 3.ES, 3.2.7\}

There is low confidence that long-term changes in tropical cyclone activity are robust, and there is low confidence in the attribution of global changes to any particular cause. However, it is virtually certain that intense tropical cyclone activity has increased in the North Atlantic since 1970. \{WGI Table SPM.1, 2.6.3, 10.6\}

It is likely that extreme sea levels (for example, as experienced in storm surges) have increased since 1970, being mainly the result of mean sea level rise. Due to a shortage of studies and the difficulty of distinguishing any such impacts from other modifications to coastal systems, limited evidence is available on the impacts of sea level rise. \{WGI 3.7.4-3.7.6, Figure 3.15, WGII 5.3.3.2, 18.3\}

Impacts from recent climate-related extremes, such as heat waves, droughts, floods, cyclones and wildfires, reveal significant vulnerability and exposure of some ecosystems and many human systems to current climate variability (very high confidence). Impacts of such climate-related extremes include alteration of ecosystems, disruption of food production and water supply, damage to infrastructure and settlements, human morbidity and mortality and consequences for mental health and human well-being. For countries at all levels of development, these impacts are consistent with a significant lack of preparedness for current climate variability in some sectors. \{WGII SPM A-1, 3.2, 4.2-3, 8.1, 9.3, 10.7, 11.3, 11.7, 13.2, 14.1, 18.6, 22.2.3, 22.3, 23.3.1.2, 24.4.1, 25.6-8, 26.6-7, 30.5, Table 18-3, Table 23-1, Figure 26-2, Box 4-3, Box 4-4, Box 25-5, Box 25-6, Box 25-8, Box CC-CR\}

Direct and insured losses from weather-related disasters have increased substantially in recent decades, both globally and regionally. Increasing exposure of people and economic assets has been the major cause of long-term increases in economic losses from weather- and climate-related disasters (high confidence). \{WGII 10.7.3, SREX SPM B, 4.5.3.3\} 


\subsection{Exposure and vulnerability}

The character and severity of impacts from climate change and extreme events emerge from risk that depends not only on climate-related hazards but also on exposure (people and assets at risk) and vulnerability (susceptibility to harm) of human and natural systems.

Exposure and vulnerability are influenced by a wide range of social, economic and cultural factors and processes that have been incompletely considered to date and that make quantitative assessments of their future trends difficult (high confidence). These factors include wealth and its distribution across society, demographics, migration, access to technology and information, employment patterns, the quality of adaptive responses, societal values, governance structures and institutions to resolve conflict. \{WGII SPM A-3, SREX SPM B\}

Differences in vulnerability and exposure arise from non-climatic factors and from multidimensional inequalities often produced by uneven development processes (very high confidence). These differences shape differential risks from climate change. People who are socially, economically, culturally, politically, institutionally or otherwise marginalized are especially vulnerable to climate change and also to some adaptation and mitigation responses (medium evidence, high agreement). This heightened vulnerability is rarely due to a single cause. Rather, it is the product of intersecting social processes that result in inequalities in socio-economic status and income, as well as in exposure. Such social processes include, for example, discrimination on the basis of gender, class, ethnicity, age and (dis)ability. \{WGII SPM A-1, Figure SPM.1, 8.1-8.2, 9.3-9.4, 10.9, 11.1, 11.3-11.5, 12.2-12.5, 13.1-13.3, 14.1-14.3, 18.4, 19.6, 23.5, $25.8,26.6,26.8,28.4$, Box CC-GC\}

Climate-related hazards exacerbate other stressors, often with negative outcomes for livelihoods, especially for people living in poverty (high confidence). Climate-related hazards affect poor people's lives directly through impacts on livelihoods, reductions in crop yields or the destruction of homes, and indirectly through, for example, increased food prices and food insecurity. Observed positive effects for poor and marginalized people, which are limited and often indirect, include examples such as diversification of social networks and of agricultural practices. \{WGII SPM A-1, 8.2-8.3, 9.3, 11.3, 13.1$13.3,22.3,24.4,26.8\}$

Violent conflict increases vulnerability to climate change (medium evidence, high agreement). Large-scale violent conflict harms assets that facilitate adaptation, including infrastructure, institutions, natural resources, social capital and livelihood opportunities. \{WGII SPM A-1, 12.5, 19.2, 19.6\}

\subsection{Human responses to climate change: adaptation and mitigation}

Adaptation and mitigation experience is accumulating across regions and scales, even while global anthropogenic greenhouse gas emissions have continued to increase.

Throughout history, people and societies have adjusted to and coped with climate, climate variability and extremes, with varying degrees of success. In today's changing climate, accumulating experience with adaptation and mitigation efforts can provide opportunities for learning and refinement $(3,4)$. \{WGII SPM A-2\}

Adaptation is becoming embedded in some planning processes, with more limited implementation of responses (high confidence). Engineered and technological options are commonly implemented adaptive responses, often integrated within existing programmes, such as disaster risk management and water management. There is increasing recognition of the value of social, institutional and ecosystem-based measures and of the extent of constraints to adaptation. \{WGII SPM A-2, 4.4, 5.5, 6.4, 8.3, 9.4, 11.7, 14.1, 14.3-14.4, 15.2-15.5, 17.2-17.3, 21.3, 21.5, 22.4, 23.7, 25.4, 26.8-26.9, 30.6, Box 25-1, Box 25-2, Box 25-9, Box CC-EA\}

Governments at various levels have begun to develop adaptation plans and policies and integrate climate change considerations into broader development plans. Examples of adaptation are now available from all regions of the world (see Topic 4 for details on adaptation options and policies to support their implementation). \{WGII SPM A-2, 22.4, 23.7, 24.4-24.6, 24.9, 25.4, 25.10, 26.7-26.9, 27.3, 28.2, 28.4, 29.3, 29.6, 30.6, Table 25-2, Table 29-3, Figure 29-1, Box 5-1, Box 23-3, Box 25-1, Box 25-2, Box 25-9, Box CC-TC\}

Global increases in anthropogenic emissions and climate impacts have occurred, even while mitigation activities have taken place in many parts of the world. Though various mitigation initiatives between the sub-national and global scales have been developed or implemented, a full assessment of their impact may be premature. $\{$ WGIII SPM.3, SPM.5\} 


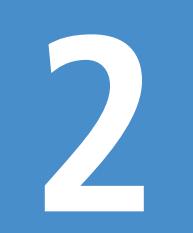

\section{Future Climate Changes, Risks and Impacts}




\section{Topic 2: Future Climate Changes, Risk and Impacts}

Continued emission of greenhouse gases will cause further warming and long-lasting changes in all components of the climate system, increasing the likelihood of severe, pervasive and irreversible impacts for people and ecosystems. Limiting climate change would require substantial and sustained reductions in greenhouse gas emissions which, together with adaptation, can limit climate change risks.

Topic 2 assesses projections of future climate change and the resulting risks and impacts. Factors that determine future climate change, including scenarios for future greenhouse gas (GHG) emissions, are outlined in Section 2.1. Descriptions of the methods and tools used to make projections of climate, impacts and risks, and their development since the IPCC Fourth Assessment Report (AR4), are provided in Boxes 2.1 to 2.3. Details of projected changes in the climate system, including the associated uncertainty and the degree of expert confidence in the projections are provided in Section 2.2. The future impacts of climate change on natural and human systems and associated risks are assessed in Section 2.3. Topic 2 concludes with an assessment of irreversible changes, abrupt changes and changes beyond 2100 in Section 2.4.

\subsection{Key drivers of future climate and the basis on which projections are made}

Cumulative emissions of $\mathrm{CO}_{2}$ largely determine global mean surface warming by the late 21 st century and beyond. Projections of greenhouse gas emissions vary over a wide range, depending on both socio-economic development and climate policy.

Climate models are mathematical representations of processes important in the Earth's climate system. Results from a hierarchy of climate models are considered in this report; ranging from simple idealized models, to models of intermediate complexity, to comprehensive General Circulation Models (GCMs), including Earth System Models (ESMs) that also simulate the carbon cycle. The GCMs simulate many climate aspects, including the temperature of the atmosphere and the oceans, precipitation, winds, clouds, ocean currents and sea-ice extent. The models are extensively tested against historical observations (Box 2.1). $\{W G \mid$ 1.5.2, 9.1.2, 9.2, 9.8.1\}

In order to obtain climate change projections, the climate models use information described in scenarios of GHG and air pollutant emissions and land use patterns. Scenarios are generated by a range of approaches, from simple idealised experiments to Integrated Assessment Models (IAMs, see Glossary). Key factors driving changes in anthropogenic GHG emissions are economic and population growth, lifestyle and behavioural changes, associated changes in energy use and land use, technology and climate policy, which are fundamentally uncertain. $\{$ WGI 11.3, 12.4, WGIII 5, 6, 6.1\}

The standard set of scenarios used in the AR5 is called Representative Concentration Pathways (RCPs, Box 2.2). \{WGI Box SPM.1\}

\section{Box 2.1 | Advances, Confidence and Uncertainty in Modelling the Earth's Climate System}

Improvements in climate models since the IPCC Fourth Assessment Report (AR4) are evident in simulations of continentalscale surface temperature, large-scale precipitation, the monsoon, Arctic sea ice, ocean heat content, some extreme events, the carbon cycle, atmospheric chemistry and aerosols, the effects of stratospheric ozone and the El Niño-Southern Oscillation. Climate models reproduce the observed continental-scale surface temperature patterns and multi-decadal trends, including the more rapid warming since the mid-20th century and the cooling immediately following large volcanic eruptions (very high confidence). The simulation of large-scale patterns of precipitation has improved somewhat since the AR4, although models continue to perform less well for precipitation than for surface temperature. Confidence in the representation of processes involving clouds and aerosols remains low. \{WGI SPM D.1, 7.2.3, 7.3.3, 7.6.2, 9.4, 9.5, 9.8, 10.3.1\}

The ability to simulate ocean thermal expansion, glaciers and ice sheets, and thus sea level, has improved since the AR4, but significant challenges remain in representing the dynamics of the Greenland and Antarctic ice sheets. This, together with advances in scientific understanding and capability, has resulted in improved sea level projections in this report, compared with the AR4. \{WGI SPM E.6, 9.1.3, 9.2, 9.4.2, 9.6, 9.8, 13.1, 13.4, 13.5\}

There is overall consistency between the projections from climate models in AR4 and AR5 for large-scale patterns of change and the magnitude of the uncertainty has not changed significantly, but new experiments and studies have led to a more complete and rigorous characterization of the uncertainty in long-term projections. \{WGI 12.4\} 


\section{Box 2.2 | The Representative Concentration Pathways}

The Representative Concentration Pathways (RCPs) describe four different 21st century pathways of greenhouse gas (GHG) emissions and atmospheric concentrations, air pollutant emissions and land use. The RCPs have been developed using Integrated Assessment Models (IAMs) as input to a wide range of climate model simulations to project their consequences for the climate system. These climate projections, in turn, are used for impacts and adaptation assessment. The RCPs are consistent with the wide range of scenarios in the mitigation literature assessed by WGIII28. The scenarios are used to assess the costs associated with emission reductions consistent with particular concentration pathways. The RCPs represent the range of GHG emissions in the wider literature well (Box 2.2, Figure 1); they include a stringent mitigation scenario (RCP2.6), two intermediate scenarios (RCP4.5 and RCP6.0), and one scenario with very high GHG emissions (RCP8.5). Scenarios without additional efforts to constrain emissions ('baseline scenarios') lead to pathways ranging between RCP6.0 and RCP8.5. RCP2.6 is representative of a scenario that aims to keep global warming likely below $2^{\circ} \mathrm{C}$ above pre-industrial temperatures. The majority of models indicate that scenarios meeting forcing levels similar to RCP2.6 are characterized by substantial net negative emissions ${ }^{29}$ by 2100 , on average around $2 \mathrm{GtCO}_{2} / \mathrm{yr}$. The land use scenarios of RCPs, together, show a wide range of possible futures, ranging from a net reforestation to further deforestation, consistent with projections in the full scenario literature. For air pollutants such as sulfur dioxide $\left(\mathrm{SO}_{2}\right)$, the $\mathrm{RCP}$ scenarios assume a consistent decrease in emissions as a consequence of assumed air pollution control and GHG mitigation policy (Box 2.2, Figure 1). Importantly, these future scenarios do not account for possible changes in natural forcings (e.g., volcanic eruptions) (see Box 1.1). \{WGI Box SPM.1, 6.4, 8.5.3, 12.3, Annex II, WGII 19, 21, WGIII 6.3.2, 6.3.6\}

The RCPs cover a wider range than the scenarios from the Special Report on Emissions Scenarios (SRES) used in previous assessments, as they also represent scenarios with climate policy. In terms of overall forcing, RCP8.5 is broadly comparable to the SRES A2/A1FI scenario, RCP6.0 to B2 and RCP4.5 to B1. For RCP2.6, there is no equivalent scenario in SRES. As a result, the differences in the magnitude of AR4 and AR5 climate projections are largely due to the inclusion of the wider range of emissions assessed. $\{W G I$ TS Box TS.6, 12.4.9\}
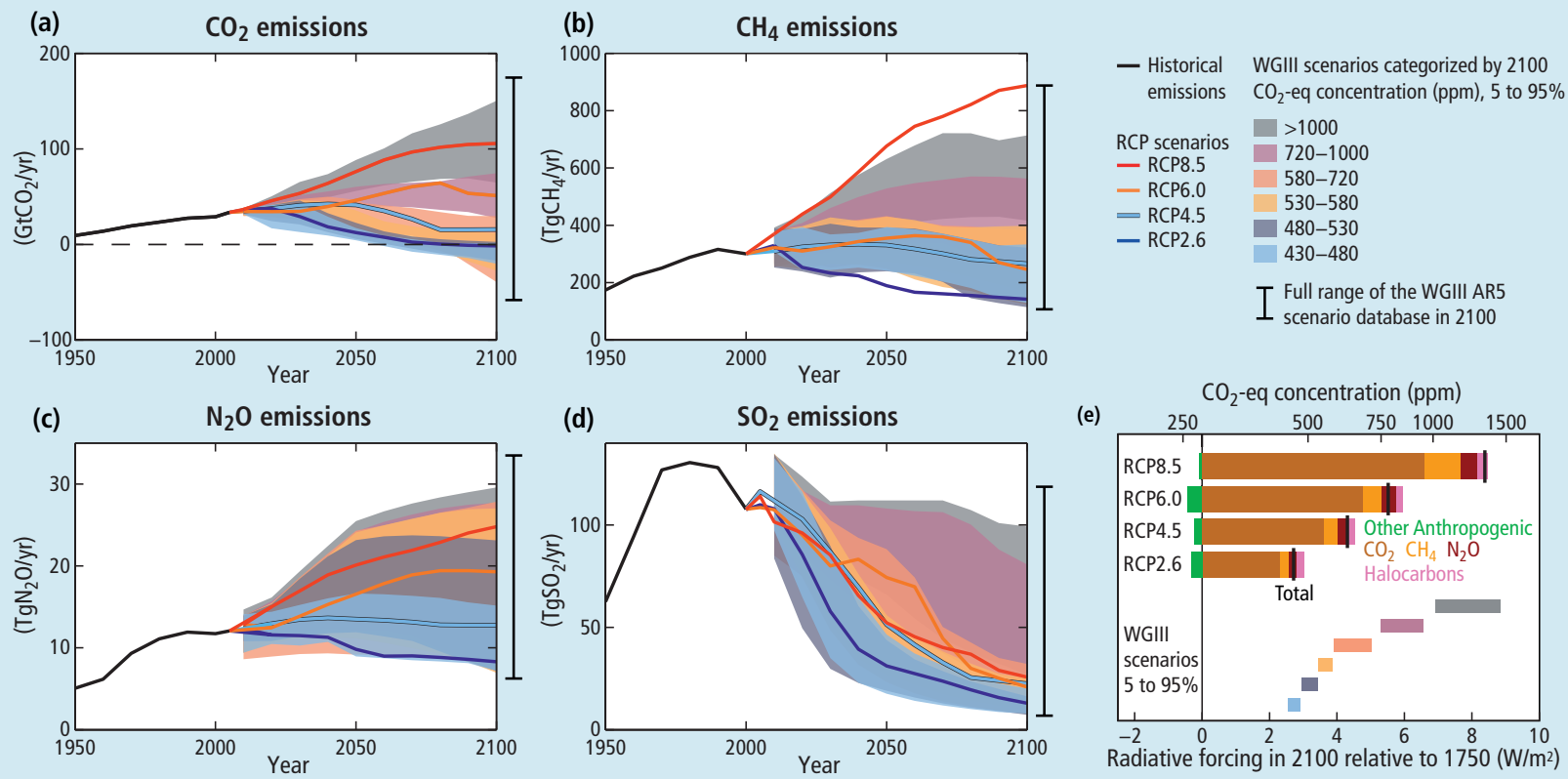

Box 2.2, Figure 1 | Emission scenarios and the resulting radiative forcing levels for the Representative Concentration Pathways (RCPs, lines) and the associated scenarios categories used in WGIII (coloured areas, see Table 3.1). Panels a to d show the emissions of carbon dioxide $\left(\mathrm{CO}_{2}\right)$, methane $\left(\mathrm{CH}_{4}\right)$, nitrous oxide $\left(\mathrm{N}_{2} \mathrm{O}\right)$ and sulfur dioxide $\left(\mathrm{SO}_{2}\right)$. Panel e shows future radiative forcing levels for the RCPs calculated using the simple carbon cycle climate model, Model for the Assessment of Greenhouse Gas Induced Climate Change (MAGICC), for the RCPs (per forcing agent) and for the WGIII scenario categories (total) \{WGI 8.2.2, 8.5.3, Figure 8.2, Annex II, WGIII Table SPM.1, Table 6.3\}. The WGIII scenario categories summarize the wide range of emission scenarios published in the scientific literature and are defined based on total $\mathrm{CO}_{2}$-equivalent concentrations (in ppm) in 2100 (Table 3.1). The vertical lines to the right of the panels (panel a-d) indicate the full range of the WGIII AR5 scenario database.

28 Roughly 300 baseline scenarios and 900 mitigation scenarios are categorized by $\mathrm{CO}_{2}$-equivalent concentration $\left(\mathrm{CO}_{2}\right.$-eq) by 2100 . The $\mathrm{CO}_{2}$-eq includes the forcing due to all GHGs (including halogenated gases and tropospheric ozone), aerosols and albedo change (see Glossary).

29 Net negative emissions can be achieved when more GHGs are sequestered than are released into the atmosphere (e.g., by using bio-energy in combination with carbon dioxide capture and storage). 
The methods used to estimate future impacts and risks resulting from climate change are described in Box 2.3. Modelled future impacts assessed in this report are generally based on climate-model projections using the RCPs, and in some cases, the older Special Report on Emissions Scenarios (SRES). \{WGI Box SPM.1, WGII 1.1, 1.3, 2.2-2.3, 19.6, 20.2, 21.3, 21.5, 26.2, Box CC-RC\}

Risk of climate-related impacts results from the interaction between climate-related hazards (including hazardous events and trends) and the vulnerability and exposure of human and natural systems. Alternative development paths influence risk by changing the likelihood of climatic events and trends, through their effects on GHGs, pollutants and land use, and by altering vulnerability and exposure. \{WGII SPM, 19.2.4, Figure 19-1, Box 19-2\}

Experiments, observations and models used to estimate future impacts and risks have improved since the AR4, with increasing understanding across sectors and regions. For example, an improved knowledge base has enabled expanded assessment of risks for human security and livelihoods and for the oceans. For some aspects of climate change and climate change impacts, uncertainty about future outcomes has narrowed. For others, uncertainty will persist. Some of the persistent uncertainties are grounded in the mechanisms that control the magnitude and pace of climate change. Others emerge from potentially complex interactions between the changing climate and the underlying vulnerability and exposure of people, societies and ecosystems. The combination of persistent uncertainty in key mechanisms plus the prospect of complex interactions motivates a focus on risk in this report. Because risk involves both probability and consequence, it is important to consider the full range of possible outcomes, including low-probability, high-consequence impacts that are difficult to simulate. \{WGII $2.1-2.4,3.6,4.3,11.3,12.6,19.2,19.6$, $21.3-21.5,22.4,25.3-25.4,25.11,26.2\}$

\subsection{Projected changes in the climate system}

Surface temperature is projected to rise over the 21st century under all assessed emission scenarios. It is very likely that heat waves will occur more often and last longer, and that extreme precipitation events will become more intense and frequent in many regions. The ocean will continue to warm and acidify, and global mean sea level to rise.

The projected changes in Section 2.2 are for 2081-2100 relative to 1986-2005, unless otherwise indicated.

\subsubsection{Air temperature}

The global mean surface temperature change for the period 20162035 relative to $1986-2005$ is similar for the four RCPs, and will likely be in the range $0.3^{\circ} \mathrm{C}$ to $0.7^{\circ} \mathrm{C}$ (medium confidence) ( $^{30}$. This range assumes no major volcanic eruptions or changes in some natural sources (e.g., methane $\left(\mathrm{CH}_{4}\right)$ and nitrous oxide $\left(\mathrm{N}_{2} \mathrm{O}\right)$ ), or unexpected changes in total solar irradiance. Future climate will depend on

\section{Box 2.3 | Models and Methods for Estimating Climate Change Risks, Vulnerability and Impacts}

Future climate-related risks, vulnerabilities and impacts are estimated in the AR5 through experiments, analogies and models, as in previous assessments. 'Experiments' involve deliberately changing one or more climate-system factors affecting a subject of interest to reflect anticipated future conditions, while holding the other factors affecting the subject constant. 'Analogies' make use of existing variations and are used when controlled experiments are impractical due to ethical constraints, the large area or long time required or high system complexity. Two types of analogies are used in projections of climate and impacts. Spatial analogies identify another part of the world currently experiencing similar conditions to those anticipated to be experienced in the future. Temporal analogies use changes in the past, sometimes inferred from paleo-ecological data, to make inferences about changes in the future. 'Models' are typically numerical simulations of real-world systems, calibrated and validated using observations from experiments or analogies, and then run using input data representing future climate. Models can also include largely descriptive narratives of possible futures, such as those used in scenario construction. Quantitative and descriptive models are often used together. Impacts are modelled, among other things, for water resources, biodiversity and ecosystem services on land, inland waters, the oceans and ice bodies, as well as for urban infrastructure, agricultural productivity, health, economic growth and poverty. \{WGII 2.2.1, 2.4.2, 3.4.1, 4.2.2, 5.4.1, 6.5, 7.3.1, 11.3.6, 13.2.2\}

Risks are evaluated based on the interaction of projected changes in the Earth system with the many dimensions of vulnerability in societies and ecosystems. The data are seldom sufficient to allow direct estimation of probabilities of a given outcome; therefore, expert judgment using specific criteria (large magnitude, high probability or irreversibility of impacts; timing of impacts; persistent vulnerability or exposure contributing to risks; or limited potential to reduce risks through adaptation or mitigation) is used to integrate the diverse information sources relating to the severity of consequences and the likelihood of occurrence into a risk evaluation, considering exposure and vulnerability in the context of specific hazards. \{WGII 11.3, 19.2, 21.1, 21.3-21.5, 25.3-25.4, 25.11, $26.2\}$ 

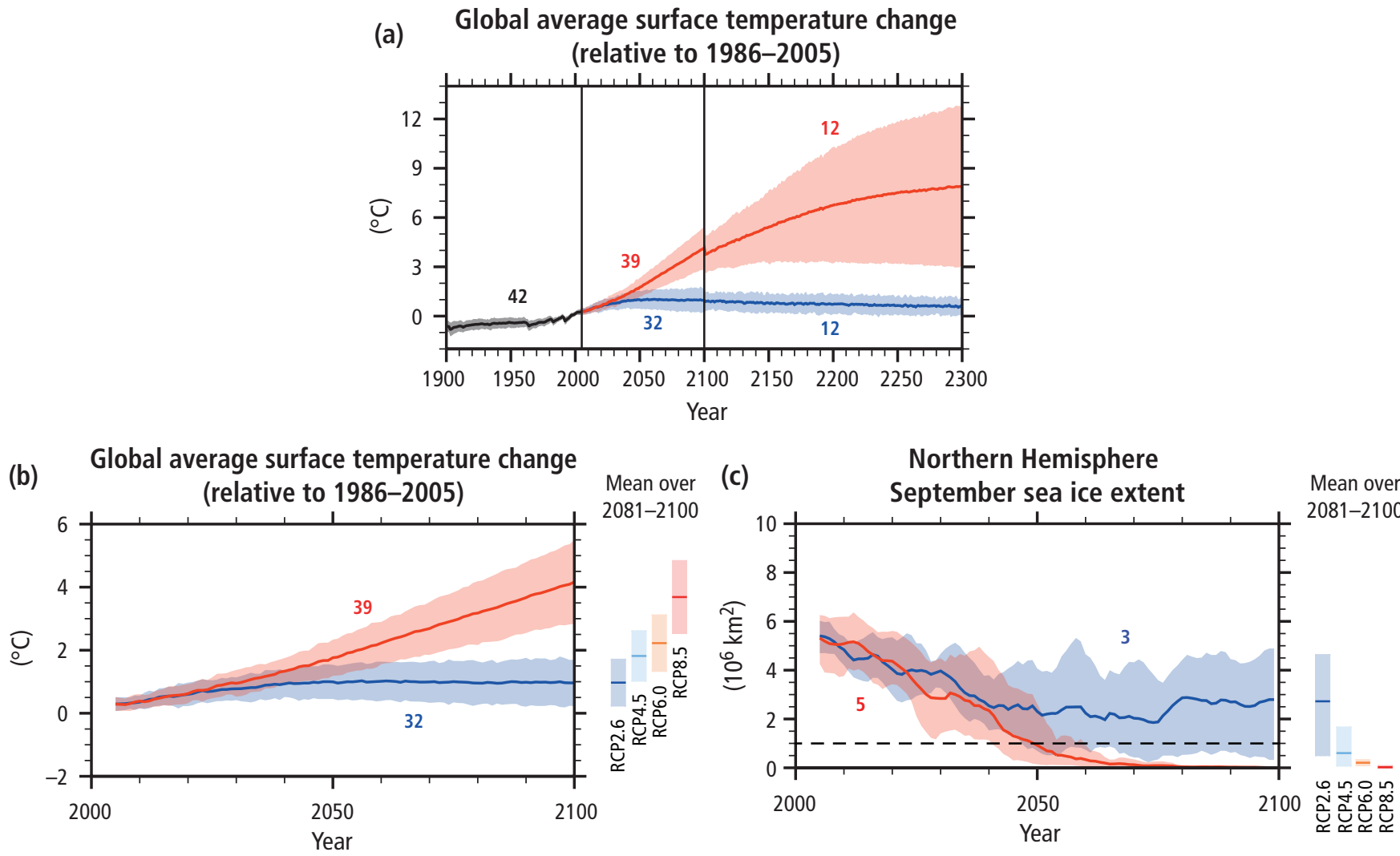

(d)

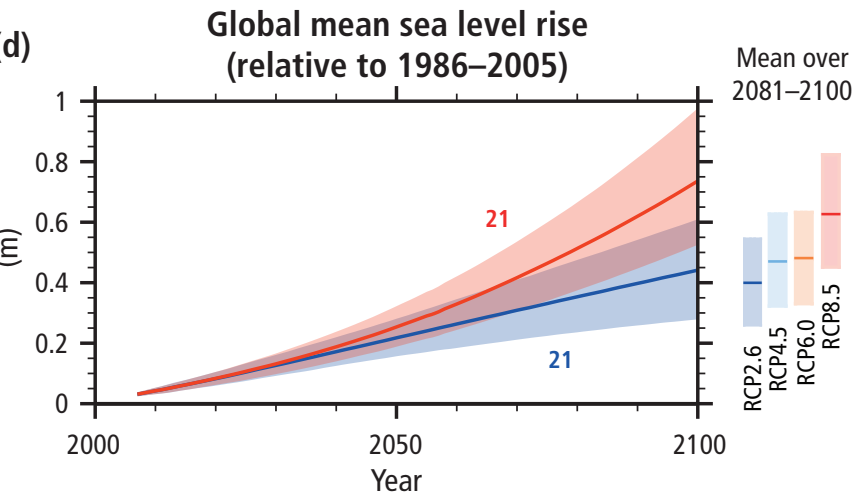

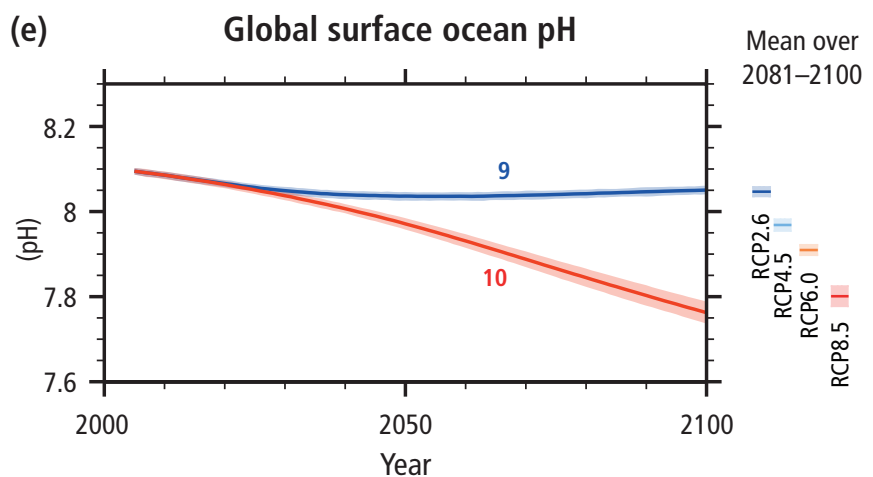

Figure 2.1 | (a) Time series of global annual change in mean surface temperature for the 1900-2300 period (relative to 1986-2005) from Coupled Model Intercomparison Project Phase 5 (CMIP5) concentration-driven experiments. Projections are shown for the multi-model mean (solid lines) and the 5 to $95 \%$ range across the distribution of individual models (shading). Grey lines and shading represent the CMIP5 historical simulations. Discontinuities at 2100 are due to different numbers of models performing the extension runs beyond the 21st century and have no physical meaning. (b) Same as (a) but for the 2006-2100 period (relative to 1986-2005). (c) Change in Northern Hemisphere September sea-ice extent ( 5 year running mean). The dashed line represents nearly ice-free conditions (i.e., when September sea-ice extent is less than $10^{6} \mathrm{~km}^{2}$ for at least five consecutive years). (d) Change in global mean sea level. (e) Change in ocean surface pH. For all panels, time series of projections and a measure of uncertainty (shading) are shown for scenarios RCP2.6 (blue) and RCP8.5 (red). The number of CMIP5 models used to calculate the multi-model mean is indicated. The mean and associated uncertainties averaged over the $2081-2100$ period are given for all RCP scenarios as coloured vertical bars on the right hand side of panels (b) to (e). For sea-ice extent (c), the projected mean and uncertainty (minimummaximum range) is only given for the subset of models that most closely reproduce the climatological mean state and the 1979-2012 trend in the Arctic sea ice. For sea level (d) based on current understanding (from observations, physical understanding and modelling), only the collapse of marine-based sectors of the Antarctic ice sheet, if initiated, could cause global mean sea level to rise substantially above the likely range during the 21 st century. However, there is medium confidence that this additional contribution would not exceed several tenths of a meter of sea level rise during the 21st century. \{WGI Figure SPM.7, Figure SPM.9, Figure 12.5, 6.4.4, 12.4.1, 13.4.4, 13.5.1\}

committed warming caused by past anthropogenic emissions, as well as future anthropogenic emissions and natural climate variability. By the mid-21st century, the magnitude of the projected climate change is substantially affected by the choice of emissions scenarios. Climate change continues to diverge among the scenarios through to 2100 and beyond (Table 2.1, Figure 2.1). The ranges provided for particular RCPs (Table 2.1), and those given below in Section 2.2, primarily arise from differences in the sensitivity of climate models to the imposed forcing. \{WGI SPM E.1, 11.3.2, 12.4.1\} 
Table 2.1 | Projected change in global mean surface temperature and global mean sea level rise for the mid- and late 21st century, relative to the 1986-2005 period. \{WGI Table SPM.2, 12.4.1, 13.5.1, Table 12.2, Table 13.5\}

\begin{tabular}{|c|c|c|c|c|c|}
\hline & \multirow[b]{2}{*}{ Scenario } & \multicolumn{2}{|c|}{ 2046-2065 } & \multicolumn{2}{|c|}{$2081-2100$} \\
\hline & & Mean & Likely range ${ }^{c}$ & Mean & Likely range ${ }^{c}$ \\
\hline \multirow{3}{*}{$\begin{array}{c}\text { Global Mean Surface } \\
\text { Temperature Change }\left({ }^{\circ} \mathrm{C}\right)^{a}\end{array}$} & $\mathrm{RCP} 2.6$ & 1.0 & 0.4 to 1.6 & 1.0 & 0.3 to 1.7 \\
\hline & RCP6.0 & 1.3 & 0.8 to 1.8 & 2.2 & 1.4 to 3.1 \\
\hline & RCP8.5 & 2.0 & 1.4 to 2.6 & 3.7 & 2.6 to 4.8 \\
\hline \multirow{4}{*}{ Global Mean Sea Level Rise $(\mathrm{m})^{\mathrm{b}}$} & RCP2.6 & 0.24 & 0.17 to 0.32 & 0.40 & 0.26 to 0.55 \\
\hline & RCP4.5 & 0.26 & 0.19 to 0.33 & 0.47 & 0.32 to 0.63 \\
\hline & $\mathrm{RCP6.0}$ & 0.25 & 0.18 to 0.32 & 0.48 & 0.33 to 0.63 \\
\hline & RCP8.5 & 0.30 & 0.22 to 0.38 & 0.63 & 0.45 to 0.82 \\
\hline
\end{tabular}

Notes:

a Based on the Coupled Model Intercomparison Project Phase 5 (CMIP5) ensemble; changes calculated with respect to the 1986-2005 period. Using Hadley Centre Climatic Research Unit Gridded Surface Temperature Data Set 4 (HadCRUT4) and its uncertainty estimate (5 to $95 \%$ confidence interval), the observed warming from $1850-1900$ to the reference period $1986-2005$ is 0.61 [0.55 to 0.67$]^{\circ} \mathrm{C}$. Likely ranges have not been assessed here with respect to earlier reference periods because methods are not generally available in the literature for combining the uncertainties in models and observations. Adding projected and observed changes does not account for potential effects of model biases compared to observations, and for natural internal variability during the observational reference period. \{WGI 2.4.3, 11.2.2, 12.4.1, Table 12.2, Table 12.3\}

${ }^{\mathrm{b}}$ Based on 21 CMIP5 models; changes calculated with respect to the 1986-2005 period. Based on current understanding (from observations, physical understanding and modelling), only the collapse of marine-based sectors of the Antarctic ice sheet, if initiated, could cause global mean sea level to rise substantially above the likely range during the 21 st century. There is medium confidence that this additional contribution would not exceed several tenths of a meter of sea level rise during the 21 st century.

c Calculated from projections as 5 to $95 \%$ model ranges. These ranges are then assessed to be likely ranges after accounting for additional uncertainties or different levels of confidence in models. For projections of global mean surface temperature change in 2046-2065, confidence is medium, because the relative importance of natural internal variability, and uncertainty in non-greenhouse gas forcing and response, are larger than for the 2081-2100 period. The likely ranges for 2046-2065 do not take into account the possible influence of factors that lead to the assessed range for near term (2016-2035) change in global mean surface temperature that is lower than the 5 to $95 \%$ model range, because the influence of these factors on longer term projections has not been quantified due to insufficient scientific understanding. \{WGI 11.3.1\}

${ }^{d}$ Calculated from projections as 5 to $95 \%$ model ranges. These ranges are then assessed to be likely ranges after accounting for additional uncertainties or different levels of confidence in models. For projections of global mean sea level rise confidence is medium for both time horizons.

Relative to $1850-1900$, global surface temperature change for the end of the 21st century (2081-2100) is projected to likely exceed $1.5^{\circ} \mathrm{C}$ for RCP4.5, RCP6.0 and RCP8.5 (high confidence). Warming is likely to exceed $2^{\circ} \mathrm{C}$ for RCP6.0 and RCP8.5 (high confidence), more likely than not to exceed $2^{\circ} \mathrm{C}$ for RCP4.5 (medium confidence), but unlikely to exceed $2^{\circ} \mathrm{C}$ for RCP2.6 (medium confidence). \{WGI SPM E.1, 12.4.1, Table 12.3\}

The Arctic region will continue to warm more rapidly than the global mean (Figure 2.2) (very high confidence). The mean warming over land will be larger than over the ocean (very high confidence) and larger than global average warming (Figure 2.2). \{WGI SPM E.1, 11.3.2, 12.4.3, 14.8.2\}

It is virtually certain that there will be more frequent hot and fewer cold temperature extremes over most land areas on daily and seasonal timescales, as global mean surface temperature increases. It is very likely that heat waves will occur with a higher frequency and longer duration. Occasional cold winter extremes will continue to occur. \{WGI SPM E.1, 12.4.3\}

\subsubsection{Water cycle}

Changes in precipitation in a warming world will not be uniform. The high latitudes and the equatorial Pacific are likely to experience an increase in annual mean precipitation by the end of this century under the RCP8.5 scenario. In many mid-latitude and subtropical dry regions, mean precipitation will likely decrease, while in many mid-latitude wet regions, mean precipitation will likely increase under the RCP8.5 scenario (Figure 2.2). \{WGI SPM E.2, 7.6.2, 12.4.5, 14.3.1, 14.3.5\}

Extreme precipitation events over most mid-latitude land masses and over wet tropical regions will very likely become more intense and more frequent as global mean surface temperature increases. $\{W G \mid$ SPM E.2, 7.6.2, 12.4.5\}

Globally, in all RCPs, it is likely that the area encompassed by monsoon systems will increase and monsoon precipitation is likely to intensify and El Niño-Southern Oscillation (ENSO) related precipitation variability on regional scales will likely intensify. \{WGI SPM E.2, 14.2, 14.4\}

\subsubsection{Ocean, cryosphere and sea level}

The global ocean will continue to warm during the 21st century. The strongest ocean warming is projected for the surface in tropical and Northern Hemisphere subtropical regions. At greater depth the warming will be most pronounced in the Southern Ocean (high confidence). \{WGI SPM E.4, 6.4.5, 12.4.7\}

It is very likely that the Atlantic Meridional Overturning Circulation (AMOC) will weaken over the 21st century, with best estimates and model ranges for the reduction of $11 \%$ (1 to $24 \%$ ) for 
RCP2.6

RCP8.5

(a)

Change in average surface temperature (1986-2005 to 2081-2100)

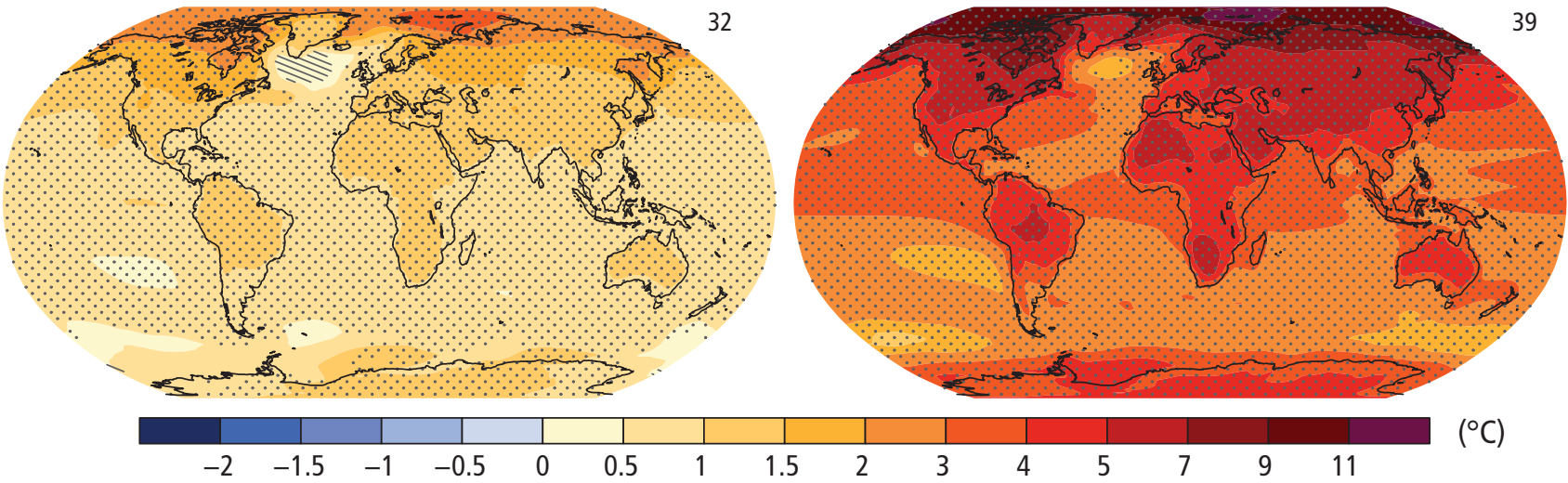

(b)

Change in average precipitation (1986-2005 to 2081-2100)
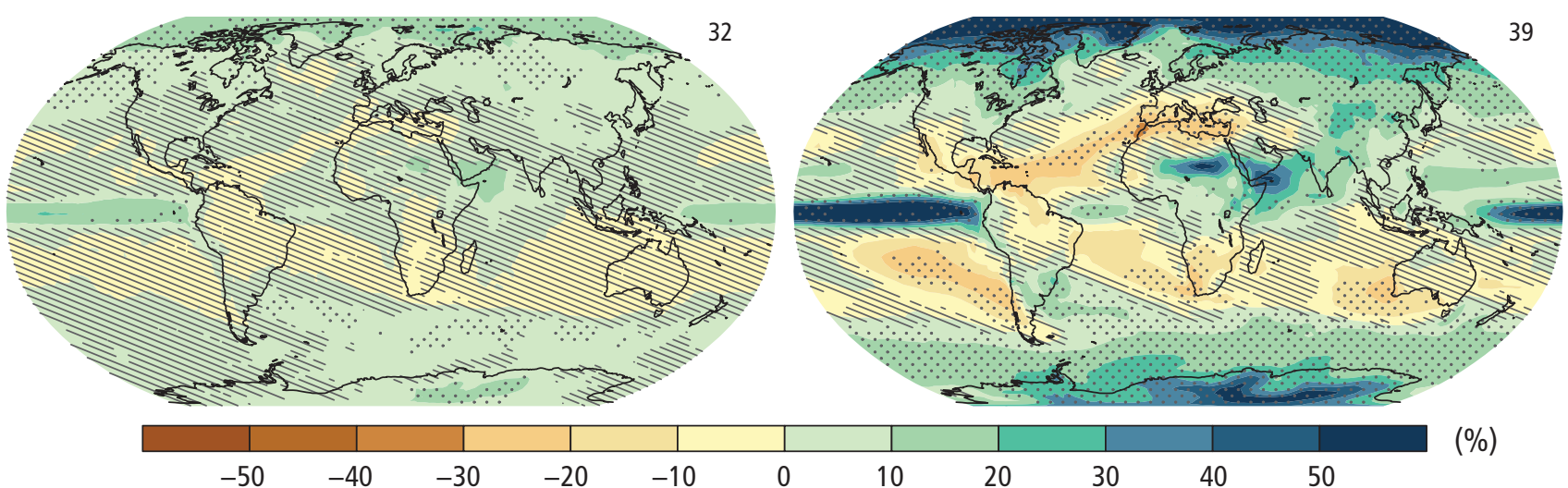

(\%)

(c)

Change in average sea level (1986-2005 to 2081-2100)

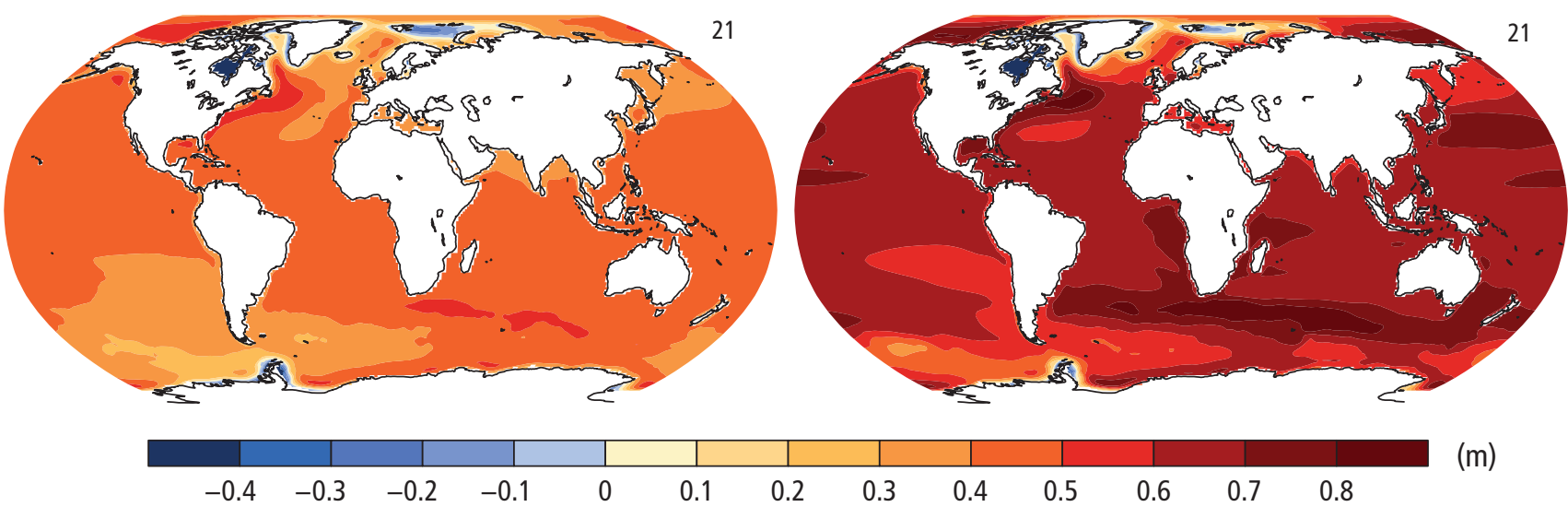

Figure 2.2 | Coupled Model Intercomparison Project Phase 5 (CMIP5) multi-model mean projections (i.e., the average of the model projections available) for the $2081-2100$ period under the RCP2.6 (left) and RCP8.5 (right) scenarios for (a) change in annual mean surface temperature and (b) change in annual mean precipitation, in percentages, and (c) change in average sea level. Changes are shown relative to the 1986-2005 period. The number of CMIP5 models used to calculate the multi-model mean is indicated in the upper right corner of each panel. Stippling (dots) on (a) and (b) indicates regions where the projected change is large compared to natural internal variability (i.e., greater than two standard deviations of internal variability in 20-year means) and where $90 \%$ of the models agree on the sign of change. Hatching (diagonal lines) on (a) and (b) shows regions where the projected change is less than one standard deviation of natural internal variability in 20-year means. \{WGI Figure SPM.8, Figure 13.20, Box 12.1\} 
the RCP2.6 scenario, 34\% (12 to 54\%) for the RCP8.5. Nevertheless, it is very unlikely that the AMOC will undergo an abrupt transition or collapse in the 21st century. \{WGI SPM E.4, 12.4.7.2\}

Year-round reductions in Arctic sea ice are projected for all RCP scenarios. The subset of models that most closely reproduce the observations ${ }^{31}$ project that a nearly ice-free Arctic Ocean ${ }^{32}$ in September is likely for RCP8.5 before mid-century (medium confidence) (Figure 2.1). In the Antarctic, a decrease in sea ice extent and volume is projected with low confidence. \{WGI SPM E.5, 12.4.6.1\}

The area of Northern Hemisphere spring snow cover is likely to decrease by $7 \%$ for RCP2.6 and by $25 \%$ in RCP8.5 by the end of the 21 st century for the multi-model average (medium confidence). \{WGI SPM E.5, 12.4.6\}

It is virtually certain that near-surface permafrost extent at high northern latitudes will be reduced as global mean surface temperature increases. The area of permafrost near the surface (upper $3.5 \mathrm{~m}$ ) is likely to decrease by $37 \%$ (RCP2.6) to $81 \%$ (RCP8.5) for the multi-model average (medium confidence). \{WGI SPM E.5, 12.4.6\}

The global glacier volume, excluding glaciers on the periphery of Antarctica (and excluding the Greenland and Antarctic ice sheets), is projected to decrease by 15 to $55 \%$ for RCP2.6 and by 35 to $85 \%$ for RCP8.5 (medium confidence). \{WGI SPM E.5, 13.4.2, 13.5.1\}

Global mean sea level will continue to rise during the 21 st century (Table 2.1, Figure 2.1). There has been significant improvement in understanding and projection of sea level change since the AR4. Under all RCP scenarios, the rate of sea level rise will very likely exceed the observed rate of 2.0 [1.7-2.3] mm/yr during 1971-2010, with the rate of rise for RCP8.5 during 2081-2100 of 8 to $16 \mathrm{~mm} / \mathrm{yr}$ (medium confidence). \{WGI SPM B4, SPM E.6, 13.5.1\}

Sea level rise will not be uniform across regions. By the end of the 21st century, it is very likely that sea level will rise in more than about $95 \%$ of the ocean area. Sea level rise depends on the pathway of $\mathrm{CO}_{2}$ emissions, not only on the cumulative total; reducing emissions earlier rather than later, for the same cumulative total, leads to a larger mitigation of sea level rise. About $70 \%$ of the coastlines worldwide are projected to experience sea level change within $\pm 20 \%$ of the global mean (Figure 2.2). It is very likely that there will be a significant increase in the occurrence of future sea level extremes in some regions by 2100. \{WGI SPM E.6, TS 5.7.1, 12.4.1, 13.4.1, 13.5.1, 13.6.5, 13.7.2, Table 13.5\}

\subsubsection{Carbon cycle and biogeochemistry}

Ocean uptake of anthropogenic $\mathrm{CO}_{2}$ will continue under all four RCPs through to 2100, with higher uptake for higher concentration pathways (very high confidence). The future evolution of the land carbon uptake is less certain. A majority of models projects a continued land carbon uptake under all RCPs, but some models simulate a land carbon loss due to the combined effect of climate change and land use change. $\{W G I$ SPM E.7, 6.4.2, 6.4.3\}

Based on Earth System Models, there is high confidence that the feedback between climate change and the carbon cycle will amplify global warming. Climate change will partially offset increases in land and ocean carbon sinks caused by rising atmospheric $\mathrm{CO}_{2}$. As a result more of the emitted anthropogenic $\mathrm{CO}_{2}$ will remain in the atmosphere, reinforcing the warming. \{WGI SPM E.7, 6.4.2, 6.4.3\}

Earth System Models project a global increase in ocean acidification for all RCP scenarios by the end of the 21st century, with a slow recovery after mid-century under RCP2.6. The decrease in surface ocean $\mathrm{pH}$ is in the range of 0.06 to 0.07 (15 to $17 \%$ increase in acidity) for RCP2.6, 0.14 to 0.15 (38 to $41 \%$ ) for RCP4.5, 0.20 to 0.21 (58 to $62 \%$ ) for RCP6.0, and 0.30 to 0.32 (100 to $109 \%$ ) for RCP8.5 (Figure 2.1). \{WGI SPM E.7, 6.4.4\}

It is very likely that the dissolved oxygen content of the ocean will decrease by a few percent during the 21st century in response to surface warming, predominantly in the subsurface mid-latitude oceans. There is no consensus on the future volume of low oxygen waters in the open ocean because of large uncertainties in potential biogeochemical effects and in the evolution of tropical ocean dynamics. \{WGI TS 5.6, 6.4.5, WGII TS B-2, 6.1\}

\subsubsection{Climate system responses}

Climate system properties that determine the response to external forcing have been estimated both from climate models and from analysis of past and recent climate change. The equilibrium climate sensitivity (ECS) $)^{33}$ is likely in the range $1.5^{\circ} \mathrm{C}$ to $4.5^{\circ} \mathrm{C}$, extremely unlikely less than $1^{\circ} \mathrm{C}$, and very unlikely greater than $6^{\circ} \mathrm{C}$. \{WGI SPM D.2, TS TFE.6, 10.8.1, 10.8.2, 12.5.4, Box 12.2\}

Cumulative emissions of $\mathrm{CO}_{2}$ largely determine global mean surface warming by the late 21 st century and beyond. Multiple lines of evidence indicate a strong and consistent near-linear relationship across all scenarios considered between net cumulative $\mathrm{CO}_{2}$ emissions (including the impact of $\mathrm{CO}_{2}$ removal) and projected global temperature change to the year 2100 (Figure 2.3). Past emissions and observed warming support this relationship within uncertainties. Any given level of warming is associated with a range of cumulative $\mathrm{CO}_{2}$ emissions (depending on non- $\mathrm{CO}_{2}$ drivers), and therefore, for example, higher emissions in earlier decades imply lower emissions later. \{WGI SPM E.8, TS TFE.8, 12.5.4\}

The global mean peak surface temperature change per trillion tonnes of carbon $(1000 \mathrm{GtC})$ emitted as $\mathrm{CO}_{2}$ is likely in the range of $0.8^{\circ} \mathrm{C}$ to $2.5^{\circ} \mathrm{C}$. This quantity, called the transient climate response to cumulative carbon emissions (TCRE), is supported by both modelling and observational evidence and applies to cumulative emissions up to about 2000 GtC. \{WGI SPM D.2, TS TFE.6, 12.5.4, Box 12.2\}

\footnotetext{
Climatological mean state and the 1979-2012 trend in Arctic sea-ice extent.

32 When sea-ice extent is less than one million $\mathrm{km}^{2}$ for at least five consecutive years.

33 Defined as the equilibrium global average surface warming following a doubling of $\mathrm{CO}_{2}$ concentration (relative to pre-industrial). 


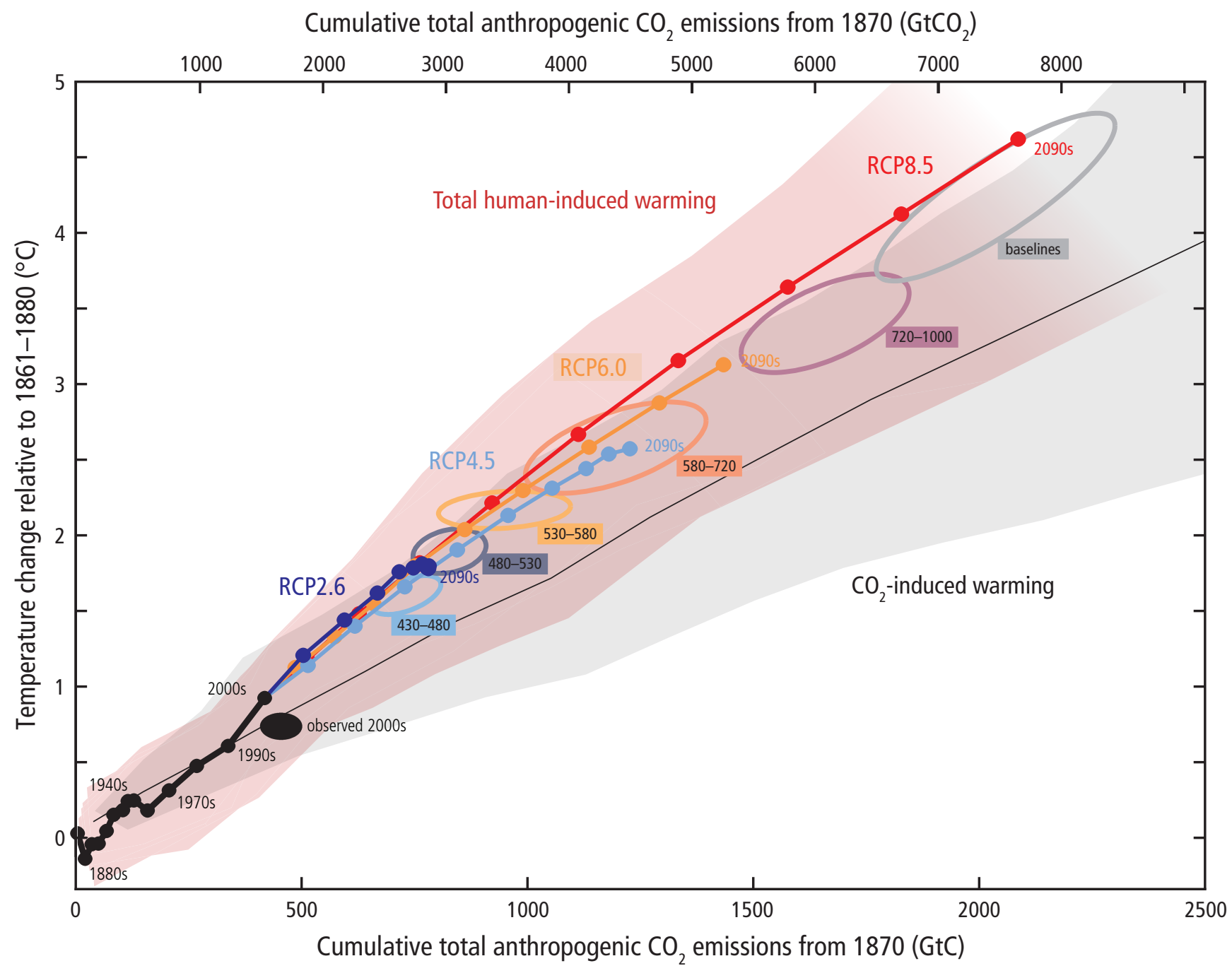

Figure 2.3 Global mean surface temperature increase as a function of cumulative total global carbon dioxide $\left(\mathrm{CO}_{2}\right)$ emissions from various lines of evidence. Multi-model results from a hierarchy of climate carbon-cycle models for each Representative Concentration Pathway (RCP) until 2100 are shown (coloured lines). Model results over the historical period (1860 to 2010) are indicated in black. The coloured plume illustrates the multi-model spread over the four RCP scenarios and fades with the decreasing number of available models in RCP8.5. Dots indicate decadal averages, with selected decades labelled. Ellipses show total anthropogenic warming in 2100 versus cumulative $\mathrm{CO}_{2}$ emissions from 1870 to 2100 from a simple climate model (median climate response) under the scenario categories used in WGIII. Temperature values are always given relative to the 1861-1880 period, and emissions are cumulative since 1870. Black filled ellipse shows observed emissions to 2005 and observed temperatures in the decade 2000-2009 with associated uncertainties. \{WGI SPM E.8, TS TFE.8, Figure 1, TS.SM.10, 12.5.4, Figure 12.45, WGIII Table SPM.1, Table 6.3\}

Warming caused by $\mathrm{CO}_{2}$ emissions is effectively irreversible over multi-century timescales unless measures are taken to remove $\mathrm{CO}_{2}$ from the atmosphere. Ensuring $\mathrm{CO}_{2}$-induced warming remains likely less than $2{ }^{\circ} \mathrm{C}$ requires cumulative $\mathrm{CO}_{2}$ emissions from all anthropogenic sources to remain below about $3650 \mathrm{GtCO}_{2}(1000 \mathrm{GtC})$, over half of which were already emitted by 2011. \{WGI SPM E.8, TS TFE.8, 12.5.2, 12.5.3, 12.5.4\}

Multi-model results show that limiting total human-induced warming (accounting for both $\mathrm{CO}_{2}$ and other human influences on climate) to less than $2^{\circ} \mathrm{C}$ relative to the period $1861-1880$ with a probability of $>66 \%$ would require total $\mathrm{CO}_{2}$ emissions from all anthropogenic sources since 1870 to be limited to about $2900 \mathrm{GtCO}_{2}$ when accounting for non- $\mathrm{CO}_{2}$ forcing as in the RCP2.6 scenario, with a range of 2550 to $3150 \mathrm{GtCO}_{2}$ arising from variations in non- $\mathrm{CO}_{2}$ climate drivers across the scenarios considered by WGIII (Table 2.2). About 1900 [1650 to
2150] $\mathrm{GtCO}_{2}$ were emitted by 2011, leaving about $1000 \mathrm{GtCO}_{2}$ to be consistent with this temperature goal. Estimated total fossil carbon reserves exceed this remaining amount by a factor of 4 to 7 , with resources much larger still. \{WGI SPM E.8, TS TFE.8, Figure 1, TS.SM.10, 12.5.4, Figure 12.45, WGIII Table SPM.1, Table 6.3, Table 7.2\} 
Table 2.2 Cumulative carbon dioxide $\left(\mathrm{CO}_{2}\right)$ emission consistent with limiting warming to less than stated temperature limits at different levels of probability, based on different lines of evidence. $\{W G / 12.5 .4$, WGIII 6\}

Cumulative $\mathrm{CO}_{2}$ emissions from 1870 in $\mathrm{GtCO}_{2}$

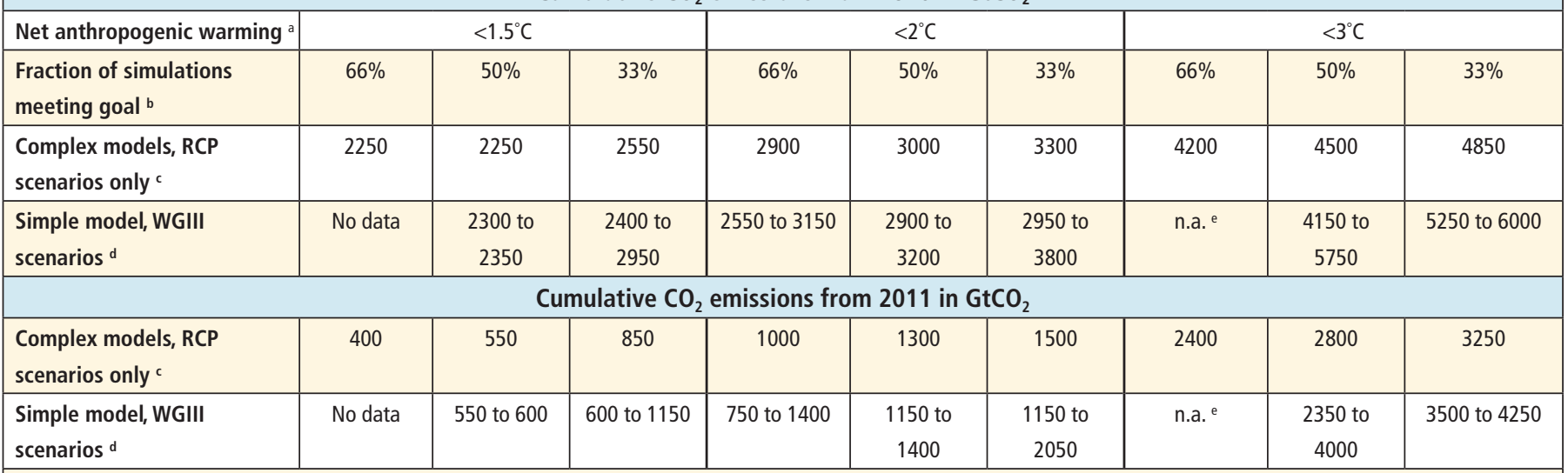

Total fossil carbon available in $2011^{\mathrm{f}}: 3670$ to $7100 \mathrm{GtCO}_{2}$ (reserves) and 31300 to $50050 \mathrm{GtCO}_{2}$ (resources)

Notes:

a Warming due to $\mathrm{CO}_{2}$ and non- $\mathrm{CO}_{2}$ drivers. Temperature values are given relative to the 1861-1880 base period.

${ }^{\mathrm{b}}$ Note that the $66 \%$ range in this table should not be equated to the likelihood statements in Table SPM.1 and Table 3.1 and WGIII Table SPM.1. The assessment in these latter tables is not only based on the probabilities calculated for the full ensemble of scenarios in WGIII using a single climate model, but also the assessment in WGI of the uncertainty of the temperature projections not covered by climate models.

'Cumulative $\mathrm{CO}_{2}$ emissions at the time the temperature threshold is exceeded that are required for $66 \%, 50 \%$ or $33 \%$ of the Coupled Model Intercomparison Project Phase 5 (CMIP5) complex models Earth System Model (ESM) and Earth System Models of Intermediate Complexity (EMIC) simulations, assuming non- $\mathrm{CO}_{2}$ forcing follows the RCP8.5 scenario. Similar cumulative emissions are implied by other RCP scenarios. For most scenario-threshold combinations, emissions and warming continue after the threshold

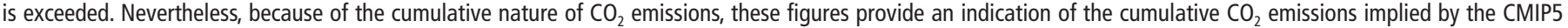
model simulations under RCP-like scenarios. Values are rounded to the nearest 50.

${ }^{d}$ Cumulative $\mathrm{CO}_{2}$ emissions at the time of peak warming from WGIII scenarios for which a fraction of greater than $66 \%$ (66 to $\left.100 \%\right)$, greater than $50 \%$ (50 to $\left.66 \%\right)$ or greater than $33 \%$ (33 to 50\%) of climate simulations keep global mean temperature increase to below the stated threshold. Ranges indicate the variation in cumulative $\mathrm{CO}_{2}$ emissions arising from differences in non- $\mathrm{CO}_{2}$ drivers across the WGIII scenarios. The fraction of climate simulations for each scenario is derived from a 600-member parameter ensemble of a simple carbon-cycle climate model, Model for the Assessment of Greenhouse Gas Induced Climate Change (MAGICC), in a probabilistic mode. Parameter and scenario uncertainty are explored in this ensemble. Structural uncertainties cannot be explored with a single model set-up. Ranges show the impact of scenario uncertainty, with $80 \%$ of scenarios giving cumulative $\mathrm{CO}_{2}$ emissions within the stated range for the given fraction of simulations. Simple model estimates are constrained by observed changes over the past century, do not account for uncertainty in model structure and may omit some feedback processes: they are hence slightly higher than the CMIP5 complex models estimates. Values are rounded to the nearest 50.

e The numerical results for the cumulative $\mathrm{CO}_{2}$ emissions for staying below $3^{\circ} \mathrm{C}$ with greater than $66 \%(66$ to $100 \%)$ is greatly influenced by a large number of scenarios that would also meet the $2^{\circ} \mathrm{C}$ objective and therefore not comparable with numbers provided for the other temperature threshold.

${ }^{f}$ Reserves are quantities able to be recovered under existing economic and operating conditions; resources are those where economic extraction is potentially feasible. \{WGIII Table 7.2\}

\subsection{Future risks and impacts caused by a changing climate}

Climate change will amplify existing risks and create new risks for natural and human systems. Risks are unevenly distributed and are generally greater for disadvantaged people and communities in countries at all levels of development. Increasing magnitudes of warming increase the likelihood of severe, pervasive and irreversible impacts for people, species and ecosystems. Continued high emissions would lead to mostly negative impacts for biodiversity, ecosystem services and economic development and amplify risks for livelihoods and for food and human security.

Risk of climate-related impacts results from the interaction of climate-related hazards (including hazardous events and trends) with the vulnerability and exposure of human and natural systems, including their ability to adapt. Rising rates and magnitudes of warming and other changes in the climate system, accompanied by ocean acidification, increase the risk of severe, pervasive, and in some cases, irreversible detrimental impacts. Future climate change will amplify existing climate-related risks and create new risks. \{WGII SPM B, Figure SPM.1\}

Key risks are potentially severe impacts relevant to understanding dangerous anthropogenic interference with the climate system. Risks are considered key due to high hazard or high vulnerability of societies and systems exposed, or both. Their identification is based on large magnitude or high probability of impacts; irreversibility or timing of impacts; persistent vulnerability or exposure; or limited potential to reduce risks. Some risks are particularly relevant for individual regions (Figure 2.4), while others are global (Table 2.3). For risk assessment it is important to evaluate the widest possible range of impacts, including low-probability outcomes with large consequences. Risk levels often increase with temperature (Box 2.4) and are sometimes more directly linked to other dimensions of climate change, such as the rate of warming, as well as the magnitudes and rates of ocean acidification and sea level rise (Figure 2.5). \{WGII SPM A-3, SPM B-1\} 


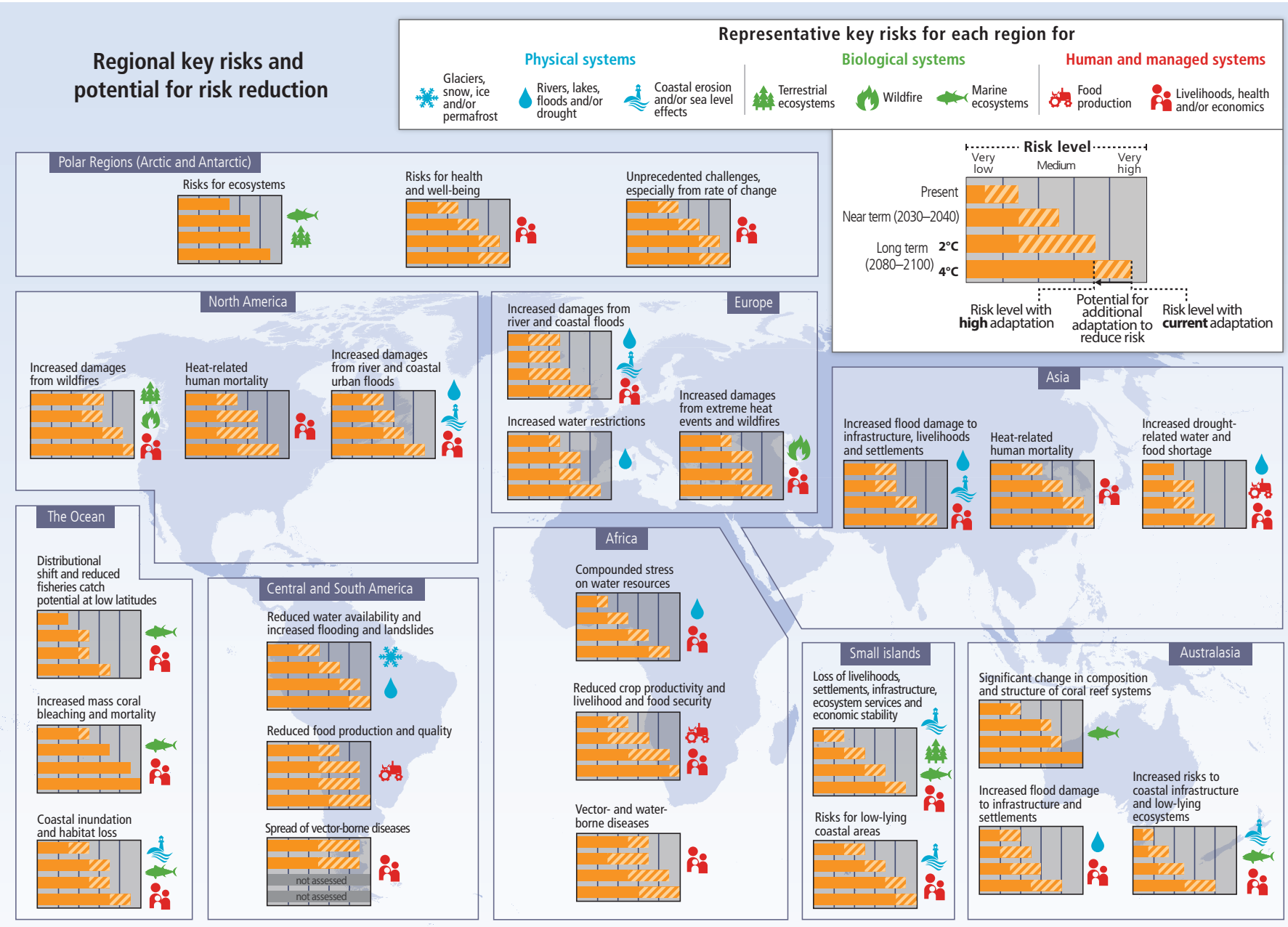

Figure 2.4 | Representative key risks for each region, including the potential for risk reduction through adaptation and mitigation, as well as limits to adaptation. Identification of key risks was based on expert judgment using the following specific criteria: large magnitude, high probability or irreversibility of impacts; timing of impacts; persistent vulnerability or exposure contributing to risks; or limited potential to reduce risks through adaptation or mitigation. Risk levels are assessed as very low, low, medium, high or very high for three timeframes: the present, near term (here, for 2030-2040) and long term (here, for 2080-2100). In the near term, projected levels of global mean temperature increase do not diverge substantially across different emission scenarios. For the long term, risk levels are presented for two possible futures $\left(2^{\circ} \mathrm{C}\right.$ and $4^{\circ} \mathrm{C}$ global mean temperature increase above pre-industrial levels). For each time frame, risk levels are indicated for a continuation of current adaptation and assuming high levels of current or future adaptation. Risk levels are not necessarily comparable, especially across regions. \{WGII SPM Assessment Box SPM.2 Table 1\}

\section{Key risks that span sectors and regions include the following (high confidence) \{WGII SPM B-1\}:}

1. Risk of severe ill-health and disrupted livelihoods resulting from storm surges, sea level rise and coastal flooding; inland flooding in some urban regions; and periods of extreme heat.

2. Systemic risks due to extreme weather events leading to breakdown of infrastructure networks and critical services.

3. Risk of food and water insecurity and loss of rural livelihoods and income, particularly for poorer populations.

4. Risk of loss of ecosystems, biodiversity and ecosystem goods, functions and services.
The overall risks of future climate change impacts can be reduced by limiting the rate and magnitude of climate change, including ocean acidification. Some risks are considerable even at $1^{\circ} \mathrm{C}$ global mean temperature increase above pre-industrial levels. Many global risks are high to very high for global temperature increases of $4^{\circ} \mathrm{C}$ or more (see Box 2.4). These risks include severe and widespread impacts on unique and threatened systems, the extinction of many species, large risks to food security and compromised normal human activities, including growing food or working outdoors in some areas for parts of the year, due to the combination of high temperature and humidity (high confidence). The precise levels of climate change sufficient to trigger abrupt and irreversible change remain uncertain, but the risk associated with crossing such thresholds in the earth system or in interlinked human and natural systems increases with rising temperature (medium confidence). \{WGII SPM B-1\} 
(a) Risk for terrestrial and freshwater species impacted by the rate of warming

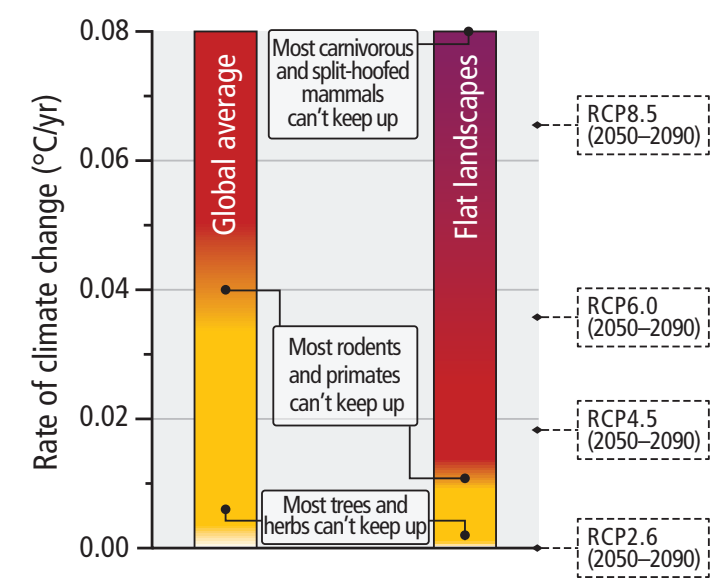

\section{Increasing risk from RCP2.6 to RCP8.5}

(b) Risk for marine species impacted by ocean acidification only, or additionally by warming extremes

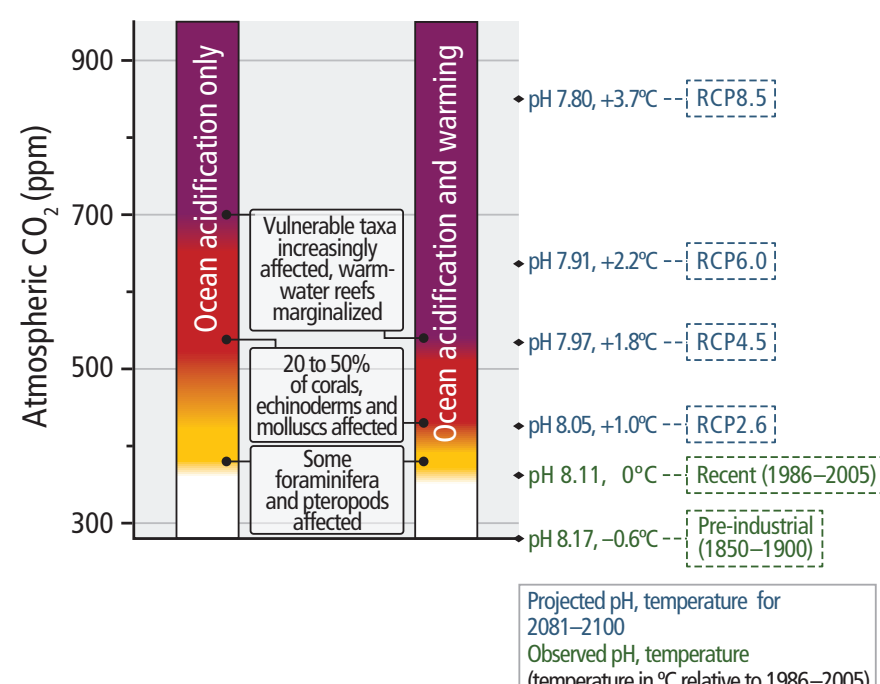

Observed $\mathrm{pH}$, temperature (c) Risk for coastal human and natural systems impacted by sea level rise

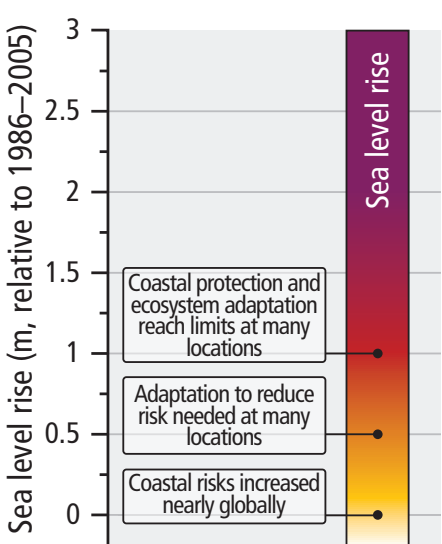
Medium $\mathrm{CO}_{2}$ ـ $2081-2100 \quad 2300$ The few available projection
for 2300 likely underestimate Antarctic ice sheet contributio

(temperature in ${ }^{\circ} \mathrm{C}$ relative to $1986-2005$ )

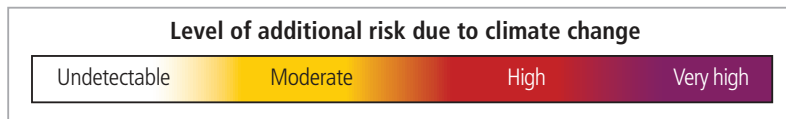

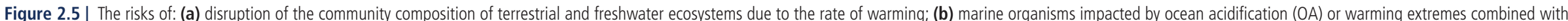

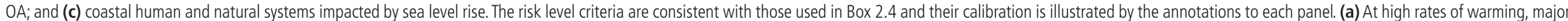

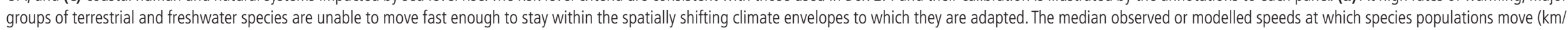

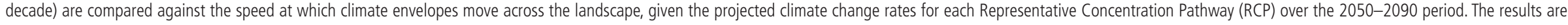

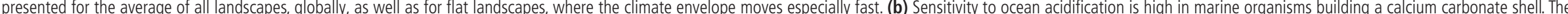

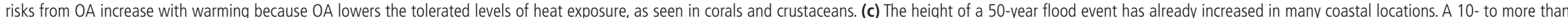

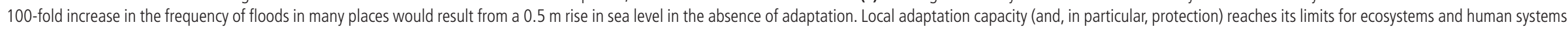
in many places under a $1 \mathrm{~m}$ sea level rise. (2.2.4, Table 2.1, Figure 2.8) \{WGI 3.7.5, 3.8, 6.4.4, Figure 13.25, WGIl Figure SPM.5, Figure 4-5, Figure 6-10, Box CC-OA, 4.4.2.5, 5.2, 5.3-5.5, 5.4.4, 5.5.6, 6.3\} 
Adaptation can substantially reduce the risks of climate change impacts, but greater rates and magnitude of climate change increase the likelihood of exceeding adaptation limits (high confidence). The potential for adaptation, as well as constraints and limits to adaptation, varies among sectors, regions, communities and ecosystems. The scope for adaptation changes over time and is closely linked to socio-economic development pathways and circumstances. See Figure 2.4 and Table 2.3, along with Topics 3 and 4. \{WGII SPM B, SPM C, TS B, TS C\}

\subsubsection{Ecosystems and their services in the oceans, along coasts, on land and in freshwater}

Risks of harmful impacts on ecosystems and human systems increase with the rates and magnitudes of warming, ocean acidification, sea level rise and other dimensions of climate change (high confidence). Future risk is indicated to be high by the observation that natural global climate change at rates lower than current anthropogenic climate change caused significant ecosystem shifts and species extinctions during the past millions of years on land and in the oceans (high confidence). Many plant and animal species will be unable to adapt locally or move fast enough during the 21 st century to track suitable climates under mid- and high range rates of climate change (RCP4.5, RCP6.0 and RCP8.5) (medium confidence) (Figure 2.5a). Coral reefs and polar ecosystems are highly vulnerable. \{WGII SPM A-1, SPM B-2, 4.3-4, 5.4, 6.1, 6.3, 6.5, 25.6, 26.4, 29.4, Box CC-CR, Box CC-MB, Box CC-RF\}

A large fraction of terrestrial, freshwater and marine species faces increased extinction risk due to climate change during and beyond the 21st century, especially as climate change interacts with other stressors (high confidence). Extinction risk is increased relative to pre-industrial and present periods, under all RCP scenarios, as a result of both the magnitude and rate of climate change (high confidence). Extinctions will be driven by several climate-associated drivers (warming, sea-ice loss, variations in precipitation, reduced river flows, ocean acidification and lowered ocean oxygen levels) and the interactions among these drivers and their interaction with simultaneous habitat modification, over-exploitation of stocks, pollution, eutrophication and invasive species (high confidence). \{WGII SPM B-2, 4.3-4.4, 6.1, 6.3, 6.5, 25.6, 26.4, Box CC-RF, Box CC-MB\}

Global marine species redistribution and marine biodiversity reduction in sensitive regions, under climate change, will challenge the sustained provision of fisheries productivity and other ecosystem services, especially at low latitudes (high confidence). By the mid-21st century, under $2^{\circ} \mathrm{C}$ global warming relative to pre-industrial temperatures, shifts in the geographical range of marine species will cause species richness and fisheries catch potential to increase, on average, at mid and high latitudes (high confidence) and to decrease at tropical latitudes and in semi-enclosed seas (Figure 2.6a) (medium confidence). The progressive expansion of Oxygen Minimum Zones and anoxic 'dead zones' in the oceans will further constrain fish habitats (medium confidence). Open-ocean net primary production is projected to redistribute and to decrease globally, by 2100 , under all RCP scenarios (medium confidence). Climate change adds to the threats of over-fishing and other non-climatic stressors (high confidence). \{WGII SPM B-2, 6.3-6.5, 7.4, 25.6, 28.3, 29.3, 30.6-30.7, Box CC-MB, Box CC-PP\}

Marine ecosystems, especially coral reefs and polar ecosystems, are at risk from ocean acidification (medium to high confidence). Ocean acidification has impacts on the physiology, behaviour and population dynamics of organisms. The impacts on individual species and the number of species affected in species groups increase from RCP4.5 to RCP8.5. Highly calcified molluscs, echinoderms and reef-building corals are more sensitive than crustaceans (high confidence) and fishes (low confidence) (Figure 2.6b). Ocean acidification acts together with other global changes (e.g., warming, progressively lower oxygen levels) and with local changes (e.g., pollution, eutrophication) (high confidence), leading to interactive, complex and amplified impacts for species and ecosystems (Figure 2.5b). \{WGII SPM B-2, Figure SPM.6B, 5.4, 6.3.2, 6.3.5, 22.3, 25.6, 28.3, 30.5, Figure 6-10, Box CC-CR, Box CC-OA, Box TS.7\}

Carbon stored in the terrestrial biosphere is susceptible to loss to the atmosphere as a result of climate change, deforestation and ecosystem degradation (high confidence). The aspects of climate change with direct effects on stored terrestrial carbon include high temperatures, drought and windstorms; indirect effects include increased risk of fires, pest and disease outbreaks. Increased tree mortality and associated forest dieback is projected to occur in many regions over the 21st century (medium confidence), posing risks for carbon storage, biodiversity, wood production, water quality, amenity and economic activity. There is a high risk of substantial carbon and methane emissions as a result of permafrost thawing. \{WGII SPM, 4.2-4.3, Figure 4-8, Box 4-2, Box 4-3, Box 4-4\}

Coastal systems and low-lying areas will increasingly experience submergence, flooding and erosion throughout the 21st century and beyond, due to sea level rise (very high confidence). The population and assets projected to be exposed to coastal risks as well as human pressures on coastal ecosystems will increase significantly in the coming decades due to population growth, economic development and urbanization (high confidence). Climatic and non-climatic drivers affecting coral reefs will erode habitats, increase coastline exposure to waves and storms and degrade environmental features important to fisheries and tourism (high confidence). Some low-lying developing countries and small island states are expected to face very high impacts that could have associated damage and adaptation costs of several percentage points of gross domestic product (GDP) (Figure 2.5c). \{WGII 5.3-5.5, 22.3, 24.4, 25.6, 26.3, 26.8, 29.4, Table 26-1, Box 25-1, Box CC-CR\}

\subsubsection{Water, food and urban systems, human health, security and livelihoods}

The fractions of the global population that will experience water scarcity and be affected by major river floods are projected to increase with the level of warming in the 21st century (robust evidence, high agreement). \{WGII 3.4-3.5, 26.3, 29.4, Table 3-2, Box 25-8\} 
(a)

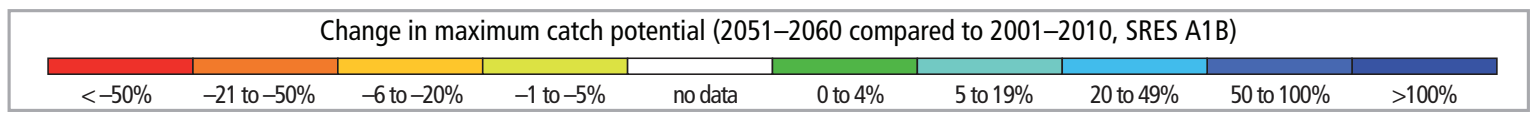

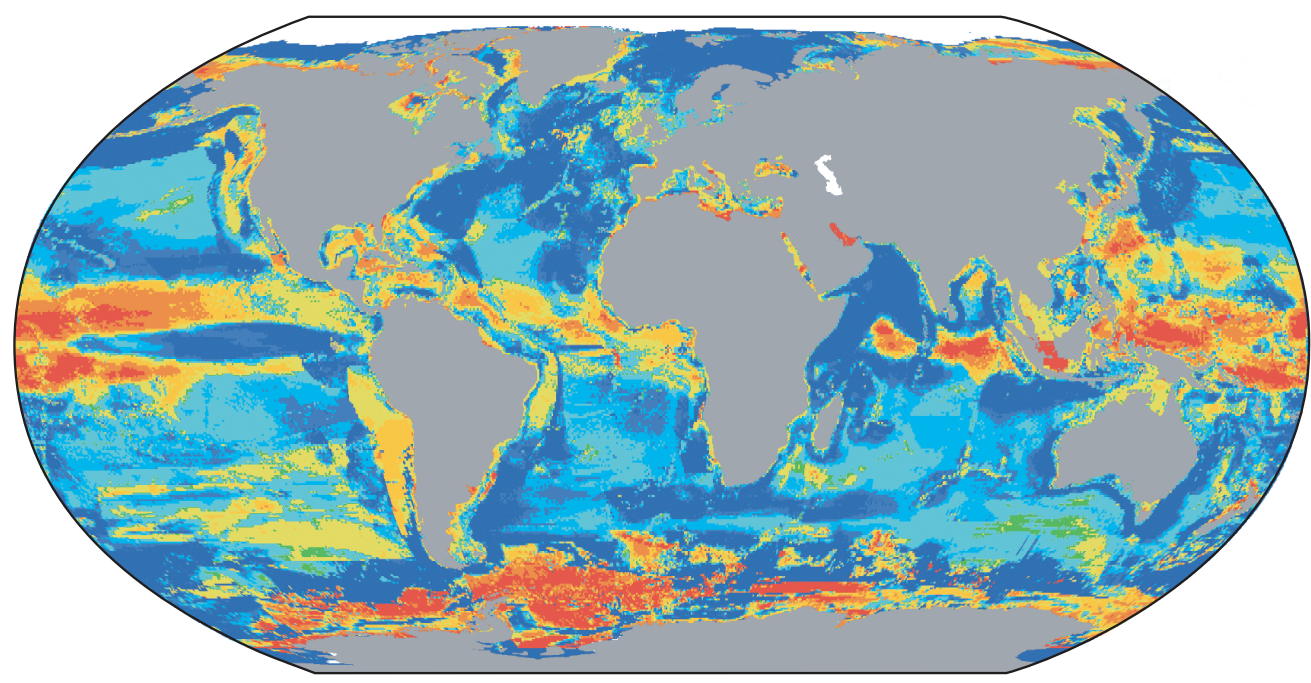

(b)
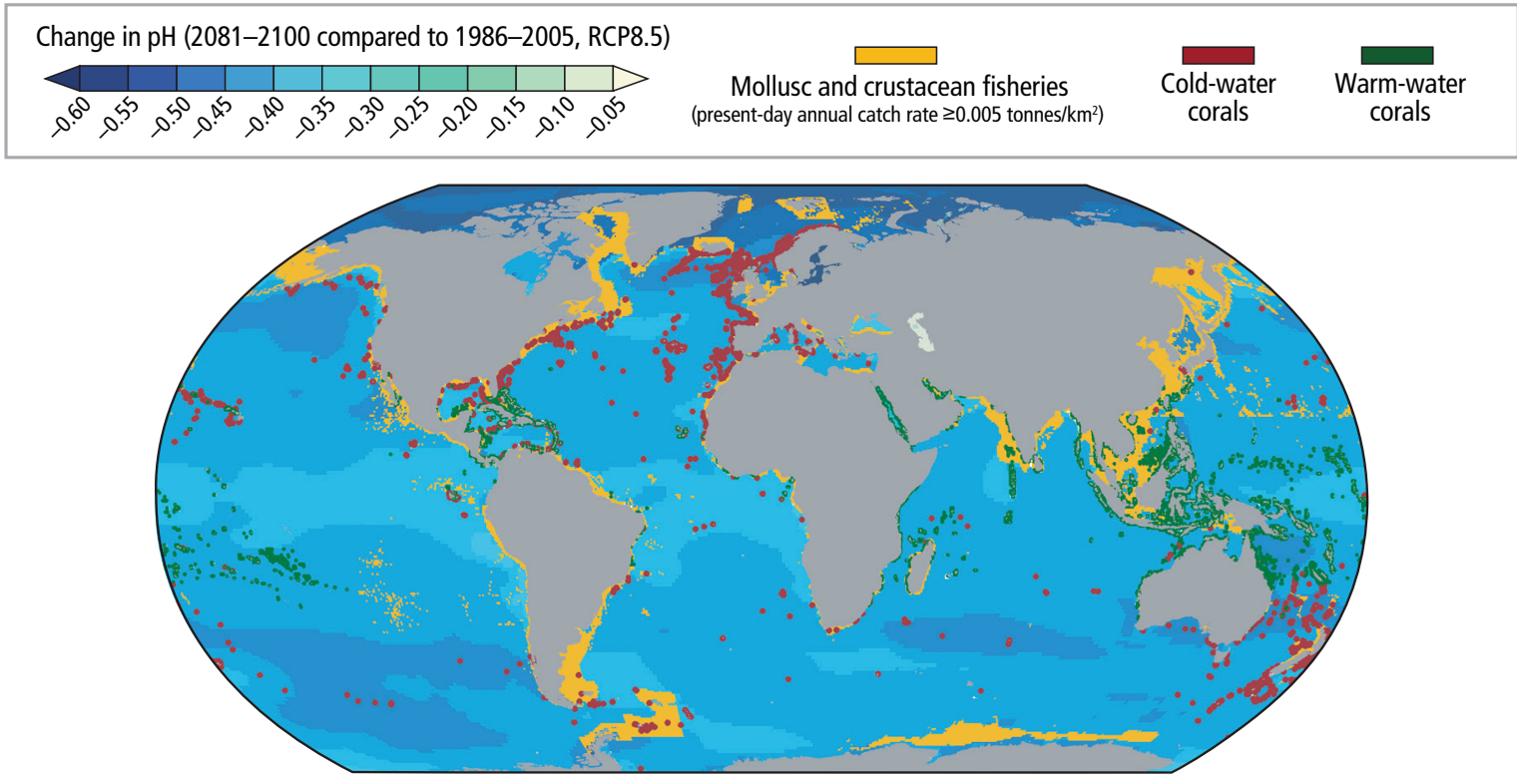

Molluscs

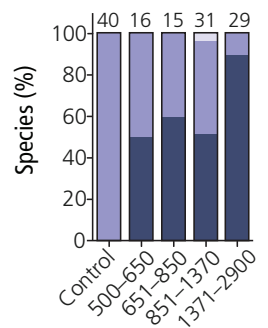

Crustaceans

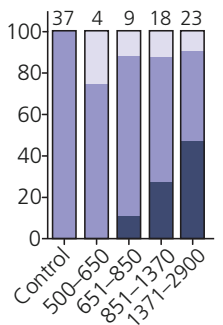

Cold-water corals

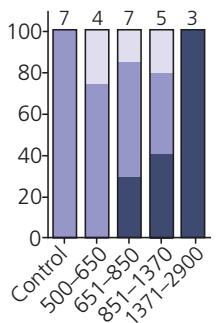

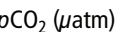

Figure 2.6 | Climate change risks for fisheries. (a) Projected global redistribution of maximum catch potential of $\sim 1000$ species of exploited fishes and invertebrates, comparing the 10-year averages over 2001-2010 and 2051-2060, using ocean conditions based on a single climate model under a moderate to high warming scenario $\left(2^{\circ} \mathrm{C}\right.$ warming relative to pre-industrial temperatures), without analysis of potential impacts of overfishing or ocean acidification. (b) Marine mollusc and crustacean fisheries (present-day estimated annual catch rates $\geq 0.005$ tonnes $/ \mathrm{km}^{2}$ ) and known locations of cold- and warm-water corals, depicted on a global map showing the projected distribution of surface ocean acidification by 2100 under RCP8.5. The bottom panel compares the percentage of species sensitive to ocean acidification for corals, molluscs and crustaceans, vulnerable animal phyla with socio-economic relevance (e.g., for coastal protection and fisheries). The number of species analysed across studies is given on top of the bars for each category of elevated $\mathrm{CO}_{2}$. For 2100, RCP scenarios falling within each $p \mathrm{CO}_{2}$ category are as follows: RCP4.5 for 500 to $650 \mu$ atm, RCP6.0 for 651 to $850 \mu$ atm and RCP8.5 for 851 to $1370 \mu$ atm. By 2150 , RCP8.5 falls within the 1371 to $2900 \mu$ atm category. The control category corresponds to $380 \mu$ atm (The unit $\mu$ atm is approximately equivalent to ppm in the atmosphere). [WGI Figure SPM.8, Box SPM.1, WGII SPM B-2, Figure SPM.6, 6.1, 6.3, 30.5, Figure 6-10, Figure 6-14\} 
Climate change over the 21st century is projected to reduce renewable surface water and groundwater resources in most dry subtropical regions (robust evidence, high agreement), intensifying competition for water among sectors (limited evidence, medium agreement). In presently dry regions, the frequency of droughts will likely increase by the end of the 21st century under RCP8.5 (medium confidence). In contrast, water resources are projected to increase at high latitudes (robust evidence, high agreement). The interaction of increased temperature; increased sediment, nutrient and pollutant loadings from heavy rainfall; increased concentrations of pollutants during droughts; and disruption of treatment facilities during floods will reduce raw water quality and pose risks to drinking water quality (medium evidence, high agreement). \{WGI 12.4, WGII 3.2, 3.4-3.6, 22.3, 23.9, 25.5, 26.3, Table 3-2, Table23-3, Box25-2, Box CC-RF, Box CC-WE\}

All aspects of food security are potentially affected by climate change, including food production, access, use and price stability (high confidence). For wheat, rice and maize in tropical and temperate regions, climate change without adaptation is projected to negatively impact production at local temperature increases of $2^{\circ} \mathrm{C}$ or more above late 20th century levels, although individual locations may benefit (medium confidence). Projected impacts vary across crops and regions and adaptation scenarios, with about $10 \%$ of projections for the 2030-2049 period showing yield gains of more than $10 \%$, and about $10 \%$ of projections showing yield losses of more than $25 \%$, compared with the late 20th century. Global temperature increases of $\sim 4^{\circ} \mathrm{C}$ or more above late 20th century levels, combined with increasing food demand, would pose large risks to food security, both globally and regionally (high confidence) (Figure 2.4, 2.7). The relationship between global and regional warming is explained in 2.2.1. \{WGII 6.3-6.5, 7.4-7.5, 9.3, 22.3, 24.4, 25.7, 26.5, Table 7-2, Table 7-3, Figure 7-1, Figure 7-4, Figure 7-5, Figure 7-6, Figure 7-7, Figure 7-8, Box 7-1\}

Until mid-century, projected climate change will impact human health mainly by exacerbating health problems that already exist (very high confidence). Throughout the 21st century, climate change is expected to lead to increases in ill-health in many regions and especially in developing countries with low income, as compared to a baseline without climate change (high confidence). Health impacts include greater likelihood of injury and death due to more intense heat waves and fires, increased risks from foodborne and waterborne diseases and loss of work capacity and reduced labour productivity in vulnerable populations (high confidence). Risks of undernutrition in poor regions will increase (high confidence). Risks from vector-borne diseases are projected to generally increase with warming, due to the extension of the infection area and season, despite reductions in some areas that become too hot for disease vectors (medium confidence). Globally, the magnitude and severity of negative impacts will increasingly outweigh positive impacts (high confidence). By 2100 for RCP8.5, the combination of high temperature and humidity in some areas for parts of the year is expected to compromise common human activities, including growing food and working outdoors (high confidence). \{WGII SPM B-2, 8.2, 11.3-11.8, 19.3, 22.3, 25.8, 26.6, Figure 25-5, Box CC-HS\}

In urban areas, climate change is projected to increase risks for people, assets, economies and ecosystems, including risks from heat stress, storms and extreme precipitation, inland and coastal flooding, landslides, air pollution, drought, water scarcity, sea level rise and storm surges (very high confidence). These risks will be amplified for those lacking essential infrastructure and services or living in exposed areas. \{WGII 3.5, 8.2-8.4, 22.3, 24.4-24.5, 26.8, Table 8-2, Box 25-9, Box CC-HS\}

Rural areas are expected to experience major impacts on water availability and supply, food security, infrastructure and agricultural incomes, including shifts in the production areas of food and non-food crops around the world (high confidence). These impacts will disproportionately affect the welfare of the poor in rural areas, such as female-headed households and those with limited access to land, modern agricultural inputs, infrastructure and education. \{WGII 5.4, 9.3,25.9, 26.8, 28.2, 28.4, Box 25-5\}

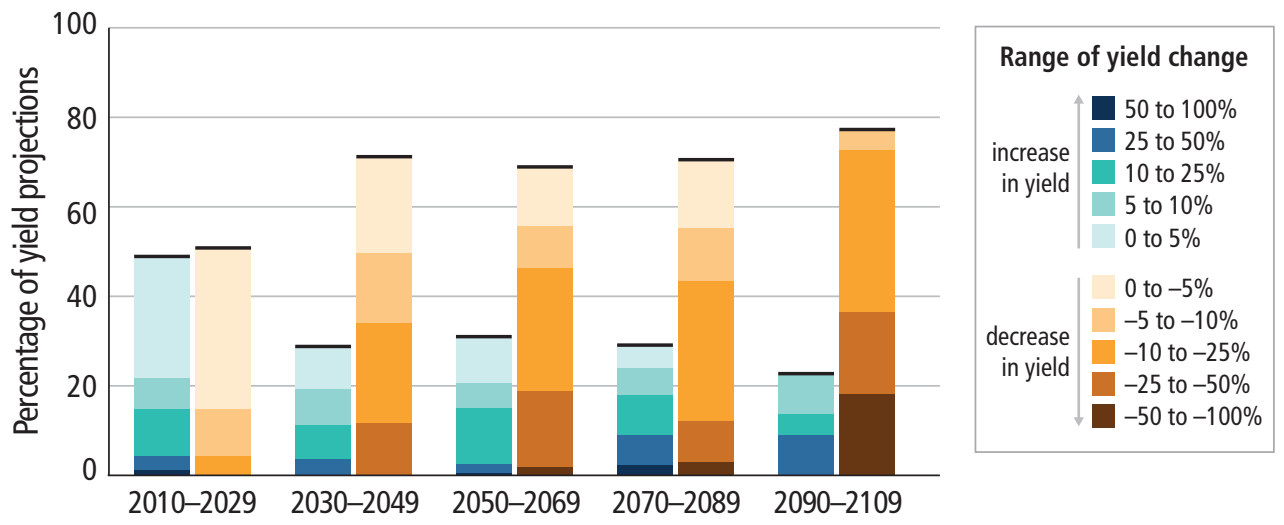

Figure 2.7| Summary of projected changes in crop yields (mostly wheat, maize, rice and soy) due to climate change over the 21st century. The figure combines 1090 data points from crop model projections, covering different emission scenarios, tropical and temperate regions and adaptation and no-adaptation cases. The projections are sorted into the 20-year periods (horizontal axis) during which their midpoint occurs. Changes in crop yields are relative to late 20th century levels and data for each time period sum to $100 \%$. Relatively few studies have considered impacts on cropping systems for scenarios where global mean temperatures increase by $4^{\circ} \mathrm{C}$ or more. \{WGII Figure SPM.7\} 
Table 2.3 | Examples of global key risks for different sectors, including the potential for risk reduction through adaptation and mitigation, as well as limits to adaptation. Each key risk is assessed as very low, low, medium, high or very high. Risk levels are presented for three time frames: present, near term (here, for 2030-2040) and long term (here, for 2080-2100). In the near term, projected levels of global mean temperature increase do not diverge substantially across different emission scenarios. For the long term, risk levels are presented for two possible futures $\left(2^{\circ} \mathrm{C}\right.$ and $4^{\circ} \mathrm{C}$ global mean temperature increase above pre-industrial levels). For each time frame, risk levels are indicated for a continuation of current adaptation and assuming high levels of current or future adaptation. Risk levels are not necessarily comparable, especially across regions. Relevant climate variables are indicated by icons. \{WGII Table TS.4\}

\begin{tabular}{|c|c|c|c|c|c|c|c|c|c|c|}
\hline \multicolumn{9}{|c|}{ Climate-related drivers of impacts } & \multirow{2}{*}{\multicolumn{2}{|c|}{$\begin{array}{c}\text { Level of risk \& potential for adaptation } \\
\text { Potential for additional adaptation } \\
\vdots \text { to reduce risk } \\
\end{array}$}} \\
\hline & & & & & & & & & & \\
\hline $\begin{array}{l}\text { Warming } \\
\text { trend }\end{array}$ & $\begin{array}{l}\text { Extreme } \\
\text { temperature }\end{array}$ & $\begin{array}{l}\text { Drying } \\
\text { trend }\end{array}$ & $\begin{array}{l}\text { Extreme } \\
\text { precipitation }\end{array}$ & $\begin{array}{l}\text { Damaging } \\
\text { cyclone }\end{array}$ & Flooding & $\begin{array}{l}\text { Storm } \\
\text { surge }\end{array}$ & $\begin{array}{c}\text { Ocean } \\
\text { acidification }\end{array}$ & $\begin{array}{l}\text { Carbon dioxide } \\
\text { fertilisation }\end{array}$ & $\begin{array}{l}\text { Risk level with } \\
\text { high adaptation }\end{array}$ & $\begin{array}{l}\text { Risk level with } \\
\text { current adaptation }\end{array}$ \\
\hline
\end{tabular}

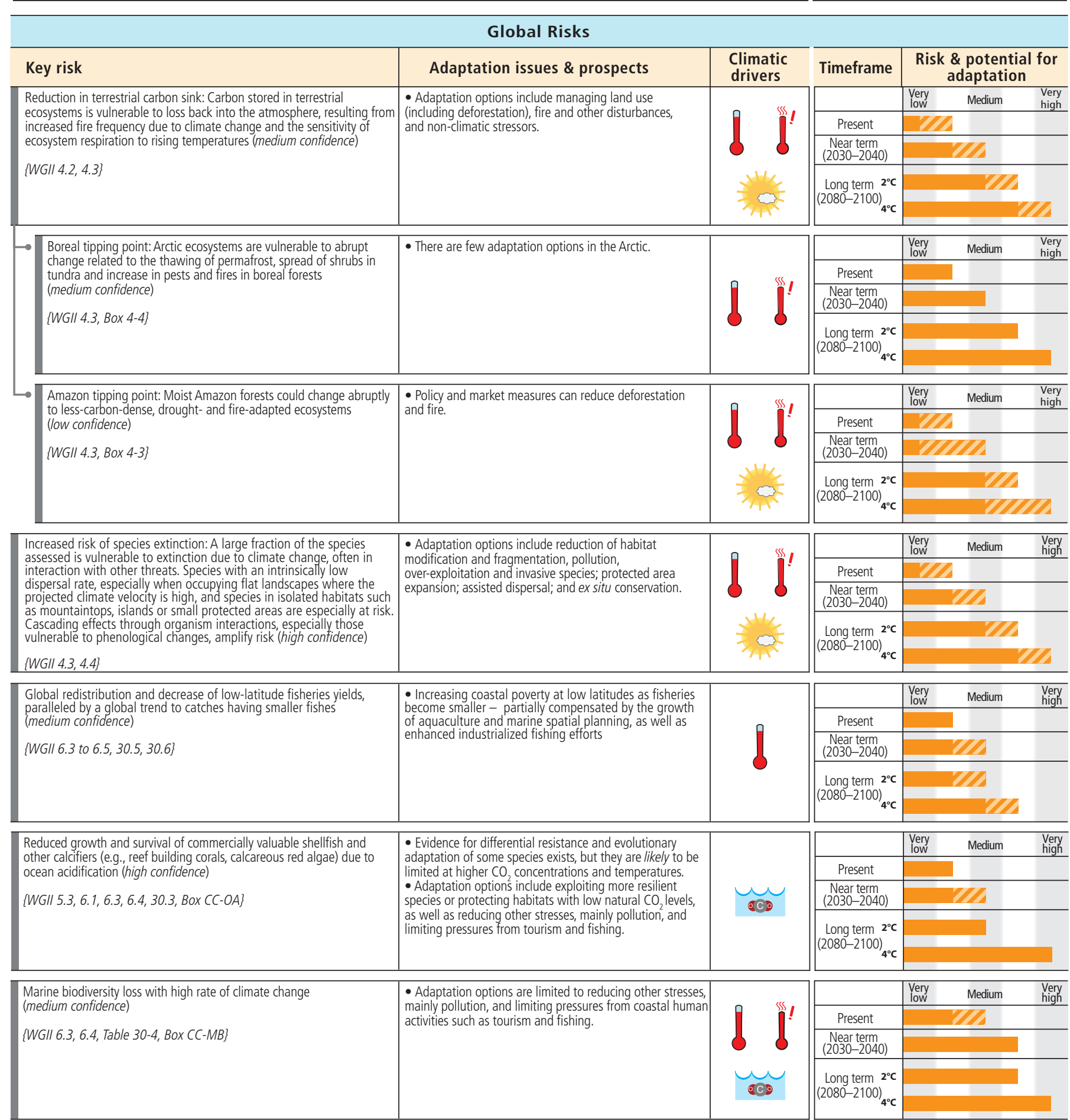


Table 2.3 (continued)

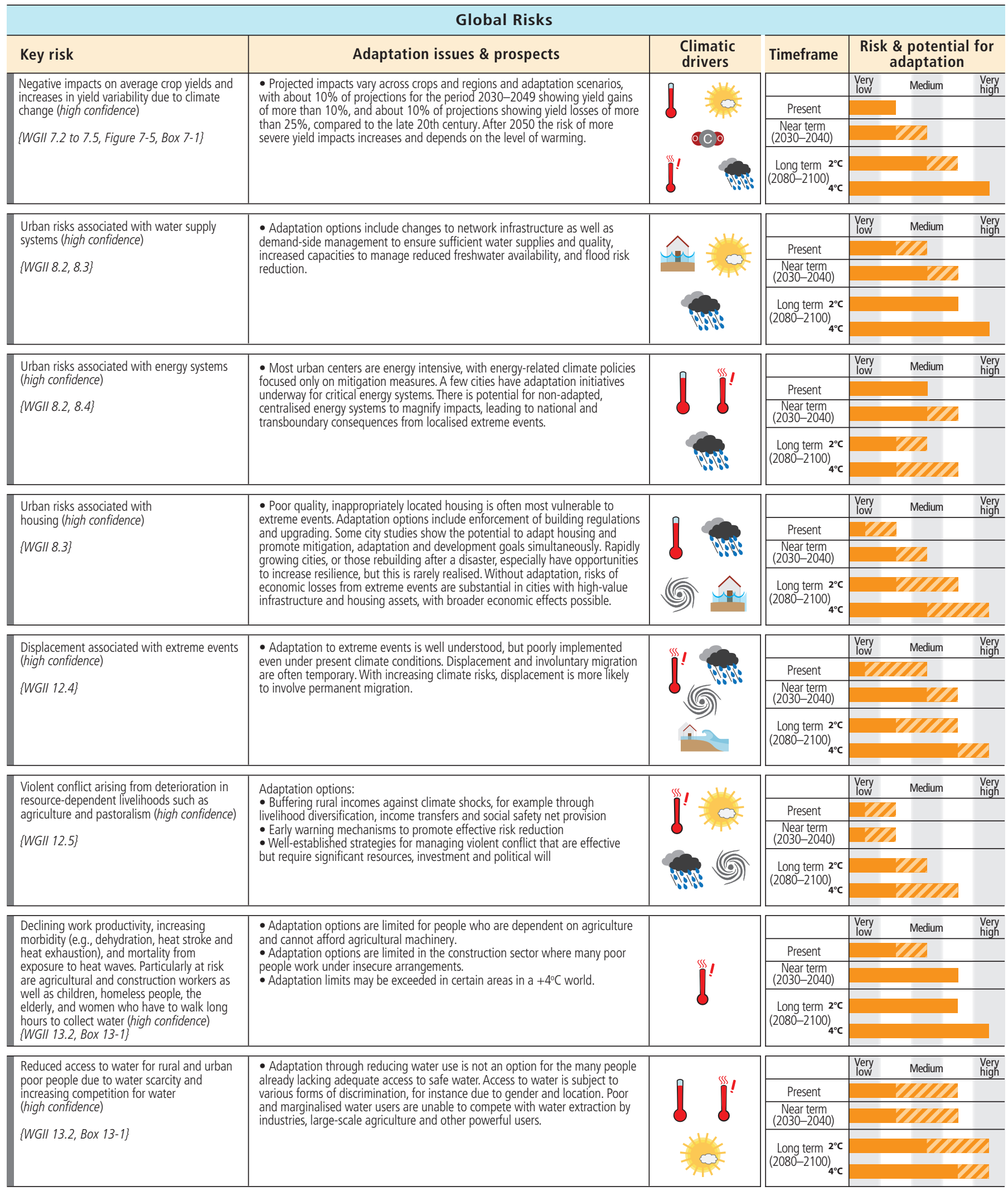




\section{Box 2.4 | Reasons For Concern Regarding Climate Change}

Five Reasons For Concern (RFCs) have provided a framework for summarizing key risks since the IPCC Third Assessment Report. They illustrate the implications of warming and of adaptation limits for people, economies and ecosystems across sectors and regions. They provide one starting point for evaluating dangerous anthropogenic interference with the climate system. All warming levels in the text of Box 2.4 are relative to the $1986-2005$ period. Adding $\sim 0.6^{\circ} \mathrm{C}$ to these warming levels roughly gives warming relative to the 1850-1900 period, used here as a proxy for pre-industrial times (right-hand scale in Box 2.4, Figure 1). \{WGII Assessment Box SPM.1\}

The five RFCs are associated with:

1. Unique and threatened systems: Some ecosystems and cultures are already at risk from climate change (high confidence). With additional warming of around $1{ }^{\circ} \mathrm{C}$, the number of unique and threatened systems at risk of severe consequences increases. Many systems with limited adaptive capacity, particularly those associated with Arctic sea ice and coral reefs, are subject to very high risks with additional warming of $2^{\circ} \mathrm{C}$. In addition to risks resulting from the magnitude of warming, terrestrial species are also sensitive to the rate of warming, marine species to the rate and degree of ocean acidification and coastal systems to sea level rise (Figure 2.5).

2. Extreme weather events: Climate change related risks from extreme events, such as heat waves, heavy precipitation and coastal flooding, are already moderate (high confidence). With $1^{\circ} \mathrm{C}$ additional warming, risks are high (medium confidence). Risks associated with some types of extreme events (e.g., extreme heat) increase progressively with further warming (high confidence).

3. Distribution of impacts: Risks are unevenly distributed between groups of people and between regions; risks are generally greater for disadvantaged people and communities everywhere. Risks are already moderate because of regional differences in observed climate change impacts, particularly for crop production (medium to high confidence). Based on projected decreases in regional crop yields and water availability, risks of unevenly distributed impacts are high under additional warming of above $2^{\circ} \mathrm{C}$ (medium confidence).

4. Global aggregate impacts: Risks of global aggregate impacts are moderate under additional warming of between $1{ }^{\circ} \mathrm{C}$ and $2^{\circ} \mathrm{C}$, reflecting impacts on both the Earth's biodiversity and the overall global economy (medium confidence). Extensive biodiversity loss, with associated loss of ecosystem goods and services, leads to high risks at around $3^{\circ} \mathrm{C}$ additional warming (high confidence). Aggregate economic damages accelerate with increasing temperature (limited evidence, high agreement), but few quantitative estimates are available for additional warming of above $3^{\circ} \mathrm{C}$.

5. Large-scale singular events: With increasing warming, some physical and ecological systems are at risk of abrupt and/or irreversible changes (see Section 2.4). Risks associated with such tipping points are moderate between 0 and $1^{\circ} \mathrm{C}$ additional warming, since there are signs that both warm-water coral reefs and Arctic ecosystems are already experiencing irreversible regime shifts (medium confidence). Risks increase at a steepening rate under an additional warming of 1 to $2^{\circ} \mathrm{C}$ and become high above $3^{\circ} \mathrm{C}$, due to the potential for large and irreversible sea level rise from ice sheet loss. For sustained warming above some threshold greater than $\sim 0.5^{\circ} \mathrm{C}$ additional warming (low confidence) but less than $\sim 3.5^{\circ} \mathrm{C}$ (medium confidence), near-complete loss of the Greenland ice sheet would occur over a millennium or more, eventually contributing up to $7 \mathrm{~m}$ to global mean sea level rise. 
Box 2.4 (continued)

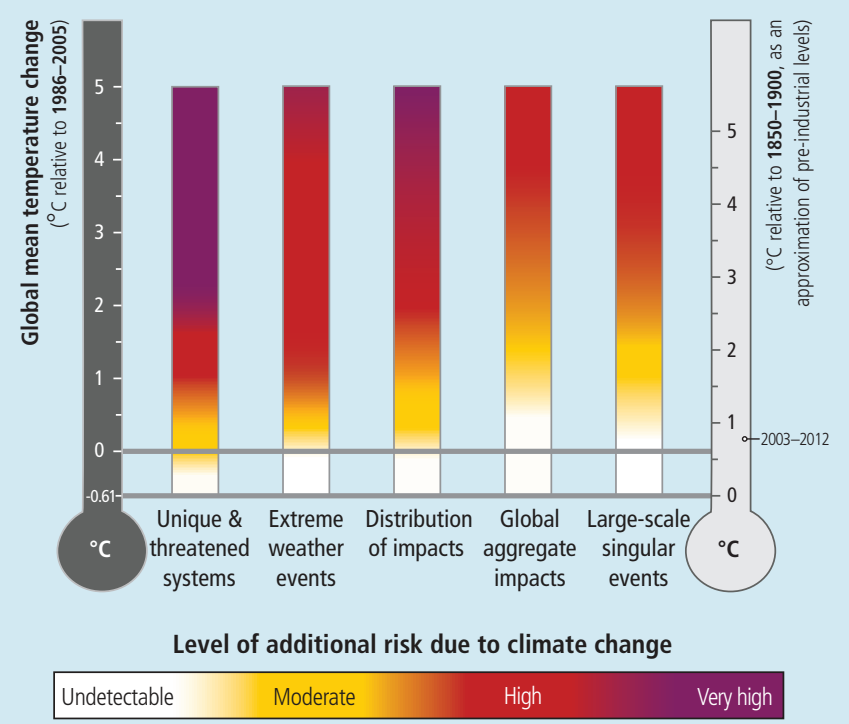

Box 2.4, Figure 1 | Risks associated with Reasons For Concern at a global scale are shown for increasing levels of climate change. The colour shading indicates the additional risk due to climate change when a temperature level is reached and then sustained or exceeded. White indicates no associated impacts are detectable and attributable to climate change. Yellow indicates that associated impacts are both detectable and attributable to climate change with at least medium confidence. Red indicates severe and widespread impacts. Purple, introduced in this assessment, shows that very high risk is indicated by all key risk criteria. \{WGII Assessment Box SPM. 1, Figure 19-4\}

Aggregate economic losses accelerate with increasing temperature (limited evidence, high agreement), but global economic impacts from climate change are currently difficult to estimate. With recognized limitations, the existing incomplete estimates of global annual economic losses for warming of $2.5^{\circ} \mathrm{C}$ above pre-industrial levels are 0.2 to $2.0 \%$ of income (medium evidence, medium agreement). Changes in population, age structure, income, technology, relative prices, lifestyle, regulation and governance are projected to have relatively larger impacts than climate change, for most economic sectors (medium evidence, high agreement). More severe and/or frequent weather hazards are projected to increase disaster-related losses and loss variability, posing challenges for affordable insurance, particularly in developing countries. International dimensions such as trade and relations among states are also important for understanding the risks of climate change at regional scales. (Box 3.1) \{WGII 3.5, 10.2, 10.7, 10.9-10.10, 17.4-17.5, 25.7, 26.7-26.9, Box 25-7\}

From a poverty perspective, climate change impacts are projected to slow down economic growth, make poverty reduction more difficult, further erode food security and prolong existing poverty traps and create new ones, the latter particularly in urban areas and emerging hotspots of hunger (medium confidence). Climate change impacts are expected to exacerbate poverty in most developing countries and create new poverty pockets in countries with increasing inequality, in both developed and developing countries (Figure 2.4). \{WGII 8.1, 8.3-8.4, 9.3, 10.9, 13.2-13.4, 22.3, 26.8\}

Climate change is projected to increase displacement of people (medium evidence, high agreement). Displacement risk increases when populations that lack the resources for planned migration experience higher exposure to extreme weather events, such as floods and droughts. Expanding opportunities for mobility can reduce vulnerability for such populations. Changes in migration patterns can be responses to both extreme weather events and longer term climate variability and change, and migration can also be an effective adaptation strategy. \{WGII 9.3, 12.4, 19.4, 22.3, 25.9\}

Climate change can indirectly increase risks of violent conflict by amplifying well-documented drivers of these conflicts, such as poverty and economic shocks (medium confidence). Multiple lines of evidence relate climate variability to some forms of conflict. $\{W G I I S P M, 12.5,13.2,19.4\}$

\subsection{Climate change beyond 2100, irreversibility and abrupt changes}

Many aspects of climate change and its associated impacts will continue for centuries, even if anthropogenic emissions of greenhouse gases are stopped. The risks of abrupt or irreversible changes increase as the magnitude of the warming increases.

Warming will continue beyond 2100 under all RCP scenarios except RCP2.6. Surface temperatures will remain approximately constant at elevated levels for many centuries after a complete cessation of net anthropogenic $\mathrm{CO}_{2}$ emissions (see Section 2.2.5 for the relationship between $\mathrm{CO}_{2}$ emissions and global temperature change.). A large fraction of anthropogenic climate change resulting from $\mathrm{CO}_{2}$ emissions is irreversible on a multi-century to millennial timescale, except in the 


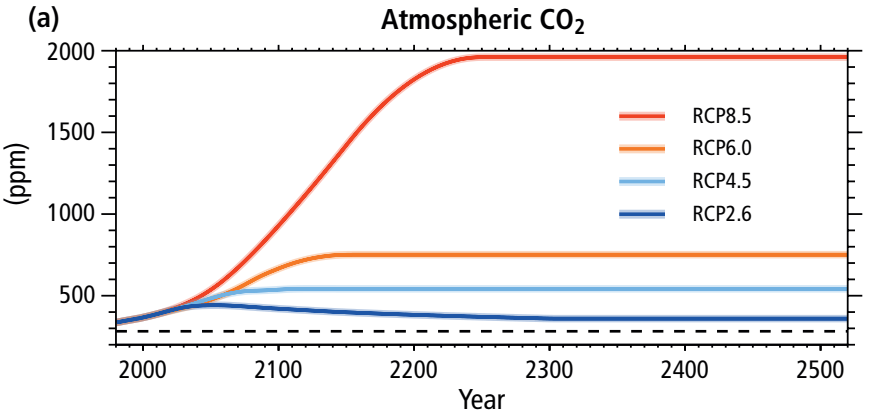

(b) Surface temperature change

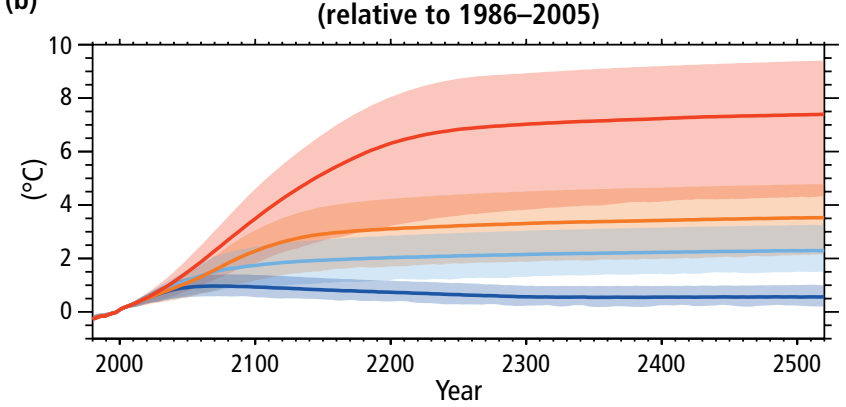

(c)

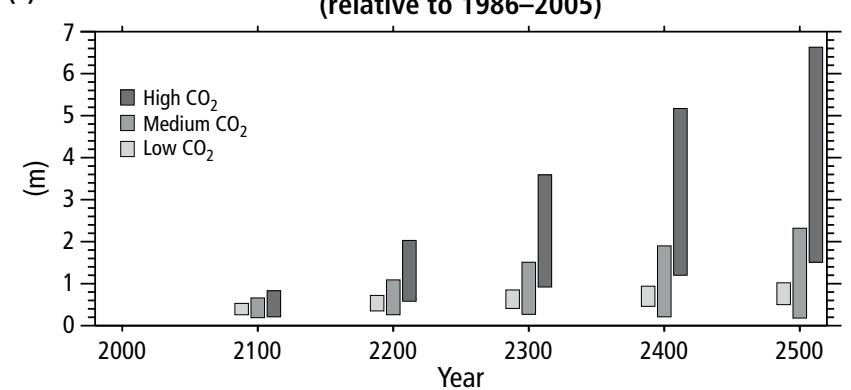

Figure 2.8 (a) Atmospheric carbon dioxide $\left(\mathrm{CO}_{2}\right)$ and (b) projected global mean surface temperature change as simulated by Earth System Models of Intermediate Complexity (EMICs) for the four Representative Concentration Pathways (RCPs) up to 2300 (relative to 1986-2005) followed by a constant (year 2300 level) radiative forcing. A 10-year smoothing was applied. The dashed line on (a) indicates the pre-industrial $\mathrm{CO}_{2}$ concentration. (c) Sea level change projections grouped into three categories according to the concentration of greenhouse gas (in $\mathrm{CO}_{2}$-eq) in 2100 (low: concentrations that peak and decline and remain below 500 ppm, as in scenario RCP2.6; medium: 500 to 700 ppm, including RCP4.5; high: concentrations that are above 700 ppm but below 1500 ppm, as in scenario RCP6.0 and RCP8.5). The bars in (c) show the maximum possible spread that can be obtained with the few available model results (and should not be interpreted as uncertainty ranges). These models likely underestimate the Antarctica ice sheet contribution, resulting in an underestimation of projected sea level rise beyond 2100. \{WGI Figure 12.43, Figure 13.13, Table 13.8, WGII SPM B-2\}

case of a large net removal of $\mathrm{CO}_{2}$ from the atmosphere over a sustained period (Figure 2.8a, b). \{WGI SPM E.1, SPM E.8, 12.5.2\}

Stabilization of global average surface temperature does not imply stabilization for all aspects of the climate system. Shifting biomes, re-equilibrating soil carbon, ice sheets, ocean temperatures and associated sea level rise all have their own intrinsic long timescales that will result in ongoing changes for hundreds to thousands of years after global surface temperature has been stabilized. \{WGI SPM E.8, 12.5.2-12.5.4, WGII 4.2\}

Ocean acidification will continue for centuries if $\mathrm{CO}_{2}$ emissions continue, it will strongly affect marine ecosystems (high confidence), and the impact will be exacerbated by rising temperature extremes (Figure 2.5b). \{WGI 3.8.2, 6.4.4, WGII SPM B-2, 6.3.2, 6.3.5, 30.5, Box CC-OA\}

Global mean sea level rise will continue for many centuries beyond 2100 (virtually certain). The few available analyses that go beyond 2100 indicate sea level rise to be less than $1 \mathrm{~m}$ above the pre-industrial level by 2300 for GHG concentrations that peak and decline and remain below $500 \mathrm{ppm} \mathrm{CO}$-eq, as in scenario RCP2.6. For a radiative forcing that corresponds to a $\mathrm{CO}_{2}$-eq concentration in 2100 that is above $700 \mathrm{ppm}$ but below $1500 \mathrm{ppm}$, as in scenario RCP8.5, the projected rise is $1 \mathrm{~m}$ to more than $3 \mathrm{~m}$ by 2300 (medium confidence) (Figure 2.8c). There is low confidence in the available models' ability to project solid ice discharge from the Antarctic ice sheet. Hence, these models likely underestimate the Antarctica ice sheet contribution, resulting in an underestimation of projected sea level rise beyond 2100. \{WGI SPM E.8, 13.4.4, 13.5.4\}

There is little evidence in global climate models of a tipping point or critical threshold in the transition from a perennially ice-covered to a seasonally ice-free Arctic Ocean, beyond which further sea-ice loss is unstoppable and irreversible. \{WGI 12.5.5\}

There is low confidence in assessing the evolution of the Atlantic Meridional Overturning Circulation beyond the 21st century because of the limited number of analyses and equivocal results. However, a collapse beyond the 21st century for large sustained warming cannot be excluded. \{WGI SPM E.4, 12.4.7, 12.5.5\}

Sustained mass loss by ice sheets would cause larger sea level rise, and part of the mass loss might be irreversible. There is high confidence that sustained global mean warming greater than a threshold would lead to the near-complete loss of the Greenland ice sheet over a millennium or more, causing a sea level rise of up to $7 \mathrm{~m}$. Current estimates indicate that the threshold is greater than about $1^{\circ} \mathrm{C}$ (low confidence) but less than about $4^{\circ} \mathrm{C}$ (medium confidence) of global warming with respect to pre-industrial temperatures. Abrupt and irreversible ice loss from a potential instability of marine-based sectors of the Antarctic ice sheet in response to climate forcing is possible, but current evidence and understanding is insufficient to make a quantitative assessment. \{WGI SPM E.8, 5.6.2, 5.8.1, 13.4.3, 13.5.4\}

Within the 21st century, magnitudes and rates of climate change associated with medium to high emission scenarios (RCP4.5, RCP6.0 and RCP8.5) pose a high risk of abrupt and irreversible regional-scale change in the composition, structure and function of marine, terrestrial and freshwater ecosystems, including wetlands (medium confidence), as well as warm water coral reefs (high confidence). Examples that could substantially amplify climate change are the boreal-tundra Arctic system (medium confidence) and the Amazon forest (Iow confidence). \{WGIl 4.3.3.1, Box 4.3, Box 4.4, 5.4.2.4, 6.3.1-6.3.4, 6.4.2, 30.5.3-30.5.6, Box CC-CR, Box CC-MB\}

A reduction in permafrost extent is virtually certain with continued rise in global temperatures. Current permafrost areas are projected to become a net emitter of carbon $\left(\mathrm{CO}_{2}\right.$ and $\left.\mathrm{CH}_{4}\right)$ with a loss of 180 to $920 \mathrm{GtCO}_{2}$ (50 to $250 \mathrm{GtC}$ ) under RCP8.5 over the 21 st century (Iow confidence). \{WGI TFE.5, 6.4.3.4, 12.5.5, WGII 4.3.3.4\} 
Future Pathways for Adaptation, Mitigation and Sustainable Development 


\section{Topic 3: Future Pathways for Adaption, Mitigation and Sustainable Development}

Adaptation and mitigation are complementary strategies for reducing and managing the risks of climate change. Substantial emissions reductions over the next few decades can reduce climate risks in the 21st century and beyond, increase prospects for effective adaptation, reduce the costs and challenges of mitigation in the longer term and contribute to climate-resilient pathways for sustainable development.

Adaptation and mitigation are two complementary strategies for responding to climate change. Adaptation is the process of adjustment to actual or expected climate and its effects in order to either lessen or avoid harm or exploit beneficial opportunities. Mitigation is the process of reducing emissions or enhancing sinks of greenhouse gases (GHGs), so as to limit future climate change. Both adaptation and mitigation can reduce and manage the risks of climate change impacts. Yet adaptation and mitigation can also create other risks, as well as benefits. Strategic responses to climate change involve consideration of climate-related risks along with the risks and co-benefits of adaptation and mitigation actions. [WGII SPM A-3, SPM C, Glossary, WGIII SPM.2, 4.1, 5.1, Glossary\}

Mitigation, adaptation and climate impacts can all result in transformations to and changes in systems. Depending on the rate and magnitude of change and the vulnerability and exposure of human and natural systems, climate change will alter ecosystems, food systems, infrastructure, coastal, urban and rural areas, human health and livelihoods. Adaptive responses to a changing climate require actions that range from incremental changes to more fundamental, transformational changes ${ }^{34}$. Mitigation can involve fundamental changes in the way that human societies produce and use energy services and land. \{WGII B, C, TS C, Box TS.8, Glossary, WGIII SPM.4\}

Topic 3 of this report examines the factors that influence the assessment of mitigation and adaptation strategies. It considers the benefits, risks, incremental changes and potential transformations from different combinations of mitigation, adaptation and residual climate-related impacts. It considers how responses in the coming decades will influence options for limiting long-term climate change and opportunities for adapting to it. Finally, it considers factors-including uncertainty, ethical considerations and links to other societal goals- that may influence choices about mitigation and adaptation. Topic 4 then assesses the prospects for mitigation and adaptation on the basis of current knowledge of tools, options and policies.

\subsection{Foundations of decision-making about climate change}

Effective decision-making to limit climate change and its effects can be informed by a wide range of analytical approaches for evaluating expected risks and benefits, recognizing the importance of governance, ethical dimensions, equity, value judgments, economic assessments and diverse perceptions and responses to risk and uncertainty.

Sustainable development and equity provide a basis for assessing climate policies. Limiting the effects of climate change is necessary to achieve sustainable development and equity, including poverty eradication. Countries' past and future contributions to the accumulation of GHGs in the atmosphere are different, and countries also face varying challenges and circumstances and have different capacities to address mitigation and adaptation. Mitigation and adaptation raise issues of equity, justice and fairness and are necessary to achieve sustainable development and poverty eradication. Many of those most vulnerable to climate change have contributed and contribute little to GHG emissions. Delaying mitigation shifts burdens from the present to the future, and insufficient adaptation responses to emerging impacts are already eroding the basis for sustainable development. Both adaptation and mitigation can have distributional effects locally, nationally and internationally, depending on who pays and who benefits. The process of decision-making about climate change, and the degree to which it respects the rights and views of all those affected, is also a concern of justice. \{WGII 2.2, 2.3, 13.3, 13.4, 17.3, 20.2, 20.5, WGIII SPM.2, 3.3, 3.10, 4.1.2, 4.2, 4.3, 4.5, $4.6,4.8\}$

Effective mitigation will not be achieved if individual agents advance their own interests independently. Climate change has the characteristics of a collective action problem at the global scale, because most GHGs accumulate over time and mix globally, and emissions by any agent (e.g., individual, community, company, country) affect other agents. Cooperative responses, including international cooperation, are therefore required to effectively mitigate GHG emissions and address other climate change issues. The effectiveness of adaptation can be enhanced through complementary actions across levels, including international cooperation. The evidence suggests that outcomes seen as equitable can lead to more effective cooperation. \{WGII 20.3.1, WGIII SPM.2, TS.1, 1.2, 2.6, 3.2, 4.2, 13.2, 13.3\}

Decision-making about climate change involves valuation and mediation among diverse values and may be aided by the analytic methods of several normative disciplines. Ethics analyses the different values involved and the relations between them. Recent political philosophy has investigated the question of responsibility for the effects of emissions. Economics and decision analysis provide

34 Transformation is used in this report to refer to a change in the fundamental attributes of a system (see Glossary). Transformations can occur at multiple levels; at the national level, transformation is considered most effective when it reflects a country's own visions and approaches to achieving sustainable development in accordance with its national circumstances and priorities. \{WGII SPM C-2, 2-13, 20.5, WGIII SPM, 6-12\} 
quantitative methods of valuation which can be used for estimating the social cost of carbon (see Box 3.1), in cost-benefit and costeffectiveness analyses, for optimization in integrated models and elsewhere. Economic methods can reflect ethical principles, and take account of non-marketed goods, equity, behavioural biases, ancillary benefits and costs and the differing values of money to different people. They are, however, subject to well-documented limitations. \{WGII 2.2, 2.3, WGIII SPM.2, Box TS.2, 2.4, 2.5, 2.6, 3.2-3.6, 3.9.4\}

Analytical methods of valuation cannot identify a single best balance between mitigation, adaptation and residual climate impacts. Important reasons for this are that climate change involves extremely complex natural and social processes, there is extensive disagreement about the values concerned, and climate change impacts and mitigation approaches have important distributional effects. Nevertheless, information on the consequences of emissions pathways to alternative climate goals and risk levels can be a useful input into decision-making processes. Evaluating responses to climate change involves assessment of the widest possible range of impacts, including low-probability outcomes with large consequences. \{WGII 1.1.4, 2.3, 2.4, 17.3, 19.6, 19.7, WGIII 2.5, 2.6, 3.4, 3.7, Box 3-9\}

Effective decision-making and risk management in the complex environment of climate change may be iterative: strategies can often be adjusted as new information and understanding develops during implementation. However, adaptation and mitigation choices in the near term will affect the risks of climate change throughout the 21st century and beyond, and prospects for climate-resilient pathways for sustainable development depend on what is achieved through mitigation. Opportunities to take advantage of positive synergies between adaptation and mitigation may decrease with time, particularly if mitigation is delayed too long. Decision-making about climate change is influenced by how individuals and organizations perceive risks and uncertainties and take them into account. They sometimes use simplified decision rules, overestimate or underestimate risks and are biased towards the status quo. They differ in their degree of risk aversion and the relative importance placed on near-term versus long-term ramifications of specific actions. Formalized analytical methods for decision-making under uncertainty can account accurately for risk, and focus attention on both short- and long-term consequences. \{WGII SPM A-3, SPM C-2, 2.1-2.4, 3.6, 14.1-14.3, 15.2-15.4, 17.117.3, 17.5, 20.2, 20.3, 20.6, WGIII SPM.2, 2.4, 2.5, 5.5, 16.4\}

\subsection{Climate change risks reduced by adaptation and mitigation}

Without additional mitigation efforts beyond those in place today, and even with adaptation, warming by the end of the 21st century will lead to high to very high risk of severe, widespread and irreversible impacts globally (high confidence). Mitigation involves some level of co-benefits and of risks due to adverse side effects, but these risks do not involve the same possibility of severe, widespread and irreversible impacts as risks from climate change, increasing the benefits from near-term mitigation efforts.
The risks of climate change, adaptation and mitigation differ in nature, timescale, magnitude and persistence (high confidence). Risks from adaptation include maladaptation and negative ancillary impacts. Risks from mitigation include possible adverse side effects of large-scale deployment of low-carbon technology options and economic costs. Climate change risks may persist for millennia and can involve very high risk of severe impacts and the presence of significant irreversibilities combined with limited adaptive capacity. In contrast, the stringency of climate policies can be adjusted much more quickly in response to observed consequences and costs and create lower risks of irreversible consequences $(3.3,3.4,4.3)$. \{WGI SPM E.8, 12.4, 12.5.2, 13.5, WGII 4.2, 17.2, 19.6, WGIII TS.3.1.4, Table TS.4, Table TS.5, Table TS.6, Table TS.7, Table TS.8, 2.5, 6.6\}

Mitigation and adaptation are complementary approaches for reducing risks of climate change impacts. They interact with one another and reduce risks over different timescales (high confidence). Benefits from adaptation can already be realized in addressing current risks and can be realized in the future for addressing emerging risks. Adaptation has the potential to reduce climate change impacts over the next few decades, while mitigation has relatively little influence on climate outcomes over this timescale. Near-term and longerterm mitigation and adaptation, as well as development pathways, will determine the risks of climate change beyond mid-century. The potential for adaptation differs across sectors and will be limited by institutional and capacity constraints, increasing the long-term benefits of mitigation (high confidence). The level of mitigation will influence the rate and magnitude of climate change, and greater rates and magnitude of climate change increase the likelihood of exceeding adaptation limits (high confidence) (3.3). \{WGI 11.3, 12.4, WGII SPM A-3, SPM B-2, SPM C-2, 1.1.4.4, 2.5, 16.3-16.6, 17.3, 19.2, 20.2.3, 20.3, 20.6\}

Without additional mitigation efforts beyond those in place today, and even with adaptation, warming by the end of the 21 st century will lead to high to very high risk of severe, widespread and irreversible impacts globally (high confidence) (Topic 2 and Figure 3.1a). Estimates of warming in 2100 without additional climate mitigation efforts are from $3.7^{\circ} \mathrm{C}$ to $4.8^{\circ} \mathrm{C}$ compared with pre-industrial levels (median climate response); the range is $2.5^{\circ} \mathrm{C}$ to $7.8^{\circ} \mathrm{C}$ when using the 5 th to 95 th percentile range of the median climate response (Figure 3.1). The risks associated with temperatures at or above $4^{\circ} \mathrm{C}$ include severe and widespread impacts on unique and threatened systems, substantial species extinction, large risks to global and regional food security, consequential constraints on common human activities, increased likelihood of triggering tipping points (critical thresholds) and limited potential for adaptation in some cases (high confidence). Some risks of climate change, such as risks to unique and threatened systems and risks associated with extreme weather events, are moderate to high at temperatures $1^{\circ} \mathrm{C}$ to $2^{\circ} \mathrm{C}$ above pre-industrial levels. \{WGII SPM B-1, SPM C-2, WGIII SPM.3\}

Substantial cuts in GHG emissions over the next few decades can substantially reduce risks of climate change by limiting warming in the second half of the 21 st century and beyond (high confidence). Global mean surface warming is largely determined by cumulative emissions, which are, in turn, linked to emissions over different timescales (Figure 3.1). Limiting risks across Reasons For Concern would imply a limit for cumulative emissions of $\mathrm{CO}_{2}$. 
(a) Risks from climate change...

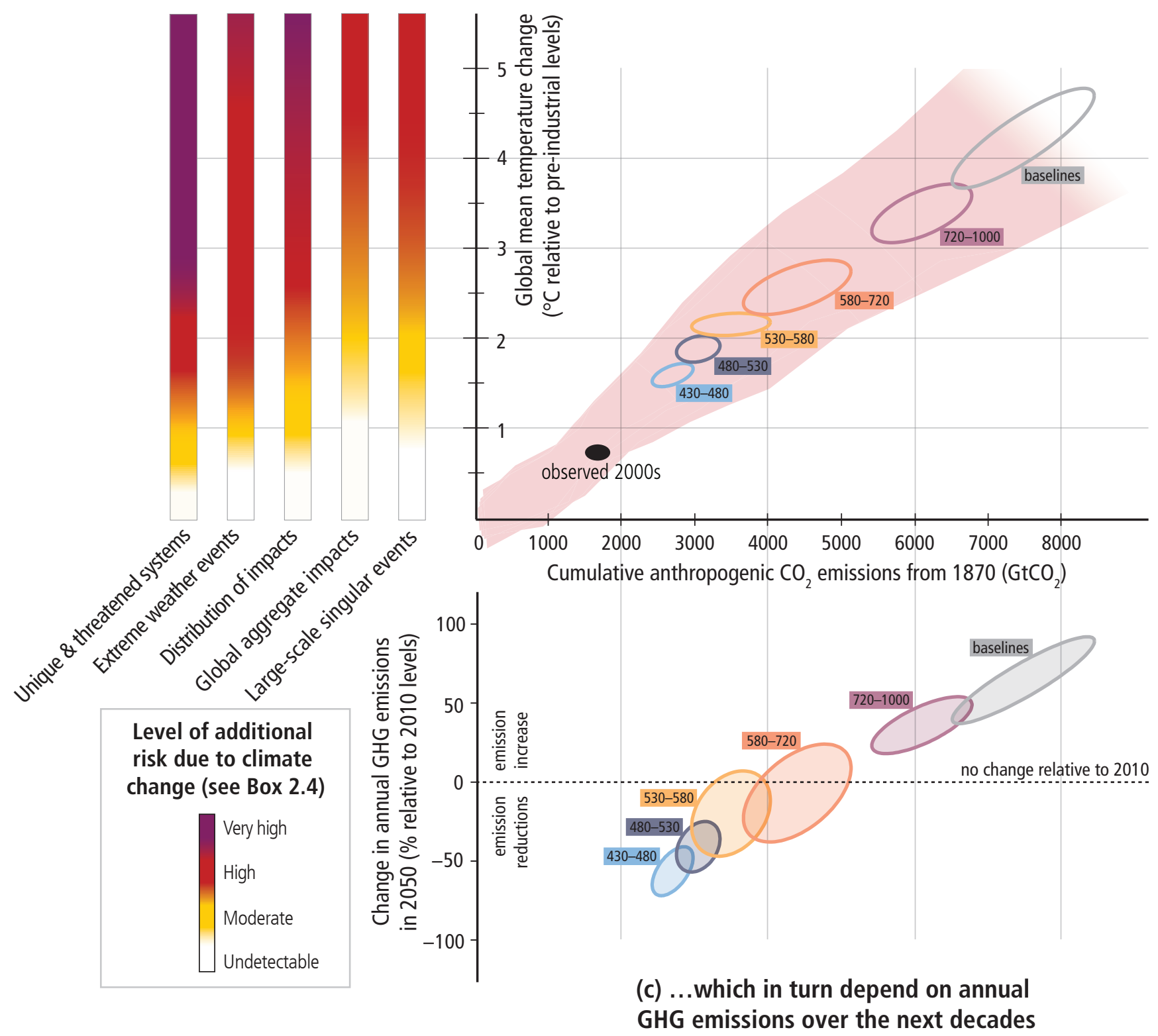

Figure 3.1 | The relationship between risks from climate change, temperature change, cumulative carbon dioxide $\left(\mathrm{CO}_{2}\right)$ emissions and changes in annual greenhouse gas (GHG) emissions by 2050. Limiting risks across Reasons For Concern (a) would imply a limit for cumulative emissions of $\mathrm{CO}_{2}(\mathbf{b})$, which would constrain annual emissions over the next few decades (c). Panel a reproduces the five Reasons For Concern (Box 2.4). Panel b links temperature changes to cumulative $\mathrm{CO}_{2}$ emissions (in $\mathrm{GtCO}_{2}$ ), from 1870 . They are based on Coupled Model Intercomparison Project Phase 5 (CMIP5) simulations (pink plume) and on a simple climate model (median climate response in 2100) for the baselines and five

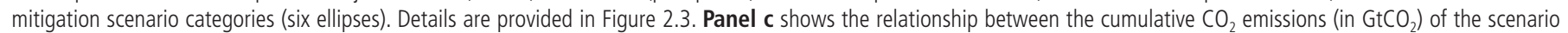
categories and their associated change in annual GHG emissions by 2050, expressed in percentage change (in percent $\mathrm{GtCO}_{2}$-eq per year) relative to 2010. The ellipses correspond to the same scenario categories as in Panel b, and are built with a similar method (see details in Figure 2.3).

Such a limit would require that global net emissions of $\mathrm{CO}_{2}$ eventually decrease to zero (Figure 3.1a,b) (high confidence). Reducing risks of climate change through mitigation would involve substantial cuts in GHG emissions over the next few decades (Figure 3.1c). But some risks from residual damages are unavoidable, even with mitigation and adaptation (very high confidence). A subset of relevant climate change risks has been estimated using aggregate economic indicators. Such economic estimates have important limitations and are therefore a useful but insufficient basis for decision-making on long-term mitigation targets (see Box 3.1). \{WGII 19.7.1, WGIII SPM.3, Figure 3.1$\}$
Mitigation involves some level of co-benefits and risks, but these risks do not involve the same possibility of severe, widespread and irreversible impacts as risks from climate change (high confidence). Scenarios that are likely to limit warming to below $2^{\circ} \mathrm{C}$ or even $3^{\circ} \mathrm{C}$ compared with pre-industrial temperatures involve large-scale changes in energy systems and potentially land use over the coming decades (3.4). Associated risks include those linked to large-scale deployment of technology options for producing low-carbon energy, the potential for high aggregate economic costs of mitigation and impacts on vulnerable countries and industries. Other risks and co-benefits are associated with human health, food security, energy security, poverty 
reduction, biodiversity conservation, water availability, income distribution, efficiency of taxation systems, labour supply and employment, urban sprawl, fossil fuel export revenues and the economic growth of developing countries (Table 4.5). \{WGIII SPM.4.1, SPM.4.2, TS.3.1.4, Table TS.4, Table TS.5, Table TS.6, Table TS.7, Table TS.8, 6.6\}

Inertia in the economic and climate systems and the possibility of irreversible impacts from climate change increase the benefits of near-term mitigation efforts (high confidence). The actions taken today affect the options available in the future to reduce emissions, limit temperature change and adapt to climate change. Near-term choices can create, amplify or limit significant elements of lock-in that are important for decision-making. Lock-ins and irreversibilities occur in the climate system due to large inertia in some of its components such as heat transfer from the ocean surface to depth leading to continued ocean warming for centuries regardless of emission scenario and the irreversibility of a large fraction of anthropogenic climate change resulting from $\mathrm{CO}_{2}$ emissions on a multi-century to millennial timescale unless $\mathrm{CO}_{2}$ were to be removed from the atmosphere through large-scale human interventions over a sustained period (see also Box 3.3). Irreversibilities in socio-economic and biological systems also result from infrastructure development and long-lived products and from climate change impacts, such as species extinction. The larger potential for irreversibility and pervasive impacts from climate change risks than from mitigation risks increases the benefit of shortterm mitigation efforts. Delays in additional mitigation or constraints on technological options limit the mitigation options and increase the long-term mitigation costs as well as other risks that would be incurred in the medium to long term to hold climate change impacts at a given level (Table WGIII SPM.2, blue segment). \{WGI SPM E-8, WGII SPM B-2, 2.1, 19.7, 20.3, Box 20-4, WGIII SPM.4.1, SPM.4.2.1, 3.6, 6.4, 6.6, 6.9\}

\subsection{Characteristics of adaptation pathways}

Adaptation can reduce the risks of climate change impacts, but there are limits to its effectiveness, especially with greater magnitudes and rates of climate change. Taking a longer-term perspective, in the context of sustainable development, increases the likelihood that more immediate adaptation actions will also enhance future options and preparedness.

Adaptation can contribute to the well-being of current and future populations, the security of assets and the maintenance of ecosystem goods, functions and services now and in the future. Adaptation is place- and context-specific, with no single approach for reducing risks appropriate across all settings (high confidence). Effective risk reduction and adaptation strategies consider vulnerability and exposure and their linkages with socio-economic processes, sustainable development, and climate change. Adaptation research since the IPCC Fourth Assessment Report (AR4) has evolved from a dominant consideration of engineering and technological adaptation pathways to include more ecosystem-based, institutional and social measures. A previous focus on cost-benefit analysis, optimization and efficiency approaches has broadened with the development of multi-metric evaluations that include risk and uncertainty dimensions integrated within wider policy and ethical frameworks to assess tradeoffs and constraints. The range of specific adaptation measures has also expanded $(4.2,4.4 .2 .1)$, as have the links to sustainable development (3.5). There are many studies on local and sectoral adaptation costs and benefits, but few global analyses and very low confidence

\section{Box 3.1 | The Limits of the Economic Assessment of Climate Change Risks}

A subset of climate change risks and impacts are often measured using aggregate economic indicators, such as gross domestic product (GDP) or aggregate income. Estimates, however, are partial and affected by important conceptual and empirical limitations. These incomplete estimates of global annual economic losses for temperature increases of $\sim 2.5^{\circ} \mathrm{C}$ above pre-industrial levels are between 0.2 and $2.0 \%$ of income (medium evidence, medium agreement). Losses are more likely than not to be greater, rather than smaller, than this range (limited evidence, high agreement). Estimates of the incremental aggregate economic impact of emitting one more tonne of carbon dioxide (the social cost of carbon) are derived from these studies and lie between a few dollars and several hundreds of dollars per tonne of carbon in 2000 to 2015 (robust evidence, medium agreement). These impact estimates are incomplete and depend on a large number of assumptions, many of which are disputable. Many estimates do not account for the possibility of large-scale singular events and irreversibility, tipping points and other important factors, especially those that are difficult to monetize, such as loss of biodiversity. Estimates of aggregate costs mask significant differences in impacts across sectors, regions, countries and communities, and they therefore depend on ethical considerations, especially on the aggregation of losses across and within countries (high confidence). Estimates of global aggregate economic losses exist only for limited warming levels. These levels are exceeded in scenarios for the 21 st century unless additional mitigation action is implemented, leading to additional economic costs. The total economic effects at different temperature levels would include mitigation costs, co-benefits of mitigation, adverse side effects of mitigation, adaptation costs and climate damages. As a result, mitigation cost and climate damage estimates at any given temperature level cannot be compared to evaluate the costs and benefits of mitigation. Very little is known about the economic cost of warming above $3^{\circ} \mathrm{C}$ relative to the current temperature level. Accurately estimating climate change risks (and thus the benefits of mitigation) takes into account the full range of possible impacts of climate change, including those with high consequences but a low probability of occurrence. The benefits of mitigation may otherwise be underestimated (high confidence). Some limitations of current estimates may be unavoidable, even with more knowledge, such as issues with aggregating impacts over time and across individuals when values are heterogeneous. In view of these limitations, it is outside the scope of science to identify a single best climate change target and climate policy (3.1, 3.4). \{WGII SPM B-2, 10.9.2, 10.9.4, 13.2, 17.2-17.3, 18.4, 19.6, WGIII 3.6\} 
in their results. \{WGII SPM C-1, Table SPM.1, 14.1, 14.ES, 15.2, 15.5, 17.2, 17.ES\}

Adaptation planning and implementation at all levels of governance are contingent on societal values, objectives and risk perceptions (high confidence). Recognition of diverse interests, circumstances, social-cultural contexts and expectations can benefit decision-making processes. Indigenous, local and traditional knowledge systems and practices, including indigenous peoples' holistic view of community and environment, are a major resource for adapting to climate change, but these have not been used consistently in existing adaptation efforts. Integrating such forms of knowledge into practices increases the effectiveness of adaptation as do effective decision support, engagement and policy processes (4.4.2). \{WGII SPM C-1\}

Adaptation planning and implementation can be enhanced through complementary actions across levels, from individuals to governments (high confidence). National governments can coordinate adaptation efforts of local and sub-national governments, for example by protecting vulnerable groups, by supporting economic diversification and by providing information, policy and legal frameworks and financial support (robust evidence, high agreement). Local government and the private sector are increasingly recognized as critical to progress in adaptation, given their roles in scaling up adaptation of communities, households and civil society and in managing risk information and financing (medium evidence, high agreement). \{WGII SPM C-1\}

A first step towards adaptation to future climate change is reducing vulnerability and exposure to present climate variability (high confidence), but some near-term responses to climate change may also limit future choices. Integration of adaptation into planning, including policy design, and decision-making can promote synergies with development and disaster risk reduction. However, poor planning or implementation, overemphasizing short-term outcomes or failing to sufficiently anticipate consequences can result in maladaptation, increasing the vulnerability or exposure of the target group in the future or the vulnerability of other people, places or sectors (medium evidence, high agreement). For example, enhanced protection of exposed assets can lock in dependence on further protection measures. Appropriate adaptation options can be better assessed by including co-benefits and mitigation implications (3.5 and 4.2). \{WGIl SPM C-1\}

Numerous interacting constraints can impede adaptation planning and implementation (high confidence). Common constraints on implementation arise from the following: limited financial and human resources; limited integration or coordination of governance; uncertainties about projected impacts; different perceptions of risks; competing values; absence of key adaptation leaders and advocates; and limited tools to monitor adaptation effectiveness. Other constraints include insufficient research, monitoring and observation and the financial and other resources to maintain them. Underestimating the complexity of adaptation as a social process can create unrealistic expectations about achieving intended adaptation outcomes (see Sections 4.1 and 4.2 for details in relation to implementation). \{WGII SPM C-1\}
Greater rates and magnitude of climate change increase the likelihood of exceeding adaptation limits (high confidence). Limits to adaptation occur when adaptive actions to avoid intolerable risks for an actor's objectives or for the needs of a system are not possible or are not currently available. Value-based judgments of what constitutes an intolerable risk may differ. Limits to adaptation emerge from the interaction among climate change and biophysical and/or socio-economic constraints. Opportunities to take advantage of positive synergies between adaptation and mitigation may decrease with time, particularly if limits to adaptation are exceeded. In some parts of the world, insufficient responses to emerging impacts are already eroding the basis for sustainable development. For most regions and sectors, empirical evidence is not sufficient to quantify magnitudes of climate change that would constitute a future adaptation limit. Furthermore, economic development, technology and cultural norms and values can change over time to enhance or reduce the capacity of systems to avoid limits. As a consequence, some limits are 'soft' in that they may be alleviated over time. Other limits are 'hard' in that there are no reasonable prospects for avoiding intolerable risks. \{WGII SPM C-2, TS\}

Transformations in economic, social, technological and political decisions and actions can enhance adaptation and promote sustainable development (high confidence). Restricting adaptation responses to incremental changes to existing systems and structures without considering transformational change may increase costs and losses and miss opportunities. For example, enhancing infrastructure to protect other built assets can be expensive and ultimately not defray increasing costs and risks, whereas options such as relocation or using ecosystem services to adapt may provide a range of benefits now and in the future. Transformational adaptation can include introduction of new technologies or practices, formation of new financial structures or systems of governance, adaptation at greater scales or magnitudes and shifts in the location of activities. Planning and implementation of transformational adaptation could reflect strengthened, altered or aligned paradigms and consequently may place new and increased demands on governance structures to reconcile different goals and visions for the future and to address possible equity and ethical implications: transformational adaptation pathways are enhanced by iterative learning, deliberative processes, and innovation. At the national level, transformation is considered most effective when it reflects a country's own visions and approaches to achieving sustainable development in accordance with its national circumstances and priorities. \{WGII SPM C-2, 1.1, 2.5, 5.5, 8.4, 14.1, 14.3, 16.2-7, 20.3.3, 20.5, 25.10, Table 14-4, Table 16-3, Box 16.1, Box 16.4, Box 25.1\}

Building adaptive capacity is crucial for effective selection and implementation of adaptation options (robust evidence, high agreement). Successful adaptation requires not only identifying adaptation options and assessing their costs and benefits, but also increasing the adaptive capacity of human and natural systems (medium evidence, high agreement). This can involve complex governance challenges and new institutions and institutional arrangements. (4.2) $\{W G / l$ 8.1, 12.3, 14.1-3, 16.2, 16.3, 16.5, 16.8\}

Significant co-benefits, synergies and trade-offs exist between mitigation and adaptation and among different adaptation responses; interactions occur both within and across regions (very high confidence). Increasing efforts to mitigate and adapt to climate 
change imply an increasing complexity of interactions, particularly at the intersections among water, energy, land use and biodiversity, but tools to understand and manage these interactions remain limited. Examples of actions with co-benefits include (i) improved energy efficiency and cleaner energy sources, leading to reduced emissions of health-damaging, climate-altering air pollutants; (ii) reduced energy and water consumption in urban areas through greening cities and recycling water; (iii) sustainable agriculture and forestry; and (iv) protection of ecosystems for carbon storage and other ecosystem services. \{WGII SPM C-1\}

\subsection{Characteristics of mitigation pathways}

There are multiple mitigation pathways that are likely to limit warming to below $2^{\circ} \mathrm{C}$ relative to pre-industrial levels. These pathways would require substantial emissions reductions over the next few decades and near zero emissions of $\mathrm{CO}_{2}$ and other long-lived greenhouse gases by the end of the century. Implementing such reductions poses substantial technological, economic, social and institutional challenges, which increase with delays in additional mitigation and if key technologies are not available. Limiting warming to lower or higher levels involves similar challenges but on different timescales.

Without additional efforts to reduce GHG emissions beyond those in place today, global emission growth is expected to persist driven by growth in global population and economic activities (high confidence) (Figure 3.2). Global GHG emissions under most scenarios without additional mitigation (baseline scenarios) are between about $75 \mathrm{GtCO}_{2}$-eq/yr and almost $140 \mathrm{GtCO}_{2}$-eq/yr in $2100^{35}$ which is approximately between the 2100 emission levels in the RCP6.0 and RCP8.5 pathways (Figure 3.2) ${ }^{36}$. Baseline scenarios exceed 450 ppm $\mathrm{CO}_{2}$-eq by 2030 and reach $\mathrm{CO}_{2}$-eq concentration levels between about 750 ppm $\mathrm{CO}_{2}$-eq and more than 1300 ppm $\mathrm{CO}_{2}$-eq by 2100. Global mean surface temperature increases in 2100 range from about $3.7^{\circ} \mathrm{C}$ to $4.8^{\circ} \mathrm{C}$ above the average for $1850-1900$ for a median climate response. They range from $2.5^{\circ} \mathrm{C}$ to $7.8^{\circ} \mathrm{C}$ when including climate uncertainty ( 5 th to 95 th percentile range) ${ }^{37}$. The future scenarios do not account for possible changes in natural forcings in the climate system (see Box 1.1). \{WGIII SPM.3, SPM.4.1, TS.2.2, TS.3.1, 6.3, Box TS.6\}
Many different combinations of technological, behavioural and policy options can be used to reduce emissions and limit temperature change (high confidence). To evaluate possible pathways to long-term climate goals, about 900 mitigation scenarios were collected for this assessment, each of which describes different technological, socio-economic and institutional changes. Emission reductions under these scenarios lead to concentrations in 2100 from $430 \mathrm{ppm}$ $\mathrm{CO}_{2}$-eq to above $720 \mathrm{ppm} \mathrm{CO}$-eq which is comparable to the 2100 forcing levels between RCP2.6 and RCP6.0. Scenarios with concentration levels of below 430 ppm $\mathrm{CO}_{2}$-eq by 2100 were also assessed. \{WGIII SPM.4.1, TS3.1, 6.1, 6.2, 6.3, Annex II\}

Scenarios leading to $\mathrm{CO}_{2}$-eq concentrations in 2100 of about $450 \mathrm{ppm}$ or lower are likely to maintain warming below $2^{\circ} \mathrm{C}$ over the 21st century relative to pre-industrial levels (high confidence). Mitigation scenarios reaching concentration levels of about 500 ppm $\mathrm{CO}_{2}$-eq by 2100 are more likely than not to limit warming to less than $2^{\circ} \mathrm{C}$ relative to pre-industrial levels, unless concentration levels temporarily exceed roughly $530 \mathrm{ppm} \mathrm{CO}_{2}$-eq before 2100 . In this case, warming is about as likely as not to remain below $2{ }^{\circ} \mathrm{C}$ relative to pre-industrial levels. Scenarios that exceed about 650 ppm $\mathrm{CO}_{2}$-eq by 2100 are unlikely to limit warming to below $2^{\circ} \mathrm{C}$ relative to pre-industrial levels. Mitigation scenarios in which warming is more likely than not to be less than $1.5^{\circ} \mathrm{C}$ relative to pre-industrial levels by 2100 are characterized by concentration levels by 2100 of below 430 ppm $\mathrm{CO}_{2}$-eq. In these scenarios, temperature peaks during the century and subsequently declines (Table 3.1). \{WGIII SPM.4.1, Table SPM.1, TS.3.1, Box TS.6, 6.3\}

Mitigation scenarios reaching about 450 ppm CO $\mathrm{CO}_{2}$-eq in 2100 (consistent with a likely chance to keep warming below $2^{\circ} \mathrm{C}$ relative to pre-industrial level) typically involve temporary overshoot $^{38}$ of atmospheric concentrations, as do many scenarios reaching about 500 ppm $\mathrm{CO}_{2}$-eq to about 550 ppm $\mathrm{CO}_{2}$-eq by 2100 (Table 3.1). Depending on the level of overshoot, overshoot scenarios typically rely on the availability and widespread deployment of bioenergy with carbon dioxide capture and storage (BECCS) and afforestation in the second half of the century (high confidence). The availability and scale of these and other Carbon Dioxide Removal (CDR) technologies and methods are uncertain, and CDR technologies and methods are, to varying degrees, associated with challenges and risks (see Box 3.3) ${ }^{39}$. CDR is also prevalent in many scenarios without overshoot to compensate for residual emissions from sectors where mitigation is more expensive. \{WGIII SPM.4.1, Table SPM.1, TS.3.1, 6.3, 6.9.1, Figure 6.7, 7.11, 11.13\}

35 Unless otherwise noted, scenario ranges cited in Topic 3 and Topic 4 refer to the 10th to 90th percentile ranges (see Table 3.1).

36 For a discussion on CO2-equivalent (CO2-eq) emissions and concentrations, see Box 3.2 on GHG metrics and mitigation pathways and the Glossary.

37 The range quoted here is based on the warming results of a simple climate model for the emissions of around 300 baseline scenarios, expressed compared to the $1850-1900$ period. The warming results quoted in Section 2.2 are obtained by prescribing future concentrations of GHG in CMIP5 Earth System Models. This results in a mean warming of $1.0^{\circ} \mathrm{C}$ (5th to 95 th percentile range: $0.3^{\circ} \mathrm{C}$ to $1.7^{\circ} \mathrm{C}$ ) for $\mathrm{RCP} 2.6$, and a mean warming of $3.7^{\circ} \mathrm{C}\left(2.6^{\circ} \mathrm{C}\right.$ to $\left.4.8^{\circ} \mathrm{C}\right)$ for $\mathrm{RCP} 8.5^{2}$ relative to the period $1986-2005$. For the same concentration-driven experiments, the simple climate model approach gives consistent results. The median warming is $0.9^{\circ} \mathrm{C}\left(0.5^{\circ} \mathrm{C}\right.$ to $\left.1.6^{\circ} \mathrm{C}\right)$ for $\mathrm{RCP} 2.6$ and $3.7^{\circ} \mathrm{C}\left(2.5^{\circ} \mathrm{C}\right.$ to $5.9^{\circ} \mathrm{C}$ ) for RCP8.5 relative to the period 1986-2005. However, the high-end of the CMIP5 ESMs range is more constrained. In addition, the baseline temperature increase quoted here is wider than that of the concentration-driven RCP8.5 experiments mentioned above as it is based on a wider set of scenarios, includes carbon cycle response uncertainty, and uses a different base year $(2.2,3.4)$

38 In concentration 'overshoot' scenarios, concentrations peak during the century and then decline.

39 CDR methods have biogeochemical and technological limitations to their potential on the global scale. There is insufficient knowledge to quantify how much CO2 emissions could be partially offset by CDR on a century timescale. CDR methods may carry side effects and long-term consequences on a global scale. 
(a) GHG emission pathways 2000-2100: All AR5 scenarios

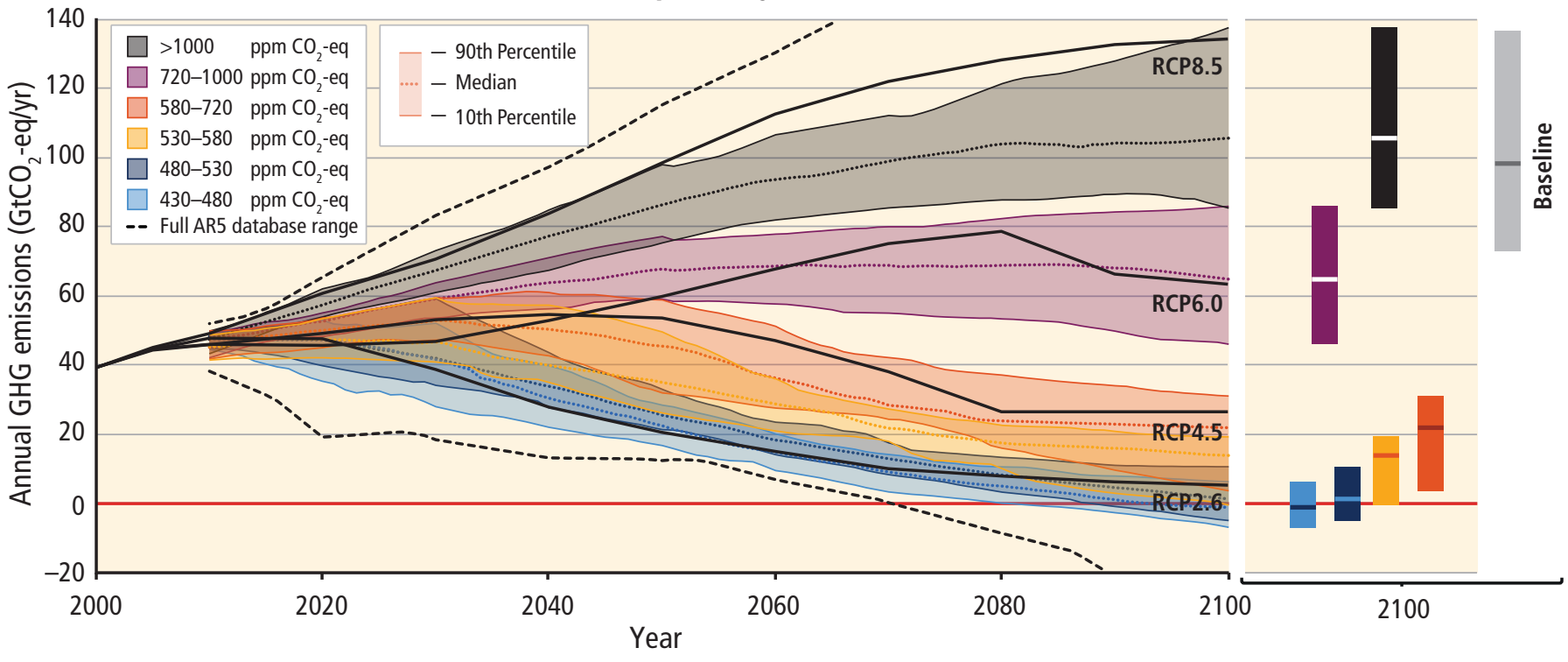

(b)

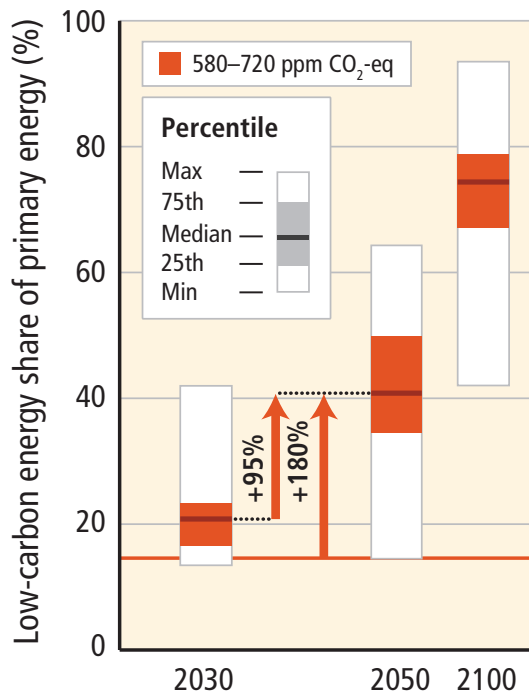

Associated upscaling of low-carbon energy supply
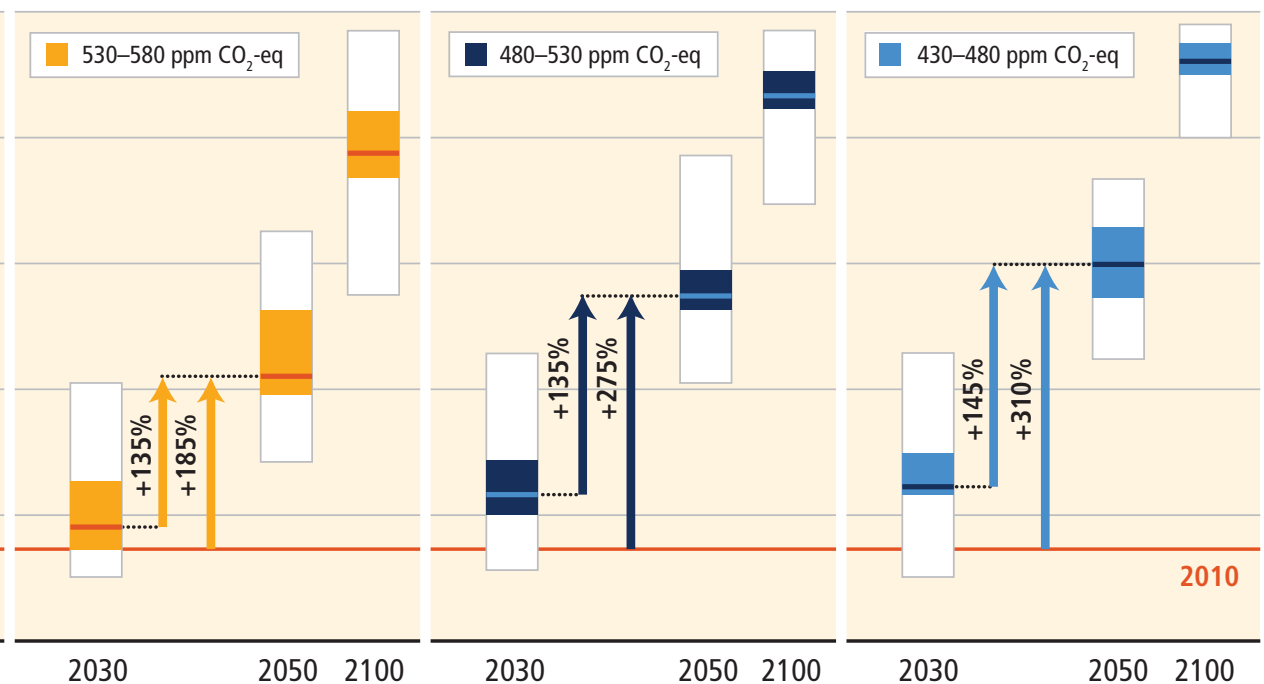

Figure 3.2 2 Global greenhouse gas (GHG) emissions (gigatonne of $\mathrm{CO}_{2}$-equivalent per year, $\mathrm{GtCO}_{2}$-eq/yr) in baseline and mitigation scenarios for different long-term concentration levels (a) and associated scale-up requirements of low-carbon energy (\% of primary energy) for 2030, 2050 and 2100, compared to 2010 levels, in mitigation scenarios (b). \{WGIII SPM.4, Figure 6.7, Figure 7.16] [Note: $\mathrm{CO}_{2}$-eq emissions include the basket of Kyoto gases (carbon dioxide $\left(\mathrm{CO}_{2}\right)$, methane $\left(\mathrm{CH}_{4}\right)$, nitrous oxide $\left(\mathrm{N}_{2} \mathrm{O}\right)$ as well as fluorinated gases) calculated based on 100-year Global Warming Potential (GWP 100$)$ values from the IPCC Second Assessment Report.]

Limiting warming with a likely chance to less than $2^{\circ} \mathrm{C}$ relative to pre-industrial levels would require substantial cuts in anthropogenic GHG emissions ${ }^{40}$ by mid-century through largescale changes in energy systems and possibly land use. Limiting warming to higher levels would require similar changes but less quickly. Limiting warming to lower levels would require these changes more quickly (high confidence). Scenarios that are likely to maintain warming at below $2{ }^{\circ} \mathrm{C}$ are characterized by a 40 to $70 \%$ reduction in GHG emissions by 2050, relative to 2010 levels, and emissions levels near zero or below in 2100 (Figure 3.2, Table 3.1). Scenarios with higher emissions in 2050 are characterized by a greater reliance on CDR technologies beyond mid-century, and vice versa. Scenarios that are likely to maintain warming at below $2^{\circ} \mathrm{C}$ include more rapid improvements in energy efficiency and a tripling to nearly a quadrupling of the share of zero- and low-carbon energy supply from renewable energy, nuclear energy and fossil energy with carbon dioxide capture and storage (CCS) or BECCS by the year 2050 (Figure 3.2b). The scenarios describe a wide range of changes in land use, reflecting

40 This range differs from the range provided for a similar concentration category in AR4 (50 to 85\% lower than in 2000 for $\mathrm{CO}_{2}$ only). Reasons for this difference include that this report has assessed a substantially larger number of scenarios than in AR4 and looks at all GHGs. In addition, a large proportion of the new scenarios include CDR technologies. Other factors include the use of 2100 concentration levels instead of stabilization levels and the shift in reference year from 2000 to 2010 . Scenarios with higher emission levels by 2050 are characterized by a greater reliance on CDR technologies beyond mid-century. 
Table 3.1 | Key characteristics of the scenarios collected and assessed for WGIII AR5. For all parameters the 10th to 90th percentile of the scenarios is shown ${ }^{\text {a }}$

\begin{tabular}{|c|c|c|c|c|c|c|c|c|}
\hline \multirow{2}{*}{$\begin{array}{l}\mathrm{CO}_{2} \text {-eq Con- } \\
\text { centrations in } \\
2100 \\
\left(\mathrm{ppm} \mathrm{CO} \mathrm{CO}_{2}-\mathrm{eq}\right)^{\mathrm{f}} \\
\text { Category label } \\
\text { (conc. range) }\end{array}$} & \multirow[b]{2}{*}{ Subcategories } & \multirow{2}{*}{$\begin{array}{l}\text { Relative } \\
\text { position of } \\
\text { the RCPs d }\end{array}$} & \multicolumn{2}{|c|}{$\begin{array}{l}\text { Change in } \mathrm{CO}_{2} \text {-eq emissions } \\
\text { compared to } 2010 \text { (in \%) }\end{array}$} & \multicolumn{4}{|c|}{$\begin{array}{l}\text { Likelihood of staying below a specific temperature level } \\
\text { over the } 21 \text { st century (relative to } 1850-1900 \text { ) d, e }\end{array}$} \\
\hline & & & 2050 & 2100 & $1.5^{\circ} \mathrm{C}$ & $2^{\circ} \mathrm{C}$ & $3^{\circ} \mathrm{C}$ & $4^{\circ} \mathrm{C}$ \\
\hline$<430$ & \multicolumn{8}{|c|}{ Only a limited number of individual model studies have explored levels below $430 \mathrm{ppm} \mathrm{CO}_{2}$-eq ${ }^{\mathrm{j}}$} \\
\hline \multirow{2}{*}{$\begin{array}{c}500 \\
(480 \text { to } 530)\end{array}$} & $\begin{array}{l}\text { No overshoot of } \\
530 \mathrm{ppm} \mathrm{CO}_{2} \text {-eq }\end{array}$ & & -57 to -42 & -107 to -73 & \multirow{4}{*}{ Unlikely } & $\begin{array}{c}\text { More likely } \\
\text { than not }\end{array}$ & \multirow{3}{*}{ Likely } & \multirow{5}{*}{ Likely } \\
\hline & $\begin{array}{c}\text { Overshoot of } 530 \\
\text { ppm } \mathrm{CO}_{2} \text {-eq }\end{array}$ & & -55 to -25 & -114 to -90 & & $\begin{array}{c}\text { About as } \\
\text { likely as not }\end{array}$ & & \\
\hline $\begin{array}{c}550 \\
(530 \text { to } 580)\end{array}$ & $\begin{array}{l}\text { No overshoot of } \\
580 \mathrm{ppm} \mathrm{CO}_{2} \text {-eq }\end{array}$ & & -47 to -19 & -81 to -59 & & $\begin{array}{c}\text { More unlikely } \\
\text { than likely }{ }^{i}\end{array}$ & & \\
\hline (650 to 720 ) & Total range & $\mathrm{RCP} 4.5$ & -11 to 17 & -54 to -21 & & \multirow{2}{*}{ Unlikely } & $\begin{array}{c}\text { More likely } \\
\text { than not }\end{array}$ & \\
\hline$(720 \text { to } 1000)^{b}$ & Total range & RCP6.0 & 18 to 54 & -7 to 72 & \multirow{2}{*}{ Unlikely ${ }^{h}$} & & $\begin{array}{l}\text { More unlikely } \\
\text { than likely }\end{array}$ & \\
\hline$>1000^{b}$ & Total range & RCP8.5 & 52 to 95 & 74 to 178 & & Unlikely ${ }^{h}$ & Unlikely & $\begin{array}{c}\text { More unlikely } \\
\text { than likely }\end{array}$ \\
\hline
\end{tabular}

Notes:

a The 'total range' for the 430 to 480 ppm $\mathrm{CO}_{2}$-eq concentrations scenarios corresponds to the range of the 10th to 90 th percentile of the subcategory of these scenarios shown in Table 6.3 of the Working Group III report.

${ }^{b}$ Baseline scenarios fall into the $>1000$ and 720 to 1000 ppm CO $\mathrm{CO}_{2}$-eq categories. The latter category also includes mitigation scenarios. The baseline scenarios in the latter category reach a temperature change of $2.5^{\circ} \mathrm{C}$ to $5.8^{\circ} \mathrm{C}$ above the average for $1850-1900$ in 2100 . Together with the baseline scenarios in the $>1000$ ppm $\mathrm{CO}_{2}$-eq category, this leads to an overall 2100 temperature range of $2.5^{\circ} \mathrm{C}$ to $7.8^{\circ} \mathrm{C}$ (range based on median climate response: $3.7^{\circ} \mathrm{C}$ to $4.8^{\circ} \mathrm{C}$ ) for baseline scenarios across both concentration categories.

' The global 2010 emissions are $31 \%$ above the 1990 emissions (consistent with the historic greenhouse gas emission estimates presented in this report). CO ${ }_{2}$-eq emissions include the basket of Kyoto gases (carbon dioxide $\left(\mathrm{CO}_{2}\right)$, methane $\left(\mathrm{CH}_{4}\right)$, nitrous oxide $\left(\mathrm{N}_{2} \mathrm{O}\right)$ as well as fluorinated gases).

${ }^{d}$ The assessment here involves a large number of scenarios published in the scientific literature and is thus not limited to the Representative Concentration Pathways (RCPs). To evaluate the $\mathrm{CO}_{2}$-eq concentration and climate implications of these scenarios, the Model for the Assessment of Greenhouse Gas Induced Climate Change (MAGICC) was used in a probabilistic mode. For a comparison between MAGICC model results and the outcomes of the models used in WGI, see WGI 12.4.1.2, 12.4.8 and WGIII 6.3.2.6.

e The assessment in this table is based on the probabilities calculated for the full ensemble of scenarios in WGIII using MAGICC and the assessment in WGI of the uncertainty of the temperature projections not covered by climate models. The statements are therefore consistent with the statements in WGI, which are based on the Coupled Model Intercomparison Project Phase 5 (CMIP5) runs of the RCPs and the assessed uncertainties. Hence, the likelihood statements reflect different lines of evidence from both WGs. This WGI method was also applied for scenarios with intermediate concentration levels where no CMIP5 runs are available. The likelihood statements are indicative only \{WGIII 6.3\} and follow broadly the terms used by the WGI SPM for temperature projections: likely 66-100\%, more likely than not $>50-100 \%$, about as likely as not 33-66\%, and unlikely 0-33\%. In addition the term more unlikely than likely $0-<50 \%$ is used.

${ }^{\mathrm{f}}$ The $\mathrm{CO}_{2}$-equivalent concentration (see Glossary) is calculated on the basis of the total forcing from a simple carbon cycle/climate model, $\mathrm{MAGICC}$. The $\mathrm{CO}_{2}$-equivalent concentration in 2011 is estimated to be 430 ppm (uncertainty range 340 to 520 ppm). This is based on the assessment of total anthropogenic radiative forcing for 2011 relative to 1750 in WGI, i.e., $2.3 \mathrm{~W} / \mathrm{m}^{2}$, uncertainty range 1.1 to $3.3 \mathrm{~W} / \mathrm{m}^{2}$.

$g$ The vast majority of scenarios in this category overshoot the category boundary of 480 ppm $\mathrm{CO}_{2}$-eq concentration.

h For scenarios in this category, no CMIP5 run or MAGICC realization stays below the respective temperature level. Still, an unlikely assignment is given to reflect uncertainties that may not be reflected by the current climate models.

i Scenarios in the 580 to 650 ppm $\mathrm{CO}_{2}$-eq category include both overshoot scenarios and scenarios that do not exceed the concentration level at the high end of the category (e.g., RCP4.5). The latter type of scenarios, in general, have an assessed probability of more unlikely than likely to stay below the $2^{\circ} \mathrm{C}$ temperature level, while the former are mostly assessed to have an unlikely probability of staying below this level.

$\mathrm{j}$ In these scenarios, global $\mathrm{CO}_{2}$-eq emissions in 2050 are between 70 to $95 \%$ below 2010 emissions, and they are between 110 to $120 \%$ below 2010 emissions in 2100 .

different assumptions about the scale of bioenergy production, afforestation and reduced deforestation. Scenarios leading to concentrations of $500 \mathrm{ppm} \mathrm{CO} \mathrm{CO}_{2}$-eq by 2100 are characterized by a 25 to $55 \%$ reduction in GHG emissions by 2050, relative to 2010 levels. Scenarios that are likely to limit warming to $3^{\circ} \mathrm{C}$ relative to pre-industrial levels reduce emissions less rapidly than those limiting warming to $2^{\circ} \mathrm{C}$. Only a limited number of studies provide scenarios that are more likely than not to limit warming to $1.5^{\circ} \mathrm{C}$ by 2100 ; these scenarios are characterized by concentrations below $430 \mathrm{ppm} \mathrm{CO}$-eq by 2100 and 2050 emission reduction between 70 and $95 \%$ below 2010 . For a comprehensive overview of the characteristics of emissions scenarios, their $\mathrm{CO}_{2}$-equivalent concentrations and their likelihood to keep warming to below a range of temperature levels, see Table 3.1. \{WGIII SPM.4.1, $T S .3 .1,6.3,7.11\}$ 

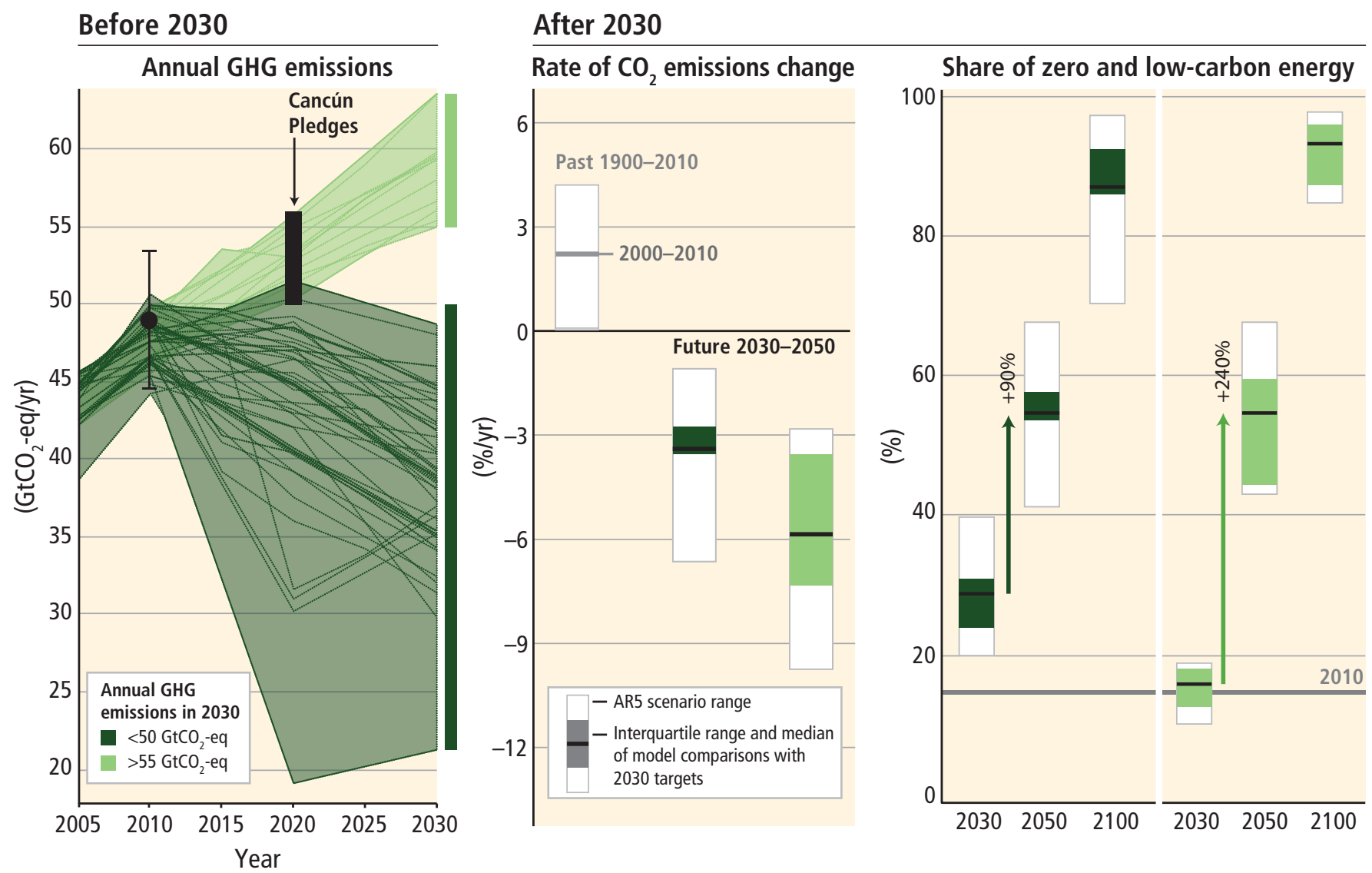

Figure 3.3 The implications of different 2030 greenhouse gas (GHG) emissions levels for the rate of carbon dioxide $\left(\mathrm{CO}_{2}\right)$ emission reductions and low-carbon energy upscaling in mitigation scenarios that are at least about as likely as not to keep warming throughout the 21 st century below $2^{\circ} \mathrm{C}$ relative to pre-industrial levels $\left(2100 \mathrm{CO}_{2}\right.$-eq concentrations 430 to 530 ppm). The scenarios are grouped according to different emissions levels by 2030 (coloured in different shades of green). The left panel shows the pathways of GHG emissions $\left(\mathrm{GtCO}_{2}\right.$-eq/yr) leading to these 2030 levels. Black dot with whiskers gives historic $\mathrm{GHG}$ emission levels and associated uncertainties in 2010 as reported in Figure 1.6. The black bar shows the estimated uncertainty range of $\mathrm{GHG}$ emissions implied by the Cancún Pledges. The middle panel denotes the average annual $\mathrm{CO}_{2}$ emission reduction rates for the 2030-2050 period. It compares the median and interquartile range across scenarios from recent intermodel comparisons with explicit 2030 interim goals to the range of scenarios in the Scenario Database for WGIII AR5. Annual rates of historical emission changes (sustained over a period of 20 years) are shown as well. The arrows in the right panel show the magnitude of zero and low-carbon energy supply upscaling from between 2030 and 2050, subject to different 2030 GHG emission levels. Zero- and low-carbon energy supply includes renewable energy, nuclear energy and fossil energy with carbon dioxide capture and storage (CCS) or bioenergy with CCS (BECCS). Only scenarios that apply the full, unconstrained mitigation technology portfolio of the underlying models (default technology assumption) are shown. Scenarios with large net negative global emissions (>20 $\mathrm{GtCO}_{2}$-eq/yr), scenarios with exogenous carbon price assumptions, and scenarios with 2010 emission levels that are significantly outside the historical range are excluded. \{WGIII Figure SPM.5, Figure 6.32, Figure 7.16, 13.13.1.3\}

Reducing emissions of non- $\mathrm{CO}_{2}$ climate forcing agents can be an important element of mitigation strategies. Emissions of non$\mathrm{CO}_{2}$ gases (methane $\left(\mathrm{CH}_{4}\right)$, nitrous oxide $\left(\mathrm{N}_{2} \mathrm{O}\right)$, and fluorinated gases) contributed about $27 \%$ to the total emissions of Kyoto gases in 2010. For most non- $\mathrm{CO}_{2}$ gases, near-term, low-cost options are available to reduce their emissions. However, some sources of these non- $\mathrm{CO}_{2}$ gases are difficult to mitigate, such as $\mathrm{N}_{2} \mathrm{O}$ emissions from fertilizer use and $\mathrm{CH}_{4}$ emissions from livestock. As a result, emissions of most non- $\mathrm{CO}_{2}$ gases will not be reduced to zero, even under stringent mitigation scenarios (see Figure 4.1). The differences in radiative properties and lifetimes of $\mathrm{CO}_{2}$ and non- $\mathrm{CO}_{2}$ climate forcing agents have important implications for mitigation strategies (see also Box 3.2). \{WGIII 6.3.2\}

All current GHG emissions and other climate forcing agents affect the rate and magnitude of climate change over the next few decades. Reducing the emissions of certain short-lived climate forcing agents can reduce the rate of warming in the short term but will have only a limited effect on long-term warming, which is driven mainly by $\mathrm{CO}_{2}$ emissions. There are large uncertainties related to the climate impacts of some of the short-lived climate forcing agents. Although the effects of $\mathrm{CH}_{4}$ emissions are well understood, there are large uncertainties related to the effects of black carbon. Co-emitted components with cooling effects may further complicate and reduce the climate impacts of emission reductions. Reducing emissions of sulfur dioxide $\left(\mathrm{SO}_{2}\right)$ would cause warming. Near-term reductions in short-lived climate forcing agents can have a relatively fast impact on climate change and possible co-benefits for air pollution. $\{W G I$ 8.2.3, 8.3.2, 8.3.4, 8.5.1, 8.7.2, FAQ 8.2, 12.5, WGIII 6.6.2.1\}

Delaying additional mitigation to 2030 will substantially increase the challenges associated with limiting warming over the 21st century to below $2^{\circ} \mathrm{C}$ relative to pre-industrial levels (high confidence). GHG emissions in 2030 lie between about $30 \mathrm{GtCO}_{2}$-eq/yr and $50 \mathrm{GtCO}_{2}$-eq/yr in cost-effective scenarios that are likely to about as likely as not to limit warming to less than $2^{\circ} \mathrm{C}$ this century relative to pre-industrial levels (2100 atmospheric concentration 


\section{Global mitigation costs and consumption growth in baseline scenarios}
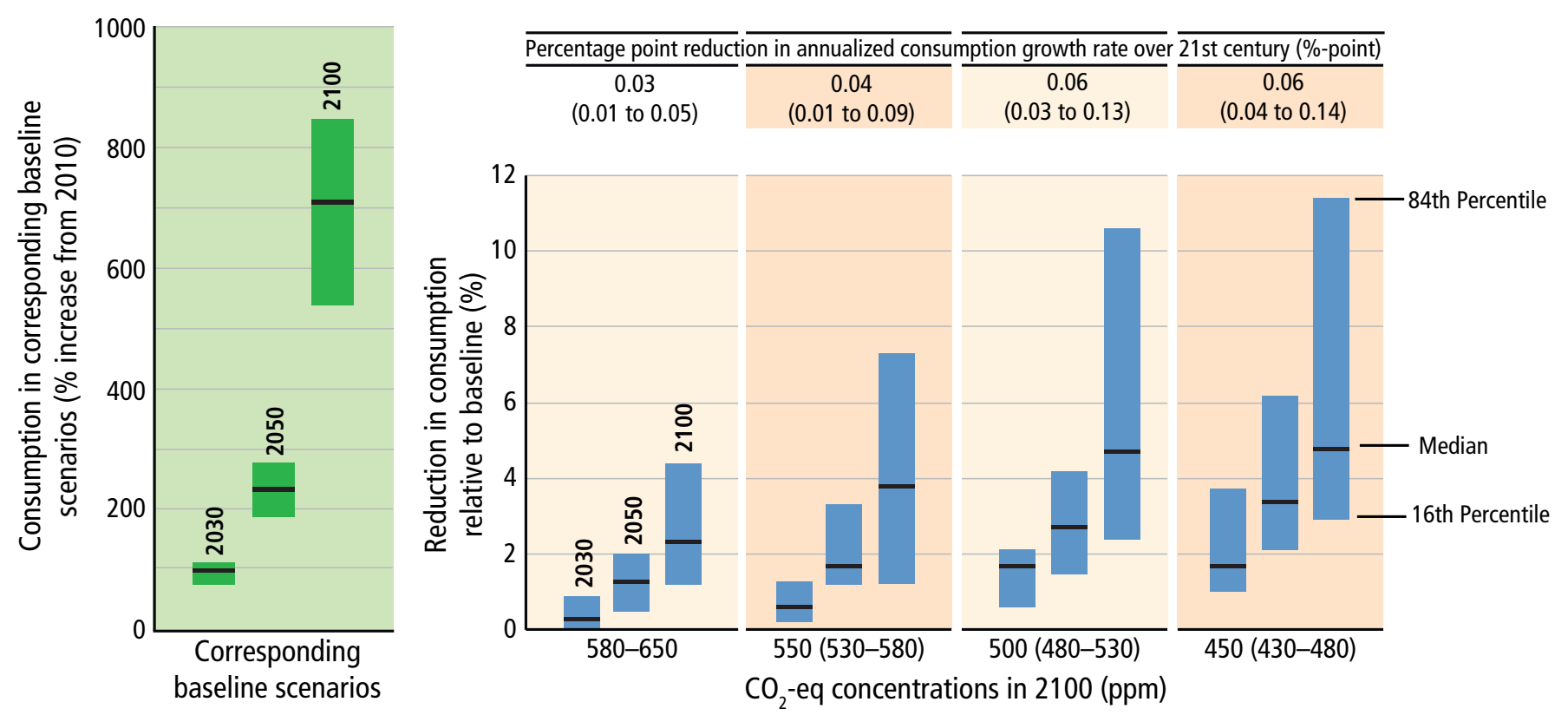

Figure 3.4 Global mitigation costs in cost-effective scenarios at different atmospheric concentrations levels in 2100 (right panel) and growth in economic consumption in the corresponding baseline scenarios (those without additional mitigation) (left panel). The table at the top shows percentage points of annualized consumption growth reductions relative to consumption growth in the baseline of 1.6 to $3 \%$ per year (e.g., if the reduction is 0.06 percentage points per year due to mitigation, and baseline growth is $2.0 \%$ per year, then the growth rate with mitigation would be $1.94 \%$ per year). Cost-effective scenarios assume immediate mitigation in all countries and a single global carbon price, and they impose no additional limitations on technology relative to the models' default technology assumptions. Consumption losses are shown relative to a baseline development without climate policy. Cost estimates shown in this table do not consider the benefits of reduced climate change nor co-benefits and adverse side effects of mitigation. Estimates at the high end of these cost ranges are from models that are relatively inflexible to achieve the deep emissions reductions that would be required in the long run to meet these goals and/or include assumptions about market imperfections that would raise costs. \{WGIII Table SPM.2, Figure TS. 12, 6.3.6, Figure 6.21\}

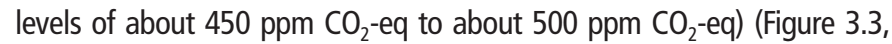
left panel). Scenarios with $\mathrm{GHG}$ emission levels of above $55 \mathrm{GtCO}_{2}$-eq/yr require substantially higher rates of emissions reductions between 2030 and 2050 (median estimate of 6\%/yr as compared to 3\%/yr in cost-effective scenarios; Figure 3.3, middle panel); much more rapid scale-up of zero and low-carbon energy over this period (more than a tripling compared to a doubling of the low-carbon energy share relative to 2010; Figure 3.3, right panel); a larger reliance on CDR technologies in the long term; and higher transitional and long-term economic impacts (Table 3.2). (3.5, 4.3) \{WGIIISPM.4.1, TS.3.1, 6.4, 7.11\}

Estimated global emission levels by 2020 based on the Cancún Pledges are not consistent with cost-effective long-term mitigation trajectories that are at least about as likely as not to limit warming to below $2^{\circ} \mathrm{C}$ relative to pre-industrial levels $(2100$ concentration levels of about $500 \mathrm{ppm} \mathrm{CO}_{2}$-eq or below), but they do not preclude the option to meet this goal (high confidence). The Cancún Pledges are broadly consistent with cost-effective scenarios that are likely to limit temperature change to below $3^{\circ} \mathrm{C}$ relative to pre-industrial levels. \{WGIII SPM.4.1, 6.4, 13.13, Figure TS.11\}

Estimates of the aggregate economic costs of mitigation vary widely depending on methodologies and assumptions but increase with the stringency of mitigation (high confidence). Scenarios in which all countries of the world begin mitigation immediately, in which there is a single global carbon price, and in which all key technologies are available have been used as a cost-effective benchmark for estimating macroeconomic mitigation costs (Figure 3.4). Under these assumptions, mitigation scenarios that are likely to limit warming to below $2^{\circ} \mathrm{C}$ through the 21 st century relative to pre-industrial levels entail losses in global consumption-not including benefits of reduced climate change (3.2) as well as co-benefits and adverse side effects of mitigation $(3.5,4.3$ ) - of 1 to $4 \%$ (median: 1.7\%) in 2030, 2 to $6 \%$ (median: $3.4 \%$ ) in 2050 , and $3 \%$ to $11 \%$ (median: $4.8 \%$ ) in 2100 , relative to consumption in baseline scenarios that grows anywhere from $300 \%$ to more than $900 \%$ over the century ${ }^{41}$. These numbers correspond to an annualized reduction of consumption growth by 0.04 to 0.14 (median: 0.06 ) percentage points over the century relative to annualized consumption growth in the baseline that is between $1.6 \%$ and $3 \%$ per year (Figure 3.4). In the absence or under limited availability of mitigation technologies (such as bioenergy, CCS, and their combination BECCS, nuclear, wind and solar), mitigation costs can increase substantially depending on the technology considered (Table 3.2). Delaying additional mitigation reduces near-term costs but increases mitigation costs in the medium- to long-term (Table 3.2). Many models could not limit likely warming to below $2^{\circ} \mathrm{C}$ over the 21 st century relative to pre-industrial levels, if additional mitigation is considerably delayed, or if availability of key technologies, such as bioenergy, CCS and their combination (BECCS) are limited (high confidence) (Table 3.2). \{WGIII SPM.4.1, Table SPM.2, Table TS.2, TS.3.1, 6.3, 6.6\}

41 Mitigation cost ranges cited here refer to the 16th to 84th percentile of the underlying sample (see Figure 3.4). 
Mitigation efforts and associated cost are expected to vary across countries. The distribution of costs can differ from the distribution of the actions themselves (high confidence). In globally cost-effective scenarios, the majority of mitigation efforts takes place in countries with the highest future GHG emissions in baseline scenarios. Some studies exploring particular effort-sharing frameworks, under the assumption of a global carbon market, have estimated substantial global financial flows associated with mitigation in scenarios that are likely to more unlikely than likely to limit warming during the 21st century to less than $2^{\circ} \mathrm{C}$ relative to pre-industrial levels. \{WGIII SPM.4.1, TS.3.1, Box 3.5, 4.6, 6.3.6, Table 6.4, Figure 6.9, Figure 6.27, Figure 6.28, Figure 6.29, 13.4.2.4\}

Table 3.2 | Increase in global mitigation costs due to either limited availability of specific technologies or delays in additional mitigation a relative to cost-effective scenarios ${ }^{b}$. The increase in costs is given for the median estimate and the 16th to 84th percentile range of the scenarios (in parentheses). The sample size of each scenario set is provided in the coloured symbols ${ }^{c}$. The colours of the symbols indicate the fraction of models from systematic model comparison exercises that could successfully reach the targeted concentration level. \{WGIII Table SPM.2, Table TS.2, Figure TS.13, Figure 6.24, Figure 6.25\}

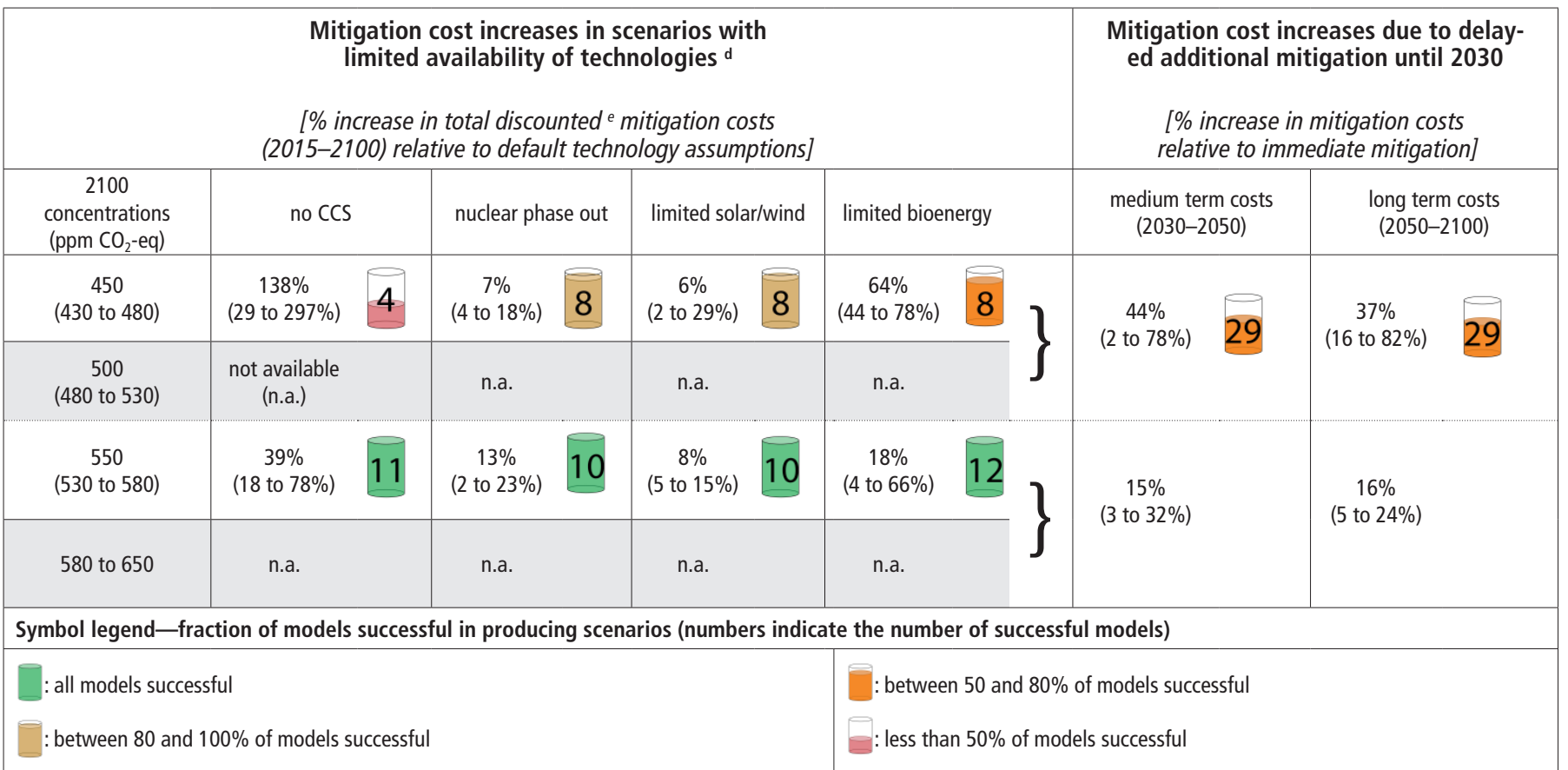

Notes:

a Delayed mitigation scenarios are associated with greenhouse gas emission of more than $55 \mathrm{GtCO}_{2}$-eq in 2030 , and the increase in mitigation costs is measured relative to costeffective mitigation scenarios for the same long-term concentration level.

b Cost-effective scenarios assume immediate mitigation in all countries and a single global carbon price, and impose no additional limitations on technology relative to the models' default technology assumptions.

c The range is determined by the central scenarios encompassing the 16th to 84th percentile range of the scenario set. Only scenarios with a time horizon until 2100 are included. Some models that are included in the cost ranges for concentration levels above $530 \mathrm{ppm} \mathrm{CO}_{2}$-eq in 2100 could not produce associated scenarios for concentration levels below 530 ppm CO

${ }^{d}$ No CCS: carbon dioxide capture and storage is not included in these scenarios. Nuclear phase out: no addition of nuclear power plants beyond those under construction, and operation of existing plants until the end of their lifetime. Limited Solar/Wind: a maximum of $20 \%$ global electricity generation from solar and wind power in any year of these scenarios. Limited Bioenergy: a maximum of $100 \mathrm{EJ} / \mathrm{yr}$ modern bioenergy supply globally (modern bioenergy used for heat, power, combinations and industry was around $18 \mathrm{EJ} / \mathrm{yr}$ in 2008). EJ = Exajoule $=10^{18}$ Joule.

e Percentage increase of net present value of consumption losses in percent of baseline consumption (for scenarios from general equilibrium models) and abatement costs in percent of baseline gross domestic product (GDP, for scenarios from partial equilibrium models) for the period 2015-2100, discounted at 5\% per year. 


\section{Box 3.2 | Greenhouse Gas Metrics and Mitigation Pathways}

This box focuses on emission-based metrics that are used for calculating $\mathrm{CO}_{2}$-equivalent emissions for the formulation and evaluation of mitigation strategies. These emission metrics are distinct from the concentration-based metric used in $\mathrm{SYR}\left(\mathrm{CO}_{2}\right.$-equivalent concentration). For an explanation of $\mathrm{CO}_{2}$-equivalent emissions and $\mathrm{CO}_{2}$-equivalent concentrations, see $\mathrm{Glossary}$.

Emission metrics facilitate multi-component climate policies by allowing emissions of different greenhouse gases (GHGs) and other climate forcing agents to be expressed in a common unit (so-called ' $\mathrm{CO}_{2}$-equivalent emissions'). The Global Warming Potential (GWP) was introduced in the IPCC First Assessment Report, where it was also used to illustrate the difficulties in comparing components with differing physical properties using a single metric. The 100-year GWP (GWP ${ }_{100}$ ) was adopted by the United Nations Framework Convention on Climate Change (UNFCCC) and its Kyoto Protocol and is now used widely as the default metric. It is only one of several possible emission metrics and time horizons. \{WGI 8.7, WGIII 3.9\}

The choice of emission metric and time horizon depends on type of application and policy context; hence, no single metric is optimal for all policy goals. All metrics have shortcomings, and choices contain value judgments, such as the climate effect considered and the weighting of effects over time (which explicitly or implicitly discounts impacts over time), the climate policy goal and the degree to which metrics incorporate economic or only physical considerations. There are significant uncertainties related to metrics, and the magnitudes of the uncertainties differ across metric type and time horizon. In general, the uncertainty increases for metrics along the cause-effect chain from emission to effects. \{WGI 8.7, WGIII 3.9\}

The weight assigned to non- $\mathrm{CO}_{2}$ climate forcing agents relative to $\mathrm{CO}_{2}$ depends strongly on the choice of metric and time horizon (robust evidence, high agreement). GWP compares components based on radiative forcing, integrated up to a chosen time horizon. Global Temperature change Potential (GTP; see Glossary) is based on the temperature response at a specific point in time with no weight on temperature response before or after the chosen point in time. Adoption of a fixed horizon of, for example, 20, 100 or 500 years for these metrics will inevitably put no weight on climate outcomes beyond the time horizon, which is significant for $\mathrm{CO}_{2}$ as well as other long-lived gases. The choice of time horizon markedly affects the weighting especially of short-lived climate forcing agents, such as methane $\left(\mathrm{CH}_{4}\right)$ (see Box 3.2, Table 1; Box 3.2, Figure 1a). For some metrics (e.g., the dynamic GTP; see Glossary), the weighting changes over time as a chosen target year is approached. \{WGI 8.7, WGIII 3.9\}

Box 3.2, Table 1 | Examples of emission metric values from WGI a.

\begin{tabular}{|l|c|c|c|c|c|}
\hline & Lifetime (yr) & $\begin{array}{c}\text { Cumulative forcing } \\
\text { over 20 years }\end{array}$ & $\begin{array}{c}\text { Cumulative forcing } \\
\text { over 100 years }\end{array}$ & $\begin{array}{c}\text { Temperature } \\
\text { change after 20 } \\
\text { years }\end{array}$ & $\begin{array}{c}\text { Temperature } \\
\text { change after 100 } \\
\text { years }\end{array}$ \\
\hline $\mathrm{CO}_{2}$ & $\mathrm{~b}$ & 1 & 1 & 1 & 1 \\
\hline $\mathrm{CH}_{4}$ & 12.4 & 84 & 28 & 67 & 4 \\
\hline $\mathrm{N}_{2} \mathrm{O}$ & 121.0 & 264 & 265 & 277 & 234 \\
\hline $\mathrm{CF}_{4}$ & $50,000.0$ & 4880 & 6630 & 5270 & 8040 \\
\hline $\mathrm{HFC}-152 \mathrm{a}$ & 1.5 & 506 & 138 & 174 & 19 \\
\hline
\end{tabular}

Notes:

a Global Warming Potential (GWP) values have been updated in successive IPCC reports; the AR5 GWP ${ }_{100}$ values are different from those adopted for the Kyoto Protocol's First Commitment Period which are from the IPCC Second Assessment Report (SAR). Note that for consistency, equivalent $\mathrm{CO}_{2}$ emissions given elsewhere in this Synthesis Report are also based on SAR, not AR5 values. For a comparison of emissions using SAR and AR5 GWP ${ }_{100}$ values for 2010 emissions, see Figure 1.6.

${ }^{\mathrm{b}}$ No single lifetime can be given for $\mathrm{CO}_{2}$. $\{W G I$ Box $6.1,6.1 .1,8.7\}$

The choice of emission metric affects the timing and emphasis placed on abating short- and long-lived climate forcing agents. For most metrics, global cost differences are small under scenarios of global participation and cost-minimizing mitigation pathways, but implications for some individual countries and sectors could be more significant (medium evidence, high agreement). Different metrics and time horizons significantly affect the contributions from various sources/sectors and components, particularly short-lived climate forcing agents (Box 3.2, Figure 1b). A fixed time independent metric that gives less weight to short-lived agents such as $\mathrm{CH}_{4}$ (e.g., using $\mathrm{GTP}_{100}$ instead of $\mathrm{GWP}_{100}$ ) would require earlier and more stringent $\mathrm{CO}_{2}$ abatement to achieve the same climate outcome for 2100 . Using a time-dependent metric, such as a dynamic GTP, leads to less $\mathrm{CH}_{4}$ mitigation 


\section{Box 3.2 (continued)}

in the near term but to more in the long term as the target date is being approached. This implies that for some (short-lived) agents, the metric choice influences the choice of policies and the timing of mitigation (especially for sectors and countries with high non- $\mathrm{CO}_{2}$ emission levels). \{WGI 8.7, WGIII 6.3\}

(a)

Weighting of current emissions over time
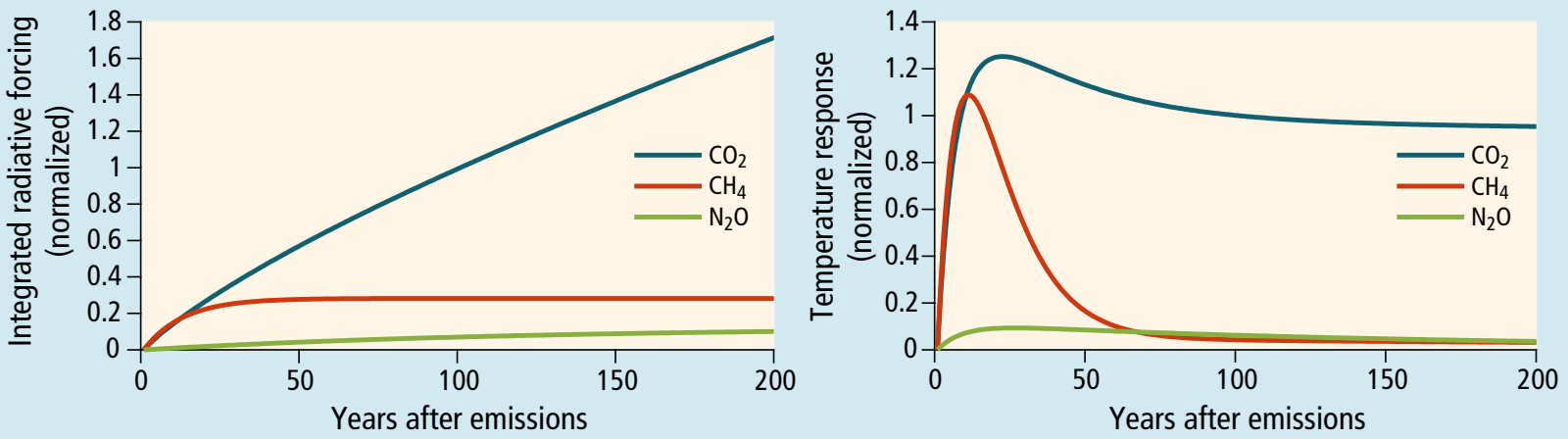

(b)

\section{Contributions by sectors to total GHG emissions using different metrics}
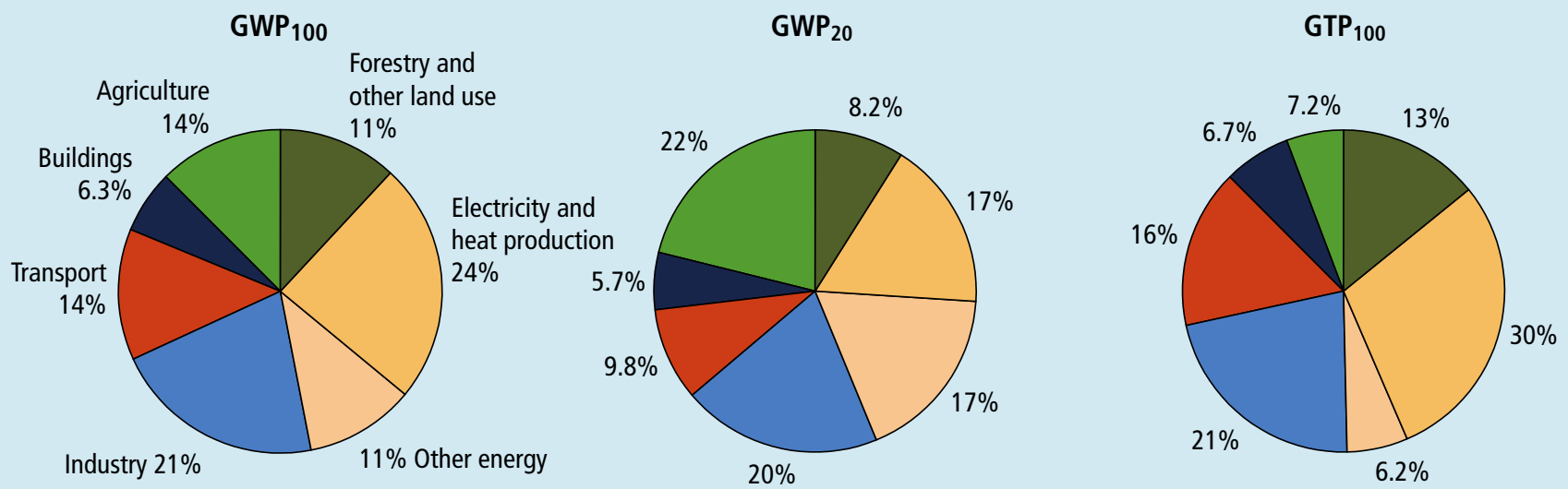

Box 3.2, Figure 1| Implications of metric choices on the weighting of greenhouse gas (GHG) emissions and contributions by sectors for illustrative time horizons. Panel (a): integrated radiative forcing (left panel) and warming resulting at a given future point in time (right panel) from global net emissions of carbon dioxide $\left(\mathrm{CO}_{2}\right)$, methane $\left(\mathrm{CH}_{4}\right)$ and nitrous oxide $\left(\mathrm{N}_{2} \mathrm{O}\right)$ in the year 2010 (and no emissions thereafter), for time horizons of up to 200 years. Integrated radiative forcing is used in the calculation of Global Warming Potentials (GWP), while the warming at a future point in time is used in the calculation of Global Temperature change Potentials (GTP). Radiative forcing and warming were calculated based on global 2010 emission data from WGIII 5.2 and absolute GWPs and absolute GTPs from WGI 8.7, normalized to the integrated radiative forcing and warming, respectively, after 100 years, due to 2010 net $\mathrm{CO}_{2}$ emissions. Panel (b): Illustrative examples showing contributions from different sectors to total metric-weighted global GHG emissions in the year 2010, calculated using 100-year GWP (GWP ${ }_{100}$, left), 20-year GWP (GWP 20 , middle) or 100-year GTP (GTP ${ }_{100}$, right) and the WGIII 2010 emissions database. \{WGIII 5.2\} Note that percentages differ slightly for the GWP ${ }_{100}$ case if values from the IPCC Second Assessment Report are used; see Topic 1, Figure 1.7. See WGIII for details of activities resulting in emissions in each sector. 


\section{Box 3.3 | Carbon Dioxide Removal and Solar Radiation Management Geoengineering Technologies- Possible Roles, Options, Risks and Status}

Geoengineering refers to a broad set of methods and technologies operating on a large scale that aim to deliberately alter the climate system in order to alleviate the impacts of climate change. Most methods seek to either reduce the amount of absorbed solar energy in the climate system (Solar Radiation Management, SRM) or increase the removal of carbon dioxide $\left(\mathrm{CO}_{2}\right)$ from the atmosphere by sinks to alter climate (Carbon Dioxide Removal, CDR, see Glossary). Limited evidence precludes a comprehensive assessment of feasibility, cost, side effects and environmental impacts of either CDR or SRM. \{WGI SPM E.8, 6.5, 7.7, WGII 6.4, Table 6-5, Box 20-4, WGIII TS.3.1.3, 6.9\}

CDR plays a major role in many mitigation scenarios. Bioenergy with carbon dioxide capture and storage (BECCS) and afforestation are the only CDR methods included in these scenarios. CDR technologies are particularly important in scenarios that temporarily overshoot atmospheric concentrations, but they are also prevalent in many scenarios without overshoot to compensate for residual emissions from sectors where mitigation is more expensive. Similar to mitigation, CDR would need to be deployed on a large scale and over a long time period to be able to significantly reduce $\mathrm{CO}_{2}$ concentrations (see Section 3.1). \{WGII 6.4, WGIII SPM 4.1, TS.3.1.2, TS 3.1.3, 6.3, 6.9\}

Several CDR techniques could potentially reduce atmospheric greenhouse gas (GHG) levels. However, there are biogeochemical, technical and societal limitations that, to varying degrees, make it difficult to provide quantitative estimates of the potential for CDR. The emission mitigation from CDR is less than the removed $\mathrm{CO}_{2}$, as some $\mathrm{CO}_{2}$ is released from that previously stored in oceans and terrestrial carbon reservoirs. Sub-sea geologic storage has been implemented on a regional scale, with no evidence to date of ocean impact from leakage. The climatic and environmental side effects of CDR depend on technology and scale. Examples are associated with altered surface reflectance from afforestation and ocean de-oxygenation from ocean fertilization. Most terrestrial CDR techniques would involve competing demands for land and could involve local and regional risks, while maritime CDR techniques may involve significant risks for ocean ecosystems, so that their deployment could pose additional challenges for cooperation between countries. \{WGI 6.5, FAQ 7.3, WGII 6.4, Table 6.5, WGIII 6.9\}

SRM is untested, and is not included in any of the mitigation scenarios, but, if realisable, could to some degree offset global temperature rise and some of its effects. It could possibly provide rapid cooling in comparison to $\mathrm{CO}_{2}$ mitigation. There is medium confidence that SRM through stratospheric aerosol injection is scalable to counter radiative forcing from a twofold increase in $\mathrm{CO}_{2}$ concentrations and some of the climate responses associated with warming. Due to insufficient understanding there is no consensus on whether a similarly large negative counter radiative forcing could be achieved from cloud brightening. Land albedo change does not appear to be able to produce a large counter radiative forcing. Even if SRM could counter the global mean warming, differences in spatial patterns would remain. The scarcity of literature on other SRM techniques precludes their assessment. \{WGI 7.7, WGIII TS.3.1.3, 6.9\}

If it were deployed, SRM would entail numerous uncertainties, side effects, risks and shortcomings. Several lines of evidence indicate that SRM would itself produce a small but significant decrease in global precipitation (with larger differences on regional scales). Stratospheric aerosol SRM is likely to modestly increase ozone losses in the polar stratosphere. SRM would not prevent the $\mathrm{CO}_{2}$ effects on ecosystems and ocean acidification that are unrelated to warming. There could also be other unanticipated consequences. For all future scenarios considered in AR5, SRM would need to increase commensurately, to counter the global mean warming, which would exacerbate side effects. Additionally, if SRM were increased to substantial levels and then terminated, there is high confidence that surface temperatures would rise very rapidly (within a decade or two). This would stress systems that are sensitive to the rate of warming. \{WGI 7.6-7.7, FAQ 7.3, WGII 19.5, WGIII 6.9\}

SRM technologies raise questions about costs, risks, governance and ethical implications of development and deployment. There are special challenges emerging for international institutions and mechanisms that could coordinate research and possibly restrain testing and deployment. Even if SRM would reduce human-made global temperature increase, it would imply spatial and temporal redistributions of risks. SRM thus introduces important questions of intragenerational and intergenerational justice. Research on SRM, as well as its eventual deployment, has been subject to ethical objections. In spite of the estimated low potential costs of some SRM deployment technologies, they will not necessarily pass a benefit-cost test that takes account of the range of risks and side effects. The governance implications of SRM are particularly challenging, especially as unilateral action might lead to significant effects and costs for others. \{WGIII TS.3.1.3, 1.4, 3.3, 6.9, 13.4\} 


\subsection{Interaction among mitigation, adaptation and sustainable development}

Climate change is a threat to equitable and sustainable development. Adaptation, mitigation and sustainable development are closely related, with potential for synergies and trade-offs.

Climate change poses an increasing threat to equitable and sustainable development (high confidence). Some climate-related impacts on development are already being observed. Climate change is a threat multiplier. It exacerbates other threats to social and natural systems, placing additional burdens particularly on the poor and constraining possible development paths for all. Development along current global pathways can contribute to climate risk and vulnerability, further eroding the basis for sustainable development. \{WGII SPM B-2, $2.5,10.9,13.1-13.3,20.1,20.2,20.6$, WGIII SPM.2, 4.2\}

Aligning climate policy with sustainable development requires attention to both adaptation and mitigation (high confidence). Interaction among adaptation, mitigation and sustainable development occurs both within and across regions and scales, often in the context of multiple stressors. Some options for responding to climate change could impose risks of other environmental and social costs, have adverse distributional effects and draw resources away from other development priorities, including poverty eradication. \{WGII 2.5, 8.4, 9.3, 13.3-13.4, 20.2-20.4, 21.4, 25.9, 26.8, WGIII SPM.2, 4.8, 6.6\}
Both adaptation and mitigation can bring substantial co-benefits (medium confidence). Examples of actions with co-benefits include (i) improved air quality (see Figure 3.5); (ii) enhanced energy security, (iii) reduced energy and water consumption in urban areas through greening cities and recycling water; (iv) sustainable agriculture and forestry; and (v) protection of ecosystems for carbon storage and other ecosystem services. \{WGII SPM C-1, WGIII SPM.4.1\}

Strategies and actions can be pursued now that will move towards climate-resilient pathways for sustainable development, while at the same time helping to improve livelihoods, social and economic well-being and effective environmental management (high confidence). Prospects for climate-resilient pathways are related fundamentally to what the world accomplishes with climate change mitigation (high confidence). Since mitigation reduces the rate as well as the magnitude of warming, it also increases the time available for adaptation to a particular level of climate change, potentially by several decades. Delaying mitigation actions may reduce options for climate-resilient pathways in the future. \{WGII SPM C-2, 20.2, 20.6.2\}
Co-benefits of climate change mitigation for air quality Impact of stringent climate policy on air pollutant emissions (Global, 2005-2050)

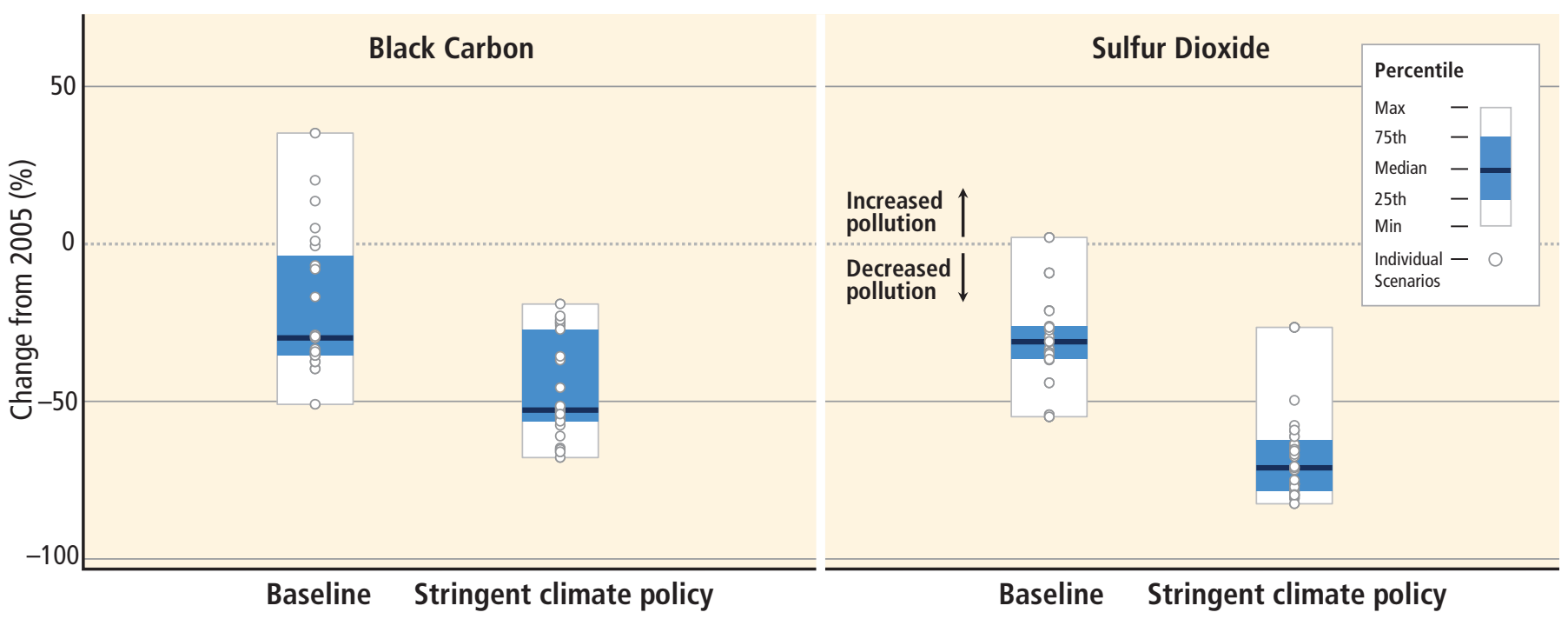

Figure 3.5 Air pollutant emission levels of black carbon (BC) and sulfur dioxide $\left(\mathrm{SO}_{2}\right)$ by 2050, relative to $2005(0=2005$ levels). Baseline scenarios without additional efforts to reduce greenhouse gas (GHG) emissions beyond those in place today are compared to scenarios with stringent mitigation policies, which are consistent with reaching about 450

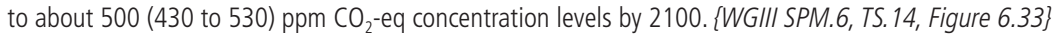




\section{Box 3.4 | Co-benefits and Adverse Side effects}

A government policy or a measure intended to achieve one objective often affects other objectives, either positively or negatively. For example, mitigation policies can influence local air quality (see Figure 3.5). When the effects are positive they are called 'co-benefits', also referred to as 'ancillary benefits'. Negative effects are referred to as 'adverse side effects'. Some measures are labelled 'no or low regret' when their co-benefits are sufficient to justify their implementation, even in the absence of immediate direct benefits. Co-benefits and adverse side effects can be measured in monetary or non-monetary units. The effect of co-benefits and adverse side effects from climate policies on overall social welfare has not yet been quantitatively examined, with the exception of a few recent multi-objective studies. Many of these have not been well quantified, and effects can be case and site-specific as they will depend on local circumstances. \{WGII 11.9, 16.3.1, 17.2, 20.4.1, WGIII Box TS.11, 3.6, 5.7\}

Co-benefits of mitigation could affect achievement of other objectives, such as those related to energy security, air quality, efforts to address ecosystem impacts, income distribution, labour supply and employment and urban sprawl (see Table 4.2 and Table 4.5). In the absence of complementary policies, however, some mitigation measures may have adverse side effects (at least in the short term), for example on biodiversity, food security, energy access, economic growth and income distribution. The co-benefits of adaptation policies may include improved access to infrastructure and services, extended education and health systems, reduced disaster losses, better governance and others. \{WGII 4.4.4, 11.9, 15.2, 17.2, 20.3.3, 20.4.1, WGIII Box TS.11, 6.6\}

Comprehensive strategies in response to climate change that are consistent with sustainable development take into account the co-benefits, adverse side effects and risks that may arise from both adaptation and mitigation options. The assessment of overall social welfare impacts is complicated by this interaction between climate change response options and preexisting non-climate policies. For example, in terms of air quality, the value of the extra tonne of sulfur dioxide $\left(\mathrm{SO}_{2}\right)$ reduction that occurs with climate change mitigation through reduced fossil fuel combustion depends greatly on the stringency of $\mathrm{SO}_{2}$ control policies. If $\mathrm{SO}_{2}$ policy is weak, the value of $\mathrm{SO}_{2}$ reductions may be large, but if $\mathrm{SO}_{2}$ policy is stringent, it may be near zero. Similarly, in terms of adaptation and disaster risk management, weak policies can lead to an adaptation deficit that increases human and economic losses from natural climate variability. 'Adaptation deficit' refers to the lack of capacity to manage adverse impacts of current climate variability. An existing adaptation deficit increases the benefits of adaptation policies that improve the management of climate variability and change. \{WGII 20.4.1, WGIII Box TS.11, 6.3\} 



\section{Adaptation and Mitigation}




\section{Topic 4: Adaptation and Mitigation}

Many adaptation and mitigation options can help address climate change, but no single option is sufficient by itself. Effective implementation depends on policies and cooperation at all scales and can be enhanced through integrated responses that link mitigation and adaptation with other societal objectives.

Topic 3 demonstrates the need and strategic considerations for both adaptation and global-scale mitigation to manage risks from climate change. Building on these insights, Topic 4 presents near-term response options that could help achieve such strategic goals. Near-term adaptation and mitigation actions will differ across sectors and regions, reflecting development status, response capacities and near- and long-term aspirations with regard to both climate and non-climate outcomes. Because adaptation and mitigation inevitably take place in the context of multiple objectives, particular attention is given to the ability to develop and implement integrated approaches that can build on co-benefits and manage trade-offs.

\subsection{Common enabling factors and constraints for adaptation and mitigation responses}

\begin{abstract}
Adaptation and mitigation responses are underpinned by common enabling factors. These include effective institutions and governance, innovation and investments in environmentally sound technologies and infrastructure, sustainable livelihoods and behavioural and lifestyle choices.
\end{abstract}

Innovation and investments in environmentally sound infrastructure and technologies can reduce greenhouse gas (GHG) emissions and enhance resilience to climate change (very high confidence). Innovation and change can expand the availability and/ or effectiveness of adaptation and mitigation options. For example, investments in low-carbon and carbon-neutral energy technologies can reduce the energy intensity of economic development, the carbon intensity of energy, GHG emissions, and the long-term costs of mitigation. Similarly, new technologies and infrastructure can increase the resilience of human systems while reducing adverse impacts on natural systems. Investments in technology and infrastructure rely on an enabling policy environment, access to finance and technology and broader economic development that builds capacity (Table 4.1, Section 4.4). \{WGII SPM C-2, Table SPM.1, Table TS.8, WGIII SPM.4.1, Table SPM.2, TS.3.1.1, TS 3.1.2, TS.3.2.1\}

Adaptation and mitigation are constrained by the inertia of global and regional trends in economic development, GHG emissions, resource consumption, infrastructure and settlement patterns, institutional behaviour and technology (medium evidence, high agreement). Such inertia may limit the capacity to reduce GHG emissions, remain below particular climate thresholds or avoid adverse impacts (Table 4.1). Some constraints may be overcome through new technologies, financial resources, increased institutional effectiveness and governance or changes in social and cultural attitudes and behaviours. \{WGII SPM C-1, WGIII SPM.3, SPM.4.2, Table SPM.2\}

Vulnerability to climate change, GHG emissions, and the capacity for adaptation and mitigation are strongly influenced by livelihoods, lifestyles, behaviour and culture (medium evidence, medium agreement) (Table 4.1). Shifts toward more energy-intensive lifestyles can contribute to higher energy and resource consumption, driving greater energy production and GHG emissions and increasing mitigation costs. In contrast, emissions can be substantially lowered through changes in consumption patterns (see 4.3 for details). The social acceptability and/or effectiveness of climate policies are influenced by the extent to which they incentivize or depend on regionally appropriate changes in lifestyles or behaviours. Similarly, livelihoods that depend on climate-sensitive sectors or resources may be particularly vulnerable to climate change and climate change policies. Economic development and urbanization of landscapes exposed to climate hazards may increase the exposure of human settlements and reduce the resilience of natural systems. \{WGII SPM A-2, SPM B-2, Table SPM.1, TS A-1, TS A-2, TS C-1, TS C-2, 16.3.2.7, WGIII SPM.4.2, TS.2.2, 4.2\}

For many regions and sectors, enhanced capacities to mitigate and adapt are part of the foundation essential for managing climate change risks (high confidence). Such capacities are place- and context-specific and therefore there is no single approach for reducing risk that is appropriate across all settings. For example, developing nations with low income levels have the lowest financial, technological and institutional capacities to pursue low-carbon, climate-resilient development pathways. Although developed nations generally have greater relative capacity to manage the risks of climate change, such capacity does not necessarily translate into the implementation of adaptation and mitigation options. \{WGII SPM B-1, SPM B-2, TS B-1, TS B-2, 16.3.1.1, 16.3.2, 16.5, WGIII SPM.5.1, TS.4.3, TS.4.5, 4.6\}

Improving institutions as well as enhancing coordination and cooperation in governance can help overcome regional constraints associated with mitigation, adaptation and disaster risk reduction (very high confidence). Despite the presence of a wide array of multilateral, national and sub-national institutions focused on adaptation and mitigation, global GHG emissions continue to increase and identified adaptation needs have not been adequately addressed. The implementation of effective adaptation and mitigation options may necessitate new institutions and institutional arrangements that span multiple scales (medium confidence) (Table 4.1). \{WGII SPM B-2, TS C-1, 16.3.2.4, 16.8, WGIII SPM.4.2.5, SPM.5.1, SPM.5.2, TS.1, TS.3.1.3, TS.4.1, TS.4.2, TS.4.4\} 
Table 4.1 | Common factors that constrain the implementation of adaptation and mitigation options

\begin{tabular}{|c|c|c|}
\hline Constraining Factor & Potential Implications for Adaptation & Potential Implications for Mitigation \\
\hline $\begin{array}{l}\text { Adverse externalities of popula- } \\
\text { tion growth and urbanization }\end{array}$ & $\begin{array}{l}\text { Increase exposure of human populations to climate variability } \\
\text { and change as well as demands for, and pressures on, natural } \\
\text { resources and ecosystem services }\{W G I I 16.3 .2 .3, \text { Box 16-3\} }\end{array}$ & $\begin{array}{l}\text { Drive economic growth, energy demand and energy consumption, } \\
\text { resulting in increases in greenhouse gas emissions \{WGIII SPM. } 3\}\end{array}$ \\
\hline $\begin{array}{l}\text { Deficits of knowledge, edu- } \\
\text { cation and human capital }\end{array}$ & $\begin{array}{l}\text { Reduce national, institutional and individual perceptions of } \\
\text { the risks posed by climate change as well as the costs and } \\
\text { benefits of different adaptation options }\{W G I I 16.3 .2 .1\}\end{array}$ & $\begin{array}{l}\text { Reduce national, institutional and individual risk perception, } \\
\text { willingness to change behavioural patterns and practices and to } \\
\text { adopt social and technological innovations to reduce emissions } \\
\text { \{WGIII SPM.3, SPM.5.1, 2.4.1, 3.10.1.5, 4.3.5, 9.8, 11.8.1\} }\end{array}$ \\
\hline $\begin{array}{l}\text { Divergences in social and cultural } \\
\text { attitudes, values and behaviours }\end{array}$ & $\begin{array}{l}\text { Reduce societal consensus regarding climate risk and therefore } \\
\text { demand for specific adaptation policies and measures \{WGII } \\
\text { 16.3.2.7\} }\end{array}$ & $\begin{array}{l}\text { Influence emission patterns, societal perceptions of the } \\
\text { utility of mitigation policies and technologies, and willing- } \\
\text { ness to pursue sustainable behaviours and technologies } \\
\text { \{WGIII SPM.2, 2.4.5, 2.6.6.1, 3.7.2.2, 3.9.2, 4.3.4, 5.5.1\} }\end{array}$ \\
\hline $\begin{array}{l}\text { Challenges in governance and } \\
\text { institutional arrangements }\end{array}$ & $\begin{array}{l}\text { Reduce the ability to coordinate adaptation policies and } \\
\text { measures and to deliver capacity to actors to plan and implement } \\
\text { adaptation }\{W G / l \text { 16.3.2.8\} }\end{array}$ & $\begin{array}{l}\text { Undermine policies, incentives and cooperation regarding the } \\
\text { development of mitigation policies and the implementation of } \\
\text { efficient, carbon-neutral and renewable energy technologies } \\
\text { \{WGIII SPM.3, SPM.5.2, 4.3.2, 6.4.3, 14.1.3.1, 14.3.2.2, 15.12.2, } \\
\text { 16.5.3\} }\end{array}$ \\
\hline $\begin{array}{l}\text { Lack of access to national and } \\
\text { international climate finance }\end{array}$ & $\begin{array}{l}\text { Reduces the scale of investment in adaptation policies and } \\
\text { measures and therefore their effectiveness }\{W G I I \text { 16.3.2.5\} }\end{array}$ & $\begin{array}{l}\text { Reduces the capacity of developed and, particularly, developing } \\
\text { nations to pursue policies and technologies that reduce emissi- } \\
\text { ons. }\{\text { WGIII TS.4.3, 12.6.2, 16.2.2.2\} }\end{array}$ \\
\hline Inadequate technology & $\begin{array}{l}\text { Reduces the range of available adaptation options as well as } \\
\text { their effectiveness in reducing or avoiding risk from increasing } \\
\text { rates or magnitudes of climate change }\{W G / l \text { 16.3.2.1\} }\end{array}$ & $\begin{array}{l}\text { Slows the rate at which society can reduce the carbon intensity of } \\
\text { energy services and transition toward low-carbon and carbon-neutral } \\
\text { technologies }\{W G I I I T S .3 .1 .3,4.3 .6,6.3 .2 .2,11.8 .4\}\end{array}$ \\
\hline $\begin{array}{l}\text { Insufficient quality and/or quan- } \\
\text { tity of natural resources }\end{array}$ & $\begin{array}{l}\text { Reduce the coping range of actors, vulnerability to non-climatic } \\
\text { factors and potential competition for resources that enhances } \\
\text { vulnerability \{WGII 16.3.2.3\} }\end{array}$ & $\begin{array}{l}\text { Reduce the long-term sustainability of different energy } \\
\text { technologies }\{W G \text { III } 4.3 .7,4.4 .1,11.8 .3\}\end{array}$ \\
\hline Adaptation and development deficits & $\begin{array}{l}\text { Increase vulnerability to current climate variability as well as } \\
\text { future climate change }\{W G I I T S A-1 \text {, Table TS 5, 16.3.2.4\} }\end{array}$ & $\begin{array}{l}\text { Reduce mitigative capacity and undermine international } \\
\text { cooperative efforts on climate owing to a contentious legacy } \\
\text { of cooperation on development }\{W G I I I 4.3 .1,4.6 .1\}\end{array}$ \\
\hline Inequality & $\begin{array}{l}\text { Places the impacts of climate change and the burden of adapta- } \\
\text { tion disproportionately on the most vulnerable and/or transfers } \\
\text { them to future generations \{WGII TS B-2, Box TS 4, Box 13-1, } \\
\text { 16.7\} }\end{array}$ & $\begin{array}{l}\text { Constrains the ability for developing nations with low income } \\
\text { levels, or different communities or sectors within nations, to } \\
\text { contribute to greenhouse gas mitigation }\{W G I I I 4.6 .2 .1\}\end{array}$ \\
\hline
\end{tabular}

\subsection{Response options for adaptation}

Adaptation options exist in all sectors, but their context for implementation and potential to reduce climate-related risks differs across sectors and regions. Some adaptation responses involve significant co-benefits, synergies and trade-offs. Increasing climate change will increase challenges for many adaptation options.

People, governments and the private sector are starting to adapt to a changing climate. Since the IPCC Fourth Assessment Report (AR4), understanding of response options has increased, with improved knowledge of their benefits, costs and links to sustainable development. Adaptation can take a variety of approaches depending on its context in vulnerability reduction, disaster risk management or proactive adaptation planning. These include (see Table 4.2 for examples and details):
- Social, ecological asset and infrastructure development

- Technological process optimization

- Integrated natural resources management

- Institutional, educational and behavioural change or reinforcement

- Financial services, including risk transfer

- Information systems to support early warning and proactive planning

There is increasing recognition of the value of social (including local and indigenous), institutional, and ecosystem-based measures and of the extent of constraints to adaptation. Effective strategies and actions consider the potential for co-benefits and opportunities within wider strategic goals and development plans. \{WGII SPMA-2, SPM C-1, TS A-2, 6.4, 8.3, 9.4, 15.3\}

Opportunities to enable adaptation planning and implementation exist in all sectors and regions, with diverse potential and approaches depending on context. The need for adaptation along with associated challenges is expected to increase with climate change (very high confidence). Examples of key adaptation approaches for particular sectors, including constraints and limits, are summarized below. \{WGII SPM B, SPM C, 16.4, 16.6, 17.2, 19.6, 19.7, Table 16.3\} 
Table 4.2 |Approaches for managing the risks of climate change through adaptation. These approaches should be considered overlapping rather than discrete, and they are often pursued simultaneously. Examples are presented in no specific order and can be relevant to more than one category. \{WGII Table SPM.1\}

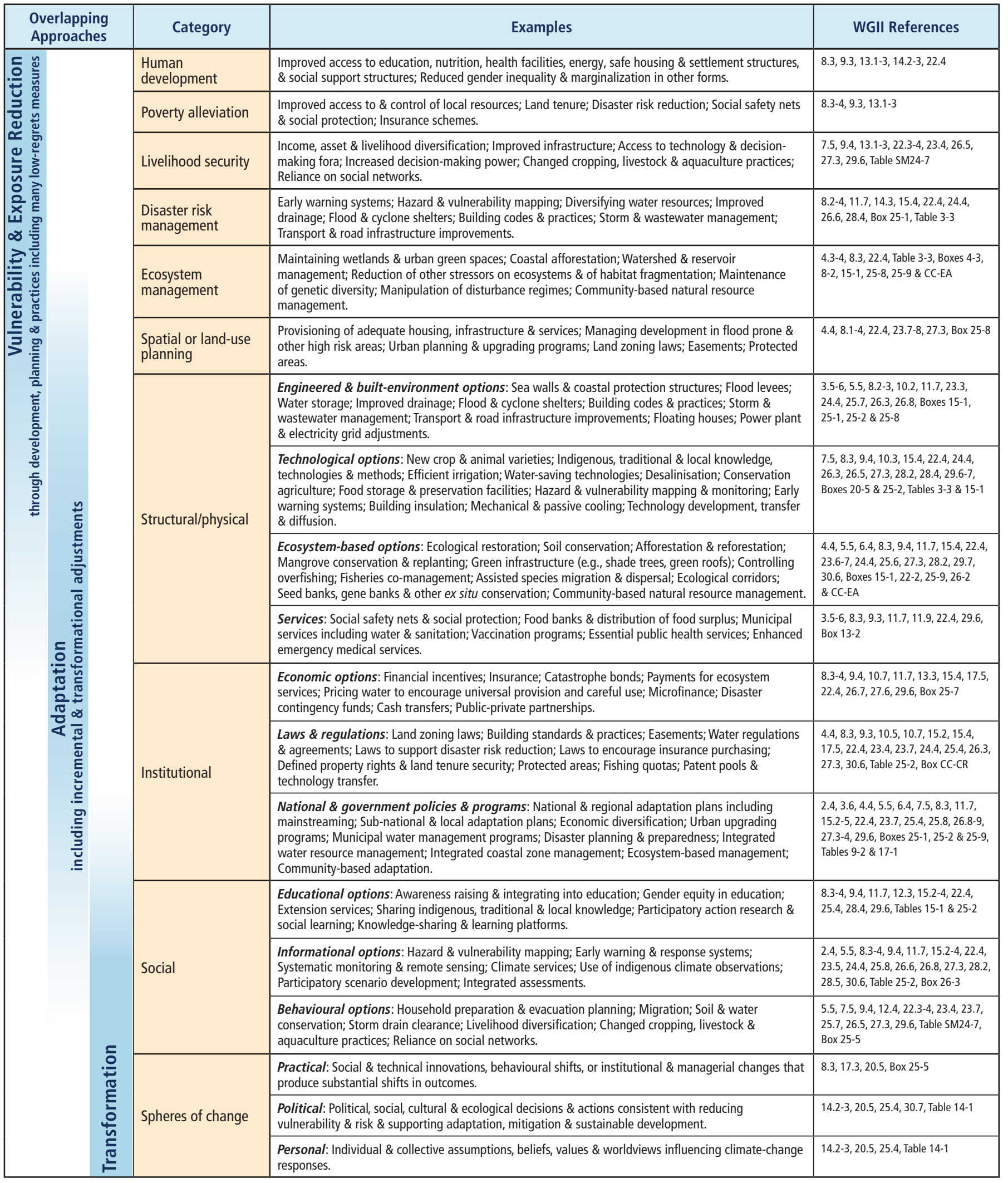




\section{Freshwater resources}

Adaptive water management techniques, including scenario planning, learning-based approaches and flexible and low-regret solutions, can help adjust to uncertain hydrological changes due to climate change and their impacts (limited evidence, high agreement). Strategies include adopting integrated water management, augmenting supply, reducing the mismatch between water supply and demand, reducing non-climate stressors, strengthening institutional capacities and adopting more water-efficient technologies and water-saving strategies. \{WGII SPM B-2, Assessment Box SPM.2 Table 1, SPM B-3, 3.6, 22.3-22.4, 23.4, 23.7, 24.4, 27.2-27.3, Box 25-2\}

\section{Terrestrial and freshwater ecosystems}

Management actions can reduce but not eliminate risks of impacts to terrestrial and freshwater ecosystems due to climate change (high confidence). Actions include maintenance of genetic diversity, assisted species migration and dispersal, manipulation of disturbance regimes (e.g., fires, floods) and reduction of other stressors. Management options that reduce non-climatic stressors, such as habitat modification, overexploitation, pollution and invasive species, increase the inherent capacity of ecosystems and their species to adapt to a changing climate. Other options include improving early warning systems and associated response systems. Enhanced connectivity of vulnerable ecosystems may also assist autonomous adaptation. Translocation of species is controversial and is expected to become less feasible where whole ecosystems are at risk. \{WGII SPM $B-2$, SPM B-3, Figure SPM.5, Table TS.8, 4.4, 25.6, 26.4, Box CC-RF\}

\section{Coastal systems and low-lying areas}

Increasingly, coastal adaptation options include those based on integrated coastal zone management, local community participation, ecosystems-based approaches and disaster risk reduction, mainstreamed into relevant strategies and management plans (high confidence). The analysis and implementation of coastal adaptation has progressed more significantly in developed countries than in developing countries (high confidence). The relative costs of coastal adaptation are expected to vary strongly among and within regions and countries. \{WGII SPM B-2, SPM B-3, 5.5, 8.3, 22.3, 24.4, 26.8, Box 25-1\}

\section{Marine systems and oceans}

Marine forecasting and early warning systems as well as reducing non-climatic stressors have the potential to reduce risks for some fisheries and aquaculture industries, but options for unique ecosystems such as coral reefs are limited (high confidence). Fisheries and some aquaculture industries with high-technology and/or large investments have high capacities for adaptation due to greater development of environmental monitoring, modelling and resource assessments. Adaptation options include large-scale translocation of industrial fishing activities and flexible management that can react to variability and change. For smaller-scale fisheries and nations with limited adaptive capacities, building social resilience, alternative livelihoods and occupational flexibility are important strategies. Adaptation options for coral reef systems are generally limited to reducing other stressors, mainly by enhancing water quality and limiting pressures from tourism and fishing, but their efficacy will be severely reduced as thermal stress and ocean acidification increase. \{WGII SPM B-2, SPM Assessment Box SPM.2 Table 1, TS B-2, 5.5, 6.4, 7.5, 25.6.2, 29.4, 30.6-7, Box CC-MB, Box CC-CR\}

\section{Food production system/Rural areas}

Adaptation options for agriculture include technological responses, enhancing smallholder access to credit and other critical production resources, strengthening institutions at local to regional levels and improving market access through trade reform (medium confidence). Responses to decreased food production and quality include: developing new crop varieties adapted to changes in $\mathrm{CO}_{2}$, temperature, and drought; enhancing the capacity for climate risk management; and offsetting economic impacts of land use change. Improving financial support and investing in the production of small-scale farms can also provide benefits. Expanding agricultural markets and improving the predictability and reliability of the world trading system could result in reduced market volatility and help manage food supply shortages caused by climate change. [WGII SPM $B-2$, SPM B-3, 7.5, 9.3, 22.4, 22.6, 25.9, 27.3\}

\section{Urban areas/Key economic sectors and services}

Urban adaptation benefits from effective multi-level governance, alignment of policies and incentives, strengthened local government and community adaptation capacity, synergies with the private sector and appropriate financing and institutional development (medium confidence). Enhancing the capacity of low-income groups and vulnerable communities and their partnerships with local governments can also be an effective urban climate adaptation strategy. Examples of adaptation mechanisms include large-scale public-private risk reduction initiatives and economic diversification and government insurance for the non-diversifiable portion of risk. In some locations, especially at the upper end of projected climate changes, responses could also require transformational changes such as managed retreat. \{WGII SPM B-2, 8.3-8.4, 24.4, 24.5, 26.8, Box 25-9\}

\section{Human health, security and livelihoods}

Adaptation options that focus on strengthening existing delivery systems and institutions, as well as insurance and social protection strategies, can improve health, security and livelihoods in the near term (high confidence). The most effective vulnerability reduction measures for health in the near term are programmes that implement and improve basic public health measures such as provision of clean water and sanitation, secure essential health care including vaccination and child health services, increase capacity for disaster preparedness and response and alleviate poverty (very high confidence). Options to address heat related mortality include health warning systems linked to response strategies, urban planning and improvements to the built environment to reduce heat stress. Robust institutions can manage many transboundary impacts of climate change to reduce risk of conflicts over shared natural resources. Insurance programmes, social protection measures and disaster risk management may enhance long-term livelihood resilience among the poor and marginalized people, if policies address multi-dimensional poverty. \{WGII SPM $B-2$, SPM B-3, 8.2, 10.8, 11.7-11.8, 12.5-12.6, 22.3, 23.9, 25.8, 26.6, Box CC-HS\} 
Table 4.3 | Examples of potential trade-offs associated with an illustrative set of adaptation options that could be implemented by actors to achieve specific management objectives. $\{W G / l$ Table 16-2\}

\begin{tabular}{|c|c|c|c|}
\hline Sector & Actor's adaptation objective & Adaptation option & Real or perceived trade-off \\
\hline \multirow[t]{3}{*}{ Agriculture } & Enhance drought and pest resistance; enhance yields & $\begin{array}{l}\text { Biotechnology and } \\
\text { genetically modified crops }\end{array}$ & $\begin{array}{l}\text { Perceived risk to public health and safety; } \\
\text { ecological risks associated with introduction of } \\
\text { new genetic variants to natural environments }\end{array}$ \\
\hline & $\begin{array}{l}\text { Provide financial safety net for farmers to } \\
\text { ensure continuation of farming enterprises }\end{array}$ & $\begin{array}{l}\text { Subsidized drought } \\
\text { assistance; crop insurance }\end{array}$ & $\begin{array}{l}\text { Creates moral hazard and distributional } \\
\text { inequalities if not appropriately administered }\end{array}$ \\
\hline & $\begin{array}{l}\text { Maintain or enhance crop yields; suppress } \\
\text { opportunistic agricultural pests and invasive species }\end{array}$ & $\begin{array}{l}\text { Increased use of chemical } \\
\text { fertilizer and pesticides }\end{array}$ & $\begin{array}{l}\text { Increased discharge of nutrients and chemical pollution } \\
\text { to the environment; adverse impacts of pesticide use on } \\
\text { non-target species; increased emissions of greenhouse } \\
\text { gases; increased human exposure to pollutants }\end{array}$ \\
\hline \multirow[t]{2}{*}{ Biodiversity } & $\begin{array}{l}\text { Enhance capacity for natural adaptation and } \\
\text { migration to changing climatic conditions }\end{array}$ & $\begin{array}{l}\text { Migration corridors; } \\
\text { expansion of } \\
\text { conservation areas }\end{array}$ & $\begin{array}{l}\text { Unknown efficacy; concerns over property rights } \\
\text { regarding land acquisition; governance challenges }\end{array}$ \\
\hline & $\begin{array}{l}\text { Enhance regulatory protections for species potentially } \\
\text { at risk due to climate and non-climatic changes }\end{array}$ & $\begin{array}{l}\text { Protection of critical habitat } \\
\text { for vulnerable species }\end{array}$ & $\begin{array}{l}\text { Addresses secondary rather than primary pressures } \\
\text { on species; concerns over property rights; regulatory } \\
\text { barriers to regional economic development }\end{array}$ \\
\hline \multirow[t]{3}{*}{ Coasts } & $\begin{array}{l}\text { Provide near-term protection to financial } \\
\text { assets from inundation and/or erosion }\end{array}$ & Sea walls & $\begin{array}{l}\text { High direct and opportunity costs; equity concerns; } \\
\text { ecological impacts to coastal wetlands }\end{array}$ \\
\hline & $\begin{array}{l}\text { Allow natural coastal and ecological processes to } \\
\text { proceed; reduce long-term risk to property and assets }\end{array}$ & Managed retreat & $\begin{array}{l}\text { Undermines private property rights; significant governance } \\
\text { challenges associated with implementation }\end{array}$ \\
\hline & $\begin{array}{l}\text { Preserve public health and safety; minimize } \\
\text { property damage and risk of stranded assets }\end{array}$ & $\begin{array}{l}\text { Migration out of } \\
\text { low-lying areas }\end{array}$ & $\begin{array}{l}\text { Loss of sense of place and cultural identity; erosion of } \\
\text { kinship and familial ties; impacts to receiving communities }\end{array}$ \\
\hline \multirow[t]{2}{*}{$\begin{array}{l}\text { Water resources } \\
\text { management }\end{array}$} & $\begin{array}{l}\text { Increase water resource reliability } \\
\text { and drought resilience }\end{array}$ & Desalination & $\begin{array}{l}\text { Ecological risk of saline discharge; high energy } \\
\text { demand and associated carbon emissions; } \\
\text { creates disincentives for conservation }\end{array}$ \\
\hline & $\begin{array}{l}\text { Maximize efficiency of water management } \\
\text { and use; increase flexibility }\end{array}$ & Water trading & Undermines public good/social aspects of water \\
\hline
\end{tabular}

Significant co-benefits, synergies and trade-offs exist between adaptation and mitigation and among different adaptation responses; interactions occur both within and across regions and sectors (very high confidence). For example, investments in crop varieties adapted to climate change can increase the capacity to cope with drought, and public health measures to address vector-borne diseases can enhance the capacity of health systems to address other challenges. Similarly, locating infrastructure away from low-lying coastal areas helps settlements and ecosystems adapt to sea level rise while also protecting against tsunamis. However, some adaptation options may have adverse side effects that imply real or perceived trade-offs with other adaptation objectives (see Table 4.3 for examples), mitigation objectives or broader development goals. For example, while protection of ecosystems can assist adaptation to climate change and enhance carbon storage, increased use of air conditioning to maintain thermal comfort in buildings or the use of desalination to enhance water resource security can increase energy demand, and therefore, GHG emissions. \{WGII SPM B-2, SPM C-1, 5.4.2, 16.3.2.9, 17.2.3.1, Table 16-2\}

\subsection{Response options for mitigation}

Mitigation options are available in every major sector. Mitigation can be more cost-effective if using an integrated approach that combines measures to reduce energy use and the greenhouse gas intensity of end-use sectors, decarbonize energy supply, reduce net emissions and enhance carbon sinks in land-based sectors.

A broad range of sectoral mitigation options is available that can reduce GHG emission intensity, improve energy intensity through enhancements of technology, behaviour, production and resource efficiency and enable structural changes or changes in activity. In addition, direct options in agriculture, forestry and other land use (AFOLU) involve reducing $\mathrm{CO}_{2}$ emissions by reducing deforestation, forest degradation and forest fires; storing carbon in terrestrial systems (for example, through afforestation); and providing bioenergy feedstocks. Options to reduce non- $\mathrm{CO}_{2}$ emissions exist across all sectors but most notably in agriculture, energy supply and 


\section{Sectoral $\mathrm{CO}_{2}$ and non- $\mathrm{CO}_{2}$ GHG emissions in baseline and mitigation scenarios with and without CCS}
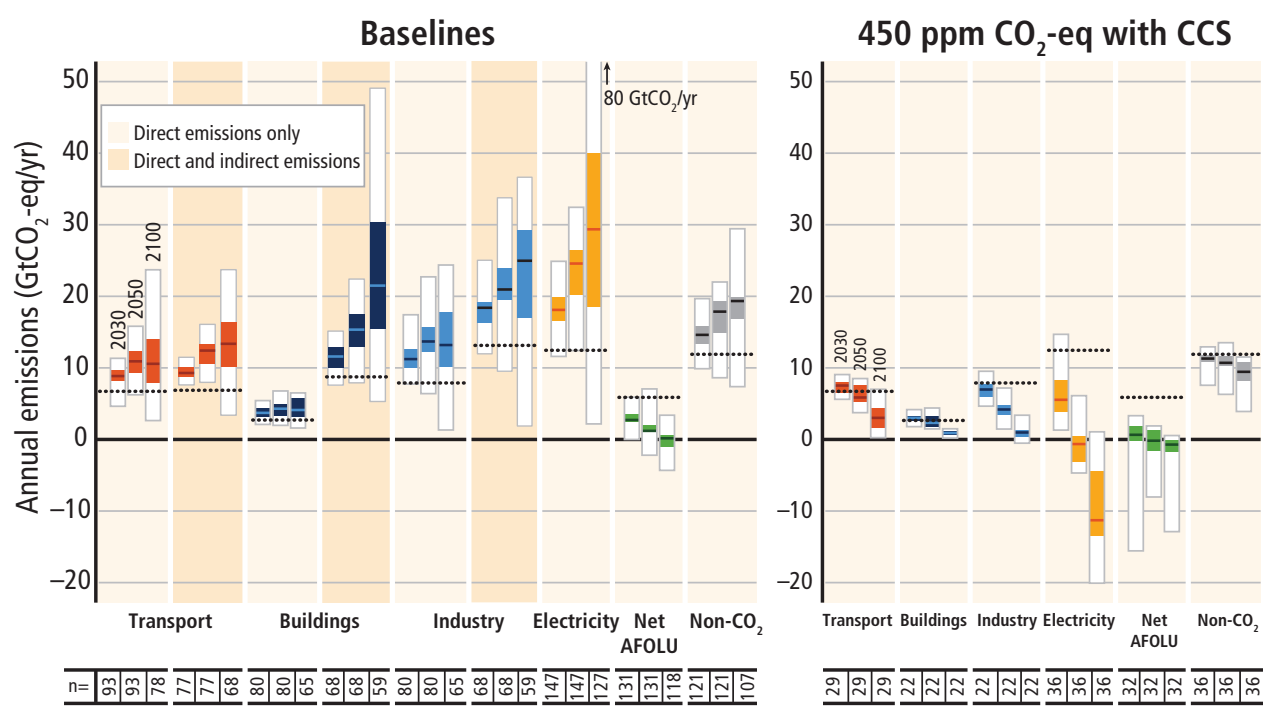
$450 \mathrm{ppm} \mathrm{CO}$-eq without CCS

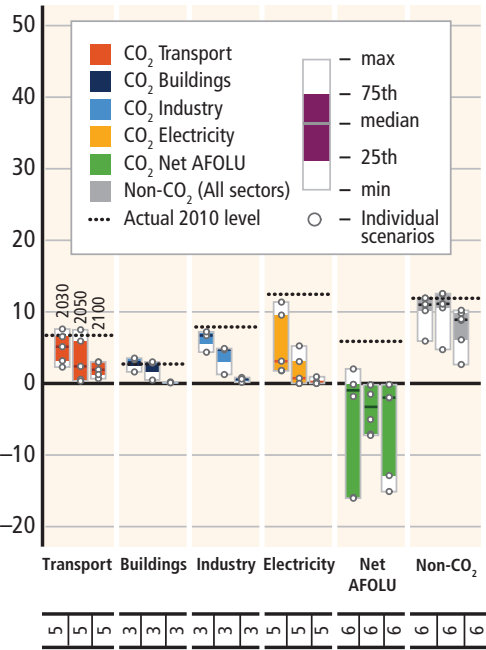

Figure 4.1 Carbon dioxide $\left(\mathrm{CO}_{2}\right)$ emissions by sector and total non- $\mathrm{CO}_{2}$ greenhouse gas (GHG) emissions (Kyoto gases) across sectors in baseline (left panel) and mitigation scenarios that reach about 450 (430 to 480 ) ppm $\mathrm{CO}_{2}$-eq (likely to limit warming to $2^{\circ} \mathrm{C}$ above pre-industrial levels) with carbon dioxide capture and storage (CCS, middle panel) and without CCS (right panel). Light yellow background denotes direct $\mathrm{CO}_{2}$ and non- $\mathrm{CO}_{2} \mathrm{GHG}$ emissions for both the baseline and mitigation scenarios. In addition, for the baseline scenarios, the sum of direct and indirect emissions from the energy end-use sectors (transport, buildings and industry) is also shown (dark yellow background). Mitigation scenarios show direct emissions only. However, mitigation in the end-use sectors leads also to indirect emissions reductions in the upstream energy supply sector. Direct emissions of the enduse sectors thus do not include the emission reduction potential at the supply-side due to, for example, reduced electricity demand. Note that for calculating the indirect emissions only electricity emissions are allocated from energy supply to end-use sectors. The numbers at the bottom of the graphs refer to the number of scenarios included in the range, which differs across sectors and time due to different sectoral resolution and time horizon of models. Note that many models cannot reach concentrations of about 450 ppm $\mathrm{CO}_{2}$-eq by 2100 in the absence of CCS, resulting in a low number of scenarios for the right panel. Negative emissions in the electricity sector are due to the application of bioenergy with carbon dioxide capture and storage (BECCS). 'Net' agriculture, forestry and other land use (AFOLU) emissions consider afforestation, reforestation as well as deforestation activities. \{WGIII Figure SPM.7, Figure TS.15\}

industry. An overview of sectoral mitigation options and potentials is provided in Table 4.4. \{WGIII TS 3.2.1\}

Well-designed systemic and cross-sectoral mitigation strategies are more cost-effective in cutting emissions than a focus on individual technologies and sectors with efforts in one sector affecting the need for mitigation in others (medium confidence). In baseline scenarios without new mitigation policies, GHG emissions are projected to grow in all sectors, except for net $\mathrm{CO}_{2}$ emissions in the AFOLU sector (Figure 4.1, left panel). Mitigation scenarios reaching around $450 \mathrm{ppm} \mathrm{CO}$-eq ${ }^{42}$ concentration by $2100^{43}$ (likely to limit warming to $2^{\circ} \mathrm{C}$ above pre-industrial levels) show largescale global changes in the energy supply sector (Figure 4.1, middle and right panel). While rapid decarbonization of energy supply generally entails more flexibility for end-use and AFOLU sectors, stronger demand reductions lessen the mitigation challenge for the supply side of the energy system (Figures 4.1 and 4.2). There are thus strong interdependencies across sectors and the resulting distribution of the mitigation effort is strongly influenced by the availability and performance of future technologies, particularly BECCS and large scale afforestation (Figure 4.1, middle and right panel). The next two decades present a window of opportunity for mitigation in urban areas, as a large portion of the world's urban areas will be developed during this period. \{WGIII SPM.4.2, TS.3.2\}

Decarbonizing (i.e., reducing the carbon intensity of) electricity generation is a key component of cost-effective mitigation strategies in achieving low stabilization levels (of about 450 to about $500 \mathrm{ppm} \mathrm{CO}$-eq, at least about as likely as not to limit warming to $2^{\circ} \mathrm{C}$ above pre-industrial levels) (medium evidence, high agreement). In most integrated modelling scenarios, decarbonization happens more rapidly in electricity generation than in the industry, buildings and transport sectors. In scenarios reaching $450 \mathrm{ppm} \mathrm{CO}_{2}$-eq concentrations by 2100 , global $\mathrm{CO}_{2}$ emissions from the energy supply sector are projected to decline over the next decade and are characterized by reductions of $90 \%$ or more below 2010 levels between 2040 and 2070. \{WGIII SPM.4.2, 6.8, 7.11\}

Efficiency enhancements and behavioural changes, in order to reduce energy demand compared to baseline scenarios without compromising development, are a key mitigation strategy in scenarios reaching atmospheric $\mathrm{CO}_{2}$-eq concentrations of about 450 to about 500 ppm by 2100 (robust evidence, high agreement). Near-term reductions in energy demand are an important

\footnotetext{
42 See Glossary for definition of $\mathrm{CO}_{2}$-eq concentrations and emissions; also Box 3.2 for metrics to calculate the $\mathrm{CO}_{2}$-equivalence of non- $\mathrm{CO}_{2}$ emissions and their influence on sectoral abatement strategies.

43 For comparison, the $\mathrm{CO}_{2}$-eq concentration in 2011 is estimated to be 430 [340 to 520] ppm.
} 
Liquids and hydrogen

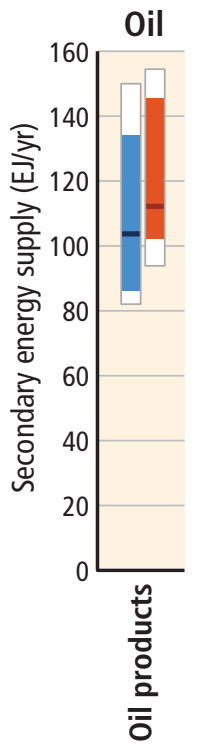

Other liquids and $\mathrm{H}_{2}$

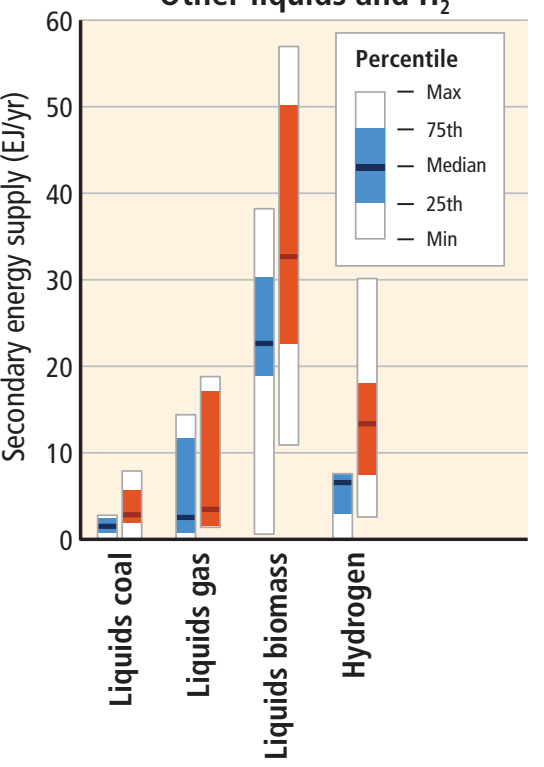

Electricity generation

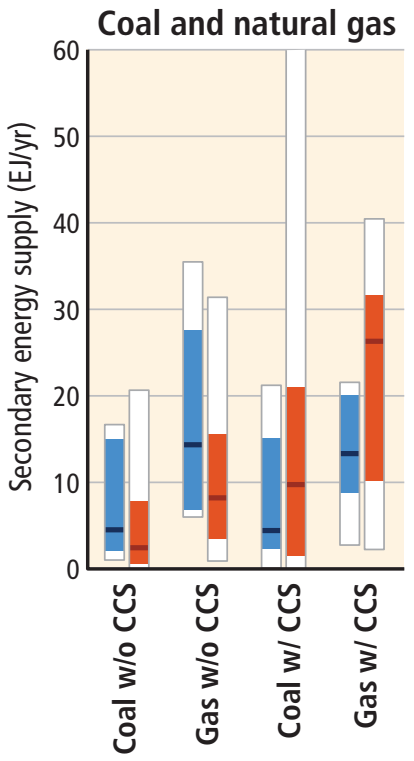

\begin{tabular}{l|l|l|l}
\hline 1 & 2 & 3 & 4 \\
\hline $\begin{array}{l}\text { High energy } \\
\text { demand scenarios } \\
\text { show higher levels } \\
\text { of oil supply. }\end{array}$ & $\begin{array}{l}\text { In high energy demand scenarios, alternative } \\
\text { liquid and hydrogen technologies are scaled } \\
\text { up more rapidly. }\end{array}$ & $\begin{array}{l}\text { High energy demand scenarios show } \\
\text { a more rapid up-scaling of CCS } \\
\text { technologies but a more rapid phase- } \\
\text { out of unabated fossil fuel conversion } \\
\text { technologies. }\end{array}$ & $\begin{array}{l}\text { In high energy demand scenarios non-fossil } \\
\text { electricity generation technologies are scaled up } \\
\text { more rapidly. }\end{array}$ \\
\hline
\end{tabular}

Figure 4.2 Influence of energy demand on the deployment of energy supply technologies in 2050 in mitigation scenarios reaching about 450 to about 500 ppm $\mathrm{CO}_{2}$-eq concentrations by 2100 (at least about as likely as not to limit warming to $2^{\circ} \mathrm{C}$ above pre-industrial levels). Blue bars for 'low energy demand' show the deployment range of scenarios with limited growth in final energy demand of <20\% in 2050 compared to 2010. Red bars show the deployment range of technologies in a case of 'high energy demand' (>20\% growth in 2050 compared to 2010). For each technology, the median, interquartile and full deployment range is displayed. Notes: Scenarios assuming technology restrictions are excluded. Ranges include results from many different integrated models. Multiple scenario results from the same model were averaged to avoid sampling biases. \{WGIII Figure TS. 16\}

element of cost-effective mitigation strategies, provide more flexibility for reducing carbon intensity in the energy supply sector, hedge against related supply-side risks, avoid lock-in to carbon-intensive infrastructures and are associated with important co-benefits (Figure 4.2, Table 4.4). Emissions can be substantially lowered through changes in consumption patterns (e.g., mobility demand and mode, energy use in households, choice of longer-lasting products) and dietary change and reduction in food wastes. A number of options including monetary and non-monetary incentives as well as information measures may facilitate behavioural changes. \{WGIII SPM.4.2\}

Decarbonization of the energy supply sector (i.e., reducing the carbon intensity) requires upscaling of low- and zero-carbon electricity generation technologies (high confidence). In the majority of low-concentration stabilization scenarios (about 450 to about $500 \mathrm{ppm} \mathrm{CO}$-eq , at least about as likely as not to limit warming to $2^{\circ} \mathrm{C}$ above pre-industrial levels), the share of low-carbon electricity supply (comprising renewable energy (RE), nuclear and CCS, including BECCS) increases from the current share of approximately $30 \%$ to more than $80 \%$ by 2050 and $90 \%$ by 2100 , and fossil fuel power generation without CCS is phased out almost entirely by 2100 . Among these low-carbon technologies, a growing number of RE technologies have achieved a level of maturity to enable deployment at significant scale since AR4 (robust evidence, high agreement) and nuclear energy is a mature low-GHG emission source of baseload power, but its share of global electricity generation has been declining (since 1993). GHG emissions from energy supply can be reduced significantly by replacing current world average coal-fired power plants with modern, highly efficient natural gas combined-cycle power plants or combined heat and power plants, provided that natural gas is available and the fugitive emissions associated with extraction and supply are low or mitigated. \{WGIII SPM.4.2\}

Behaviour, lifestyle and culture have a considerable influence on energy use and associated emissions, with high mitigation potential in some sectors, in particular when complementing technological and structural change (medium evidence, medium agreement). In the transport sector, technical and behavioural mitigation measures for all modes, plus new infrastructure and urban redevelopment investments, could reduce final energy demand significantly below baseline levels (robust evidence, medium agreement) (Table 4.4). While opportunities for switching to low-carbon fuels exist, the rate of decarbonization in the transport sector might be constrained by challenges associated with energy storage and the relatively low 
Table 4.4 | Sectoral carbon dioxide $\left(\mathrm{CO}_{2}\right)$ emissions, associated energy system changes and examples of mitigation measures (including for non- $\mathrm{CO}_{2}$ gases; see Box 3.2 for metrics regarding the weighting and abatement of non- $\mathrm{CO}_{2}$ emissions). \{WGIII SPM.7, Figure SPM.8, Table TS.2, 7.11.3, 7.13, 7.14\}

\section{Sectoral $\mathrm{CO}_{2}$ emissions and related energy system changes}
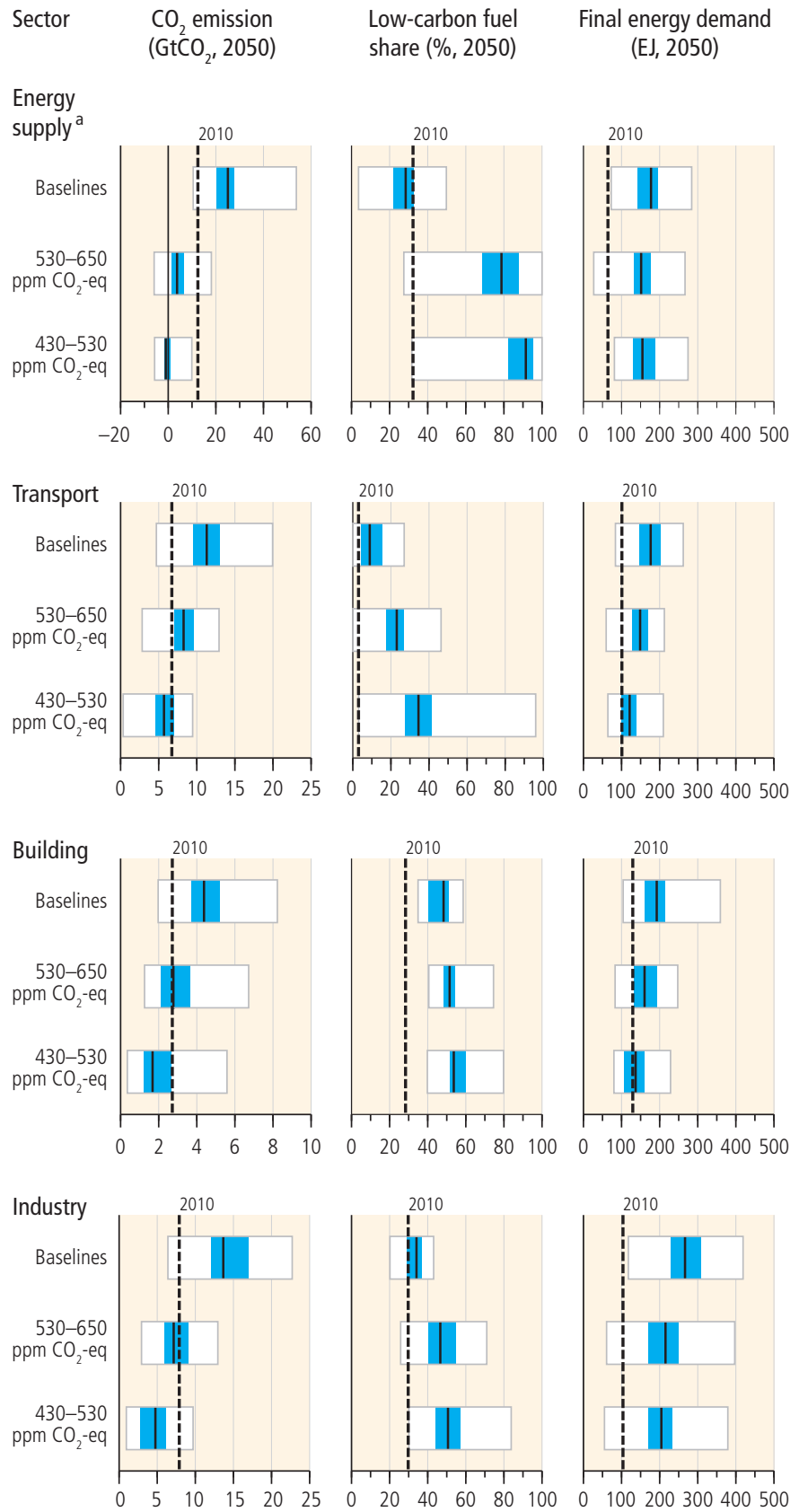

\section{Examples for sectoral mitigation measures}

Key low-carbon $\quad$ Key energy saving options $\quad$ Other options
energy options

$\begin{array}{ll}\begin{array}{l}\text { Renewables (wind, solar } \\ \text { bioenergy, geothermal, hydro, }\end{array} & \text { Energy efficiency improve- } \\ \text { etc.), nuclear, CCS, BECCS, } & \text { technologies, improved } \\ \text { fossil fuel switching } & \text { transmission and distribution, } \\ & \text { CHP and cogeneration }\end{array}$

Fugitive $\mathrm{CH}_{4}$ emissions control

CHP and cogeneration

Fuel switching to low-carbon Efficiency improvements fuels (e.g., hydrogen/electricity (engines, vehicle design, from low-carbon sources), appliances, lighter materials), biofuels modal shift (e.g., from LDVs to public transport or from aviation to HDVs to rail), eco-driving, improved freight logistics, journey avoidance, higher occupancy rates

Building integrated RES, fuel switching to low-carbon fuels (e.g., electricity from low-carbon sources, biofuels)

Device efficiency (heating/cooling systems, water heating, cooking, lighting, appliances), systemic efficiency (integrated design, low/zero energy buildings, district heating/cooling, $\mathrm{CHP}$, smart meters/grids),

behavioural and lifestyle changes (e.g., appliance use, thermostat setting, dwelling size)

\section{Process emissions reductions,} use of waste and CCS in industry, fuel switching among fossil fuels and switch to low-carbon energy (e.g., electricity) or biomass
Energy efficiency and BAT (e.g., furnace/boilers, steam systems, electric motors and control systems, (waste) heat exchanges, recycling), reduction of demand for goods, more intensive use of goods (e.g., improve durability or car sharing)

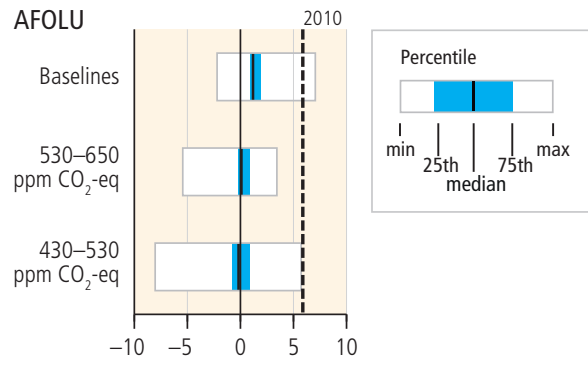

Emissions reduction measures: Methane (e.g., livestock management), nitrous oxide (e.g., fertilizer use), conservation of existing carbon pools (sustainable forest management, reduced deforestation and forest degradation, fire prevention, agroforestry), reduction in emissions intensity
Sequestration options: Substitution options: Demand-side measures: Increasing existing carbon Use of biological products Reduction of loss and pools (e.g., afforestation, instead of fossil/GHG waste of food, changes reforestation, integrated intensive products (e.g., in human diets, use of systems, carbon bioenergy, insulation sequestration in soils) products) long-lived wood products
HFC replacement and leak repair, material efficiency (e.g., process innovation, re-using old materials, product design, etc.) components and appliances, ow energy/GHG intensive construction and materials

a $\mathrm{CO}_{2}$ emissions, low carbon fuel shares, and final energy demand are shown for electricity generation only 
energy density of low-carbon transport fuels (medium confidence). In the building sector, recent advances in technologies, know-how and policies provide opportunities to stabilize or reduce global energy use to about current levels by mid-century. In addition, recent improvements in performance and costs make very low energy construction and retrofits of buildings economically attractive, sometimes even at net negative costs (robust evidence, high agreement). In the industry sector, improvements in GHG emission efficiency and in the efficiency of material use, recycling and reuse of materials and products, and overall reductions in product demand (e.g., through a more intensive use of products) and service demand could, in addition to energy efficiency, help reduce GHG emissions below the baseline level. Prevalent approaches for promoting energy efficiency in industry include information programmes followed by economic instruments, regulatory approaches and voluntary actions. Important options for mitigation in waste management are waste reduction, followed by re-use, recycling and energy recovery (robust evidence, high agreement). \{WGIII SPM.4.2, Box TS.12, TS.3.2\}

The most cost-effective mitigation options in forestry are afforestation, sustainable forest management and reducing deforestation, with large differences in their relative importance across regions. In agriculture, the most cost-effective mitigation options are cropland management, grazing land management and restoration of organic soils (medium evidence, high agreement). About a third of mitigation potential in forestry can be achieved at a cost $<20$ USD/tCO -eq emission. Demand-side measures, such as changes in diet and reductions of losses in the food supply chain, have a significant, but uncertain, potential to reduce GHG emissions from food production (medium evidence, medium agreement). \{WGIII SPM 4.2.4\}

Bioenergy can play a critical role for mitigation, but there are issues to consider, such as the sustainability of practices and the efficiency of bioenergy systems (robust evidence, medium agreement). Evidence suggests that bioenergy options with low lifecycle emissions, some already available, can reduce GHG emissions; outcomes are site-specific and rely on efficient integrated 'biomassto-bioenergy systems', and sustainable land use management and governance. Barriers to large-scale deployment of bioenergy include concerns about GHG emissions from land, food security, water resources, biodiversity conservation and livelihoods. \{WGIII SPM.4.2\}

Mitigation measures intersect with other societal goals, creating the possibility of co-benefits or adverse side-effects. These intersections, if well-managed, can strengthen the basis for undertaking climate mitigation actions (robust evidence, medium agreement). Mitigation can positively or negatively influence the achievement of other societal goals, such as those related to human health, food security, biodiversity, local environmental quality, energy access, livelihoods and equitable sustainable development (see also Section 4.5). On the other hand, policies towards other societal goals can influence the achievement of mitigation and adaptation objectives. These influences can be substantial, although sometimes difficult to quantify, especially in welfare terms. This multi-objective perspective is important in part because it helps to identify areas where support for policies that advance multiple goals will be robust. Potential co-benefits and adverse side effects of the main sectoral mitigation measures are summarized in Table 4.5. Overall, the potential for co-benefits for energy end-use measures outweigh the potential for adverse side effects, whereas the evidence suggests this may not be the case for all energy supply and AFOLU measures. \{WGIII SPM.2\}

\subsection{Policy approaches for adaptation and mitigation, technology and finance}

Effective adaptation and mitigation responses will depend on policies and measures across multiple scales: international, regional, national and sub-national. Policies across all scales supporting technology development, diffusion and transfer, as well as finance for responses to climate change, can complement and enhance the effectiveness of policies that directly promote adaptation and mitigation.

\subsubsection{International and regional cooperation on adaptation and mitigation}

Because climate change has the characteristics of a collective action problem at the global scale (see 3.1), effective mitigation will not be achieved if individual agents advance their own interests independently, even though mitigation can also have local co-benefits. Cooperative responses, including international cooperation, are therefore required to effectively mitigate GHG emissions and address other climate change issues. While adaptation focuses primarily on local to national scale outcomes, its effectiveness can be enhanced through coordination across governance scales, including international cooperation. In fact, international cooperation has helped to facilitate the creation of adaptation strategies, plans, and actions at national, sub-national, and local levels. A variety of climate policy instruments have been employed, and even more could be employed, at international and regional levels to address mitigation and to support and promote adaptation at national and sub-national scales. Evidence suggests that outcomes seen as equitable can lead to more effective cooperation. \{WGII SPM C-1, 2.2, 15.2, WGIII 13.ES, 14.3, 15.8, SREX SPM, 7.ES\}

The United Nations Framework Convention on Climate Change (UNFCCC) is the main multilateral forum focused on addressing climate change, with nearly universal participation. UNFCCC activities since 2007, which include the 2010 Cancún Agreements and the 2011 Durban Platform for Enhanced Action, have sought to enhance actions under the Convention, and have led to an increasing number of institutions and other arrangements for international climate change cooperation. Other institutions organized at different levels of governance have resulted in diversifying international climate change cooperation. \{WGIII SPM.5.2, 13.5\}

Existing and proposed international climate change cooperation arrangements vary in their focus and degree of centralization and coordination. They span: multilateral agreements, harmonized national policies and decentralized but coordinated national policies, as well as regional and regionally-coordinated policies (see Figure 4.3). \{WGIII SPM.5.2\} 


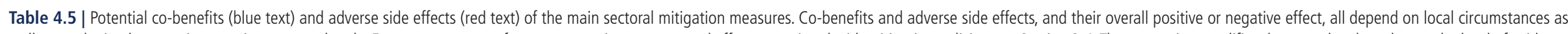

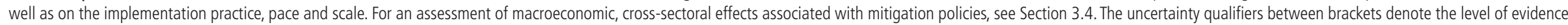
and agreement on the respective effect. Abbreviations for evidence: $\mathrm{I}=$ limited, $\mathrm{m}=$ medium, $\mathrm{r}=$ robust; for agreement: $\mathrm{I}=$ low, $\mathrm{m}=$ medium, $\mathrm{h}=$ high. $\{$ WGIII Table TS.3, Table TS.4, Table TS.5, Table TS.6, Table TS.7, Table 6.7\}

\begin{tabular}{|c|c|c|c|}
\hline \multirow{2}{*}{ Sectoral mitigation measures } & \multicolumn{3}{|l|}{ Effect on additional objectives/concerns } \\
\hline & Economic & Social & Environmental \\
\hline Energy Supply & \multicolumn{3}{|c|}{ For possible upstream effects of biomass supply for bioenergy, see AFOLU. } \\
\hline Nuclear replacing coal power & \begin{tabular}{|l|} 
Energy security (reduced exposure to fuel price volatility) \\
$(\boldsymbol{m} / \boldsymbol{m})$; local employment impact (but uncertain net effect) \\
$(\mathrm{I} \boldsymbol{m})$; legacy/cost of waste and abandoned reactors $(\boldsymbol{m} / \boldsymbol{h})$ \\
\end{tabular} & $\begin{array}{l}\text { Mixed health impact via reduced air pollution and coal mining } \\
\text { accidents }(\boldsymbol{m} / \boldsymbol{h}) \text {, nuclear accidents and waste treatment, uranium } \\
\text { mining and milling }(\boldsymbol{m} / \mathbf{l}) \text {; safety and waste concerns }(\boldsymbol{r} / \boldsymbol{h}) \text {; prolifera- } \\
\text { tion risk }(\boldsymbol{m} / \mathbf{m})\end{array}$ & $\begin{array}{l}\text { Mixed ecosystem impact via reduced air pollution }(\boldsymbol{m} / \boldsymbol{h}) \text { and coal } \\
\text { mining }(/ / \boldsymbol{h}) \text {, nuclear accidents }(\boldsymbol{m} / \boldsymbol{m})\end{array}$ \\
\hline $\begin{array}{l}\text { Renewable energy (wind, PV, CSP, } \\
\text { hydro, geothermal, bioenergy) repla- } \\
\text { cing coal }\end{array}$ & $\begin{array}{l}\text { Energy security }(\boldsymbol{r} / \boldsymbol{m}) \text {; local employment (but uncertain net } \\
\text { effect) }(\boldsymbol{m} / \boldsymbol{m}) \text {; water management (for some hydro energy) } \\
(\boldsymbol{m} / \boldsymbol{h}) \text {; extra measures to match demand (for PV, wind, some } \\
\text { CSP) }(r / h) \text {; higher use of critical metals for PV and direct drive } \\
\text { wind turbines }(\boldsymbol{r} / \boldsymbol{m})\end{array}$ & $\begin{array}{l}\text { Reduced health impact via reduced air pollution (except bioenergy) } \\
(\boldsymbol{r} / \boldsymbol{h}) \text { and coal mining accidents }(\boldsymbol{m} / \boldsymbol{h}) \text {; contribution to (off-grid) } \\
\text { energy access }(\boldsymbol{m} / \mathbf{l}) \text {; threat of displacement (for large hydro } \\
\text { installations) }(\boldsymbol{m} / \boldsymbol{h})\end{array}$ & $\begin{array}{l}\text { Mixed ecosystem impact via reduced air pollution (except bioe- } \\
\text { nergy) }(\boldsymbol{m} / \boldsymbol{h}) \text { and coal mining }(/ / \boldsymbol{h}) \text {, habitat impact (for some hydro } \\
\text { energy) }(\boldsymbol{m} / \boldsymbol{m}) \text {, landscape and wildlife impact }(\boldsymbol{m} / \boldsymbol{m}) \text {; lower/higher } \\
\text { water use (for wind, PV }(\boldsymbol{m} / \boldsymbol{m}) \text {; bioenergy, CSP, geothermal and } \\
\text { reservoir hydro }(\boldsymbol{m} / \boldsymbol{h}))\end{array}$ \\
\hline Fossil energy with CCS replacing coal & $\begin{array}{l}\text { Preservation vs. lock-in of human and physical capital in the } \\
\text { fossil industry }(\boldsymbol{m} / \boldsymbol{m}) \text {; long-term monitoring of } \mathrm{CO}_{2} \text { storage } \\
(\boldsymbol{m} / \boldsymbol{h})\end{array}$ & $\begin{array}{l}\text { Health impact via risk of } \mathrm{CO}_{2} \text { leakage }(\boldsymbol{m} / \boldsymbol{m}) \text { and additional } \\
\text { upstream supply-chain activities }(\boldsymbol{m} / \boldsymbol{h}) \text {; safety concerns }\left(\mathrm{CO}_{2}\right. \\
\text { storage and transport) }(\boldsymbol{m} / \boldsymbol{h})\end{array}$ & $\begin{array}{l}\text { Ecosystem impact via additional upstream supply-chain activities } \\
(\boldsymbol{m} / \boldsymbol{m}) \text { and higher water use }(\boldsymbol{m} / \boldsymbol{h})\end{array}$ \\
\hline $\begin{array}{l}\mathrm{CH}_{4} \text { leakage prevention, capture or } \\
\text { treatment }\end{array}$ & Energy security (potential to use gas in some cases) (I/h) & $\begin{array}{l}\text { Reduced health impact via reduced air pollution }(\boldsymbol{m} / \boldsymbol{m}) \text {; occupatio- } \\
\text { nal safety at coal mines }(\boldsymbol{m} / \boldsymbol{m})\end{array}$ & Reduced ecosystem impact via reduced air pollution $(/ / m)$ \\
\hline Transport & \multicolumn{3}{|c|}{ For possible upstream effects of low-carbon electricity, see Energy Supply. For biomass supply, see AFOLU. } \\
\hline Reduction of carbon intensity of fuel & \begin{tabular}{|l||}
$\begin{array}{l}\text { Energy security (diversification, reduced oil dependence } \\
\text { and exposure to oil price volatility) }(\boldsymbol{m} / \boldsymbol{m}) \text {; technological } \\
\text { spillovers (I/) }\end{array}$ \\
\end{tabular} & $\begin{array}{l}\text { Mixed health impact via increased/reduced urban air pollution by } \\
\text { electricity and hydrogen }(\boldsymbol{r} / \boldsymbol{h}) \text {, diesel }(I / \boldsymbol{m}) \text {; road safety concerns } \\
(I / /) \text { but reduced health impact via reduced noise }(/ / m) \text { of electric } \\
\text { LDVs }\end{array}$ & $\begin{array}{l}\text { Mixed ecosystem impact of electricity and hydrogen via reduced } \\
\text { urban air pollution }(\boldsymbol{m} / \mathbf{m}) \text { and material use (unsustainable mining) } \\
(\mathrm{I} / \mathrm{I})\end{array}$ \\
\hline Reduction of energy intensity & $\begin{array}{l}\text { Energy security (reduced oil dependence and exposure to oil } \\
\text { price volatility) }(\boldsymbol{m} / \boldsymbol{m})\end{array}$ & $\begin{array}{l}\text { Reduced health impact via reduced urban air pollution }(\boldsymbol{r} / \boldsymbol{h}) ; \\
\text { road safety (crash-worthiness depending on the design of the } \\
\text { standards) }(\boldsymbol{m} / \boldsymbol{m})\end{array}$ & $\begin{array}{l}\text { Reduced ecosystem and biodiversity impact via reduced urban air } \\
\text { pollution }(\boldsymbol{m} / \mathbf{h})\end{array}$ \\
\hline $\begin{array}{l}\text { Compact urban form and improved } \\
\text { transport infrastructure } \\
\text { Modal shift }\end{array}$ & $\begin{array}{l}\text { Energy security (reduced oil dependence and exposure to oil } \\
\text { price volatility) }(\boldsymbol{m} / \boldsymbol{m}) \text {; productivity (reduced urban conge- } \\
\text { stion and travel times, affordable and accessible transport) } \\
(\boldsymbol{m} / \boldsymbol{h})\end{array}$ & $\begin{array}{l}\text { Mixed health impact for non-motorized modes via increased physi- } \\
\text { cal activity }(\boldsymbol{r} / \boldsymbol{h}) \text {, potentially higher exposure to air pollution }(\boldsymbol{r} / \boldsymbol{h}) \text {, } \\
\text { reduced noise (via modal shift and travel reduction) }(\boldsymbol{r} / \boldsymbol{h}) \text {; equitable } \\
\text { mobility access to employment opportunities }(\boldsymbol{r} / \boldsymbol{h}) \text {; road safety (via } \\
\text { modal shift) }(\boldsymbol{r} / \boldsymbol{h})\end{array}$ & $\begin{array}{l}\text { Reduced ecosystem impact via reduced urban air pollution }(\boldsymbol{r} / \mathbf{h}) \\
\text { and land use competition }(\boldsymbol{m} / \boldsymbol{m})\end{array}$ \\
\hline $\begin{array}{l}\text { Journey distance reduction and } \\
\text { avoidance }\end{array}$ & \begin{tabular}{|l|} 
Energy security (reduced oil dependence and exposure to oil \\
price volatility) $(\boldsymbol{r} / \boldsymbol{h}) ;$ productivity (reduced urban congestion/ \\
travel times, walking) $(\boldsymbol{r} / \boldsymbol{h})$
\end{tabular} & Reduced health impact (for non-motorized transport modes) $(\boldsymbol{r} / \boldsymbol{h})$ & $\begin{array}{l}\text { Mixed ecosystem impact via reduced urban air pollution }(\boldsymbol{r} / \boldsymbol{h}) \text {, new/ } \\
\text { shorter shipping routes }(\boldsymbol{r} / \boldsymbol{h}) \text {; reduced land use competition from } \\
\text { transport infrastructure }(\boldsymbol{r} / \boldsymbol{h})\end{array}$ \\
\hline Buildings & \multicolumn{3}{|c|}{ For possible upstream effects of fuel switching and RES, see Energy Supply. } \\
\hline $\begin{array}{l}\text { Reduction of GHG emissions intensity } \\
\text { (e.g., fuel switching, RES incorporation, } \\
\text { green roofs) }\end{array}$ & \begin{tabular}{|l|} 
Energy security $(\boldsymbol{m} / \boldsymbol{h})$; employment impact $(\boldsymbol{m} / \boldsymbol{m})$; lower \\
need for energy subsidies $(/ / /)$; asset values of buildings $(/ / \boldsymbol{m})$
\end{tabular} & $\begin{array}{l}\text { Fuel poverty alleviation via reduced energy demand }(\boldsymbol{m} / \boldsymbol{h}) \text {; energy } \\
\text { access (for higher energy cost) }(I / \boldsymbol{m}) \text {; productive time for women/ } \\
\text { children (for replaced traditional cookstoves) }(\boldsymbol{m} / \boldsymbol{h})\end{array}$ & $\begin{array}{l}\text { Reduced health impact in residential buildings and ecosystem } \\
\text { impact (via reduced fuel poverty }(\boldsymbol{r} / \boldsymbol{h}) \text {, indoor/outdoor air pollution } \\
(\boldsymbol{r} / \mathbf{h}) \text { and UHI effect) }(\mathbf{I} \boldsymbol{m}) \text {; urban biodiversity (for green roofs) } \\
(\boldsymbol{m} / \mathbf{m})\end{array}$ \\
\hline $\begin{array}{l}\text { Retrofits of existing buildings } \\
\text { Exemplary new buildings } \\
\text { Efficient equipment }\end{array}$ & $\begin{array}{l}\text { Energy security }(\boldsymbol{m} / \boldsymbol{h}) \text {; employment impact }(\boldsymbol{m} / \boldsymbol{m}) \text {; pro- } \\
\text { ductivity (for commercial buildings) }(\boldsymbol{m} / \boldsymbol{h}) \text {; less need for } \\
\text { energy subsidies }(\mathbf{l} / \mathbf{l}) \text {; asset value of buildings }(\boldsymbol{I} \boldsymbol{m}) \text {; disaster } \\
\text { resilience }(\boldsymbol{I} \boldsymbol{m})\end{array}$ & $\begin{array}{l}\text { Fuel poverty alleviation via reduced energy demand (for retrofits } \\
\text { and efficient equipment) }(\boldsymbol{m} / \boldsymbol{h}) \text {; energy access (higher housing } \\
\text { cost) }(\boldsymbol{l} / \boldsymbol{m}) \text {; thermal comfort }(\boldsymbol{m} / \boldsymbol{h}) \text {; productive time for women and } \\
\text { children (for replaced traditional cookstoves) }(\boldsymbol{m} / \boldsymbol{h})\end{array}$ & $\begin{array}{l}\text { Reduced health and ecosystem impact (e.g., via reduced fuel } \\
\text { poverty }(\boldsymbol{r} / \boldsymbol{h}) \text {, indoor/outdoor air pollution }(\boldsymbol{r} / \boldsymbol{h}), \text { UHI effect }(\mathbf{I} \boldsymbol{m}), \\
\text { improved indoor environmental conditions }(\boldsymbol{m} / \boldsymbol{h}) \text { ); health risk via } \\
\text { insufficient ventilation }(\boldsymbol{m} / \boldsymbol{m}) \text {; reduced water consumption and } \\
\text { sewage production }(\boldsymbol{I} / \mathbf{l})\end{array}$ \\
\hline
\end{tabular}




\begin{tabular}{|c|c|c|c|}
\hline \multirow{2}{*}{ Sectoral mitigation measures } & \multicolumn{3}{|l|}{ Effect on additional objectives/concerns } \\
\hline & Economic & Social & Environmental \\
\hline $\begin{array}{l}\text { Behavioural changes reducing energy } \\
\text { demand }\end{array}$ & Energy security $(\boldsymbol{m} / \boldsymbol{h})$; less need for energy subsidies $(\mathbf{I} / \mathbf{l})$ & & $\begin{array}{l}\text { Reduced health and ecosystem impact (e.g., via improved indoor } \\
\text { environmental conditions }(\boldsymbol{m} / \boldsymbol{h}) \text { and less outdoor air pollution }(\boldsymbol{r} / \boldsymbol{h}))\end{array}$ \\
\hline Industry & \multicolumn{3}{|c|}{ For possible upstream effects of low-carbon energy supply (incl. CCS), see Energy Supply and of biomass supply, see AFOLU. } \\
\hline $\begin{array}{l}\text { Reduction of } \mathrm{CO}_{2} / \text { non- } \mathrm{CO}_{2} \mathrm{GHG} \\
\text { emission intensity }\end{array}$ & Competitiveness and productivity $(\boldsymbol{m} / \boldsymbol{h})$ & \begin{tabular}{|l|} 
Reduced health impact via reduced local air pollution and better \\
working conditions (PFC from aluminium) $(\boldsymbol{m} / \boldsymbol{m})$
\end{tabular} & $\begin{array}{l}\text { Reduced ecosystem impact (via reduced local air and water polluti- } \\
\text { on) }(\boldsymbol{m} / \boldsymbol{m}) \text {; water conservation }(\boldsymbol{l} / \boldsymbol{m})\end{array}$ \\
\hline $\begin{array}{l}\text { Technical energy efficiency improve- } \\
\text { ments via new processes/technologies }\end{array}$ & $\begin{array}{l}\text { Energy security (via lower energy intensity) }(\boldsymbol{m} / \boldsymbol{m}) \text {; employ- } \\
\text { ment impact }(\boldsymbol{I} / \mathbf{l}) \text {; competitiveness and productivity }(\boldsymbol{m} / \boldsymbol{h}) ; \\
\text { technological spillovers in DCs }(\boldsymbol{I} / \mathbf{I})\end{array}$ & $\begin{array}{l}\text { Reduced health impact via reduced local pollution }(\mathbf{I} \boldsymbol{m}) \text {; new busi- } \\
\text { ness opportunities }(\boldsymbol{m} / \boldsymbol{m}) \text {; increased water availability and quality } \\
(\mathrm{I} / \mathbf{I} \text {; improved safety, working conditions and job satisfaction } \\
(\boldsymbol{m} / \mathbf{m})\end{array}$ & $\begin{array}{l}\text { Reduced ecosystem impact via reduced fossil fuel extraction (I/I) } \\
\text { and reduced local pollution and waste }(\boldsymbol{m} / \boldsymbol{m})\end{array}$ \\
\hline Material efficiency of goods, recycling & $\begin{array}{l}\text { Decreased national sales tax revenue in the medium term } \\
(I / I) \text {; employment impact (waste recycling) }(I / I) \text {; competitive- } \\
\text { ness in manufacturing }(I / I) \text {; new infrastructure for industrial } \\
\text { clusters }(I / /)\end{array}$ & $\begin{array}{l}\text { Reduced health impacts and safety concerns }(/ / m) \text {; new business } \\
\text { opportunities }(\boldsymbol{m} / \boldsymbol{m}) \text { and reduced local conflicts (reduced resource } \\
\text { extraction) }(I / m)\end{array}$ & $\begin{array}{l}\text { Reduced ecosystem impact via reduced local air and water pollu- } \\
\text { tion and waste material disposal }(\mathbf{m} / \mathbf{m}) \text {; reduced use of raw/virgin } \\
\text { materials and natural resources implying reduced unsustainable } \\
\text { resource mining }(\mathbf{l} / \mathbf{l})\end{array}$ \\
\hline Product demand reductions & $\begin{array}{l}\text { Decreased national sales tax revenue in the medium term } \\
(I / /)\end{array}$ & Increased wellbeing via diverse lifestyle choices (I//) & Reduced post-consumption waste $(I / I)$ \\
\hline AFOLU & \multicolumn{3}{|c|}{ Note: co-benefits and adverse side effects depend on the development context and the scale of the intervention (size). } \\
\hline \multirow[t]{2}{*}{$\begin{array}{l}\text { Supply side: forestry, land-based agri- } \\
\text { culture, livestock, integrated systems } \\
\text { and bioenergy }\end{array}$} & \multirow{2}{*}{$\begin{array}{l}\text { Mixed employment impact via entrepreneurship develop- } \\
\text { ment }(\boldsymbol{m} / \boldsymbol{h}) \text {, use of less labour-intensive technologies in agri- } \\
\text { culture }(\boldsymbol{m} / \boldsymbol{m}) \text {; diversification of income sources and access } \\
\text { to markets }(\boldsymbol{r} / \boldsymbol{h}) \text {; additional income to sustainable landscape } \\
\text { management }(\boldsymbol{m} / \boldsymbol{h}) \text {; income concentration }(\boldsymbol{m} / \boldsymbol{m}) \text {; energy } \\
\text { security (resource sufficiency) }(\boldsymbol{m} / \boldsymbol{h}) \text {; Innovative financing } \\
\text { mechanisms for sustainable resource management }(\boldsymbol{m} / \boldsymbol{h}) \text {; } \\
\text { technology innovation and transfer }(\boldsymbol{m} / \boldsymbol{m})\end{array}$} & \multirow{2}{*}{$\begin{array}{l}\text { Increased food-crops production through integrated systems } \\
\text { and sustainable agriculture intensification }(\boldsymbol{r} / \boldsymbol{m}) \text {; decreased food } \\
\text { production (locally) due to large-scale monocultures of non-food } \\
\text { crops }(\boldsymbol{r} / \mathbf{l}) \text {; increased cultural habitats and recreational areas } \\
\text { via (sustainable) forest management and conservation }(\boldsymbol{m} / \boldsymbol{m}) \text {; } \\
\text { improved human health and animal welfare (e.g., through less } \\
\text { use of pesticides, reduced burning practices and agroforestry } \\
\text { and silvo-pastoral systems) }(\boldsymbol{m} / \boldsymbol{h}) \text {; human health impact related } \\
\text { to burning practices (in agriculture or bioenergy) }(\boldsymbol{m} / \boldsymbol{m}) \text {; mixed } \\
\text { impacts on gender, intra- and inter-generational equity via parti- } \\
\text { cipation and fair benefit sharing }(\boldsymbol{r} / \boldsymbol{h}) \text { and higher concentration of } \\
\text { benefits }(\boldsymbol{m} / \mathbf{m})\end{array}$} & $\begin{array}{l}\text { Mixed impact on ecosystem services via large-scale monocultures } \\
(\boldsymbol{r} / \boldsymbol{h}) \text {, ecosystem conservation, sustainable management as well } \\
\text { as sustainable agriculture }(\boldsymbol{r} / \boldsymbol{h}) \text {; increased land use competition } \\
(\boldsymbol{r} / \boldsymbol{m}) \text {; increased soil quality }(\boldsymbol{r} / \boldsymbol{h}) \text {; decreased erosion }(\boldsymbol{r} / \boldsymbol{h}) \text {; increased } \\
\text { ecosystem resilience }(\boldsymbol{m} / \boldsymbol{h}) \text {; albedo and evaporation }(\boldsymbol{r} / \boldsymbol{h})\end{array}$ \\
\hline & & & $\begin{array}{l}\text { Institutional aspects: mixed impact on tenure and use rights at } \\
\text { the local level (for indigenous people and local communities) }(r / h) \\
\text { and on access to participative mechanisms for land management } \\
\text { decisions }(\boldsymbol{r} / \boldsymbol{h}) \text {; enforcement of existing policies for sustainable } \\
\text { resource management }(\boldsymbol{r} / \boldsymbol{h})\end{array}$ \\
\hline $\begin{array}{l}\text { Human Settlements and Infra- } \\
\text { structure }\end{array}$ & \multicolumn{3}{|c|}{ For compact urban form and improved transport infrastructure, see also Transport. } \\
\hline $\begin{array}{l}\text { Compact development and infra- } \\
\text { structure }\end{array}$ & $\begin{array}{l}\text { Increased innovation and efficient resource use }(\boldsymbol{r} / \boldsymbol{h}) \text {; higher } \\
\text { rents and property values }(\boldsymbol{m} / \boldsymbol{m})\end{array}$ & Improved health from increased physical activity: see Transport & Preservation of open space $(\boldsymbol{m} / \boldsymbol{m})$ \\
\hline Increased accessibility & Commute savings $(\boldsymbol{r} / \boldsymbol{h})$ & $\begin{array}{l}\text { Improved health from increased physical activity: see Transport; } \\
\text { increased social interaction and mental health }(\boldsymbol{m} / \boldsymbol{m}) \\
\end{array}$ & $\begin{array}{l}\text { Improved air quality and reduced ecosystem and health impacts } \\
(\boldsymbol{m} / \boldsymbol{h})\end{array}$ \\
\hline Mixed land use & $\begin{array}{l}\text { Commute savings }(r / h) ; \text { higher rents and property values } \\
(\boldsymbol{m} / \boldsymbol{m})\end{array}$ & $\begin{array}{l}\text { Improved health from increased physical activity }(\boldsymbol{r} / \boldsymbol{h}) \text {; social } \\
\text { interaction and mental health }(\mathbf{I} \boldsymbol{m})\end{array}$ & $\begin{array}{l}\text { Improved air quality and reduced ecosystem and health impacts } \\
(\boldsymbol{m} / \mathbf{h})\end{array}$ \\
\hline
\end{tabular}




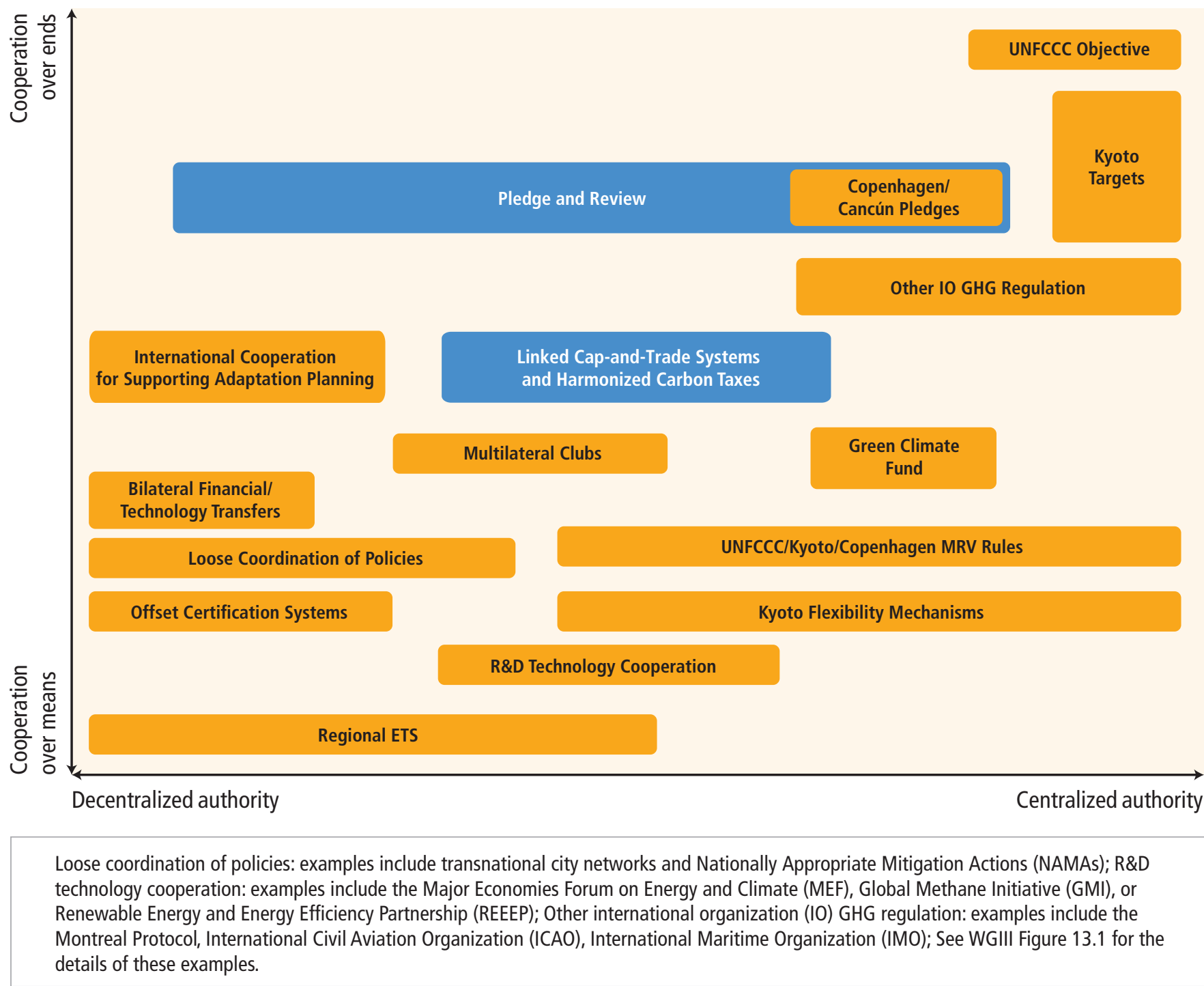

Figure 4.3 | Alternative forms of international cooperation. The figure represents a compilation of existing and possible forms of international cooperation, based upon a survey of published research, but is not intended to be exhaustive of existing or potential policy architectures, nor is it intended to be prescriptive. Examples in orange are existing agreements. Examples in blue are structures for agreements proposed in the literature. The width of individual boxes indicates the range of possible degrees of centralization for a particular agreement. The degree of centralization indicates the authority an agreement confers on an international institution, not the process of negotiating the agreement. \{WGIII Figure 13.2\}

While a number of new institutions are focused on adaptation funding and coordination, adaptation has historically received less attention than mitigation in international climate policy (robust evidence, medium agreement). Inclusion of adaptation is increasingly important to reduce the risk from climate change impacts and may engage a greater number of countries. \{WGIII 13.2, 13.3.3, 13.5.1.1, 13.14\}

The Kyoto Protocol offers lessons towards achieving the ultimate objective of the UNFCCC, particularly with respect to participation, implementation, flexibility mechanisms, and environmental effectiveness (medium evidence, low agreement). The Protocol was the first binding step toward implementing the principles and goals provided by the UNFCCC. According to national GHG inventories through 2012 submitted to the UNFCCC by October 2013, Annex B Parties with quantified emission limitations (and reduction obligations) in aggregate may have bettered their collective emission reduction target in the first commitment period ${ }^{44}$ but some emissions reductions that would have occurred even in its absence were also counted. The Protocol's Clean Development Mechanism (CDM) created a market for emissions offsets from developing countries, the purpose being two-fold: to help Annex I countries fulfill their commitments and to assist non-Annex I countries achieve sustainable development. The CDM generated Certified Emission Reductions (offsets) equivalent to emissions of over $1.4 \mathrm{GtCO}_{2}$-eq ${ }^{42}$ by October 2013 , led to significant project investments, and generated investment flows for a variety of functions, including the UNFCCC Adaptation Fund. However, its environmental effectiveness has been questioned by some, particularly

44 The final conclusion regarding compliance of Annex B Parties remains subject to the review process under the Kyoto Protocol as of October 2014. 
in regard to its early years, due to concerns about the additionality of projects (that is, whether projects bring about emissions that are different from business as usual (BAU) circumstances), the validity of baselines, and the possibility of emissions leakage (medium evidence, medium agreement). Such concerns about additionality are common to any emission-reduction-credit (offset) program, and are not specific to the CDM. Due to market forces, the majority of single CDM projects have been concentrated in a limited number of countries, while Programmes of Activities, though less frequent, have been more evenly distributed. In addition, the Kyoto Protocol created two other 'flexibility mechanisms': Joint Implementation and International Emissions Trading. \{WGIII SPM.5.2, Table TS.9, 13.7, 13.13.1.1, 14.3\}

Several conceptual models for effort-sharing have been identified in research. However, realized distributional impacts from actual international cooperative agreements depend not only on the approach taken but also on criteria applied to operationalize equity and the manner in which developing countries' emissions reduction plans are financed. $\{W G I I I 4.6,13.4\}$

Policy linkages among regional, national and sub-national climate policies offer potential climate change mitigation benefits (medium evidence, medium agreement). Linkages have been established between carbon markets and in principle could also be established between and among a heterogeneous set of policy instruments including non-market-based policies, such as performance standards. Potential advantages include lower mitigation costs, decreased emission leakage and increased market liquidity. \{WGIII SPM.5.2, 13.3, 13.5, 13.6, 13.7, 14.5\}

Regional initiatives between national and global scales are being developed and implemented, but their impact on global mitigation has been limited to date (medium confidence). Some climate policies could be more environmentally and economically effective if implemented across broad regions, such as by embodying mitigation objectives in trade agreements or jointly constructing infrastructures that facilitate reduction in carbon emissions. \{WGIII Table TS.9, 13.13, 14.4, 14.5\}

International cooperation for supporting adaptation planning and implementation has assisted in the creation of adaptation strategies, plans and actions at national, sub-national and local levels (high confidence). For example, a range of multilateral and regionally targeted funding mechanisms have been established for adaptation; UN agencies, international development organizations and non-governmental organisations (NGOs) have provided information, methodologies and guidelines; and global and regional initiatives supported and promoted the creation of national adaptation strategies in both developing and developed countries. Closer integration of disaster risk reduction and climate change adaptation at the international level, and the mainstreaming of both into international development assistance, may foster greater efficiency in the use of resources and capacity. However, stronger efforts at the international level do not necessarily lead to substantive and rapid results at the local level. \{WGII 15.2, 15.3, SREX SPM, 7.4, 8.2, 8.5\}

\subsubsection{National and sub-national policies}

\subsubsection{Adaptation}

Adaptation experience is accumulating across regions in the public and private sector and within communities (high confidence). Adaptation options adopted to date (see Table 4.6) emphasize incremental adjustments and co-benefits and are starting to emphasize flexibility and learning (medium evidence, medium agreement). Most assessments of adaptation have been restricted to impacts, vulnerability and adaptation planning, with very few assessing the processes of implementation or the effects of adaptation actions (medium evidence, high agreement). \{WGII SPM A-2, TS A-2\}

Table 4.6 | Recent adaptation actions in the public and private sector across regions. \{WGII SPM A-2\}

\begin{tabular}{|c|c|}
\hline Region & Example of actions \\
\hline Africa & $\begin{array}{l}\text { Most national governments are initiating governance systems for adaptation. Disaster risk management, adjustments in technologies and infrastructure, } \\
\text { ecosystem-based approaches, basic public health measures and livelihood diversification are reducing vulnerability, although efforts to date tend to be } \\
\text { isolated. }\end{array}$ \\
\hline Asia & $\begin{array}{l}\text { Adaptation is being facilitated in some areas through mainstreaming climate adaptation action into sub-national development planning, early warning } \\
\text { systems, integrated water resources management, agroforestry and coastal reforestation of mangroves. }\end{array}$ \\
\hline North America & $\begin{array}{l}\text { Governments are engaging in incremental adaptation assessment and planning, particularly at the municipal level. Some proactive adaptation is } \\
\text { occurring to protect longer-term investments in energy and public infrastructure. }\end{array}$ \\
\hline $\begin{array}{l}\text { Central and } \\
\text { South America }\end{array}$ & $\begin{array}{l}\text { Ecosystem-based adaptation including protected areas, conservation agreements and community management of natural areas is occurring. Resilient } \\
\text { crop varieties, climate forecasts and integrated water resources management are being adopted within the agricultural sector in some areas. }\end{array}$ \\
\hline The Ocean & $\begin{array}{l}\text { International cooperation and marine spatial planning are starting to facilitate adaptation to climate change, with constraints from challenges of spatial } \\
\text { scale and governance issues. }\end{array}$ \\
\hline
\end{tabular}


National governments play key roles in adaptation planning and implementation (robust evidence, high agreement). There has been substantial progress since the AR4 in the development of national adaptation strategies and plans. This includes National Adaptation Programmes of Action (NAPAs) by least developed countries, the National Adaptation Plan (NAP) process, and strategic frameworks for national adaptation in Organisation for Economic Co-operation and Development (OECD) countries. National governments can coordinate adaptation efforts of local and sub-national governments, for example by protecting vulnerable groups, by supporting economic diversification, and by providing information, policy and legal frameworks and financial support. \{WGII SPM C-1, 15.2\}

While local government and the private sector have different functions, which vary regionally, they are increasingly recognized as critical to progress in adaptation, given their roles in scaling up adaptation of communities, households and civil society and in managing risk information and financing (medium evidence, high agreement). There is a significant increase in the number of planned adaptation responses at the local level in rural and urban communities of developed and developing countries since the AR4. However, local councils and planners are often confronted by the complexity of adaptation without adequate access to guiding information or data on local vulnerabilities and potential impacts. Steps for mainstreaming adaptation into local decision-making have been identified but challenges remain in their implementation. Hence, scholars stress the important role of linkages with national and sub-national levels of government as well as partnerships among public, civic and private sectors in implementing local adaptation responses. \{WGII SPM A-2, SPM C-1, 14.2, 15.2\}

Institutional dimensions of adaptation governance, including the integration of adaptation into planning and decision-making, play a key role in promoting the transition from planning to implementation of adaptation (robust evidence, high agreement). The most commonly emphasized institutional barriers or enablers for adaptation planning and implementation are: 1) multilevel institutional co-ordination between different political and administrative levels in society; 2) key actors, advocates and champions initiating, mainstreaming and sustaining momentum for climate adaptation; 3 ) horizontal interplay between sectors, actors and policies operating at similar administrative levels; 4) political dimensions in planning and implementation; and 5) coordination between formal governmental, administrative agencies and private sectors and stakeholders to increase efficiency, representation and support for climate adaptation measures. \{WGII 15.2, 15.5, 16.3, Box 15-1\}

Existing and emerging economic instruments can foster adaptation by providing incentives for anticipating and reducing impacts (medium confidence). Instruments include public-private finance partnerships, loans, payments for environmental services, improved resource pricing, charges and subsidies, norms and regulations and risk sharing and transfer mechanisms. Risk financing mechanisms in the public and private sector, such as insurance and risk pools, can contribute to increasing resilience, but without attention to major design challenges, they can also provide disincentives, cause market failure and decrease equity. Governments often play key roles as regulators, providers or insurers of last resort. \{WGII SPM C-1\}

\subsubsection{Mitigation}

There has been a considerable increase in national and subnational mitigation plans and strategies since AR4. In 2012, 67\% of global GHG emissions ${ }^{42}$ were subject to national legislation or strategies versus $45 \%$ in 2007 . However, there has not yet been a substantial deviation in global emissions from the past trend. These plans and strategies are in their early stages of development and implementation in many countries, making it difficult to assess their aggregate impact on future global emissions (medium evidence, high agreement). \{WGIII SPM.5.1\}

Since AR4, there has been an increased focus on policies designed to integrate multiple objectives, increase co-benefits and reduce adverse side effects (high confidence). Governments often explicitly reference co-benefits in climate and sectoral plans and strategies. \{WGIII SPM.5.1\}

Sector-specific policies have been more widely used than economy-wide policies (Table 4.7) (medium evidence, high agreement). Although most economic theory suggests that economy-wide policies for mitigation would be more cost-effective than sector-specific policies, administrative and political barriers may make economy-wide policies harder to design and implement than sector-specific policies. The latter may be better suited to address barriers or market failures specific to certain sectors and may be bundled in packages of complementary policies \{WGIII SPM.5.1\}

In principle, mechanisms that set a carbon price, including cap and trade systems and carbon taxes, can achieve mitigation in a cost-effective way, but have been implemented with diverse effects due in part to national circumstances as well as policy design. The short-run environmental effects of cap and trade systems have been limited as a result of loose caps or caps that have not proved to be constraining (limited evidence, medium agreement). In some countries, tax-based policies specifically aimed at reducing GHG emissions - alongside technology and other policies - have helped to weaken the link between GHG emissions and gross domestic product (GDP) (high confidence). In addition, in a large group of countries, fuel taxes (although not necessarily designed for the purpose of mitigation) have had effects that are akin to sectoral carbon taxes (robust evidence, medium agreement). Revenues from carbon taxes or auctioned emission allowances are used in some countries to reduce other taxes and/or to provide transfers to low-income groups. This illustrates the general principle that mitigation policies that raise government revenue generally have lower social costs than approaches which do not. \{WGIII SPM.5.1\}

Economic instruments in the form of subsidies may be applied across sectors, and include a variety of policy designs, such as tax rebates or exemptions, grants, loans and credit lines. An increasing number and variety of RE policies including subsidies-motivated by many factors - have driven escalated growth of RE technologies in recent years. Government policies play a crucial role in accelerating the deployment of RE technologies. Energy access and social and economic development have been the primary drivers in most developing countries whereas secure energy supply and environmental concerns have been most important in developed countries. The focus of policies is 
Table 4.7 | Sectoral Policy Instruments. \{WGIII Table 15.2\}

\begin{tabular}{|c|c|c|c|c|c|c|}
\hline $\begin{array}{l}\text { Policy } \\
\text { Instruments }\end{array}$ & Energy & Transport & Buildings & Industry & AFOLU & $\begin{array}{l}\text { Human Settlements } \\
\text { and Infrastructure }\end{array}$ \\
\hline $\begin{array}{l}\text { Economic } \\
\text { Instruments } \\
\text { - Taxes } \\
\text { (carbon taxes } \\
\text { may be } \\
\text { economy-wide) }\end{array}$ & $\begin{array}{l}\text { - Carbon tax (e.g., } \\
\text { applied to electricity } \\
\text { or fuels) }\end{array}$ & $\begin{array}{l}\text { - Fuel taxes } \\
\text { - Congestion charges, } \\
\text { vehicle registration } \\
\text { fees, road tolls } \\
\text { - Vehicle taxes }\end{array}$ & $\begin{array}{l}\text { - Carbon and/or } \\
\text { energy taxes (either } \\
\text { sectoral or } \\
\text { economy-wide) }\end{array}$ & $\begin{array}{l}\text { - Carbon tax or energy } \\
\text { tax } \\
\text { - Waste disposal taxes } \\
\text { or charges }\end{array}$ & $\begin{array}{l}\text { - Fertilizer or nitrogen } \\
\text { taxes to reduce } \\
\text { nitrous oxide }\left(\mathrm{N}_{2} \mathrm{O}\right)\end{array}$ & $\begin{array}{l}\text { - Sprawl taxes, Impact } \\
\text { fees, exactions, } \\
\text { split-rate property } \\
\text { taxes, tax increment } \\
\text { finance, betterment } \\
\text { taxes, congestion } \\
\text { charges }\end{array}$ \\
\hline $\begin{array}{l}\text { Economic } \\
\text { Instruments } \\
\text { - Tradable } \\
\text { Allowances } \\
\text { (may be } \\
\text { economy-wide) }\end{array}$ & $\begin{array}{l}\text { - } \quad \text { Emission trading } \\
\text { - Emission credits } \\
\text { under the Clean } \\
\text { Development } \\
\text { Mechanism (CDM) } \\
\text { - } \quad \text { Tradable Green } \\
\text { Certificates }\end{array}$ & $\begin{array}{l}\text { - Fuel and vehicle } \\
\text { standards }\end{array}$ & $\begin{array}{l}\text { Tradable certificates } \\
\text { for energy efficiency } \\
\text { improvements (white } \\
\text { certificates) }\end{array}$ & $\begin{array}{l}\text { - Emission trading } \\
\text { - Emission credits } \\
\text { under CDM } \\
\text { - Tradable Green } \\
\text { Certificates }\end{array}$ & $\begin{array}{l}\text { - Emission credits } \\
\text { under CDM } \\
\text { - Compliance schemes } \\
\text { outside Kyoto } \\
\text { protocol (national } \\
\text { schemes) } \\
\text { - Voluntary carbon } \\
\text { markets }\end{array}$ & $\begin{array}{l}\text { - Urban-scale cap and } \\
\text { trade }\end{array}$ \\
\hline $\begin{array}{l}\text { Economic } \\
\text { Instruments } \\
\text { - Subsidies }\end{array}$ & $\begin{array}{l}\text { - Fossil fuel subsidy } \\
\text { removal } \\
\text { - Feed in tariffs (FITs) } \\
\text { for renewable energy }\end{array}$ & $\begin{array}{l}\text { - Biofuel subsidies } \\
\text { - Vehicle purchase } \\
\text { subsidies } \\
\text { - Feebates }\end{array}$ & $\begin{array}{l}\text { - Subsidies or tax } \\
\text { exemptions for } \\
\text { investment in } \\
\text { efficient buildings, } \\
\text { retrofits and } \\
\text { products } \\
\text { - Subsidized loans }\end{array}$ & $\begin{array}{l}\text { - Subsidies (e.g., for } \\
\text { energy audits) } \\
\text { - Fiscal incentives (e.g., } \\
\text { for fuel switching) }\end{array}$ & $\begin{array}{l}\text { - Credit lines for } \\
\text { low-carbon } \\
\text { agriculture, } \\
\text { sustainable forestry }\end{array}$ & $\begin{array}{l}\text { - Special Improvement } \\
\text { or Redevelopment } \\
\text { Districts }\end{array}$ \\
\hline $\begin{array}{l}\text { Regulatory } \\
\text { Approaches }\end{array}$ & $\begin{array}{l}\text { - Efficiency or } \\
\text { environmental } \\
\text { performance } \\
\text { standards } \\
\text { - Renewable Portfolio } \\
\text { Standards (RPS) for } \\
\text { renewable energy } \\
\text { (RE) } \\
\text { - Equitable access to } \\
\text { electricity grid } \\
\text { - Legal status of } \\
\text { long-term } \mathrm{CO}_{2} \\
\text { storage }\end{array}$ & $\begin{array}{l}\text { - Fuel economy } \\
\text { performance } \\
\text { standards } \\
\text { - Fuel quality } \\
\text { standards } \\
\text { - Greenhouse gas } \\
\text { (GHG) emission } \\
\text { performance } \\
\text { standards } \\
\text { - Regulatory } \\
\text { restrictions to } \\
\text { encourage modal } \\
\text { shifts (road to rail) } \\
\text { - Restriction on use of } \\
\text { vehicles in certain } \\
\text { areas } \\
\text { - Environmental } \\
\text { capacity constraints } \\
\text { on airports } \\
\text { - Urban planning and } \\
\text { zoning restrictions }\end{array}$ & $\begin{array}{l}\text { - } \text { Building codes and } \\
\text { standards } \\
\text { - } \text { Equipment and } \\
\text { appliance standards } \\
\text { - } \\
\text { Mandates for energy } \\
\text { retailers to assist } \\
\text { customers invest in } \\
\text { energy efficiency }\end{array}$ & $\begin{array}{l}\text { - Energy efficiency } \\
\text { standards for } \\
\text { equipment } \\
\text { - Energy management } \\
\text { systems (also } \\
\text { voluntary) } \\
\text { - Voluntary } \\
\text { agreements (where } \\
\text { bound by regulation) } \\
\text { - Labelling and public } \\
\text { procurement } \\
\text { regulations }\end{array}$ & $\begin{array}{l}\text { - National policies to } \\
\text { support REDD+ } \\
\text { including monitoring, } \\
\text { reporting and } \\
\text { verification } \\
\text { - Forest laws to reduce } \\
\text { deforestation } \\
\text { - Air and water } \\
\text { pollution control GHG } \\
\text { precursors } \\
\text { - Land use planning } \\
\text { and governance }\end{array}$ & $\begin{array}{l}\text { - Mixed use zoning } \\
\text { - Development } \\
\text { restrictions } \\
\text { - Affordable housing } \\
\text { mandates } \\
\text { - Site access controls } \\
\text { - Transfer development } \\
\text { rights } \\
\text { - Design codes } \\
\text { - Building codes } \\
\text { - Street codes } \\
\text { - Design standards }\end{array}$ \\
\hline $\begin{array}{l}\text { Information } \\
\text { Programmes }\end{array}$ & & $\begin{array}{l}\text { - Fuel labelling } \\
\text { - Vehicle efficiency } \\
\text { labelling }\end{array}$ & $\begin{array}{l}\text { - Energy audits } \\
\text { - Labelling } \\
\text { programmes } \\
\text { - Energy advice } \\
\text { programmes }\end{array}$ & $\begin{array}{l}\text { - Energy audits } \\
\text { - } \text { Benchmarking } \\
\text { - } \text { Brokerage for } \\
\text { industrial } \\
\text { cooperation }\end{array}$ & $\begin{array}{l}\text { - Certification schemes } \\
\text { for sustainable forest } \\
\text { practices } \\
\text { - Information policies } \\
\text { to support REDD+ } \\
\text { including monitoring, } \\
\text { reporting and } \\
\text { verification }\end{array}$ & \\
\hline $\begin{array}{l}\text { Government } \\
\text { Provision of } \\
\text { Public Goods or } \\
\text { Services }\end{array}$ & $\begin{array}{l}\text { - Research and } \\
\text { development } \\
\text { - Infrastructure } \\
\text { expansion (district } \\
\text { heating/cooling or } \\
\text { common carrier) }\end{array}$ & $\begin{array}{l}\text { - Investment in transit } \\
\text { and human powered } \\
\text { transport } \\
\text { - Investment in } \\
\text { alternative fuel } \\
\text { infrastructure } \\
\text { - Low-emission vehicle } \\
\text { procurement }\end{array}$ & $\begin{array}{l}\text { - Public procurement } \\
\text { of efficient buildings } \\
\text { and appliances }\end{array}$ & $\begin{array}{l}\text { - Training and } \\
\text { education } \\
\text { - Brokerage for } \\
\text { industrial } \\
\text { cooperation }\end{array}$ & $\begin{array}{l}\text { - Protection of } \\
\text { national, state, and } \\
\text { local forests. } \\
\text { - Investment in } \\
\text { improvement and } \\
\text { diffusion of } \\
\text { innovative } \\
\text { technologies in } \\
\text { agriculture and } \\
\text { forestry }\end{array}$ & $\begin{array}{l}\text { - Provision of utility } \\
\text { infrastructure, such } \\
\text { as electricity } \\
\text { distribution, district } \\
\text { heating/cooling and } \\
\text { wastewater } \\
\text { connections, etc. } \\
\text { - Park improvements } \\
\text { - Trail improvements } \\
\text { - Urban rail }\end{array}$ \\
\hline $\begin{array}{l}\text { Voluntary } \\
\text { Actions }\end{array}$ & & & $\begin{array}{l}\text { - } \text { Labelling } \\
\text { programmes for } \\
\text { efficient buildings } \\
\text { - }\end{array}$ & $\begin{array}{l}\text { - Voluntary agreements } \\
\text { on energy targets, } \\
\text { adoption of energy } \\
\text { management systems, } \\
\text { or resource efficiency }\end{array}$ & $\begin{array}{l}\text { - Promotion of } \\
\text { sustainability by } \\
\text { developing standards } \\
\text { and educational } \\
\text { campaigns }\end{array}$ & \\
\hline
\end{tabular}


broadening from a concentration primarily on RE electricity to include RE heating and cooling and transportation. \{SRREN SPM.7\}

The reduction of subsidies for GHG-related activities in various sectors can achieve emission reductions, depending on the social and economic context (high confidence). While subsidies can affect emissions in many sectors, most of the recent literature has focused on subsidies for fossil fuels. Since AR4 a small but growing literature based on economy-wide models has projected that complete removal of subsidies to fossil fuels in all countries could result in reductions in global aggregate emissions by mid-century (medium evidence, medium agreement). Studies vary in methodology, the type and definition of subsidies and the time frame for phase out considered. In particular, the studies assess the impacts of complete removal of all fossil fuel subsides without seeking to assess which subsidies are wasteful and inefficient, keeping in mind national circumstances. \{WGIII SPM.5.1\}

Regulatory approaches and information measures are widely used and are often environmentally effective (medium evidence, medium agreement). Examples of regulatory approaches include energy efficiency standards; examples of information programmes include labelling programmes that can help consumers make better-informed decisions. \{WGIII SPM.5.1\}

Mitigation policy could devalue fossil fuel assets and reduce revenues for fossil fuel exporters, but differences between regions and fuels exist (high confidence). Most mitigation scenarios are associated with reduced revenues from coal and oil trade for major exporters. The effect on natural gas export revenues is more uncertain. The availability of CCS would reduce the adverse effect of mitigation on the value of fossil fuel assets (medium confidence). \{WGIII SPM.5.1\}

Interactions between or among mitigation policies may be synergistic or may have no additive effect on reducing emissions (medium evidence, high agreement). For instance, a carbon tax can have an additive environmental effect to policies such as subsidies for the supply of RE. By contrast, if a cap and trade system has a sufficiently stringent cap to affect emission-related decisions, then other policies have no further impact on reducing emissions (although they may affect costs and possibly the viability of more stringent future targets) (medium evidence, high agreement). In either case, additional policies may be needed to address market failures relating to innovation and technology diffusion. \{WGIII SPM.5.1\}

Sub-national climate policies are increasingly prevalent, both in countries with national policies and in those without. These policies include state and provincial climate plans combining market, regulatory and information instruments, and sub-national cap-and-trade systems. In addition, transnational cooperation has arisen among sub-national actors, notably among institutional investors, NGOs seeking to govern carbon offset markets, and networks of cities seeking to collaborate in generating low-carbon urban development. \{WGIII 13.5.2, 15.2.4, 15.8\}

Co-benefits and adverse side effects of mitigation could affect achievement of other objectives such as those related to human health, food security, biodiversity, local environmental quality, energy access, livelihoods and equitable sustainable development: \{WGIII SPM.2\}

- Mitigation scenarios reaching about 450 or $500 \mathrm{ppm} \mathrm{CO}_{2}$-equivalent by 2100 show reduced costs for achieving air quality and energy security objectives, with significant co-benefits for human health, ecosystem impacts and sufficiency of resources and resilience of the energy system. \{WGIII SPM.4.1\}

- Some mitigation policies raise the prices for some energy services and could hamper the ability of societies to expand access to modern energy services to underserved populations (low confidence). These potential adverse side effects can be avoided with the adoption of complementary policies such as income tax rebates or other benefit transfer mechanisms (medium confidence). The costs of achieving nearly universal access to electricity and clean fuels for cooking and heating are projected to be between USD 72 to 95 billion per year until 2030 with minimal effects on GHG emissions (limited evidence, medium agreement) and multiple benefits in health and air pollutant reduction (high confidence). \{WGIII SPM.5.1\}

Whether or not side effects materialize, and to what extent side effects materialize, will be case- and site-specific, and depend on local circumstances and the scale, scope and pace of implementation. Many co-benefits and adverse side effects have not been well-quantified. \{WGIII SPM.4.1\}

\subsubsection{Technology development and transfer}

Technology policy (development, diffusion and transfer) complements other mitigation policies across all scales from international to sub-national, but worldwide investment in research in support of GHG mitigation is small relative to overall public research spending (high confidence). Technology policy includes technology-push (e.g., publicly-funded R\&D) and demand-pull (e.g., governmental procurement programmes). Such policies address a pervasive market failure because, in the absence of government policy such as patent protection, the invention of new technologies and practices from R\&D efforts has aspects of a public good and thus tends to be under-provided by market forces alone. Technology support policies have promoted substantial innovation and diffusion of new technologies, but the cost-effectiveness of such policies is often difficult to assess. Technology policy can increase incentives for participation and compliance with international cooperative efforts, particularly in the long run. \{WGIII SPM.5.1, 2.6.5, 3.11, 13.9, 13.12, 15.6.5\}

Many adaptation efforts also critically rely on diffusion and transfer of technologies and management practices, but their effective use depends on a suitable institutional, regulatory, social and cultural context (high confidence). Adaptation technologies are often familiar and already applied elsewhere. However, the success of technology transfer may involve not only the provision of finance and information, but also strengthening of policy and regulatory environments and capacities to absorb, employ and improve technologies appropriate to local circumstances. \{WGII 15.4\} 


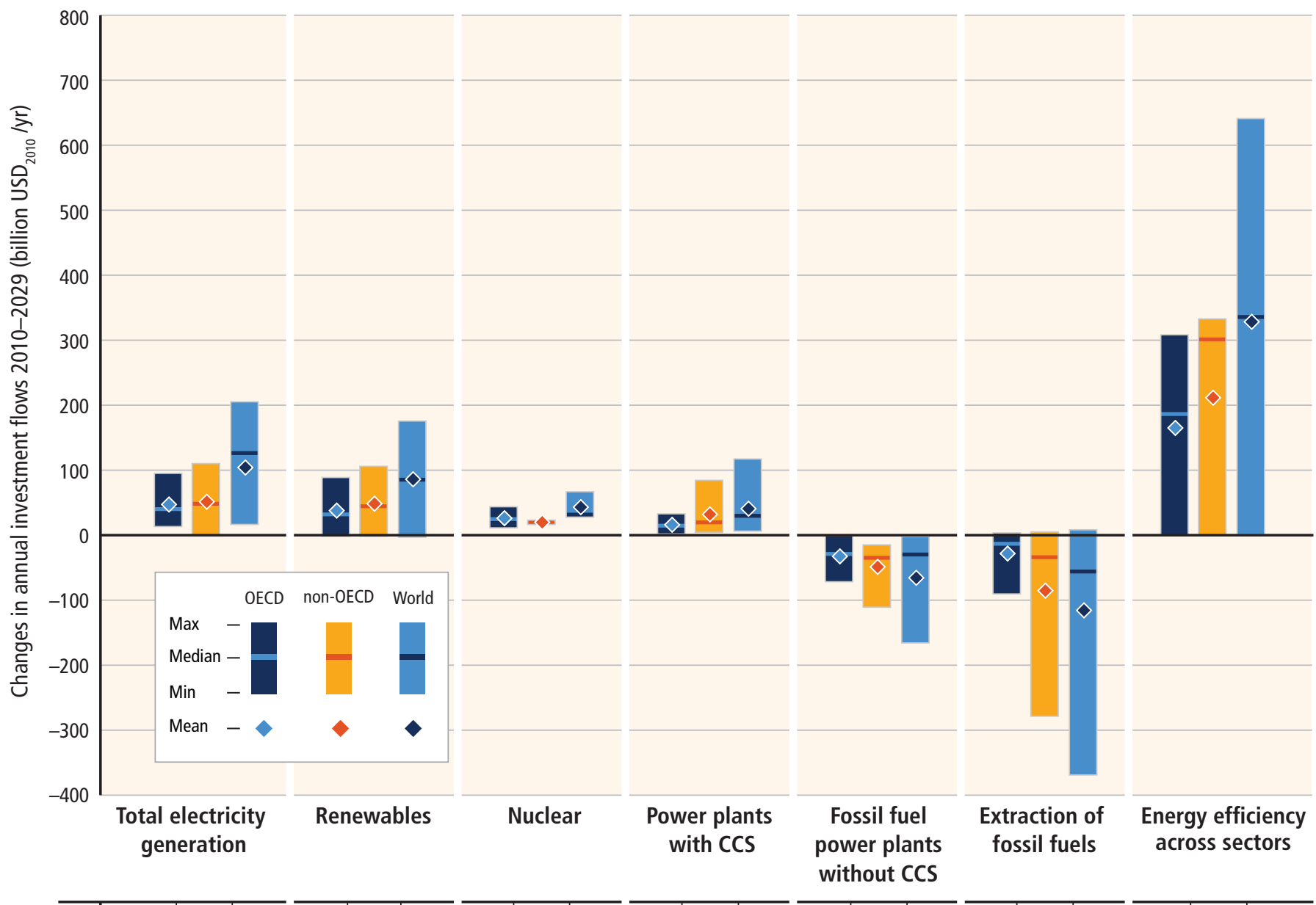

\begin{tabular}{l|l|l|l|l|l|l|l|l|l|l|l|l|l|l|l|l}
\hline $\mathrm{n}=$ & 4 & 4 & 5 & 4 & 4 & 5 & 4 & 4 & 5 & 4 & 4 & 5 & 4 & 4 & 5 & 4 \\
\hline
\end{tabular}

Figure 4.4 Change in annual investment flows from the average baseline level over the next two decades (2010 to 2029) for mitigation scenarios that stabilize concentrations (without overshoot) within the range of approximately 430 to $530 \mathrm{ppm} \mathrm{CO}_{2}$-eq by 2100. Total electricity generation (leftmost column) is the sum of renewable and nuclear energy, power plants with CCS, and fossil-fuel power plants without CCS. The vertical bars indicate the range between the minimum and maximum estimate; the horizontal bar indicates the median. The numbers in the bottom row show the total number of studies in the literature used in the assessment. Individual technologies shown are found to be used in different model scenarios in either a complementary or a synergistic way, depending largely on technology-specific assumptions and the timing and ambition level of the phase-in of global climate policies. \{WGIII Figure SPM.9\}

\subsubsection{Investment and finance}

Substantial reductions in emissions would require large changes in investment patterns (high confidence). Mitigation scenarios in which policies stabilize atmospheric concentrations (without overshoot) in the range from 430 to $530 \mathrm{ppm} \mathrm{CO}$-eq by $2100^{45}$ lead to substantial shifts in annual investment flows during the period 2010-2029 compared to baseline scenarios. Over the next two decades (20102029), annual investments in conventional fossil fuel technologies associated with the electricity supply sector are projected to decline in the scenarios by about USD 30 ( 2 to 166) billion (median: $-20 \%$ compared to 2010) while annual investment in low carbon electricity supply (i.e., renewables, nuclear and electricity with CCS) is projected to rise in the scenarios by about USD 147 (31 to 360) billion (median: $+100 \%$ compared to 2010) (limited evidence, medium agreement). In addition, annual incremental energy efficiency investments in transport, industry and buildings is projected to rise in the scenarios by about USD 336 (1 to 641) billion. Global total annual investment in the energy system is presently about USD 1,200 billion. This number includes only energy supply of electricity and heat and respective upstream and downstream activities. Energy efficiency investment or underlying sector investment is not included (Figure 4.4). \{WGIII SPM.5.1, 16.2\}

There is no widely agreed definition of what constitutes climate finance, but estimates of the financial flows associated with climate change mitigation and adaptation are available. See Figure 4.5 for an overview of climate finance flows. Published assessments of all current annual financial flows whose expected effect is to reduce net GHG emissions and/or to enhance resilience to climate change and climate variability show USD 343 to 385 billion per year

45 This range comprises scenarios that reach 430 to $480 \mathrm{ppm} \mathrm{CO}$-eq by 2100 (likely to limit warming to $2^{\circ} \mathrm{C}$ above pre-industrial levels) and scenarios that reach 480 to 530 ppm $\mathrm{CO}_{2}$-eq by 2100 (without overshoot: more likely than not to limit warming to $2^{\circ} \mathrm{C}$ above pre-industrial levels). 


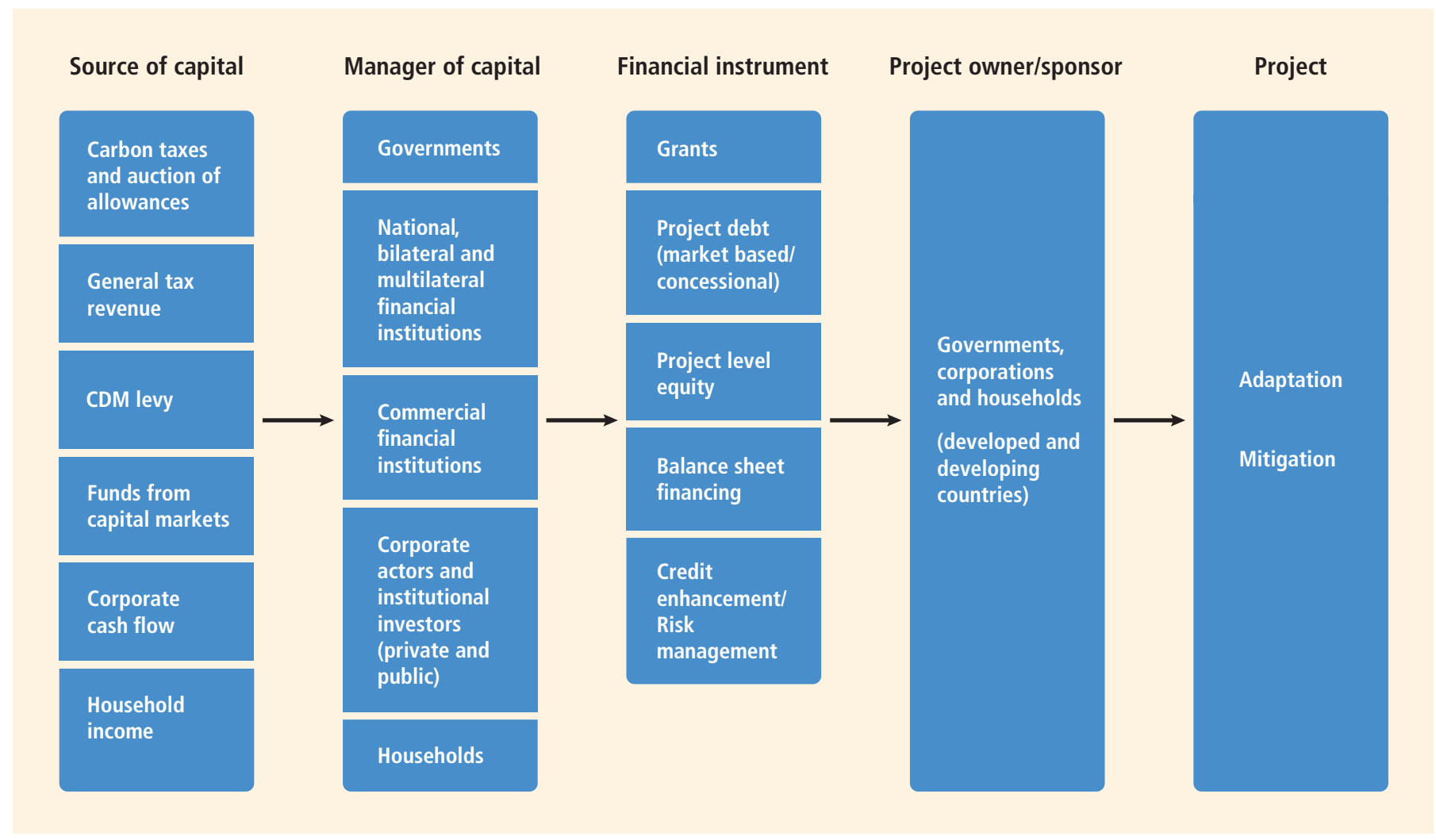

Figure 4.5 Overview of climate finance flows. Note: Capital should be understood to include all relevant financial flows. The size of the boxes is not related to the magnitude of the financial flow. \{WGIII Figure TS.40\}

globally (medium confidence). Out of this, total public climate finance that flowed to developing countries is estimated to be between USD 35 and 49 billion per year in 2011 and 2012 (medium confidence). Estimates of international private climate finance flowing to developing countries range from USD 10 to 72 billion per year including foreign direct investment as equity and loans in the range of USD 10 to 37 billion per year over the period of 2008-2011 (medium confidence). \{WGIII SPM.5.1\}

In many countries, the private sector plays central roles in the processes that lead to emissions as well as to mitigation and adaptation. Within appropriate enabling environments, the private sector, along with the public sector, can play an important role in financing mitigation and adaptation (medium evidence, high agreement). The share of total mitigation finance from the private sector, acknowledging data limitations, is estimated to be on average between two-thirds and three-fourths on the global level (2010-2012) (limited evidence, medium agreement). In many countries, public finance interventions by governments and international development banks encourage climate investments by the private sector and provide finance where private sector investment is limited. The quality of a country's enabling environment includes the effectiveness of its institutions, regulations and guidelines regarding the private sector, security of property rights, credibility of policies and other factors that have a substantial impact on whether private firms invest in new technologies and infrastructures. Dedicated policy instruments and financial arrangements, for example, credit insurance, feed-in tariffs, concessional finance or rebates provide an incentive for mitigation investment by improving the return adjusted for the risk for private actors. Public-private risk reduction initiatives (such as in the context of insurance systems) and economic diversification are examples of adaptation action enabling and relying on private sector participation. \{WGII SPM B-2, SPM C-1, WGIII SPM.5.1\}

Financial resources for adaptation have become available more slowly than for mitigation in both developed and developing countries. Limited evidence indicates that there is a gap between global adaptation needs and the funds available for adaptation (medium confidence). Potential synergies between international finance for disaster risk management and adaptation to climate change have not yet been fully realized (high confidence). There is a need for better assessment of global adaptation costs, funding and investment. Studies estimating the global cost of adaptation are characterized by shortcomings in data, methods and coverage (high confidence). \{WGII SPM C-1, 14.2, SREX SPM\} 


\subsection{Trade-offs, synergies and integrated responses}

There are many opportunities to link mitigation, adaptation and the pursuit of other societal objectives through integrated responses (high confidence). Successful implementation relies on relevant tools, suitable governance structures and enhanced capacity to respond (medium confidence).

A growing evidence base indicates close links between adaptation and mitigation, their co-benefits and adverse side effects, and recognizes sustainable development as the overarching context for climate policy (see Sections 3.5, 4.1, 4.2 and 4.3). Developing tools to address these linkages is critical to the success of climate policy in the context of sustainable development (see also Sections 4.4 and 3.5). This section presents examples of integrated responses in specific policy arenas, as well as some of the factors that promote or impede policies aimed at multiple objectives.

Increasing efforts to mitigate and adapt to climate change imply an increasing complexity of interactions, encompassing connections among human health, water, energy, land use and biodiversity (very high confidence). Mitigation can support the achievement of other societal goals, such as those related to human health, food security, environmental quality, energy access, livelihoods and sustainable development, although there can also be negative effects. Adaptation measures also have the potential to deliver mitigation co-benefits, and vice versa, and support other societal goals, though trade-offs can also arise. \{WGII SPM C-1, SPM C-2, 8.4, 9.3-9.4, 11.9, Box CC-WE, WGIII Table TS.3, Table TS.4, Table TS.5, Table TS.6, Table TS.7\}

Integration of adaptation and mitigation into planning and decision-making can create synergies with sustainable development (high confidence). Synergies and trade-offs among mitigation and adaptation policies and policies advancing other societal goals can be substantial, although sometimes difficult to quantify especially in welfare terms (see also Section 3.5). A multi-objective approach to policy-making can help manage these synergies and trade-offs. Policies advancing multiple goals may also attract greater support. \{WGII SPM C-1, SPM C-2, 20.3, WGIII 1.2.1, 3.6.3, 4.3, 4.6, 4.8, 6.6.1\}

Effective integrated responses depend on suitable tools and governance structures, as well as adequate capacity (medium confidence). Managing trade-offs and synergies is challenging and requires tools to help understand interactions and support decision-making at local and regional scales. Integrated responses also depend on governance that enables coordination across scales and sectors, supported by appropriate institutions. Developing and implementing suitable tools and governance structures often requires upgrading the human and institutional capacity to design and deploy integrated responses. \{WGII SPM C-1, SPM C-2, 2.2, 2.4, 15.4, 15.5, 16.3, Table 14-1, Table 16-1, WGIII TS.1, TS.3, 15.2\}
An integrated approach to energy planning and implementation that explicitly assesses the potential for co-benefits and the presence of adverse side effects can capture complementarities across multiple climate, social and environmental objectives (medium confidence). There are strong interactive effects across various energy policy objectives, such as energy security, air quality, health and energy access (see Figure 3.5) and between a range of social and environmental objectives and climate mitigation objectives (see Table 4.5). An integrated approach can be assisted by tools such as cost-benefit analysis, cost-effectiveness analysis, multi-criteria analysis and expected utility theory. It also requires appropriate coordinating institutions. \{WGIII Figure SPM.6, TS.1, TS.3\}

Explicit consideration of interactions among water, food, energy and biological carbon sequestration plays an important role in supporting effective decisions for climate resilient pathways (medium evidence, high agreement). Both biofuel-based power generation and large-scale afforestation designed to mitigate climate change can reduce catchment run-off, which may conflict with alternative water uses for food production, human consumption or the maintenance of ecosystem function and services (see also Box 3.4). Conversely, irrigation can increase the climate resilience of food and fibre production but reduces water availability for other uses. \{WGII Box CC-WE, Box TS.9\}

An integrated response to urbanization provides substantial opportunities for enhanced resilience, reduced emissions and more sustainable development (medium confidence). Urban areas account for more than half of global primary energy use and energy-related $\mathrm{CO}_{2}$ emissions (medium evidence, high agreement) and contain a high proportion of the population and economic activities at risk from climate change. In rapidly growing and urbanizing regions, mitigation strategies based on spatial planning and efficient infrastructure supply can avoid the lock-in of high emission patterns. Mixed-use zoning, transport-oriented development, increased density and co-located jobs and homes can reduce direct and indirect energy use across sectors. Compact development of urban spaces and intelligent densification can preserve land carbon stocks and land for agriculture and bioenergy. Reduced energy and water consumption in urban areas through greening cities and recycling water are examples of mitigation actions with adaptation benefits. Building resilient infrastructure systems can reduce vulnerability of urban settlements and cities to coastal flooding, sea level rise and other climate-induced stresses. [WGII SPM B-2, SPM C-1, TS B-2, TS C-1, TS C-2, WGIII SPM.4.2.5, TS.3\} 
Annexes 

ANNEX

User Guide 


\section{User Guide}

As defined in the IPCC Procedures, the Synthesis Report (SYR) synthesises and integrates material contained within IPCC Assessment Reports and Special Reports. The scope of the SYR of the Fifth Assessment Report (AR5) includes material contained in the three Working Group contributions to the AR5, and it draws on information contained in other IPCC Reports as required. The SYR is based exclusively on assessments by the IPCC

Working Groups; it does not refer to or assess the primary scientific literature itself.

The SYR is a self-contained, condensed summary of the much richer information contained in the underlying Working Group Reports. Users may wish to access relevant material at the required level of detail in the following manner: the report contains a Summary for Policymakers (SPM) that provides the most condensed summary of the current understanding of scientific, technical and socio-economic aspects of climate change. All references in curly brackets in this SPM refer to sections in the longer report. The longer report consists of an Introduction and four Topics. The numbers of the SPM sections largely correspond with the section numbers of the Topics. At the end of each paragraph, references are provided in italics between curly brackets. These refer to the Summaries for Policymakers (SPMs), Technical Summaries (TSs), Executive Summaries of chapters (ESs) and chapters (with chapter and section numbers) of the underlying Working Group contributions to the AR5 and Special Reports of the AR5. References to the IPCC Fourth Assessment Report (AR4) in 2007 are identified by adding "AR4" to the reference.

Users who wish to gain a better understanding of scientific details or access the primary scientific literature on which the SYR is based should refer to chapter sections of the underlying Working Group reports that are cited in the longer report of the SYR. The individual chapters of the Working Group reports provide references to the primary scientific literature on which IPCC assessments are based, and also offer the most detailed region- and sector-specific information.

A glossary, a list of acronyms, lists of authors and reviewers, a list of IPCC publications (annexes) and an index are provided to further facilitate the use of this report. 


\section{ANNEX

\section{Glossary Editors}

Katharine J. Mach (USA), Serge Planton (France), Christoph von Stechow (Germany)

\section{Glossary Contributors}

Myles R. Allen (United Kingdom), John Broome (United Kingdom), John A. Church (Australia), Leon Clarke (USA), Piers Forster (United Kingdom), Pierre Friedlingstein (United Kingdom/Belgium), Jan Fuglestvedt (Norway), Gabriele Hegerl (United Kingdom/Germany), Blanca Jiménez Cisneros (Mexico/UNESCO), Vladimir Kattsov (Russian Federation), Howard Kunreuther (USA), Leo Meyer (The Netherlands), Jan Minx (Germany), Yacob Mulugetta (Ethiopia), Karen O'Brien (Norway), Michael Oppenheimer (USA), Gian-Kasper Plattner (Switzerland), Andy Reisinger (New Zealand), Robert Scholes (South Africa), Melinda Tignor (Switzerland/USA), Detlef van Vuuren (The Netherlands)

\section{TSU Facilitation}

Noëmie Leprince-Ringuet (France)

This annex should be cited as:

IPCC, 2014: Annex II: Glossary [Mach, K.J., S. Planton and C. von Stechow (eds.)]. In: Climate Change 2014: Synthesis Report. Contribution of Working Groups I, II and III to the Fifth Assessment Report of the Intergovernmental Panel on Climate Change [Core Writing Team, R.K. Pachauri and L.A. Meyer (eds.)]. IPCC, Geneva, Switzerland, pp. 117-130. 
This glossary defines some specific terms as the Core Writing Team of the Synthesis Report intends them to be interpreted in the context of this report. Red, italicized words indicate that the term is defined in the glossary. The references to Working Groups (WG) I, II and III in italics at the end of each term in this glossary refer to the AR5 WG glossaries and should be read as: WGI (IPCC, 2013a), WGII (IPCC, 2014a), and WGIII (IPCC, 2014b).

\section{Abrupt change/abrupt climate change}

Abrupt change refers to a change that is substantially faster than the rate of change in the recent history of the affected components of a system. Abrupt climate change refers to a large-scale change in the climate system that takes place over a few decades or less, persists (or is anticipated to persist) for at least a few decades and causes substantial disruptions in human and natural systems. $\{W G I, I I, I I I\}$

\section{Adaptation}

The process of adjustment to actual or expected climate and its effects. In human systems, adaptation seeks to moderate or avoid harm or exploit beneficial opportunities. In some natural systems, human intervention may facilitate adjustment to expected climate and its effects ${ }^{1}$. $\{W G I I, I I I\}$

\section{Adaptation deficit}

The gap between the current state of a system and a state that minimizes adverse impacts from existing climate conditions and variability. $\{W G I I\}$

\section{Adaptation limit}

The point at which an actor's objectives (or system needs) cannot be secured from intolerable risks through adaptive actions. \{WGII\}

\section{Hard adaptation limit}

No adaptive actions are possible to avoid intolerable risks.

\section{Soft adaptation limit}

Options are currently not available to avoid intolerable risks through adaptive action.

\section{Adaptive capacity}

The ability of systems, institutions, humans and other organisms to adjust to potential damage, to take advantage of opportunities, or to respond to consequences ${ }^{2}$. $\{W G I I, I I I\}$

\section{Adverse side effects}

The negative effects that a policy or measure aimed at one objective might have on other objectives, irrespective of the net effect on overall social welfare. Adverse side effects are often subject to uncertainty and depend on local circumstances and implementation practices, among other factors. See also Co-benefits and Risk. $\{W G I I I\}$

\section{Afforestation}

Planting of new forests on lands that historically have not contained forests. For a discussion of the term forest and related terms such as afforestation, reforestation and deforestation, see the IPCC Special Report on Land Use, Land-Use Change, and Forestry (IPCC, 2000b). See also information provided by the United Nations Framework Convention on Climate Change (UNFCCC, 2013) and the report on Definitions and Methodological Options to Inventory Emissions from Direct Human-induced Degradation of Forests and Devegetation of Other Vegetation Types (IPCC, 2003). \{WGI, III\}

\section{Agriculture, Forestry and Other Land Use (AFOLU and FOLU/ LULUCF)}

AFOLU plays a central role for food security and sustainable development. The main mitigation options within AFOLU involve one or more of three strategies: prevention of emissions to the atmosphere by conserving existing carbon pools in soils or vegetation or by reducing emissions of methane and nitrous oxide; sequestration-increasing the size of existing carbon pools and thereby extracting carbon dioxide $\left(\mathrm{CO}_{2}\right)$ from the atmosphere; and substitution—substituting biological products for fossil fuels or energy-intensive products, thereby reducing $\mathrm{CO}_{2}$ emissions. Demand-side measures (e.g., reducing losses and wastes of food, changes in human diet, or changes in wood consumption) may also play a role.

FOLU (Forestry and Other Land Use) -also referred to as LULUCF (Land Use, Land-Use Change, and Forestry) - is the subset of AFOLU emissions and removals of greenhouse gases (GHGs) resulting from direct human-induced land use, land-use change, and forestry activities excluding agricultural emissions. \{WGIII\}

\section{Albedo}

The fraction of solar radiation reflected by a surface or object, often expressed as a percentage. Snow-covered surfaces have a high albedo, the albedo of soils ranges from high to low and vegetation-covered surfaces and oceans have a low albedo. The Earth's planetary albedo varies mainly through varying cloudiness, snow, ice, leaf area and land cover changes. $\{W G I, I I I\}$

\section{Altimetry}

A technique for measuring the height of the Earth's surface with respect to the geocentre of the Earth within a defined terrestrial reference frame (geocentric sea level). $\{W G l\}$

\section{Ancillary benefits}

See Co-benefits. \{WGIII, III\}

Attribution

See Detection and attribution. $\{W G I, I I\}$.

\section{Baseline/reference}

The baseline (or reference) is the state against which change is measured. A baseline period is the period relative to which anomalies are computed. In the context of transformation pathways, the term baseline

Reflecting progress in science, this glossary entry differs in breadth and focus from the entry used in the Fourth Assessment Report and other IPCC reports.

2 This glossary entry builds from definitions used in previous IPCC reports and the Millennium Ecosystem Assessment (MEA, 2005). 
scenarios refers to scenarios that are based on the assumption that no mitigation policies or measures will be implemented beyond those that are already in force and/or are legislated or planned to be adopted. Baseline scenarios are not intended to be predictions of the future, but rather counterfactual constructions that can serve to highlight the level of emissions that would occur without further policy effort. Typically, baseline scenarios are then compared to mitigation scenarios that are constructed to meet different goals for greenhouse gas (GHG) emissions, atmospheric concentrations or temperature change. The term baseline scenario is used interchangeably with reference scenario and no policy scenario. In much of the literature the term is also synonymous with the term business-as-usual (BAU) scenario, although the term $B A U$ has fallen out of favour because the idea of business as usual in century-long socio-economic projections is hard to fathom. See also Emission scenario, Representative Concentration Pathways (RCPS) and SRES scenarios. $\{W G I, I I, I I\}$

\section{Biodiversity}

The variability among living organisms from terrestrial, marine and other ecosystems. Biodiversity includes variability at the genetic, species and ecosystem levels ${ }^{3}$. $\{W G I I, I I\}$

\section{Bioenergy and Carbon Dioxide Capture and Storage (BECCS)} The application of Carbon Dioxide Capture and Storage (CCS) technology to bioenergy conversion processes. Depending on the total lifecycle emissions, including total marginal consequential effects (from indirect land-use change (iLUC) and other processes), BECCS has the potential for net carbon dioxide $\left(\mathrm{CO}_{2}\right)$ removal from the atmosphere. See also Sequestration. $\{W G I I I\}$

\section{Burden sharing/effort sharing}

In the context of mitigation, burden sharing refers to sharing the effort of reducing the sources or enhancing the sinks of greenhouse gases (GHGs) from historical or projected levels, usually allocated by some criteria, as well as sharing the cost burden across countries. \{WGIII\}

\section{Cancún Agreements}

A set of decisions adopted at the 16th Session of the Conference of the Parties (COP) to the United Nations Framework Convention on Climate Change (UNFCCC), including the following, among others: the newly established Green Climate Fund (GCF), a newly established technology mechanism, a process for advancing discussions on adaptation, a formal process for reporting mitigation commitments, a goal of limiting global mean surface temperature increase to $2^{\circ} \mathrm{C}$ and an agreement on MRV-Measurement, Reporting and Verification for those countries that receive international support for their mitigation efforts. $\{W G I I I\}$

\section{Cancún Pledges}

During 2010, many countries submitted their existing plans for controlling greenhouse gas (GHG) emissions to the Climate Change Secretariat and these proposals have now been formally acknowledged under the United Nations Framework Convention on Climate Change (UNFCCC). Developed countries presented their plans in the shape of economy-wide targets to reduce emissions, mainly up to 2020, while developing countries proposed ways to limit their growth of emissions in the shape of plans of action. $\{W G I I I\}$

\section{Carbon cycle}

The term used to describe the flow of carbon (in various forms, e.g., as carbon dioxide $\left(\mathrm{CO}_{2}\right)$ ) through the atmosphere, ocean, terrestrial and marine biosphere and lithosphere. In this report, the reference unit for the global carbon cycle is $\mathrm{GtCO}_{2}$ or $\mathrm{GtC}$ (Gigatonne of carbon $=1 \mathrm{GtC}$ $=10^{15}$ grams of carbon. This corresponds to $\left.3.667 \mathrm{GtCO}_{2}\right) .\{W G I, I I, I I I\}$

\section{Carbon Dioxide Capture and Storage (CCS)}

A process in which a relatively pure stream of carbon dioxide $\left(\mathrm{CO}_{2}\right)$ from industrial and energy-related sources is separated (captured), conditioned, compressed and transported to a storage location for longterm isolation from the atmosphere. See also Bioenergy and Carbon Dioxide Capture and Storage (BECCS) and Sequestration. \{WGIII\}

\section{Carbon Dioxide Removal (CDR)}

Carbon Dioxide Removal methods refer to a set of techniques that aim to remove $\mathrm{CO}_{2}$ directly from the atmosphere by either (1) increasing natural sinks for carbon or (2) using chemical engineering to remove the $\mathrm{CO}_{2}$, with the intent of reducing the atmospheric $\mathrm{CO}_{2}$ concentration. CDR methods involve the ocean, land and technical systems, including such methods as iron fertilization, large-scale afforestation and direct capture of $\mathrm{CO}_{2}$ from the atmosphere using engineered chemical means. Some CDR methods fall under the category of geoengineering, though this may not be the case for others, with the distinction being based on the magnitude, scale and impact of the particular CDR activities. The boundary between CDR and mitigation is not clear and there could be some overlap between the two given current definitions (IPCC, 2012b, p. 2). See also Solar Radiation Management (SRM). \{WGI, III $\}$

\section{Carbon intensity}

The amount of emissions of carbon dioxide $\left(\mathrm{CO}_{2}\right)$ released per unit of another variable such as Gross Domestic Product (GDP), output energy use or transport. $\{W G I I\}$

\section{Carbon price}

The price for avoided or released carbon dioxide $\left(\mathrm{CO}_{2}\right)$ or $\mathrm{CO}_{2}$-equivalent emissions. This may refer to the rate of a carbon tax, or the price of emission permits. In many models that are used to assess the economic costs of mitigation, carbon prices are used as a proxy to represent the level of effort in mitigation policies. \{WGIII\}

\section{Carbon tax}

A levy on the carbon content of fossil fuels. Because virtually all of the carbon in fossil fuels is ultimately emitted as carbon dioxide $\left(\mathrm{CO}_{2}\right)$, a carbon tax is equivalent to an emission tax on $\mathrm{CO}_{2}$ emissions. $\{W G I I l\}$

\section{Climate}

Climate in a narrow sense is usually defined as the average weather, or more rigorously, as the statistical description in terms of the mean and variability of relevant quantities over a period of time ranging from months to thousands or millions of years. The classical period for averaging these 
variables is 30 years, as defined by the World Meteorological Organization. The relevant quantities are most often surface variables such as temperature, precipitation and wind. Climate in a wider sense is the state, including a statistical description, of the climate system. $\{W G I, I I, I I\}$

\section{Climate change}

Climate change refers to a change in the state of the climate that can be identified (e.g., by using statistical tests) by changes in the mean and/or the variability of its properties and that persists for an extended period, typically decades or longer. Climate change may be due to natural internal processes or external forcings such as modulations of the solar cycles, volcanic eruptions and persistent anthropogenic changes in the composition of the atmosphere or in land use. Note that the Framework Convention on Climate Change (UNFCCC), in its Article 1 defines climate change as: 'a change of climate which is attributed directly or indirectly to human activity that alters the composition of the global atmosphere and which is in addition to natural climate variability observed over comparable time periods'. The UNFCCC thus makes a distinction between climate change attributable to human activities altering the atmospheric composition and climate variability attributable to natural causes. See also Detection and Attribution. $\{W G I, I I, I I\}$

\section{Climate extreme (extreme weather or climate event)}

See Extreme weather event. $\{W G I, I I\}$

\section{Climate feedback}

An interaction in which a perturbation in one climate quantity causes a change in a second and the change in the second quantity ultimately leads to an additional change in the first. A negative feedback is one in which the initial perturbation is weakened by the changes it causes; a positive feedback is one in which the initial perturbation is enhanced. In the Fifth Assessment Report, a somewhat narrower definition is often used in which the climate quantity that is perturbed is the global mean surface temperature, which in turn causes changes in the global radiation budget. In either case, the initial perturbation can either be externally forced or arise as part of internal variability. $\{W G I, I I, I I\}$

\section{Climate finance}

There is no agreed definition of climate finance. The term climate finance is applied both to the financial resources devoted to addressing climate change globally and to financial flows to developing countries to assist them in addressing climate change. The literature includes several concepts in these categories, among which the most commonly used include: $\{W G I I\}$

\section{Incremental costs}

The cost of capital of the incremental investment and the change of operating and maintenance costs for a mitigation or adaptation project in comparison to a reference project. It can be calculated as the difference of the net present values of the two projects.

\section{Incremental investment}

The extra capital required for the initial investment for a mitigation or adaptation project in comparison to a reference project.

\section{Total climate finance}

All financial flows whose expected effect is to reduce net greenhouse gas (GHG) emissions and/or to enhance resilience to the impacts of climate variability and the projected climate change. This covers private and public funds, domestic and international flows and expenditures for mitigation and adaptation to current climate variability as well as future climate change.

\section{Total climate finance flowing to developing countries}

The amount of the total climate finance invested in developing countries that comes from developed countries. This covers private and public funds.

\section{Private climate finance flowing to developing countries}

Finance and investment by private actors in/from developed countries for mitigation and adaptation activities in developing countries.

\section{Public climate finance flowing to developing countries}

Finance provided by developed countries' governments and bilateral institutions as well as by multilateral institutions for mitigation and adaptation activities in developing countries. Most of the funds provided are concessional loans and grants.

\section{Climate model (spectrum or hierarchy)}

A numerical representation of the climate system based on the physical, chemical and biological properties of its components, their interactions and feedback processes and accounting for some of its known properties. The climate system can be represented by models of varying complexity; that is, for any one component or combination of components a spectrum or hierarchy of models can be identified, differing in such aspects as the number of spatial dimensions, the extent to which physical, chemical or biological processes are explicitly represented, or the level at which empirical parametrizations are involved. Coupled Atmosphere-Ocean General Circulation Models (AOGCMs) provide a representation of the climate system that is near or at the most comprehensive end of the spectrum currently available. There is an evolution towards more complex models with interactive chemistry and biology. Climate models are applied as a research tool to study and simulate the climate and for operational purposes, including monthly, seasonal and interannual climate predictions. $\{W G I, I I, I I\}$

\section{Climate projection}

A climate projection is the simulated response of the climate system to a scenario of future emission or concentration of greenhouse gases (GHGs) and aerosols, generally derived using climate models. Climate projections are distinguished from climate predictions by their dependence on the emission/concentration/radiative forcing scenario used, which is in turn based on assumptions concerning, for example, future socio-economic and technological developments that may or may not be realized. $\{W G I, I I, I I\}$

\section{Climate-resilient pathways}

Iterative processes for managing change within complex systems in order to reduce disruptions and enhance opportunities associated with climate change. $\{$ WGII\}

\section{Climate response}

See Climate sensitivity. $\{W G I\}$

\section{Climate sensitivity}

In IPCC reports, equilibrium climate sensitivity (units: ${ }^{\circ} \mathrm{C}$ ) refers to the equilibrium (steady state) change in the annual global mean surface 
temperature following a doubling of the atmospheric equivalent carbon dioxide $\left(\mathrm{CO}_{2}\right)$ concentration. Owing to computational constraints, the equilibrium climate sensitivity in a climate model is sometimes estimated by running an atmospheric general circulation model coupled to a mixed-layer ocean model, because equilibrium climate sensitivity is largely determined by atmospheric processes. Efficient models can be run to equilibrium with a dynamic ocean. The climate sensitivity parameter (units: $\left.{ }^{\circ} \mathrm{C}\left(\mathrm{W} \mathrm{m}^{-2}\right)^{-1}\right)$ refers to the equilibrium change in the annual global mean surface temperature following a unit change in radiative forcing.

The effective climate sensitivity (units: ${ }^{\circ} \mathrm{C}$ ) is an estimate of the global mean surface temperature response to doubled $\mathrm{CO}_{2}$ concentration that is evaluated from model output or observations for evolving nonequilibrium conditions. It is a measure of the strengths of the climate feedbacks at a particular time and may vary with forcing history and climate state and therefore may differ from equilibrium climate sensitivity.

The transient climate response (units: ${ }^{\circ} \mathrm{C}$ ) is the change in the global mean surface temperature, averaged over a 20-year period, centered at the time of atmospheric $\mathrm{CO}_{2}$ doubling, in a climate mode/ simulation in which $\mathrm{CO}_{2}$ increases at $1 \% / \mathrm{yr}$. It is a measure of the strength and rapidity of the surface temperature response to greenhouse gas (GHG) forcing. $\{W G I, I I, I I I\}$

\section{Climate system}

The climate system is the highly complex system consisting of five major components: the atmosphere, the hydrosphere, the cryosphere, the lithosphere and the biosphere and the interactions between them. The climate system evolves in time under the influence of its own internal dynamics and because of external forcings such as volcanic eruptions, solar variations and anthropogenic forcings such as the changing composition of the atmosphere and land-use change. $\{\mathrm{WGI}, \mathrm{II}, \mathrm{III}\}$

\section{Climate variability}

Climate variability refers to variations in the mean state and other statistics (such as standard deviations, the occurrence of extremes, etc.) of the climate on all spatial and temporal scales beyond that of individual weather events. Variability may be due to natural internal processes within the climate system (internal variability), or to variations in natural or anthropogenic external forcing (external variability). See also Climate change. $\{W G I, I I, I I I\}$

\section{$\mathrm{CO}_{2}$-equivalent $\left(\mathrm{CO}_{2}\right.$-eq) concentration}

The concentration of carbon dioxide $\left(\mathrm{CO}_{2}\right)$ that would cause the same radiative forcing as a given mixture of $\mathrm{CO}_{2}$ and other forcing components. Those values may consider only greenhouse gases (GHGs), or a combination of $\mathrm{GHGs}$, aerosols and surface albedo change. $\mathrm{CO}_{2}$-equivalent concentration is a metric for comparing radiative forcing of a mix of different forcing components at a particular time but does not imply equivalence of the corresponding climate change responses nor future forcing. There is generally no connection between $\mathrm{CO}_{2}$-equivalent emissions and resulting $\mathrm{CO}_{2}$-equivalent concentrations. $\{\mathrm{WGI}, \mathrm{III}\}$

\section{$\mathrm{CO}_{2}$-equivalent $\left(\mathrm{CO}_{2}\right.$-eq) emission}

The amount of carbon dioxide $\left(\mathrm{CO}_{2}\right)$ emission that would cause the same integrated radiative forcing, over a given time horizon, as an emitted amount of a greenhouse gas (GHG) or a mixture of GHGs.
The $\mathrm{CO}_{2}$-equivalent emission is obtained by multiplying the emission of a GHG by its Global Warming Potential (GWP) for the given time horizon (see WGI Chapter 8, Table 8.A.1 and WGIII Annex II.9.1 for GWP values of the different GHGs used here). For a mix of GHGs it is obtained by summing the $\mathrm{CO}_{2}$-equivalent emissions of each gas. $\mathrm{CO}_{2}$-equivalent emission is a common scale for comparing emissions of different GHGs but does not imply equivalence of the corresponding climate change responses. There is generally no connection between $\mathrm{CO}_{2}$-equivalent emissions and resulting $\mathrm{CO}_{2}$-equivalent concentrations. $\{W G I, I I I\}$

\section{Co-benefits}

The positive effects that a policy or measure aimed at one objective might have on other objectives, irrespective of the net effect on overall social welfare. Co-benefits are often subject to uncertainty and depend on local circumstances and implementation practices, among other factors. Co-benefits are also referred to as ancillary benefits. \{WGII, III\}

\section{Confidence}

The validity of a finding based on the type, amount, quality and consistency of evidence (e.g., mechanistic understanding, theory, data, models, expert judgment) and on the degree of agreement. In this report, confidence is expressed qualitatively (Mastrandrea et al., 2010). See WGI AR5 Figure 1.11 for the levels of confidence; see WGI AR5 Table 1.2 for the list of likelihood qualifiers; see WGII AR5 Box 1-1. See also Uncertainty. $\{W G I, I I, I I\}$

\section{Cost-effectiveness}

A policy is more cost-effective if it achieves a given policy goal at lower cost. Integrated models approximate cost-effective solutions, unless they are specifically constrained to behave otherwise. Cost-effective mitigation scenarios are those based on a stylized implementation approach in which a single price on carbon dioxide $\left(\mathrm{CO}_{2}\right)$ and other greenhouse gases (GHGs) is applied across the globe in every sector of every country and that rises over time in a way that achieves lowest global discounted costs. $\{W G I I\}$

\section{Decarbonization}

The process by which countries or other entities aim to achieve a low-carbon economy, or by which individuals aim to reduce their consumption of carbon. $\{W G I I, I I\}$

\section{Deforestation}

Conversion of forest to non-forest. For a discussion of the term forest and related terms such as afforestation, reforestation and deforestation, see the IPCC Special Report on Land Use, Land-Use Change, and Forestry (IPCC, 2000b). See also information provided by the United Nations Framework Convention on Climate Change (UNFCCC, 2013) and the report on Definitions and Methodological Options to Inventory Emissions from Direct Human-induced Degradation of Forests and Devegetation of Other Vegetation Types (IPCC, 2003). \{WGI, II\}

\section{Detection and attribution}

Detection of change is defined as the process of demonstrating that climate or a system affected by climate has changed in some defined statistical sense, without providing a reason for that change. An identified change is detected in observations if its likelihood of occurrence by chance due to internal variability alone is determined to be small, 
for example, $<10 \%$. Attribution is defined as the process of evaluating the relative contributions of multiple causal factors to a change or event with an assignment of statistical confidence (Hegerl et al., 2010). $\{W G I, I I\}$

\section{Detection of impacts of climate change}

For a natural, human or managed system, identification of a change from a specified baseline. The baseline characterizes behavior in the absence of climate change and may be stationary or non-stationary (e.g., due to land-use change). \{WGII\}

\section{Disaster}

Severe alterations in the normal functioning of a community or a society due to hazardous physical events interacting with vulnerable social conditions, leading to widespread adverse human, material, economic or environmental effects that require immediate emergency response to satisfy critical human needs and that may require external support for recovery. \{WGII\}

\section{Discounting}

A mathematical operation making monetary (or other) amounts received or expended at different times (years) comparable across time. The discounter uses a fixed or possibly time-varying discount rate $(>0)$ from year to year that makes future value worth less today. $\{W G I I, I I I\}$

\section{Drought}

A period of abnormally dry weather long enough to cause a serious hydrological imbalance. Drought is a relative term; therefore any discussion in terms of precipitation deficit must refer to the particular precipitation-related activity that is under discussion. For example, shortage of precipitation during the growing season impinges on crop production or ecosystem function in general (due to soil moisture drought, also termed agricultural drought) and during the runoff and percolation season primarily affects water supplies (hydrological drought). Storage changes in soil moisture and groundwater are also affected by increases in actual evapotranspiration in addition to reductions in precipitation. A period with an abnormal precipitation deficit is defined as a meteorological drought. A megadrought is a very lengthy and pervasive drought, lasting much longer than normal, usually a decade or more. For the corresponding indices, see WGI AR5 Box 2.4 $\{W G I, I I\}$

\section{Early warning system}

The set of capacities needed to generate and disseminate timely and meaningful warning information to enable individuals, communities and organizations threatened by a hazard to prepare to act promptly and appropriately to reduce the possibility of harm or loss ${ }^{4}$. $\{W G I I\}$

\section{Earth System Model (ESM)}

A coupled atmosphere-ocean general circulation model in which a representation of the carbon cycle is included, allowing for interactive calculation of atmospheric $\mathrm{CO}_{2}$ or compatible emissions. Additional components (e.g., atmospheric chemistry, ice sheets, dynamic vegetation, nitrogen cycle, but also urban or crop models) may be included. See also Climate model. $\{W G I, I I\}$

\section{Ecosystem}

An ecosystem is a functional unit consisting of living organisms, their non-living environment and the interactions within and between them. The components included in a given ecosystem and its spatial boundaries depend on the purpose for which the ecosystem is defined: in some cases they are relatively sharp, while in others they are diffuse. Ecosystem boundaries can change over time. Ecosystems are nested within other ecosystems and their scale can range from very small to the entire biosphere. In the current era, most ecosystems either contain people as key organisms, or are influenced by the effects of human activities in their environment. $\{W G I, I I, I I I\}$

\section{Ecosystem services}

Ecological processes or functions having monetary or non-monetary value to individuals or society at large. These are frequently classified as (1) supporting services such as productivity or biodiversity maintenance, (2) provisioning services such as food, fiber or fish, (3) regulating services such as climate regulation or carbon sequestration and (4) cultural services such as tourism or spiritual and aesthetic appreciation. $\{W G I I, I I\}$

\section{El Niño-Southern Oscillation (ENSO)}

The term El Niño was initially used to describe a warm-water current that periodically flows along the coast of Ecuador and Peru, disrupting the local fishery. It has since become identified with a basin-wide warming of the tropical Pacific Ocean east of the dateline. This oceanic event is associated with a fluctuation of a global-scale tropical and subtropical surface pressure pattern called the Southern Oscillation. This coupled atmosphere-ocean phenomenon, with preferred time scales of two to about seven years, is known as the El Niño-Southern Oscillation (ENSO). It is often measured by the surface pressure anomaly difference between Tahiti and Darwin or the sea surface temperatures in the central and eastern equatorial Pacific. During an ENSO event, the prevailing trade winds weaken, reducing upwelling and altering ocean currents such that the sea surface temperatures warm, further weakening the trade winds. This event has a great impact on the wind, sea surface temperature and precipitation patterns in the tropical Pacific. It has climatic effects throughout the Pacific region and in many other parts of the world, through global teleconnections. The cold phase of ENSO is called La Niña. For the corresponding indices, see WGI AR5 Box 2.5. \{WGI, II\}

\section{Emission scenario}

A plausible representation of the future development of emissions of substances that are potentially radiatively active (e.g., greenhouse gases (GHGs), aerosols) based on a coherent and internally consistent set of assumptions about driving forces (such as demographic and socio-economic development, technological change, energy and land use) and their key relationships. Concentration scenarios, derived from emission scenarios, are used as input to a climate model to compute climate projections. In IPCC (1992) a set of emission scenarios was presented which were used as a basis for the climate projections in IPCC (1996). These emission scenarios are referred to as the IS92 scenarios. In the IPCC Special Report on Emissions Scenarios (IPCC, 2000a) emission scenarios, the so-called SRES scenarios, were published, some of

4 This glossary entry builds from the definitions used in UNISDR (2009) and IPCC (2012a). 
which were used, among others, as a basis for the climate projections presented in Chapters 9 to 11 of IPCC WGI TAR (IPCC, 2001a) and Chapters 10 and 11 of IPCC WGI AR4 (IPCC, 2007) as well as in the IPCC WGI AR5 (IPCC, 2013b). New emission scenarios for climate change, the four Representative Concentration Pathways, were developed for, but independently of, the present IPCC assessment. See also Baseline/reference, Mitigation scenario and Transformation pathway. $\{W G I, I I, I I\}$

\section{Energy access}

Access to clean, reliable and affordable energy services for cooking and heating, lighting, communications and productive uses (AGECC, 2010). $\{W G I I I\}$

\section{Energy intensity}

The ratio of energy use to economic or physical output. \{WGIII\}

\section{Energy security}

The goal of a given country, or the global community as a whole, to maintain an adequate, stable and predictable energy supply. Measures encompass safeguarding the sufficiency of energy resources to meet national energy demand at competitive and stable prices and the resilience of the energy supply; enabling development and deployment of technologies; building sufficient infrastructure to generate, store and transmit energy supplies and ensuring enforceable contracts of delivery. $\{W G I I I\}$

\section{Ensemble}

A collection of model simulations characterizing a climate prediction or projection. Differences in initial conditions and model formulation result in different evolutions of the modeled system and may give information on uncertainty associated with model error and error in initial conditions in the case of climate forecasts and on uncertainty associated with model error and with internally generated climate variability in the case of climate projections. $\{W G I, I I\}$

\section{Equilibrium climate sensitivity}

See Climate sensitivity. \{WGI\}

\section{Eutrophication}

Over-enrichment of water by nutrients such as nitrogen and phosphorus. It is one of the leading causes of water quality impairment. The two most acute symptoms of eutrophication are hypoxia (or oxygen depletion) and harmful algal blooms. \{WGII\}

\section{Exposure}

The presence of people, livelihoods, species or ecosystems, environmental functions, services, and resources, infrastructure, or economic, social, or cultural assets in places and settings that could be adversely affected. $\{W G I I\}$

\section{External forcing}

External forcing refers to a forcing agent outside the climate system causing a change in the climate system. Volcanic eruptions, solar variations and anthropogenic changes in the composition of the atmosphere and land-use change are external forcings. Orbital forcing is also an external forcing as the insolation changes with orbital parameters eccentricity, tilt and precession of the equinox. $\{W G I, I I\}$

\section{Extreme weather event}

An extreme weather event is an event that is rare at a particular place and time of year. Definitions of rare vary, but an extreme weather event would normally be as rare as or rarer than the 10th or 90th percentile of a probability density function estimated from observations. By definition, the characteristics of what is called extreme weather may vary from place to place in an absolute sense. When a pattern of extreme weather persists for some time, such as a season, it may be classed as an extreme climate event, especially if it yields an average or total that is itself extreme (e.g., drought or heavy rainfall over a season). $\{W G I, I I\}$

\section{Feedback \\ See Climate feedback. $\{W G I, I I\}$}

Flood

The overflowing of the normal confines of a stream or other body of water, or the accumulation of water over areas not normally submerged. Floods include river (fluvial) floods, flash floods, urban floods, pluvial floods, sewer floods, coastal floods and glacial lake outburst floods. $\{W G I I\}$

\section{Food security}

A state that prevails when people have secure access to sufficient amounts of safe and nutritious food for normal growth, development and an active and healthy life. $\{W G I I, I I\}$

\section{Forest}

A vegetation type dominated by trees. Many definitions of the term forest are in use throughout the world, reflecting wide differences in biogeophysical conditions, social structure and economics. For a discussion of the term forest and related terms such as afforestation, reforestation and deforestation, see the IPCC Special Report on Land Use, Land-Use Change, and Forestry (IPCC, 2000b). See also information provided by the United Nations Framework Convention on Climate Change (UNFCCC, 2013) and the Report on Definitions and Methodological Options to Inventory Emissions from Direct Human-induced Degradation of Forests and Devegetation of Other Vegetation Types (IPCC, 2003). \{WGI, III $\}$

\section{Fuel poverty}

A condition in which a household is unable to guarantee a certain level of consumption of domestic energy services (especially heating) or suffers disproportionate expenditure burdens to meet these needs. $\{W G I I I\}$

\section{Geoengineering}

Geoengineering refers to a broad set of methods and technologies that aim to deliberately alter the climate system in order to alleviate the impacts of climate change. Most, but not all, methods seek to either (1) reduce the amount of absorbed solar energy in the climate system (Solar Radiation Management) or (2) increase net carbon sinks from the atmosphere at a scale sufficiently large to alter climate (Carbon Dioxide Removal). Scale and intent are of central importance. Two key characteristics of geoengineering methods of particular concern are that they use or affect the climate system (e.g., atmosphere, land or ocean) globally or regionally and/or could have substantive unintended effects that cross national boundaries. Geoengineering is different from weather modification and ecological engineering, but the boundary can be fuzzy (IPCC, 2012b, p. 2). \{WGI, II, III\} 
Global climate model (also referred to as general circulation model, both abbreviated as GCM)

See Climate model. $\{W G I, I I\}$

\section{Global Temperature change Potential (GTP)}

An index measuring the change in global mean surface temperature at a chosen point in time following an emission of a unit mass of a given substance, relative to that of the reference substance, carbon dioxide $\left(\mathrm{CO}_{2}\right)$. The Global Temperature change Potential (GTP) thus represents the combined effect of the differing times these substances remain in the atmosphere, their effectiveness in causing radiative forcing and the response of the climate system. The GTP has been defined in two different ways:

- Fixed GTP: based on a fixed time horizon in the future (such as GTP $_{100}$ for a time horizon of 100 years)

- Dynamic GTP: based on a target year (such as the year when global mean temperature is expected to reach a target level). In the dynamic GTP, the time horizon reduces over time as the target year is approached and hence the GTP value changes for emissions occurring further in the future. \{WGI Chapter 8\}

Global warming

Global warming refers to the gradual increase, observed or projected, in global surface temperature, as one of the consequences of radiative forcing caused by anthropogenic emissions. \{WGIII\}

\section{Global Warming Potential (GWP)}

An index measuring the radiative forcing following an emission of a unit mass of a given substance, accumulated over a chosen time horizon, relative to that of the reference substance, carbon dioxide $\left(\mathrm{CO}_{2}\right)$. The GWP thus represents the combined effect of the differing times these substances remain in the atmosphere and their effectiveness in causing radiative forcing. (WGI, III)

\section{Hazard}

The potential occurrence of a natural or human-induced physical event or trend or physical impact that may cause loss of life, injury, or other health impacts, as well as damage and loss to property, infrastructure, livelihoods, service provision, ecosystems and environmental resources. In this report, the term hazard usually refers to climate-related physical events or trends or their physical impacts. \{WGII\}

\section{Heat wave}

A period of abnormally and uncomfortably hot weather. $\{W G I, I I\}$

\section{Hydrological cycle}

The cycle in which water evaporates from the oceans and the land surface, is carried over the Earth in atmospheric circulation as water vapour, condenses to form clouds, precipitates over ocean and land as rain or snow, which on land can be intercepted by trees and vegetation, provides runoff on the land surface, infiltrates into soils, recharges groundwater, discharges into streams and ultimately flows out into the oceans, from which it will eventually evaporate again. The various systems involved in the hydrological cycle are usually referred to as hydrological systems. $\{W G I, I I\}$
Impacts (consequences, outcomes)

Effects on natural and human systems. In this report, the term impacts is used primarily to refer to the effects on natural and human systems of extreme weather and climate events and of climate change. Impacts generally refer to effects on lives, livelihoods, health, ecosystems, economies, societies, cultures, services and infrastructure due to the interaction of climate changes or hazardous climate events occurring within a specific time period and the vulnerability of an exposed society or system. Impacts are also referred to as consequences and outcomes. The impacts of climate change on geophysical systems, including floods, droughts and sea level rise, are a subset of impacts called physical impacts. \{WGII\}

\section{Indirect emissions}

Emissions that are a consequence of the activities within well-defined boundaries of, for instance, a region, an economic sector, a company or process, but which occur outside the specified boundaries. For example, emissions are described as indirect if they relate to the use of heat but physically arise outside the boundaries of the heat user, or to electricity production but physically arise outside of the boundaries of the power supply sector. $\{W G I I I\}$

\section{Industrial Revolution}

A period of rapid industrial growth with far-reaching social and economic consequences, beginning in Britain during the second half of the 18th century and spreading to Europe and later to other countries including the United States. The invention of the steam engine was an important trigger of this development. The industrial revolution marks the beginning of a strong increase in the use of fossil fuels and emission of, in particular, fossil carbon dioxide $\left(\mathrm{CO}_{2}\right)$. In this report the terms pre-industrial and industrial refer, somewhat arbitrarily, to the periods before and after 1750, respectively. $\{W G I, I I, I I I\}$

\section{Integrated assessment}

A method of analysis that combines results and models from the physical, biological, economic and social sciences and the interactions among these components in a consistent framework to evaluate the status and the consequences of environmental change and the policy responses to it. See also Integrated models. \{WGII, III\}

\section{Integrated Coastal Zone Management (ICZM)}

An integrated approach for sustainably managing coastal areas, taking into account all coastal habitats and uses. \{WGII\}

\section{Integrated models}

Integrated models explore the interactions between multiple sectors of the economy or components of particular systems, such as the energy system. In the context of transformation pathways, they refer to models that, at a minimum, include full and disaggregated representations of the energy system and its linkage to the overall economy that will allow for consideration of interactions among different elements of that system. Integrated models may also include representations of the full economy, land use and land-use change (LUC) and the climate system. See also Integrated assessment. \{WGIII\}

\section{Internal variability}

See Climate variability. $\{W G I\}$ 


\section{Irreversibility}

A perturbed state of a dynamical system is defined as irreversible on a given timescale, if the recovery timescale from this state due to natural processes is substantially longer than the time it takes for the system to reach this perturbed state. In the context of this report, the time scale of interest is centennial to millennial. See also Tipping point. $\{W G \mid\}$

\section{Land use and land-use change}

Land use refers to the total of arrangements, activities and inputs undertaken in a certain land cover type (a set of human actions). The term land use is also used in the sense of the social and economic purposes for which land is managed (e.g., grazing, timber extraction and conservation). In urban settlements it is related to land uses within cities and their hinterlands. Urban land use has implications on city management, structure and form and thus on energy demand, greenhouse gas (GHG) emissions and mobility, among other aspects. \{WGI, II, III\}

\section{Land-use change (LUC)}

Land-use change refers to a change in the use or management of land by humans, which may lead to a change in land cover. Land cover and land-use change may have an impact on the surface albedo, evapotranspiration, sources and sinks of greenhouse gases (GHGs), or other properties of the climate system and may thus give rise to radiative forcing and/or other impacts on climate, locally or globally. See also the IPCC Special Report on Land Use, Land-Use Change, and Forestry (IPCC, 2000b).

\section{Indirect land-use change (iLUC)}

Indirect land-use change refers to shifts in land use induced by a change in the production level of an agricultural product elsewhere, often mediated by markets or driven by policies. For example, if agricultural land is diverted to fuel production, forest clearance may occur elsewhere to replace the former agricultural production. See also Agriculture, Forestry and Other Land Use (AFOLU), Afforestation, Deforestation and Reforestation.

\section{Leakage}

Phenomena whereby the reduction in emissions (relative to a baseline) in a jurisdiction/sector associated with the implementation of mitigation policy is offset to some degree by an increase outside the jurisdiction/sector through induced changes in consumption, production, prices, land use and/or trade across the jurisdictions/sectors. Leakage can occur at a number of levels, be it a project, state, province, nation or world region.

In the context of Carbon Dioxide Capture and Storage (CCS), $\mathrm{CO}_{2}$ leakage refers to the escape of injected carbon dioxide $\left(\mathrm{CO}_{2}\right)$ from the storage location and eventual release to the atmosphere. In the context of other substances, the term is used more generically, such as for methane $\left(\mathrm{CH}_{4}\right)$ leakage (e.g., from fossil fuel extraction activities) and hydrofluorocarbon (HFC) leakage (e.g., from refrigeration and airconditioning systems). \{WGIII\}

\section{Likelihood}

The chance of a specific outcome occurring, where this might be estimated probabilistically. Likelihood is expressed in this report using a standard terminology (Mastrandrea et al., 2010), defined in WGI AR5
Table 1.2 and WGII AR5 Box 1-1. See also Confidence and Uncertainty. $\{W G I, I I, I I I\}$

\section{Lock-in}

Lock-in occurs when a market is stuck with a standard even though participants would be better off with an alternative. In this report, lock-in is used more broadly as path dependence, which is the generic situation where decisions, events or outcomes at one point in time constrain adaptation, mitigation or other actions or options at a later point in time. $\{W G I I, I I I\}$

\section{Low regrets policy}

A policy that would generate net social and/or economic benefits under current climate and a range of future climate change scenarios. $\{W G I l\}$

\section{Marine-based ice sheet}

An ice sheet containing a substantial region that rests on a bed lying below sea level and whose perimeter is in contact with the ocean. The best known example is the West Antarctic ice sheet. $\{W G \mid\}$

\section{Meridional Overturning Circulation (MOC)}

Meridional (north-south) overturning circulation in the ocean quantified by zonal (east-west) sums of mass transports in depth or density layers. In the North Atlantic, away from the subpolar regions, the MOC (which is in principle an observable quantity) is often identified with the thermohaline circulation (THC), which is a conceptual and incomplete interpretation. It must be borne in mind that the MOC is also driven by wind and can also include shallower overturning cells such as occur in the upper ocean in the tropics and subtropics, in which warm (light) waters moving poleward are transformed to slightly denser waters and subducted equatorward at deeper levels. $\{W G I, I I\}$

\section{Mitigation (of climate change)}

A human intervention to reduce the sources or enhance the sinks of greenhouse gases (GHGs). This report also assesses human interventions to reduce the sources of other substances which may contribute directly or indirectly to limiting climate change, including, for example, the reduction of particulate matter emissions that can directly alter the radiation balance (e.g., black carbon) or measures that control emissions of carbon monoxide, nitrogen oxides, Volatile Organic Compounds and other pollutants that can alter the concentration of tropospheric ozone which has an indirect effect on the climate. $\{W G I, I I, I I I\}$

\section{Mitigation scenario}

A plausible description of the future that describes how the (studied) system responds to the implementation of mitigation policies and measures. See also Baseline/reference, Emission scenario, Representative Concentration Pathways (RCPS), SRES scenarios and Transformation pathway. \{WGIII\}

\section{Net negative emissions}

A situation of net negative emissions is achieved when, as result of human activities, more greenhouse gases (GHGs) are sequestered or stored than are released into the atmosphere. \{SYR Box 2.2, footnote 29\}

\section{Ocean acidification}

Ocean acidification refers to a reduction in the $\mathrm{pH}$ of the ocean over an extended period, typically decades or longer, which is caused primarily 
by uptake of carbon dioxide $\left(\mathrm{CO}_{2}\right)$ from the atmosphere, but can also be caused by other chemical additions or subtractions from the ocean. Anthropogenic ocean acidification refers to the component of $\mathrm{pH}$ reduction that is caused by human activity (IPCC, 2011, p. 37). $\{W G I, I I\}$

\section{Overshoot pathways}

Emissions, concentration or temperature pathways in which the metric of interest temporarily exceeds, or overshoots the long-term goal. $\{W G I I I\}$

\section{Oxygen Minimum Zone (OMZ)}

The midwater layer (200-1000 m) in the open ocean in which oxygen saturation is the lowest in the ocean. The degree of oxygen depletion depends on the largely bacterial consumption of organic matter and the distribution of the OMZs is influenced by large-scale ocean circulation. In coastal oceans, OMZs extend to the shelves and may also affect benthic ecosystems. $\{W G I I\}$

\section{Permafrost}

Ground (soil or rock and included ice and organic material) that remains at or below $0^{\circ} \mathrm{C}$ for at least two consecutive years. $\{W G I, I I\}$

\section{$\mathrm{pH}$}

$\mathrm{pH}$ is a dimensionless measure of the acidity of water (or any solution) given by its concentration of hydrogen ions $\left(\mathrm{H}^{+}\right)$. $\mathrm{pH}$ is measured on a logarithmic scale where $\mathrm{pH}=-\log _{10}\left(\mathrm{H}^{+}\right)$. Thus, a $\mathrm{pH}$ decrease of 1 unit corresponds to a 10 -fold increase in the concentration of $\mathrm{H}^{+}$, or acidity. $\{W G \mid\}$

\section{Poverty}

Poverty is a complex concept with several definitions stemming from different schools of thought. It can refer to material circumstances (such as need, pattern of deprivation or limited resources), economic conditions (such as standard of living, inequality or economic position) and/or social relationships (such as social class, dependency, exclusion, lack of basic security or lack of entitlement). $\{W G I I\}$

\section{Pre-industrial}

See Industrial Revolution. $\{W G I, I I, I I\}$

\section{Private costs}

Private costs are carried by individuals, companies or other private entities that undertake an action, whereas social costs include additionally the external costs on the environment and on society as a whole. Quantitative estimates of both private and social costs may be incomplete, because of difficulties in measuring all relevant effects. $\{W G I I I\}$

\section{Projection}

A projection is a potential future evolution of a quantity or set of quantities, often computed with the aid of a model. Unlike predictions, projections are conditional on assumptions concerning, for example, future socio-economic and technological developments that may or may not be realized. See also Climate projection. $\{W G I, I I\}$

\section{Radiative forcing}

The strength of drivers is quantified as Radiative Forcing (RF) in units watts per square meter $\left(\mathrm{W} / \mathrm{m}^{2}\right)$ as in previous IPCC assessments. RF is the change in energy flux caused by a driver and is calculated at the tropopause or at the top of the atmosphere. $\{W G I\}$

\section{Reasons For Concern (RFCs)}

Elements of a classification framework, first developed in the IPCC Third Assessment Report (IPCC, 2001b), which aims to facilitate judgments about what level of climate change may be dangerous (in the language of Article 2 of the United Nations Framework Convention on Climate Change (UNFCCC)) by aggregating impacts, risks and vulnerabilities. $\{W G I I\}$

\section{Reducing Emissions from Deforestation and Forest Degradation (REDD)}

An effort to create financial value for the carbon stored in forests, offering incentives for developing countries to reduce emissions from forested lands and invest in low-carbon paths to sustainable development (SD). It is therefore a mechanism for mitigation that results from avoiding deforestation. REDD+ goes beyond reforestation and forest degradation and includes the role of conservation, sustainable management of forests and enhancement of forest carbon stocks. The concept was first introduced in 2005 in the 11th Session of the Conference of the Parties (COP) in Montreal and later given greater recognition in the 13th Session of the COP in 2007 at Bali and inclusion in the Bali Action Plan which called for 'policy approaches and positive incentives on issues relating to reducing emissions from deforestation and forest degradation in developing countries (REDD) and the role of conservation, sustainable management of forests and enhancement of forest carbon stock in developing countries'. Since then, support for REDD has increased and has slowly become a framework for action supported by a number of countries. $\{W G I I\}$

\section{Reforestation}

Planting of forests on lands that have previously contained forests but that have been converted to some other use. For a discussion of the term forest and related terms such as afforestation, reforestation and deforestation, see the IPCC Special Report on Land Use, Land-Use Change, and Forestry (IPCC, 2000b). See also information provided by the United Nations Framework Convention on Climate Change (UNFCCC, 2013). See also the Report on Definitions and Methodological Options to Inventory Emissions from Direct Human-induced Degradation of Forests and Devegetation of Other Vegetation Types (IPCC, 2003). $\{W G I, I I, I I I\}$

\section{Representative Concentration Pathways (RCPs)}

Scenarios that include time series of emissions and concentrations of the full suite of greenhouse gases (GHGs) and aerosols and chemically active gases, as well as land use/land cover (Moss et al., 2008). The word representative signifies that each RCP provides only one of many possible scenarios that would lead to the specific radiative forcing characteristics. The term pathway emphasizes that not only the long-term concentration levels are of interest, but also the trajectory taken over time to reach that outcome (Moss et al., 2010).

RCPs usually refer to the portion of the concentration pathway extending up to 2100, for which Integrated Assessment Models produced corresponding emission scenarios. Extended Concentration Pathways (ECPs) describe extensions of the RCPs from 2100 to 2500 that were 
calculated using simple rules generated by stakeholder consultations and do not represent fully consistent scenarios.

Four RCPs produced from Integrated Assessment Models were selected from the published literature and are used in the present IPCC Assessment as a basis for the climate predictions and projections presented in WGI AR5 Chapters 11 to 14 (IPCC, 2013b):

\section{RCP2.6}

One pathway where radiative forcing peaks at approximately $3 \mathrm{~W} / \mathrm{m}^{2}$ before 2100 and then declines (the corresponding ECP assuming constant emissions after 2100).

\section{$R C P 4.5$ and $R C P 6.0$}

Two intermediate stabilization pathways in which radiative forcing is stabilized at approximately $4.5 \mathrm{~W} / \mathrm{m}^{2}$ and $6.0 \mathrm{~W} / \mathrm{m}^{2}$ after 2100 (the corresponding ECPs assuming constant concentrations after 2150).

\section{RCP8.5}

One high pathway for which radiative forcing reaches $>8.5 \mathrm{~W} / \mathrm{m}^{2}$ by 2100 and continues to rise for some amount of time (the corresponding ECP assuming constant emissions after 2100 and constant concentrations after 2250).

For further description of future scenarios, see WGI AR5 Box 1.1. See also van Vuuren et al., 2011. \{WGI, II, III\}

\section{Resilience}

The capacity of social, economic and environmental systems to cope with a hazardous event or trend or disturbance, responding or reorganizing in ways that maintain their essential function, identity and structure, while also maintaining the capacity for adaptation, learning and transformation ${ }^{5}$. \{WGII, III\}

\section{Risk}

The potential for consequences where something of value is at stake and where the outcome is uncertain, recognizing the diversity of values. Risk is often represented as probability or likelihood of occurrence of hazardous events or trends multiplied by the impacts if these events or trends occur. In this report, the term risk is often used to refer to the potential, when the outcome is uncertain, for adverse consequences on lives, livelihoods, health, ecosystems and species, economic, social and cultural assets, services (including environmental services) and infrastructure. $\{W G I I, I I\}$

\section{Risk management}

The plans, actions or policies to reduce the likelihood and/or consequences of risks or to respond to consequences. \{WGII\}

\section{Sequestration}

The uptake (i.e., the addition of a substance of concern to a reservoir) of carbon containing substances, in particular carbon dioxide $\left(\mathrm{CO}_{2}\right)$, in terrestrial or marine reservoirs. Biological sequestration includes direct removal of $\mathrm{CO}_{2}$ from the atmosphere through land-use change (LUC), afforestation, reforestation, revegetation, carbon storage in landfills and practices that enhance soil carbon in agriculture (cropland management, grazing land management). In parts of the literature, but not in this report, (carbon) sequestration is used to refer to Carbon Dioxide Capture and Storage (CCS). \{WGIII\}

\section{Sink}

Any process, activity or mechanism that removes a greenhouse gas $(\mathrm{GHG})$, an aerosol or a precursor of a GHG or aerosol from the atmosphere. $\{W G I, I I, I I I\}$

\section{Social cost of carbon}

The net present value of climate damages (with harmful damages expressed as a positive number) from one more tonne of carbon in the form of carbon dioxide $\left(\mathrm{CO}_{2}\right)$, conditional on a global emissions trajectory over time. $\{W G I I, I I I\}$

\section{Social costs}

See Private costs. \{WGIII\}

\section{Solar Radiation Management (SRM)}

Solar Radiation Management refers to the intentional modification of the Earth's shortwave radiative budget with the aim to reduce climate change according to a given metric (e.g., surface temperature, precipitation, regional impacts, etc.). Artificial injection of stratospheric aerosols and cloud brightening are two examples of SRM techniques. Methods to modify some fast-responding elements of the long wave radiative budget (such as cirrus clouds), although not strictly speaking SRM, can be related to SRM. SRM techniques do not fall within the usual definitions of mitigation and adaptation (IPCC, 2012b, p. 2). See also Carbon Dioxide Removal (CDR) and Geoengineering. \{WGI, III\}

\section{SRES scenarios}

SRES scenarios are emission scenarios developed by IPCC (2000a) and used, among others, as a basis for some of the climate projections shown in Chapters 9 to 11 of IPCC WGI TAR (IPCC, 2001a), Chapters 10 and 11 of IPCC WGI AR4 (IPCC, 2007), as well as in the IPCC WGI AR5 (IPCC, 2013b). \{WGI, II, III\}

\section{Storm surge}

The temporary increase, at a particular locality, in the height of the sea due to extreme meteorological conditions (low atmospheric pressure and/or strong winds). The storm surge is defined as being the excess above the level expected from the tidal variation alone at that time and place. $\{W G I, I I\}$

\section{Structural change}

Changes, for example, in the relative share of gross domestic product (GDP) produced by the industrial, agricultural, or services sectors of an economy, or more generally, systems transformations whereby some components are either replaced or potentially substituted by other components. $\{W G I I I\}$

\section{Sustainability}

A dynamic process that guarantees the persistence of natural and human systems in an equitable manner. $\{W G I I, I I\}$ 
Sustainable development

Development that meets the needs of the present without compromising the ability of future generations to meet their own needs (WCED, 1987). $\{$ WGII, III\}

\section{Thermal expansion}

In connection with sea level, this refers to the increase in volume (and decrease in density) that results from warming water. A warming of the ocean leads to an expansion of the ocean volume and hence an increase in sea level. $\{W G I, I I\}$

\section{Tipping point}

A level of change in system properties beyond which a system reorganizes, often abruptly, and does not return to the initial state even if the drivers of the change are abated. For the climate system, it refers to a critical threshold when global or regional climate changes from one stable state to another stable state. The tipping point event may be irreversible. See also Irreversibility. $\{W G I, I I, I I I\}$

\section{Transformation}

A change in the fundamental attributes of natural and human systems. $\{W G I I\}$

\section{Transformation pathway}

The trajectory taken over time to meet different goals for greenhouse gas (GHG) emissions, atmospheric concentrations, or global mean surface temperature change that implies a set of economic, technological and behavioural changes. This can encompass changes in the way energy and infrastructure are used and produced, natural resources are managed and institutions are set up and in the pace and direction of technological change (TC). See also Baseline/reference, Emission scenario, Mitigation scenario, Representative Concentration Pathways (RCPS) and SRES scenarios. \{WGIII\}

Transient Climate Response to Cumulative $\mathrm{CO}_{2}$ Emissions (TCRE) The transient global average surface temperature change per unit cumulated $\mathrm{CO}_{2}$ emissions, usually $1000 \mathrm{PgC}$. TCRE combines both information on the airborne fraction of cumulated $\mathrm{CO}_{2}$ emissions (the fraction of the total $\mathrm{CO}_{2}$ emitted that remains in the atmosphere) and on the transient climate response (TCR). $\{W G I\}$

\section{Uncertainty}

A state of incomplete knowledge that can result from a lack of information or from disagreement about what is known or even knowable. It may have many types of sources, from imprecision in the data to ambiguously defined concepts or terminology, or uncertain projections of human behaviour. Uncertainty can therefore be represented by quantitative measures (e.g., a probability density function) or by qualitative statements (e.g., reflecting the judgment of a team of experts) (see Moss and Schneider, 2000; Manning et al., 2004; Mastrandrea et al., 2010). See also Confidence and Likelihood. \{WGI, II, III\}

\section{Vulnerability}

The propensity or predisposition to be adversely affected. Vulnerability encompasses a variety of concepts and elements including sensitivity or susceptibility to harm and lack of capacity to cope and adapt. \{WGII\}

\section{References}

AGECC, 2010: Energy for a Sustainable Future. United Nations Secretary General's Advisory Group on Energy and Climate (AGECC), New York, NY, USA, 24 pp.

Arctic Council, 2013: Glossary of terms. In: Arctic Resilience Interim Report 2013. Stockholm Environment Institute and Stockholm Resilience Centre, Stockholm Sweden, p.viii.

Hegerl, G. C., O. Hoegh-Guldberg, G. Casassa, M. P. Hoerling, R. S. Kovats, C. Parmesan, D. W. Pierce and P. A. Stott, 2010: Good practice guidance paper on detection and attribution related to anthropogenic climate change. In: Meeting Report of the Intergovernmental Panel on Climate Change Expert Meeting on Detection and Attribution of Anthropogenic Climate Change [Stocker T. F. , C. B. Field, D. Qin, V. Barros, G.-K. Plattner, M. Tignor, P. M. Midgley and K. L. Ebi (eds.)]. IPCC Working Group I Technical Support Unit, University of Bern, Bern, Switzerland, 8 pp.

Heywood, V. H. (ed.), 1995: The Global Biodiversity Assessment. United Nations Environment Programme, Cambridge University Press, Cambridge, United Kingdom, $1152 \mathrm{pp}$.

IPCC, 1992: Climate Change 1992: The Supplementary Report to the IPCC Scientific Assessment [Houghton, J. T., B. A. Callander and S. K. Varney (eds.)]. Cambridge University Press, Cambridge, United Kingdom and New York, NY, USA, 116 pp.

IPCC, 1996: Climate Change 1995: The Science of Climate Change. Contribution of Working Group I to the Second Assessment Report of the Intergovernmental
Panel on Climate Change [Houghton, J. T., L. G. Meira, A. Callander, N. Harris, A. Kattenberg and K. Maskell (eds.)]. Cambridge University Press, Cambridge, United Kingdom and New York, NY, USA, 572 pp.

IPCC, 2000a: Emissions Scenarios. Special Report of Working Group III of the Intergovernmental Panel on Climate Change [Nakićenović, N. and R. Swart (eds.)]. Cambridge University Press, Cambridge, United Kingdom and New York, NY USA, 599 pp.

IPCC, 2000b: Land Use, Land-Use Change, and Forestry. Special Report of the Intergovernmental Panel on Climate Change [Watson, R. T., I. R. Noble, B. Bolin, N. H. Ravindranath, D. J. Verardo and D. J. Dokken (eds.)]. Cambridge University Press, Cambridge, United Kingdom and New York, NY, USA, 377 pp.

IPCC, 2001a: Climate Change 2001: The Scientific Basis. Contribution of Working Group I to the Third Assessment Report of the Intergovernmental Panel on Climate Change [Houghton, J. T., Y. Ding, D.J. Griggs, M. Noquer, P. J. van der Linden, X. Dai, K. Maskell and C. A. Johnson (eds.)]. Cambridge University Press, Cambridge, United Kingdom and New York, NY, USA, 881 pp.

IPCC, 2001b: Climate Change 2001: Impacts, Adaptation, and Vulnerability. Contribution of Working Group II to the Third Assessment Report of the Intergovernmental Panel on Climate Change [McCarthy, J., 0. Canziani, N. Leary, D. Dokken and K. White (eds.)],Cambridge University Press, Cambridge, United Kingdom and New York, NY, USA, 1032 pp. 
IPCC, 2003: Definitions and Methodological Options to Inventory Emissions from Direct Human-Induced Degradation of Forests and Devegetation of Other Vegetation Types [Penman, J., M. Gytarsky, T. Hiraishi, T. Krug, D. Kruger, R. Pipatti, L. Buendia, K. Miwa, T. Ngara, K. Tanabe and F. Wagner (eds.)]. The Institute for Global Environmental Strategies (IGES), Japan, 32 pp.

IPCC, 2007: Climate Change 2007: The Physical Science Basis. Contribution of Working Group I to the Fourth Assessment Report of the Intergovernmental Panel on Climate Change. [Solomon, S., D. Qin, M. Manning, Z. Chen, M. Marquis, K. B. Averyt, M. Tignor and H. L. Miller (eds.)]. Cambridge University Press, Cambridge, United Kingdom and New York, NY, USA, 996 pp.

IPCC, 2011: Workshop Report of the Intergovernmental Panel on Climate Change Workshop on Impacts of Ocean Acidification on Marine Biology and Ecosystems [Field, C. B., V. Barros, T. F. Stocker, D. Qin, K. J. Mach, G.-K. Plattner, M. D. Mastrandrea, M. Tignor and K. L. Ebi (eds.)]. IPCC Working Group II Technical Support Unit, Carnegie Institution, Stanford, CA, USA, 164 pp.

IPCC, 2012a: Managing the Risks of Extreme Events and Disasters to Advance Climate Change Adaptation. A Special Report of Working Groups I and II of the Intergovernmental Panel on Climate Change [Field, C. B., V. Barros, T. F. Stocker, D. Qin, D. J. Dokken, K. L. Ebi, M. D. Mastrandrea, K. J. Mach, G.-K. Plattner, S. K. Allen, M. Tignor and P. M. Midgley (eds.)]. Cambridge University Press, Cambridge, UK and New York, NY, USA, 582 pp.

IPCC, 2012b: Meeting Report of the Intergovernmental Panel on Climate Change Expert Meeting on Geoengineering [Edenhofer, O., R. Pichs-Madruga, Y. Sokona, C. Field, V. Barros, T. F. Stocker, Q. Dahe, J. Minx, K. J. Mach, G.-K. Plattner, S. Schlömer, G. Hansen and M. Mastrandrea (eds.)]. IPCC Working Group III Technical Support Unit, Potsdam Institute for Climate Impact Research, Potsdam, Germany, 99 pp.

IPCC, 2013a: Annex III: Glossary [Planton, S. (ed.)]. In: Climate Change 2013: The Physical Science Basis. Contribution of Working Group I to the Fifth Assessment Report of the Intergovernmental Panel on Climate Change [Stocker, T. F., D. Qin, G.-K. Plattner, M. Tignor, S. K. Allen, J. Boschung, A. Nauels, Y. Xia, V. Bex and P. M. Midgley (eds.)]. Cambridge University Press, Cambridge, United Kingdom and New York, NY, USA, pp. 1447-1466, doi:10.1017/CB09781107415324.031.

IPCC, 2013b: Climate Change 2013: The Physical Science Basis. Contribution of Working Group I to the Fifth Assessment Report of the Intergovernmental Panel on Climate Change [Stocker, T. F., D. Qin, G.-K. Plattner, M. Tignor, S. K. Allen, J. Boschung, A. Nauels, Y. Xia, V. Bex and P. M. Midgley (eds.)]. Cambridge University Press, Cambridge, United Kingdom and New York, NY, USA, 1535 pp., doi:10.1017/СB09781107415324.

IPCC, 2014a: Annex II: Glossary [Agard, J., E. L. F. Schipper, J. Birkmann, M. Campos, C. Dubeux, Y. Nojiri, L. Olsson, B. Osman-Elasha, M. Pelling, M. J. Prather, M. G. Rivera-Ferre, O. C. Ruppel, A. Sallenger, K. R. Smith, A. L. St. Clair, K. J. Mach, M. D. Mastrandrea and T. E. Bilir (eds.)]. In: Climate Change 2014: Impacts, Adaptation, and Vulnerability. Part B: Regional Aspects. Contribution of Working Group II to the Fifth Assessment Report of the Intergovernmental Panel on Climate Change [Barros, V. R., C. B. Field, D. J. Dokken, M. D. Mastrandrea, K. J. Mach, T. E. Bilir, M. Chatterjee, K. L. Ebi, Y. O. Estrada, R. C. Genova, B. Girma, E. S. Kissel, A. N. Levy, S. MacCracken, P. R. Mastrandrea and L. L. White (eds.)]. Cambridge University Press, Cambridge, United Kingdom and New York, NY, USA, pp. 1757-1776.

IPCC, 2014b: Annex I: Glossary, Acronyms and Chemical Symbols [Allwood, J. M., V. Bosetti, N. K. Dubash, L. Gómez-Echeverri and C. von Stechow (eds.)]. In: Climate
Change 2014: Mitigation of Climate Change. Contribution of Working Group III to the Fifth Assessment Report of the Intergovernmental Panel on Climate Change [Edenhofer, O., R. Pichs-Madruga, Y. Sokona, E. Farahani, S. Kadner, K. Seyboth, A. Adler, I. Baum, S. Brunner, P. Eickemeier, B. Kriemann, J. Savolainen, S. Schlömer, C. von Stechow, T. Zwickel and J. C. Minx (eds.)]. Cambridge University Press, Cambridge, United Kingdom and New York, NY, USA, pp. 1251-1274.

Manning, M. R., M. Petit, D. Easterling, J. Murphy, A. Patwardhan, H.-H. Rogner, R. Swart and G. Yohe (eds.), 2004: IPCC Workshop on Describing Scientific Uncertainties in Climate Change to Support Analysis of Risk of Options. Workshop Report. Intergovernmental Panel on Climate Change, Geneva, Switzerland, 138 pp.

Mastrandrea, M. D., C. B. Field, T. F. Stocker, O. Edenhofer, K. L. Ebi, D. J. Frame, H. Held, E. Kriegler, K. J. Mach, P. R. Matschoss, G.-K. Plattner, G. W. Yohe and F. W. Zwiers, 2010: Guidance Note for Lead Authors of the IPCC Fifth Assessment Report on Consistent Treatment of Uncertainties. Intergovernmental Panel on Climate Change (IPCC), Geneva, Switzerland, 4 pp.

MEA, 2005: Appendix D: Glossary. In: Ecosystems and Human Well-being: Current States and Trends. Findings of the Condition and Trends Working Group, Vol.1 [Hassan, R., R. Scholes, and N. Ash (eds.)]. Millennium Ecosystem Assessment (MEA), Island Press, Washington, DC, USA, pp. 893-900.

Moss, R. and S. Schneider, 2000: Uncertainties in the IPCC TAR: Recommendations to Lead Authors for More Consistent Assessment and Reporting. In: IPCC Supporting Material: Guidance Papers on Cross Cutting Issues in the Third Assessment Report of the IPCC [Pachauri, R., T. Taniguchi and K. Tanaka (eds.)]. Intergovernmental Panel on Climate Change, Geneva, Switzerland, pp. 33-51.

Moss, R., M. Babiker, S. Brinkman, E. Calvo, T. Carter, J. Edmonds, I. Elgizouli, S. Emori, L. Erda, K. Hibbard, R. Jones, M. Kainuma, J. Kelleher, J. F. Lamarque, M. Manning, B. Matthews, J. Meehl, L. Meyer, J. Mitchell, N. Nakicenovic, B. O'Neill, R. Pichs, K. Riahi, S. Rose, P. Runci, R. Stouffer, D. van Vuuren, J. Weyant, T. Wilbanks, J. P. van Ypersele and M. Zurek, 2008: Towards new scenarios for analysis of emissions, climate change, impacts and response strategies. IPCC Expert Meeting Report, 19-21 September, 2007, Noordwijkerhout, Netherlands, Intergovernmental Panel on Climate Change (IPCC), Geneva, Switzerland, 132 pp.

Moss, R., J. A., Edmonds, K. A. Hibbard, M. R. Manning, S. K. Rose, D. P. van Vuuren, T. R. Carter, S. Emori, M. Kainuma, T. Kram, G. A. Meehl, J. F. B. Mitchell, N. Nakicenovic, K. Riahi, S. J. Smith, R. J. Stouffer, A. M. Thomson, J. P. Weyant and T. J. Wilbanks, 2010: The next generation of scenarios for climate change research and assessment. Nature, 463, 747-756.

UNFCCC, 2013: Reporting and accounting of LULUCF activities under the Kyoto Protocol. United Nations Framework Convention on Climatic Change (UNFCCC), Bonn, Germany. Available at: http://unfecc.int/methods/lulucf/items/4129.php

UNISDR, 2009: 2009 UNISDR Terminology on Disaster Risk Reduction. United Nations International Strategy for Disaster Reduction (UNISDR), United Nations, Geneva, Switzerland, $30 \mathrm{pp}$.

van Vuuren, D. P., J. Edmonds, M. Kainuma, K. Riahi, A. Thomson, K. Hibbard, G. C. Hurtt, T. Kram, V. Krey, J. F. Lamarque, T. Masui, M. Meinshausen, N. Nakicenovic, S.J. Smith and S.K. Rose, 2011: The Representative Concentration Pathways: an overview. Climatic Change, 109, pp. 5-31. 
WCED, 1987: Our Common Future. World Commission on Environment and Development (WCED), Oxford University Press, Oxford, UK, 300 pp. 


\section{ANNEX}

Acronyms, Chemical Symbols and Scientific Units 


\begin{tabular}{|c|c|}
\hline$\mu \mathrm{atm}$ & Microatmosphere \\
\hline AFOLU & Agriculture, Forestry and Other Land Use \\
\hline AMOC & Atlantic Meridional Overturning Circulation \\
\hline AR4 & Fourth Assessment Report \\
\hline AR5 & Fifth Assessment Report \\
\hline BAT & Best Available Technique \\
\hline BAU & Business As Usual \\
\hline BECCS & $\begin{array}{l}\text { Bioenergy with Carbon Dioxide } \\
\text { Capture and Storage }\end{array}$ \\
\hline CCS & Carbon Capture and Storage \\
\hline CDM & Clean Development Mechanism \\
\hline CDR & Carbon Dioxide Removal \\
\hline $\mathrm{CF}_{4}$ & Perfluoromethane \\
\hline $\mathrm{CH}_{4}$ & Methane \\
\hline CHP & Combined Heat and Power \\
\hline CMIP5 & Coupled Model Intercomparison Project Phase 5 \\
\hline $\mathrm{CO}_{2}$ & Carbon Dioxide \\
\hline $\mathrm{CO}_{2}$-eq & Carbon Dioxide Equivalent \\
\hline CSP & Concentrating Solar Power \\
\hline DC & Developing Country \\
\hline ECS & Equilibrium Climate Sensitivity \\
\hline
\end{tabular}

EDGAR Emission Database for Global Atmospheric Research

EJ Exajoule

EMIC Earth System Model of Intermediate Complexity

ENSO El Niño-Southern Oscillation

ES Executive Summary

ESM Earth System Model

ETS Emissions Trading System

F-gases Fluorinated gases

FAQ Frequently Asked Question
FAR

FIT

FOLU Forestry and Other Land Use

GCM Global Climate Model

GDP Gross Domestic Product

GHG Greenhouse Gas

GMI Global Methane Initiative

Gt Gigatonnes

GTP Global Temperature change Potential

GWP Global Warming Potential

$\mathbf{H}_{2} \quad$ Hydrogen

HadCRUT4 Hadley Centre Climatic Research

Unit Gridded Surface Temperature Data Set 4

HDV Heavy-Duty Vehicles

HFC Hydrofluorocarbon

HFC-152a Hydrofluorocarbon-152a,

Difluoroethane

IAM Integrated Assessment Model

ICAO International Civil Aviation Organization

IMO International Maritime Organization

I0 International Organization

LDV Light-Duty Vehicles

LULUCF Land Use, Land-Use Change and Forestry

MAGICC Model for the Assessment of Greenhouse Gas Induced Climate Change

MEF Major Economies Forum

MRV Monitoring, Reporting and Verification

$\mathrm{N}_{2} \mathrm{O} \quad$ Nitrous Oxide

NAMA Nationally Appropriate Mitigation Action

NAP National Adaptation Plan

NAPA National Adaptation Programmes of Action 


\begin{tabular}{|c|c|}
\hline NGO & Non-Governmental Organization \\
\hline $\mathrm{O}_{2}$ & Oxygen \\
\hline OA & Ocean Acidification \\
\hline OECD & $\begin{array}{l}\text { Organisation for Economic Co-operation } \\
\text { and Development }\end{array}$ \\
\hline PFC & Perfluorocarbon \\
\hline ppb & parts per billion \\
\hline ppm & parts per million \\
\hline PV & Photovoltaic \\
\hline R\&D & Research and Development \\
\hline RCP & Representative Concentration Pathway \\
\hline RE & Renewable Energy \\
\hline REDD & $\begin{array}{l}\text { Reducing Emissions from Deforestation } \\
\text { and Forest Degradation }\end{array}$ \\
\hline REEEP & Renewable Energy and Energy Efficiency Partnership \\
\hline RES & Renewable Energy System \\
\hline RFC & Reason For Concern \\
\hline RPS & Renewable Portfolio Standard \\
\hline SAR & Second Assessment Report \\
\hline SM & Supplementary Material \\
\hline $\mathrm{SO}_{2}$ & Sulfur Dioxide \\
\hline SPM & Summary for Policymakers \\
\hline SRES & Special Report on Emissions Scenarios \\
\hline SREX & $\begin{array}{l}\text { Special Report on Managing the Risks of Extreme } \\
\text { Events and Disasters to Advance } \\
\text { Climate Change Adaptation }\end{array}$ \\
\hline SRM & Solar Radiation Management \\
\hline SRREN & $\begin{array}{l}\text { Special Report on Renewable Energy } \\
\text { Sources and Climate Change Mitigation }\end{array}$ \\
\hline SYR & Synthesis Report \\
\hline TCR & Transient Climate Response \\
\hline
\end{tabular}

TCRE Transient Climate Response to Cumulative $\mathrm{CO}_{2}$ Emissions

TFE Thematic Focus Element

TS Technical Summary

UHI Urban Heat Island

UNFCCC United Nations Framework

Convention on Climate Change

Watt

WG Working Group

WMGHG Well-Mixed Greenhouse Gas 

ANNEX W

Authors and Review Editors 


\section{Core Writing Team Members}

\section{ALLEN, Myles R.}

University of Oxford

UK

BARROS, Vicente R.

IPCC WGII Co-Chair

University of Buenos Aires

Argentina

BROOME, John

University of Oxford

UK

\section{CHRIST, Renate}

Secretary of the IPCC

IPCC Secretariat, World Meteorological Organization (WMO)

Switzerland

CHURCH, John A.

Commonwealth Scientific and Industrial

Research Organisation (CSIRO)

Australia

CLARKE, Leon

Pacific Northwest National Laboratory

USA

CRAMER, Wolfgang

IV Potsdam Institute for Climate Impact Research / Institut

Méditerranéen de Biodiversité et d'Ecologie marine et continentale (IMBE)

Germany/France

DASGUPTA, Purnamita

University of Delhi Enclave

India

DUBASH, Navroz

Centre for Policy Research, New Delhi

India

\section{EDENHOFER, Ottmar}

IPCC WGIII Co-Chair

Potsdam Institute for Climate Impact Research

Germany

ELGIZOULI, Ismail

IPCC Vice-Chair

Sudan

FIELD, Christopher B.

IPCC WGII Co-Chair

Carnegie Institution for Science

USA
FORSTER, Piers

University of Leeds

UK

\section{FRIEDLINGSTEIN, Pierre \\ University of Exeter \\ UK}

\section{FUGLESTVEDT, Jan}

Center for International Climate and

Environmental Research (CICERO)

Norway

GOMEZ-ECHEVERRI, Luis

International Institute for Applied Systems Analysis (IIASA)

Austria

HALLEGATTE, Stephane

World Bank

USA

HEGERL, Gabriele C.

University of Edinburgh

UK

\section{HOWDEN, Mark}

Commonwealth Scientific and Industrial

Research Organisation (CSIRO)

Australia

JIMÉNEZ CISNEROS, Blanca

Universidad Nacional Autónoma de México / United

Nations Educational, Scientific and Cultural Organization (UNESCO)

Mexico/France

KATTSOV, Vladimir

Voeikov Main Geophysical Observatory

Russian Federation

KEJUN, Jiang

Energy Research Institute

China

LEE, Hoesung

IPCC Vice-Chair

Keimyung University

Republic of Korea

MACH, Katharine J.

IPCC WGII Technical Support Unit

USA

MAROTZKE, Jochem

Max Planck Institute for Meteorology

Germany 
MASTRANDREA, Michael D.

IPCC WGII Technical Support Unit

USA

MEYER, Leo

IPCC Synthesis Report Technical Support Unit The Netherlands

MINX, Jan

IPCC WGIII Technical Support Unit

Germany

MULUGETTA, Yacob

University of Surrey

UK

O'BRIEN, Karen

University of Oslo

Norway

OPPENHEIMER, Michael

Princeton University

USA

PACHAURI, R. K.

IPCC Chair

The Energy and Resources Institute (TERI)

India

PEREIRA, Joy J.

Universiti Kebangsaan Malaysia

Malaysia

PICHS-MADRUGA, Ramón

IPCC WGIII Co-Chair

Centro de Investigaciones de la Economía Mundial

Cuba

PLATTNER, Gian-Kasper

IPCC WGI Technical Support Unit

Switzerland

PÖRTNER, Hans-Otto

Alfred-Wegener-Institute

Germany

POWER, Scott B.

Bureau of Meteorology

Australia

PRESTON, Benjamin

Oak Ridge National Laboratory

USA

QIN, Dahe

IPCC WGI Co-Chair

China Meteorological Administration

China
RAVINDRANATH, N. H.

Indian Institute of Science

India

REISINGER, Andy

NZ Agricultural Greenhouse Gas Research Centre

New Zealand

RIAHI, Keywan

International Institute for Applied Systems Analysis (IIASA)

Austria

RUSTICUCCI, Matilde

Universidad de Buenos Aires

Argentina

SCHOLES, Robert

Council for Scientific and Industrial Research (CSIR)

South Africa

SEYBOTH, Kristin

IPCC WGIII Technical Support Unit

USA

SOKONA, Youba

IPCC WGIII Co-Chair

South Centre

Switzerland

STAVINS, Robert

Harvard University

USA

STOCKER, Thomas F.

IPCC WGI Co-Chair

University of Bern

Switzerland

TSCHAKERT, Petra

Pennsylvania State University

USA

VAN VUUREN, Detlef

Netherlands Environmental Assessment Agency (PBL)

The Netherlands

VAN YPERSELE, Jean-Pascal

IPCC Vice-Chair

University of Louvain

Belgium 


\section{Extended Writing Team Members}

BLANCO, Gabriel

Universidad Nacional del Centro de la Provincia de Buenos Aires Argentina

\section{EBY, Michael}

University of Victoria

Canada

EDMONDS, Jae

University of Maryland

USA

FLEURBAEY, Marc

Princeton University

USA

\section{GERLAGH, Reyer}

Tilburg University

The Netherlands

KARTHA, Sivan

Stockholm Environment Institute

USA

KUNREUTHER, Howard

The Wharton School of the University of Pennsylvania USA

ROGELJ, Joeri
International Institute for Applied Systems Analysis (IIASA)

Austria

SCHAEFFER, Michiel

Wageningen University

Germany/The Netherlands

SEDLÁČEK, Jan

ETH Zurich

Switzerland

SIMS, Ralph

Massey University

New Zealand

ÜRGE-VORSATZ, Diana

Central European University

Hungary

VICTOR, David G.

University of California San Diego

USA
YOHE, Gary

Wesleyan University

USA

\section{Review Editors}

ALDUNCE, Paulina

University of Chile

Chile

CHEN, Wenying

Tsinghua University

China

DOWNING, Thomas

Global Climate Adaptation Partnership

UK

JOUSSAUME, Sylvie

Laboratoire des Sciences du Climat et de l'Environnement (LSCE)

Institut Pierre Simon Laplace

France

KUNDZEWICZ, Zbigniew

Polish Academy of Sciences

Poland

PALUTIKOF, Jean

Griffith University

Australia

SKEA Jim

Imperial College London

UK

TANAKA, Kanako

Japan Science and Technology Agency

Japan

TANGANG, Fredolin

National University of Malaysia

Malaysia

ZHANG, Xiao-Ye

China Meteorological Administration

China 


\section{ANNEX}

Expert Reviewers 


\section{AKIMOTO, Keigo}

Research Institute of Innovative Technology for the Earth Japan

\section{ALCAMO, Joseph}

University of Kassel

Germany

\section{ALEXANDER, Lisa V.}

University of New South Wales

Australia

\section{AMESZ, Bert}

The Netherlands

ARAKI, Makoto

Forestry and Forest Products Research Institute Japan

\section{ARROYO CURRÁS, Tabaré}

WWF International

Mexico

BINDOFF, Nathaniel L.

University of Tasmania

Australia

BORGES LANDÁEZ, Pedro Alfredo

Ministry of Science and Technology

Venezuela

\section{BRAGHIERE, Renato}

University of Reading

UK

\section{BRUNO, John}

The University of North Carolina at Chapel Hill USA

\section{CARTER, Peter}

Climate Emergency Institute

Canada

\section{CASEY, Michael}

Carbon Virgin

Ireland

CHOI, Young-June

Seoul Metropolitan Government

Republic of Korea

COHEN, Stewart

Environment Canada

Canada

\section{CONVERSI, Alessandra}

National Research Council of Italy

Italy

DING, Yihui

National Climate Center, Meteorological Administration

China

\section{DIXON, Tim}

International Energy Agency Greenhouse Gas R\&D Programme (IEAGHG)

UK

DONG, Wenjie

Bejing Normal University

China

EKHOLM, Tommi

Technical Research Centre of Finland (VTT)

Finland

ESASHI, Kei

The Federation of Electric Power Companies

Japan

FISCHLIN, Andreas

ETH Zurich

Switzerland

FITZSIMMONS, Jason

Chartered Institution of Building Services Engineers (CIBSE)

UK

\section{GALE, David}

Royal Institute of British Architects

UK

HABERL, Helmut

Alpen-Adria Universität Klagenfurt, Wien, Graz

Austria

HARNISCH, Jochen

KfW Bankengruppe

Germany

HOUSE, Joanna

Bristol University

UK

JU, Hui

Chinese Academy of Agricultural Science

China

KAINUMA, Mikiko

National Institute for Environmental Studies Japan 
KATBEH BADER, Nedal

Environment Quality Authority

Palestine

KAZUNO, Hirofumi

The Kansai Electric Power Co., Inc.

Japan

KHESHGI, Haroon

ExxonMobil Research and Engineering Company

USA

KOSONEN, Kaisa

Greenpeace

Finland

LEFFERTSTRA, Harold

Norwegian Environment Agency (retired)

Norway

\section{LIU, Qiyong}

National Institute for Communicable Disease Control and Prevention China

\section{LLASAT, Maria-Carmen}

University of Barcelona

Spain

LYNN, Jonathan

IPCC Secretariat, World Meteorological Organization (WMO)

Switzerland

\section{MA, Shiming}

Chinese Academy of Agricultural Sciences

China

MASUDA, Kooiti

Japan Agency for Marine-Earth Science and Technology Japan

MÉNDEZ, Carlos

Instituto Venezolano de Investigaciones Científicas

Venezuela

MENZEL, Lena

Alfred Wegener Institute

Germany

MOJTAHED, Vahid

Università Ca' Foscari di Venezia

Italy

\section{MOLINA, Tomas}

Universitat de Barcelona

Spain

\section{MURATA, Akihiko}

Research and Development Center for Global Change

Japan

NDIONE, Jacques Andre

Centre de Suivi Ecologique

Senegal

OZDEMIR, Eray

General Directorate of Forestry

Turkey

\section{PALTSEV, Sergey}

Massachusetts Institute of Technology

USA

\section{PLANTON, Serge}

Météo-France

France

PLATTNER, Gian-Kasper

IPCC WGI Technical Support Unit

Switzerland

\section{POLOCZANSKA, Elvira}

Commonwealth Scientific and Industrial Research Organisation (CSIRO)

Australia

PORTER, John

University of Copenhagen

Denmark

POWER, Scott B.

Bureau of Meteorology

Australia

RAHOLIJAO, Nirivololona

National Meteorological Office

Madagascar

RAMASWAMY, Venkatachalam

National Oceanic and Atmospheric Administration (NOAA)

USA

RHEIN, Monika

University of Bremen

Germany

ROGNER, Hans-Holger

Institute for Applied Systems Analysis (IIASA) (retired)

Austria

SCHEI, Tormod Andre

Statkraft AS

Norway 
SCHLEUSSNER, Carl-Friedrich

Potsdam Institute for Climate Impact Research

Germany

SHINE, Keith

University of Reading

UK

\section{SOUTHWELL, Carl}

Risk and Policy Institute

USA

STOTT, Peter A.

Met Office Hadley Centre

UK

\section{SU, Mingshan}

National Center for Climate Change Strategy and International Cooperation

China

SUAREZ RODRIGUEZ, Avelino G.

Institute of Ecology and Systematics

Cuba

SUGIYAMA, Taishi

The Central Research Institute of Electric Power Industry (CRIEPI) Japan

TAKAHASHI, Kiyoshi

National Institute for Environmental Studies

Japan

TAKASHI, Hongo

Mitsui Global Strategic Studies Institute

Japan

TAKEMURA, Toshihiko

Kyushu University

Japan

TATTERSHALL, David

USA

THORNE, Peter W.

Nansen Environmental and Remote Sensing Center (NERSC)

Norway

TOL, Richard

University of Sussex

UK

TSUTSUI, Junichi

The Central Research Institute of Electric Power Industry (CRIEPI) Japan

URGE-VORSATZ, Diana

Central European University

Hungary
WARD, Robert

London School of Economics (LSE)

UK

WARREN, Rachel

University of East Anglia

UK

WEIR, Tony

University of the South Pacific

Australia

WRATT, David

National Institute of Water and Atmospheric Research (NIWA)

New Zealand

WU, Jian Guo

Chinese Research Academy of Environmental Sciences

China

WUEBBLES, Donald

University of Illinois

USA

\section{XIA, Chaozong}

China

YAMIN, Farhana

University College London (UCL)

UK

YUTA, Sasaki

Tohoku Electric Power Co., Inc.

Japan

ZHANG, Chengyi

National Climate Center

China

ZHANG, Guobin

State Forestry Administration (SFA)

China

ZHAO, Zong-Ci

China Meteorological Administration (CMA)

China

ZHOU, Guomo

Zhejiang A\&F University

China

ZHU, Songli

Energy Research Institute

China 


\section{ANNEX $\mathbf{V}$}

Publications by the Intergovernmental Panel on Climate Change 


\section{Assessment Reports}

Fifth Assessment Report

Climate Change 2013: The Physical Science Basis

Contribution of Working Group I to the Fifth Assessment Report

Climate Change 2014: Impacts, Adaptation, and Vulnerability

Contribution of Working Group II to the Fifth Assessment Report

Climate Change 2014: Mitigation of Climate Change

Contribution of Working Group III to the Fifth Assessment Report

Climate Change 2014: Synthesis Report

A Report of the Intergovernmental Panel on Climate Change

Fourth Assessment Report

Climate Change 2007: The Physical Science Basis

Contribution of Working Group I to the Fourth Assessment Report

Climate Change 2007: Impacts, Adaptation and Vulnerability

Contribution of Working Group II to the Fourth Assessment Report

Climate Change 2007: Mitigation of Climate Change

Contribution of Working Group III to the Fourth Assessment Report

Climate Change 2007: Synthesis Report

A Report of the Intergovernmental Panel on Climate Change

Third Assessment Report

Climate Change 2001: The Scientific Basis

Contribution of Working Group I to the Third Assessment Report

Climate Change 2001: Impacts, Adaptation, and Vulnerability

Contribution of Working Group II to the Third Assessment Report

Climate Change 2001: Mitigation

Contribution of Working Group III to the Third Assessment Report

Climate Change 2001: Synthesis Report

Contribution of Working Groups I, II and III to the Third Assessment Report

Second Assessment Report

Climate Change 1995: Science of Climate Change

Contribution of Working Group I to the Second Assessment Report

Climate Change 1995: Scientific-Technical Analyses of Impacts, Adaptations and Mitigation of Climate Change

Contribution of Working Group II to the Second Assessment Report

Climate Change 1995: Economic and Social Dimensions of Climate Change

Contribution of Working Group III to the Second Assessment Report
Climate Change 1995: Synthesis of Scientific-Technical Information Relevant to Interpreting Article 2 of the UN Framework Convention on Climate Change

A Report of the Intergovernmental Panel on Climate Change

Supplementary Reports to the First Assessment Report Climate Change 1992: The Supplementary Report to the IPCC Scientific Assessment

Supplementary report of the IPCC Scientific Assessment Working Group I

Climate Change 1992: The Supplementary Report to the IPCC Impacts Assessment

Supplementary report of the IPCC Impacts Assessment Working Group II

Climate Change: The IPCC 1990 and 1992 Assessments

IPCC First Assessment Report Overview and Policymaker Summaries and 1992 IPCC Supplement

First Assessment Report

Climate Change: The Scientific Assessment

Report of the IPCC Scientific Assessment Working Group I, 1990

Climate Change: The IPCC Impacts Assessment

Report of the IPCC Impacts Assessment Working Group II, 1990

Climate Change: The IPCC Response Strategies

Report of the IPCC Response Strategies Working Group III, 1990

\section{Special Reports}

Managing the Risks of Extreme Events and Disasters to Advance Climate Change Adaptation (SREX) 2012

Renewable Energy Sources and Climate Change Mitigation (SRREN) 2011

Carbon Dioxide Capture and Storage 2005

Safeguarding the Ozone Layer and the Global Climate System: Issues Related to Hydrofluorocarbons and Perfluorocarbons (IPCC/TEAP joint report) 2005

Land Use, Land-Use Change, and Forestry 2000

Emissions Scenarios 2000

Methodological and Technological Issues in Technology Transfer 2000

Aviation and the Global Atmosphere 1999

The Regional Impacts of Climate Change: An Assessment of Vulnerability 1997 
Climate Change 1994: Radiative Forcing of Climate Change and an Evaluation of the IPCC IS92 Emission Scenarios 1994

\section{Methodology Reports and Technical Guidelines}

2013 Revised Supplementary Methods and Good Practice Guidance Arising from the Kyoto Protocol (KP Supplement) 2014

2013 Supplement to the 2006 IPCC Guidelines for National Greenhouse Gas Inventories: Wetlands (Wetlands Supplement) 2014

2006 IPCC Guidelines for National Greenhouse Gas Inventories (5 Volumes) 2006

Definitions and Methodological Options to Inventory Emissions from Direct Human-induced Degradation of Forests and Devegetation of Other Vegetation Types 2003

Good Practice Guidance for Land Use, Land-use Change and Forestry 2003

Good Practice Guidance and Uncertainty Management in National Greenhouse Gas Inventories 2000

Revised 1996 IPCC Guidelines for National Greenhouse Gas Inventories (3 volumes) 1996

IPCC Technical Guidelines for Assessing Climate Change Impacts and Adaptations 1994

IPCC Guidelines for National Greenhouse Gas Inventories (3 volumes) 1994

Preliminary Guidelines for Assessing Impacts of Climate Change 1992

\section{Technical Papers}

Climate Change and Water

IPCC Technical Paper VI, 2008

Climate Change and Biodiversity

IPCC Technical Paper V, 2002

Implications of Proposed $\mathrm{CO}_{2}$ Emissions Limitations

IPCC Technical Paper IV, 1997

Stabilization of Atmospheric Greenhouse Gases: Physical, Biological and Socio-Economic Implications

IPCC Technical Paper III, 1997

An Introduction to Simple Climate Models Used in the IPCC Second Assessment Report

IPCC Technical Paper II, 1997
Technologies, Policies and Measures for Mitigating Climate Change

IPCC Technical Paper I, 1996

For a list of Supporting Material published by the IPCC (workshop and meeting reports), please see www.ipcc.ch or contact the IPCC Secretariat, clo World Meteorological Organization, 7 bis Avenue de la Paix, Case Postale 2300, Ch-1211 Geneva 2, Switzerland 

Index 
Note: An asterisk $\left({ }^{*}\right)$ indicates the term also appears in the Glossary. Page numbers in bold indicate page spans for the four Topics. Page numbers in italics denote figures, tables and boxed material.

\section{A}

Abrupt climate change ${ }^{*}, 13,16,65,73-74$

Adaptation*, 17-31, 76-112

approaches, variety of, 27, 94, 95, 96

characteristics of, 19-20, 79-81

co-benefits, 17, 20, 26, 80-81, 90, 91, 98

cooperative action in, 17, 26, 29, 76, 94, 102, 105,

106

emissions reductions and, 17,76

enabling factors and constraints, 19-20, 26, 80,

$94,95,111$

equity and fairness in, 17, 76-77, 95

finance, 30-31, 97, 107, 110-111, 110-111

first step in, 19, 80

funding gap, 31, 111

future pathways, 17-26, 76-91

interactions with mitigation, 17-18, 20, 26, 76, 77,

$80-81,90,98,112$

maladaptation, $20,77,80$

near-term decisions, 77, 79

place- and context-specificity of, $79-80$

planning and implementation, 19-20, 26, 29-30,

$31,54,80,94,95-97,96,98,106,107,112$

policy approaches for, 26, 29-31, 94, 96, 102-111

risk management/reduction by, 14, 17-19, 18,

$65-67,65,70-71,76,77-79,79,94,108$

risks/side effects of, 17, 76, 91

risks compared with risks from climate change,

$17,19,77$

sustainable development and, 17, 19, 31, 76, 79, 95

transformation and, 20,27, 76, 80, 96

Adaptation deficit* ${ }^{*}, 91,95$

Adaptation experience, 26, 54, 106-107, 106

Adaptation limits*, 19-20, 72, 79

exceedance of, 20,67, 77, 80

Adaptation options, 26, 27, 76, 94, 95-98, 96 by sectors, 95-97, 98

Adaptation pathways, 17-26, 76-91

characteristics of, 19-20, 79-81

Adaptation potentials, $65,70-71$

Adaptive capacity* $26,77,80,94$

Aerosols, 44, 90

Afforestation* , 28, 29, 81, 102, 112

AFOLU (Agriculture, Forestry and Other Land

Use) ${ }^{*}, 28,30,101,104,108$

Agriculture, 16, 29, 69, 81, 98, 102

See also Crop yields

Antarctic ice sheet, 4, 16, 42, 74
Anthropogenic emissions, 3, 4-5, 5, 8, 16, 18, 20 , $44,45-47,45-47,54,63-64,73-74,74,78$

Anthropogenic forcings, 5, 6, 44-47, 45, 48, 48

Arctic region, rapid warming in, 4, 10, 60

Arctic sea ice, 4, 12, 48, 62

anthropogenic influences on, 5, 48, 49

observed changes, 4, 41, 42, 48, 49

projected changes, $12,62,74$

Atlantic Meridional Overturning Circulation (AMOC), 60-62, 74

Atmosphere, 2, 3, 40, 41, 42, 47, 58-60, 82

Attribution. See Detection and attribution

B

Biodiversity* $13,64,65,67,98,109,112$

Bioenergy, 25, 82, 85, 86, 102

Bioenergy and Carbon Capture and Storage

(BECCS) $^{*}, 22,23,24,28,81,82,85,89,100$

Biogeochemistry, 62

C

Cancún Pledges* ${ }^{*} 23,24,84,85$

Cap and trade, 30, 107

Carbon cycle*, 45, 56, 56, 62

Carbon dioxide $\left(\mathrm{CO}_{2}\right)$

$\mathrm{CO}_{2}$-equivalents ${ }^{*}, 5,20-23,21-24,28,45-46,46$,

$47,81,82-87,84-85,99-100,99,101$

emissions, drivers of, 4, 46-47, 47, 81

emissions, increase in, 3, 4-5, 5, 44, 44, 45-47,

45-47

emissions scenarios, 8, 18-19, 18, 20-24, 21-23,

$28,28,57,81-86,82-86,99,101$

emissions, warming and, 8-10, 9, 18-19, 18, 20,

$21,62-63,63-64,78$

projections, 8, 9, 16, 63-64, 73-74, 74

radiative forcing and, $43,44,45$

removal from atmosphere, 16, 62-63, 74

See also Emissions

Carbon Dioxide Removal (CDR) ${ }^{*}, 21,23,24,81$, 82,89

Carbon dioxide capture and storage (CCS) ${ }^{*}, 22$, $24,25,28,82,85,109,110$

Carbon price* , 24, 25, 30, 106, 107, 108, 109

Carbon sequestration, 31, 101, 112

Carbon sinks* , 20, 28, 45, 67, 81, 98

Cascading impacts, 51,52

Causes. See Detection and attribution

Certainty, 2, 37

Clean Development Mechanism (CDM), 105-106 108

Climate change* , 2-16, 40-74

adaptation and mitigation and , 17-31, 76-112

attribution of, 47-51

beyond 2100, 16, 73-74 causes of, 4-5, 44-51

comprehensive strategies for, 91

decision making about, 17, 76-77, 107

drivers of, 4, 5-96, 8-10, 9, 44-47, 47, 56-58, 62,

$70-71,81,84$

emissions reductions, effects on, 17-19, 18, 20,

$56,84-85$

future changes, 8-16, 56-74

future risks and impacts, 13-16, 17-19, 18, 77-79, 78

impacts attributed to, 6, 7, 49-51, 50-52

irreversible or abrupt changes, $13,16,65,73-74$

limiting, 8, 17, 20, 56, 65, 84-85

risk amplification by, 13, 16, 64, 66, 77, 78

timescales, 13, 16, 62-63, 63, 73-74, 77

Climate extreme. See Extreme weather events

Climate finance* $, 95,109-110,111$

Climate models*, 12, 43, 56-58, 56, 58

confidence and uncertainty in, 56

Climate-resilient pathways* ${ }^{*}$ 17, 31, 76, 77, 90

Climate sensitivity* $, 48,49,62$

Climate system*

drivers of changes in, 4-5, 8-10, 44-47, 56-58, 81, 84

human influence on, 2, 4-5, 5, 8, 9, 44, 48-49, 51, 63-64

observed changes in, 2-4, 3, 12, 40-44, 41-43, 49-51, 50-52

projected changes in, $10-13,16,56,58-64,59-61$, 63-64

responses of, 62-63

timescales of change, $62-63,63$

warming of, 2-4, 3, 62-63

$\mathrm{CO}_{2}$. See Carbon dioxide

Coastal systems, $13,15,66,67,97,98$

Co-benefits* $17,20,26,30,77,78-79,80-81,90$, 90-91, 98, 102, 103-104, 107, 109

Confidence* $, 2,37,56$

Cooperation, 17, 26, 29, 76, 89, 94, 102, 105, 106

Coral reefs, 13, 67, 68, 72, 74, 97

Cost-effectiveness * , 24, 24-25, 28-30, 77, 84-86, $85-86,98,99,102,107,112$

Costs

of mitigation, 17, 24-25, 24-25, 28-30, 84-86,

$85-86,98,99,102$

of mitigation delays, $19,24,25,79,85,86$

See also Climate finance

Crop yields, 13, 15, 51, 69, 69, 98

Cryosphere, 2, 42, 47, 52, 62

D

Decarbonization *, 5, 78, 81, 98, 99-100

Decision making, 17, 19, 29, 76-77, 107

Deforestation* ${ }^{*} 28,29,67,83,102$

Delay in mitigation, effects of, $17,19,20,24,25$, $31,76,77,79,81,84-85,86,90$ 
Detection and attribution * 4-8, 7, 45-51, 50-51 See also Humans

Disaster risk management, 26, 27, 31, 54, 91, 94, $95,96,97,106,111$

Droughts* $8,15,36,51,53,69,97,98$

\section{E}

Early warning systems ${ }^{*}, 27,95,96,97$

Economic diversification, 19, 27, 30, 31, 80, 96

Economic growth and development, 64, 94 emissions and, 4, 8, 20, 44, 46-47, 47, 56, 81

Economic indicators, aggregate, 78

Economic instruments, 30, 107-109, 108

Economic losses, 53, 73

Ecosystem services* ${ }^{*}$ 13, 20, 64, 65, 67, 81

Ecosystems* ${ }^{*}$ 8, 13, 16, 20, 26, 27, 53, 64, 67, 74, 97 key risks, $65,65,66,67,74$

management, 27, 29, 96, 97

El Niño Southern Oscillation (ENSO)* $4,40,56$, 60

Emissions

anthropogenic, 3, 4-5, 5, 8, 16, 18, 20, 44, 45-47, $45-47,54,63-64,73-74,74,78$

$\mathrm{CO}_{2}$-equivalent ${ }^{*}, 5,20-23,21-24,28,45-46,46$,

$47,81,82-87,84-85,99-100,99,101$

as driver of climate change, $4-5,8-10,9,18,19$,

$44,45-47,45,56-58,62,84$

drivers of, $4,8,20,44-47,47,56,81$

economic assessment and, 30, 79, 85, 86

future risks and, 8-16, 17-19, 18, 77-79, 78

metrics for, $23,87-88$

of non- $\mathrm{CO}_{2}$ gases, $23,28,84,87,99$

observed changes, 2, 3, 4-5, 5, 44, 44, 45-47,

45-47, 54

projections( See Emissions scenarios)

reductions, $8,17-19,18,20-24,28,30,56,76,86$,

98-100, 99-101

reductions, challenges of, 20, 81

reductions, substantial, 8-10, 17-19, 18, 19, 20,

$24,28,56,63,77-78,81,110$

relationship with climate changes, 3, 4, 17, 18, 86

by specific gases, 5,46

temperature (warming) and, 8-10, 9, 18-19, 18,

20-24, 56, 58, 62-63, 81-86, 83

Emissions scenarios* ${ }^{*} 8,18-19,18,20-24,21-24$, $28,28,60-61,63-64,74,81-86,82-86$

baseline ${ }^{*}, 8,20,21,22,24,24,28,28,82,85,99$, 110

climate change risks and, $8,18-19,18,73-74$

mitigation pathways and, 18, 20-23, 21-23, 78,

81-86, 98-100, 99-101

overshoot scenarios ${ }^{*}, 20-23,22,81,83,89$

overview of, $21-23,83,83$

RCPs, 8, 9, 10, 11, 16, 21, 22, 56-62, 57, 59-61,

$63-64,74,74$

risk and, 66 sea-level rise and, 16

specific sectors and gases, 28, 46, 47, 99, 99

SRES scenarios, 57, 58

standard set of, 56-58, 57

temperature and, 8-10, 9, 16, 18-19, 18, 20-24,

$22,62-63,81$

Energy access* ${ }^{*}, 30,109$

Energy accumulation in climate system, 4, 42

Energy demand, 29, 99-100

Energy efficiency, 30, 81, 110

Energy intensity, 47, 47, 94, 98-99

Energy price. See Carbon price

Energy production, 28, 28, 30, 31, 81, 99-100, 100-101, 103, 110

decarbonizing of, $28,98,99-100$

low-carbon energy, $21,23,28,30,82,84,85,94$, $100,100,110$

policy instruments, 108

Equity, 17, 76-77, 89, 90, 95, 109

Exposure $^{*}, 8,13,16,20,36,53,54,58,64,76,96$ reduction of, 19, 27, 80

Extinction risk, 13, 19, 51, 65, 67

Extreme weather events* $, 7-8,53$

economic losses from, 53

human influences, 8,53

observed changes, 7-8, 53

precipitation, $7,8,10,11,15,53,58,60$

projections, 10, 11, 58

as Reason for Concern, 18, 18, 72-73, 78

risks due to, 19,65

sea level, 7, 8, 53

temperature, 7-8, 10, 53, 60

$\mathbf{F}$

Finance, 29, 30-31, 95, 95, 97, 102, 107, 109-110, 110-111

funding gap, 31, 111

Fisheries, 13, 15, 67, 68, 97

Floods*, 8, 15, 53, 67

Food production, 15, 16, 67, 68-69, 69, 97

Food security ${ }^{*}, 13,16,19,64,65,69,109$

Forests ${ }^{*}, 29,52,67,81,102$

afforestation* ${ }^{*} 28,29,81,82-83,102$

deforestation* ${ }^{*} 28,29,67,83,102$

Future changes, risks, and impacts, 8-16, 56-74 See also Projected changes

Future pathways, 17-26, 76-91

adaptation pathways, 19-20, 79-81

decision making and, 17, 19, 76-77, 107

mitigation pathways, 20-26, 81-86

G

Geoengineering * 89

Glaciers, 5, 48, 56 observed changes, 5, 42, 48

projected changes, 12,62

Global aggregate impacts, $18,18,72-73,73,78$

Global Temperature change Potential (GTP) ${ }^{*}$, 87-88

Global Warming Potential (GWP)* $87-88$

Governments/governance, 17, 26, 29-30, 31, 89, 112

adaptation and, 19, 26, 54, 80, 94, 95, 106, 107 See also Policies

Greenhouse gas emissions. See Emissions

Greenland ice sheet, 5, 48

observed changes, 4, 5, 42, 48

projected changes, 16,74

H

Heat waves* $7-8,10,53,58,60,69$

Human health, 13, 15, 31, 51, 65, 69, 97, 109

Human security, 16, 54, 64, 77, 97

Humans

anthropogenic forcings, 5, 6, 44-47, 45, 48, 48 anthropogenic greenhouse gas emissions, 3, 4-5, $5,8,9,16,18,20,44,45-47,45-47,54,63-64$, $73-74,74,78$

human activities, constraints on, 15, 19, 65, 69, 77 influence on climate system, 2, 4-5, 5, 8, 9, 44, 48-49, 51, 63-64 responses to climate change (See adaptation; mitigation)

I

Ice sheets, 56

observed losses, 4, 5, 42, 48

projected losses, 16,74

Impacts*, 8-16, 56-74

on all continents and oceans, v, 6, 47, 49

attribution of, 47-51, 50-52

cascading, 51,52

of climate change, $2,6,7,13-16,49-51,50-52$, 64-73

distribution of, $18,18,72-73,78$

exposure and vulnerability and, 58, 58

of extreme events, 53

future, 8-16, 56-74

global aggregate, 18, 18, 72-73, 73, 78

high, severe, widespread, and irreversible, 8, 13,

$17,18-19,56,62-63,64,65,77,79$

models of, 58,58

Reason for Concern and, 18, 18, 72-73

risk reduction for, 65,65

timescales of, 13, 16, 62-63, 77

See also Observed changes

Indigenous peoples, 19, 26, 27, 80, 95

Information measures, 30, 95, 108, 109 
Infrastructure, 15, 26, 29, 69, 79, 94, 95

Institutions, 26, 27, 29-30, 94, 95, 96, 105, 107

Integrated responses, 26, 28, 31, 54, 94, 98, 112

International cooperation, 17, 29, 76, 102, 105,

106

Investments, 26, 30-31, 94, 108, 109, 110-111, 110-111

Irreversible impacts, 8, 13, 17, 18-19, 56, 62-63, $64,77,79$

Irreversible or abrupt changes* ${ }^{*} 13,16,65,73-74$

K

Kyoto Protocol, 29, 84, 105-106

$\mathbf{L}$

Land use and land-use change * $27,31,56,96$ AFOLU, 28, 30, 101, 104, 108

RCPs and, 57

Large-scale singular events, 18, 18, 72-73, 78, 79

Likelihood*. See Confidence

Livelihoods, 26, 27, 64, 65, 67, 90, 94, 96, 97

Local governments, 19, 29, 80, 106, 107

Low-carbon energy supply, 21, 23, 28, 30, 82, 84 , $85,94,100,100,110$

M

Methane, 4, 44, 44, 57, 84

Migration

of human populations, 16,73

of species (See range shifts)

Mitigation*, 17-31, 76-112

behaviour, lifestyle, and culture and, 26, 27, 29 ,

$81,94,95-96,98-102$

characteristics of, 20-26, 81-86

co-benefits of, 17, 20, 30, 77, 78-79, 80-81, 90,

$90-91,98,102,103-104,107,109$

cooperative action in, 17, 26, 29, 76, 94, 102, 105

delay, effects of, $17,19,20,24,25,31,76,77,79$,

$81,84-85,86,90$

emissions increases despite, 54

emissions reductions and, 17, 76, 81-86, 98-100 99-101

enabling factors and constraints, 26, 94, 95, 111

equity and fairness in, 17, 76-77, 109

future pathways, 17-26, 76-91

influence on climate change, 86

integrated approach, 26, 28, 31, 54, 94, 98, 112

interactions with adaptation, 17-18, 20, 26, 76,

$77,80-81,90,98,112$

national and sub-national, 106-109

near-term decisions, 17-18, 19, 77, 79

policy approaches for, 29-31, 102-111 risk reduction by, $14,17-19,18,76,77-79$

risks/side effects of, 17, 19, 30, 76, 78-79, 91, 102,

103-104, 107, 109

risks compared with risks from climate change,

$17,19,77,78-79$

warming levels without additional mitigation, 17 ,

$18-19,18,77,81$

Mitigation costs, 17, 24-25, 24-25, 28-30, 84-86,

$85-86,98,99,102$

cost-effectiveness, 24, 24-25, 28-30, 84-86, 85-86,

$98,99,102,107$

delays and, $19,24,25,79,85,86$

distribution of, 86

economic assessments, 79, 85, 86, 111

Mitigation options, 26, 28-29, 31, 90, 98-102, 99-101

by sectors, 28, 98-99, 99, 101

Mitigation pathways, 17-26, 76-91, 98-100, 99-101

characteristics of, $20-26,81-86$

emission metrics and, 23, 87-88

Mitigation scenarios*, 18-19, 18, 20-25, 21-24, 28, $28,30,81-86,82-86,98-100,99-101,110$

Models. See Climate models

N

National governments, 19, 29, 30, 80, 106-109

0

Observed changes, 2-8, 40-54

in climate system, 2-4, 3, 12, 40-44, 41-43, 47 49-51, 50-52

in emissions, 2, 3, 4-5, 5, 44, 44, 44-48, 45-47, 45- 47,54

extreme events, 7-8, 53

human influence and, 2, 5

impacts of, 6, 7, 49-51, 50-52

in temperature, 2-4, 3, 5, 7-8, 12, 40, 41, 43, 47, 49,61

Ocean, 40-41, 60-62, 97

cascading impacts in, 52

energy accumulation in, 4,42

heat content, 5, 45, 48, 49

modeling, 56

observed changes, 2, 3, 4, 5, 40-41, 41, 42

oxygen content, 13, 41, 51, 62

projected changes, 10, 11, 16, 60-62, 67

salinity of, $4,40,48$

thermal expansion, 42, 48, 56

warming of, $2,3,4,5,10,11,40,41,45,47-48$,

$49,58,60,67$

Ocean acidification*

impacts of, 51, 67, 74

observed increase, 4, 40-41, 45, 48 projections, 10, 12, 16, 58, 59, 62, 66, 74

risks associated, 13, 65, 66, 67

timescale of, 16,74

Overshoot scenarios* ${ }^{*}, 20-23,22,81,83,89$

P

Permafrost* ${ }^{*} 4,12,16,42,62,74$

Policies, 17, 29-31, 91, 94, 102-111

for adaptation, mitigation, technology, and

finance, 26, 29-31, 81, 94, 95, 96, 102-111

assessing, 76

decision making and, 17, 19, 29, 76-77, 107

emission metrics and, 87-88

sectoral instruments, 30, 107, 108

sustainable development and, 90, 91

Population growth, 4, 8, 20, 44, 46-47, 47, 56, 81

Poverty* ${ }^{*} 16,17,27,31,54,73,76,90,96$

Precipitation

extreme events, $7,8,10,11,15,53,58,60$

observed changes, $4,8,12,40,41,48,51,53,61$

projected changes, $11,12,60,61$

Private sector, 19, 29, 30, 80, 95, 97, 106, 107, 111

Projected changes, 10-13, 11, 56-74

basis for (models), 56,58

in climate system, 10-13, 16, 56, 58-64, 59-61,

63-64

confidence and uncertainty in, 56

ecosystems and services, 66,67

emissions scenarios and, 8, 9, 18-19, 18, 20-24,

$21-24,28,28,56,57,60-61,63-64,74,81-86$,

82-86

relative to $1986-2005,10,58$

See also Temperature projections

$\mathbf{R}$

Radiative forcing ${ }^{*}, 5,6,43,44,45,48,48$

Range shifts of species, $6,13,51,67$

Reasons for Concern*, 18, 18, 72-73, 77-78, 78

Regions

adaptation experience, 106, 106

impacts, 7, 50-51

irreversible changes, 16

key risks, 13, 14, 65, 65

mitigation initiatives, 106

temperature data, 49

Renewable energy, 22, 28, 30, 110

Representative Concentration Pathways (RCPs) * $8,9,10,11,16,21,22,56-62,59-61,63-64,74$, 74

description of, 57

Resilience*, 31, 94

climate-resilient pathways* ${ }^{*}$ 17, 31, 76, 77, 90 
Risk* , 8-16, 36, 56-74

of adaptation, 17, 76, 91

causes of, 58,64

from climate change, 13-16, 17-19, 18, 31, 36,

$64-73,66,76-79,78$

estimating, 58

future, 8-16, 56-74

of geoengineering, 89

with high temperatures, $15,16,18,66,73-74,77$, 78

key risks, $14,64-65,65,70-73$

of mitigation, 17, 19, 30, 76, 78-79, 91, 102,

103-104, 107, 109

models of, 58,58

new risks, due to climate change, 13,64

perception of, 17, 19, 77

quantification of, $36,58,79$

Reason for Concern and, 18, 18, 72-73, 77-78, 78

region-specific, 13, 14, 65

unavoidable, 19

uneven distribution of, 13, 64

Risk management/reduction* ${ }^{*}, 13,14,17-19$,

36,65

adaptation and mitigation and, 14, 17-19, 17-25,

18, 26, 65-67, 65, 70-71, 76, 77-79, 79, 94, 108

substantial emissions reductions, 19, 20, 77-78,

81

See also Disaster risk management

Rural areas, 16, 65, 69, 97

S

Scenarios, 17-26, 56-58, 81-86, 82-86

emissions, 8, 9, 18-19, 18, 20-24, 21-24, 28, 28,

60-61, 63-64, 74, 81-86, 82-86

overshoot* ${ }^{*}, 20-23,22,81,83,89$

RCPs, 8, 9, 10, 11, 16, 21, 22, 56-62, 57, 59-61,

$63-64,74,74$

SRES, 57,58

See also Emissions scenarios

Sea ice

anthropogenic influences on, 5, 48, 49

Arctic, 4, 5, 12, 41, 42, 48, 49, 62, 74

observed changes, 4, 5, 41, 42, 48, 49

projected changes, $12,59,62$

Sea level

extremes, 7, 8, 53

observed changes, 2, 3, 42-44, 61

thermal expansion and, $42,48,56$

Sea level rise

anthropogenic influences on, 5, 48

contributions to, 42, 44, 74

observed, 2, 3, 4, 5, 41, 42-44, 48

projected, $10,11,13,16,58,59-61,62,74,74$

risks associated with, $65,65,66,67,74$

timescale of, 16,74

variability in, 13,62
Seasonal activities, 6, 51

Sectors, 97, 98

adaptation options, 95-97, 98

GHG emissions by, 28, 46, 47, 88, 99, 99, 101

key risks, 65, 70-71

mitigation options, 28, 98-99, 99, 101

policy instruments, 30, 107, 108

Snow cover, 2, 4, 42, 47, 48, 51, 62

Solar irradiance, 10, 44, 58

Solar radiation management (SRM) ${ }^{*}, 25-26,89$

Species extinctions. See Extinction risk

Species range shifts. See Range shifts

SRES scenarios* ${ }^{*}, 57,58$

Subsidies, 30, 107-109, 108

Sustainable development*, 17, 31, 76-77

adaptation and mitigation and, 17, 19, 31, 76, 79

climate change as threat to, 31, 90

climate policy and, 31, 76, 90, 91

equity and, 17, 76-77, 109

future pathways, 17-26, 76-91

trade-offs, synergies and interactions, 31, 80-81, 90,112

transformations and, 20, 80

Synergies, 19, 20, 26, 31, 80-81, 90, 109, 112

$\mathbf{T}$

Technology, 20, 23, 24, 25, 26, 81, 85, 94, 95, 95 , 100

policies and, 29, 30, 102, 109

\section{Temperature}

emissions and, 8-10, 9, 16, 18-19, 18, 20-24, 22,

$56,58,62-63,63-64,78,83$

extremes, 7-8, 10, 53, 60

global mean surface temperature, $9,10,20$,

58-60, 59-61

Global Temperature change Potential (GTP), 87-88

human influence on, 4, 5, 8, 9, 44, 47-48, 48, 63,

63-64

mortality associated with, 8, 51, 53

observed changes, 2-4, 3, 5, 7-8, 12, 40, 41, 43,

49,61

observed changes, contributions to, 48,48

observed regional changes, 49

recent trends, 43, 48

risks from high temperatures, $15,16,18,66$,

73-74, 77, 78

timescale of changes, 62-63, 73-74

variability in, 2-4, 3, 40, 41, 43

See also Warming

Temperature projections, 8-10, 9, 11-12, 16, 20-24,

$56,58-60,59-61,63-64,73-74,74,83$

in discontinuance of SRM, 26

global mean peak surface temperature change, 62 mitigation and, 20-25, 21-23, 81

warming to $2^{\circ} \mathrm{C}$ above pre-industrial, 8-10, 11,19 ,

$20,22,23-24,60,60,62,63,74,77,81-82,83,85$ warming to above $2^{\circ} \mathrm{C}$ above pre-industrial, 10 , $11,19,20-21,22,24,60,60,74,77,81-82,83$

See also Emissions scenarios

Thermal expansion* $, 42,48,56$

Timescales of climate change and impacts, 13 , $62-63,73-74,77$

Trade-offs, 20, 26, 31, 80-81, 90, 95, 98, 98, 112

Transformation* ${ }^{*} 20,27,76,80,96$

U

Uncertainty* $17,20,36,37,56$

See also Confidence

UNFCCC (United Nations Framework Convention on Climate Change), 2, 18, 29, 36, 102, 105

Unique and threatened systems, $18,18,19,65$, $72-73,78$

Urban areas, 15, 31, 69, 97, 112

V

Values and valuation, 17, 19, 23, 36, 76-77, 96

Violent conflicts, $16,54,77$

Volcanic aerosols, 10, 43, 44, 56

Vulnerability ${ }^{*}, 8,13,26,36,53,54,94,96$

estimating and models, 58

reduction of, $19,27,80$

risks and, 58, 58, 64, 76

W

Warming

of climate system, 2-4, 3, 8, 9, 40-44, 43, 47, 48, 49, 62-63

$\mathrm{CO}_{2}$ emissions and, 3, 8-10, 9, 18-19, 18, 20-24,

$21,56,62-63,63,64,78$

feedbacks and, 62

human contribution to, 4, 5, 8, 9, 44, 47-48, 48,

63, 63-64

irreversibility of, 62-63

of ocean, $2,3,4,5,10,11,40,41,45,47-48,49$, $58,60,65,67$

projections of, $9,10,11,12,16,20-21,22,56$,

58-60, 59-61, 63, 74

risks in high warming scenarios, 66, 73-74, 77, 78

timescales of, 16, 20, 62-63, 73-74

without additional mitigation, 17, 18-19, 18, 77, 81

See also Temperature

Water

management, $27,31,96,97,98$

resources and quality, 13, 16, 20, 51, 69, 97, 98

security, 13, 67-69

Water cycle, 4, 5, 47, 48, 60 
$\mathbf{T}$

he Intergovernmental Panel on Climate Change (IPCC) is the leading international body for the assessment of climate change. It was established by the United Nations Environment Programme (UNEP) and the World Meteorological Organization (WMO) to provide an authoritative international assessment of the scientific aspects of climate change, based on the most recent scientific, technical and socio-economic information published worldwide. The IPCC's periodic assessments of the causes, impacts and possible response strategies to climate change are the most comprehensive and up-to-date reports available on the subject, and form the standard reference for all concerned with climate change in academia, government and industry worldwide. This Synthesis Report is the fourth element of the IPCC Fifth Assessment Report, Climate Change 2013/2014. More than 800 international experts assessed climate change in this Fifth Assessment Report. The three Working Group contributions are available from the Cambridge University Press:

Climate Change 2013 - The Physical Science Basis

Contribution of Working Group I to the Fifth Assessment Report of the IPCC

(ISBN 9781107661820 paperback; ISBN 9781107057999 hardback)

Climate Change 2014 - Impacts, Adaptation, and Vulnerability

Contribution of Working Group II to the Fifth Assessment Report of the IPCC

(Part A: ISBN 9781107641655 paperback; ISBN 9781107058071 hardback)

(Part B: ISBN 9781107683860 paperback; ISBN 9781107058163 hardback)

Climate Change 2014 - Mitigation of Climate Change

Contribution of Working Group III to the Fifth Assessment Report of the IPCC

(ISBN 9781107654815 paperback; ISBN 9781107058217 hardback)

Climate Change 2014 - Synthesis Report is based on the assessments carried out by the three Working Groups of the IPCC and written by a dedicated Core Writing Team of authors. It provides an integrated assessment of climate change and addresses the following topics:

- Observed changes and their causes

- Future climate changes, risks and impacts

- Future pathways for adaptation, mitigation and sustainable development

- Adaptation and mitigation 